\title{
Proximal Bodies in Hypersonic Flow
}

\author{
Thesis by \\ Stuart J. Laurence \\ In Partial Fulfillment of the Requirements \\ for the Degree of \\ Doctor of Philosophy
}

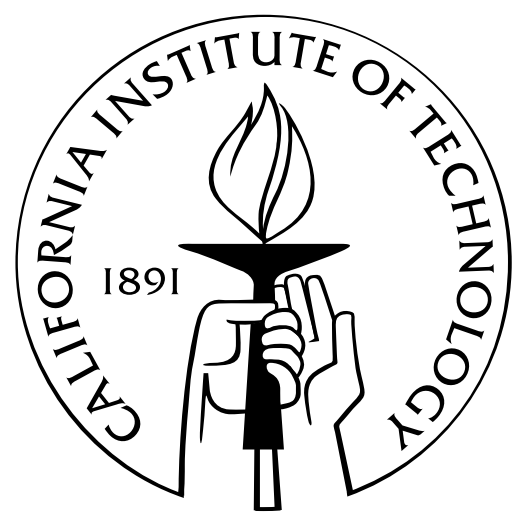

California Institute of Technology

Pasadena, California

2006

(Submitted April 25, 2006) 
(C) 2006

Stuart J. Laurence

All Rights Reserved 


\section{Abstract}

The problem of proximal bodies in hypersonic flow is encountered in several important situations, both natural and man-made. The present work seeks to investigate one aspect of this problem by exploring the forces experienced by a secondary body when some part of it is within the shocked region created by a primary body travelling at hypersonic speeds.

An analytical methodology based on the blast wave analogy is developed and used to predict the secondary force coefficients for simple geometries in both two and three dimensions. When the secondary body is entirely inside the primary shocked region, the nature of the lateral coefficient is found to depend strongly on the relative size of the two bodies. For two spheres, the methodology predicts that the secondary body will experience an exclusively attractive lateral force if the secondary diameter is larger then one-sixth the primary diameter. The analytical results are compared with

numerical simulations carried out using the AMROC software and good agreement is obtained if an appropriate normalization for the lateral displacement is used.

Results from a series of experiments in the T5 hypervelocity shock tunnel are also presented and compared with perfect-gas numerical simulations, again with good agreement. In order to model this situation experimentally, a new force-measurement technique for short-duration hypersonic facilities has been developed, and results from the validation experiments are included.

Finally, the analytical methodology is used to model two physical situations. First, the entry of a binary asteroid system into the Earth's atmosphere is simulated. Sec- 
iv

ond, a model for a fragmenting meteoroid in a planetary atmosphere is developed, and simulations are carried out to determine whether the secondary scatter patterns in the Sikhote-Alin crater field may be attributed to aerodynamic interactions between fragments rather than to secondary fragmentation. It is found that while aerodynamic interactions lead to increased secondary crater grouping, these groups do not exhibit the typically elliptical shape that we would expect secondary fragmentation to produce. 


\section{Acknowledgements}

This thesis would not have been possible without the input and assistance of a number of people. My advisor, Professor Hans Hornung, gave me the freedom to pursue my own ideas, but was ready with the right piece of advice when needed. I also thank the other members of my thesis committee, Professors Tim Colonius, Dale Pullin, Joseph Shepherd, and David Stevenson, for being willing to take the time to critique this work. Additional thanks to Professor Shepherd and also to Professor Ravichandran, for being willing to lend me their expensive (and in some cases, fragile) equipment.

The numerical simulations in this thesis would have been quite impossible without the diligence and patience of Dr. Ralf Deiterding; Drs. Joseph Olejniczak and James Quirk also provided much-needed assistance in this area. Thanks also to Professor Dan Meiron for providing access to the various computational facilities on which the majority of the simulations were run. The models used in the experimental investigation were skilfully and efficiently constructed by Mr. Ali Kiani in the Aero machine shop. A number of students and ex-students also provided assistance and input: in particular, Drs. Florian Pintgen, Daniel Lieberman, and Patrick Lemieux and Mr. Christopher Mouton. Thanks are also due to Mr. Mike Rubel for sorting out the various computer problems I encountered during my time in GALCIT.

I am especially grateful to our tunnel engineer, Mr. Bahram Valiferdowsi, whose technical skills are matched only by his irrepressible good spirit. I would also like to thank my professors at the University of Auckland, especially Professor Gordon Mallinson, for equipping me with the tools I needed to survive in an academic envi- 
ronment such as the one GALCIT provides. Thanks also to my family, especially my parents, Phil and $\mathrm{Su}$, for their continuous support.

Finally, I'd like to thank Dr. and Mrs. Gordon and Betty Moore, whose generous fellowship allowed me to live comfortably for the greater part of my graduate career at Caltech. 


\section{Contents}

Abstract

Acknowledgements $\quad$ V

Contents vii

List of Figures $\quad$ xi

List of Tables $\quad$ Xv

1 Introduction 1

1.1 Problem Definition and Scope of Current Work . . . . . . . . . . 1

1.2 Review of Previous Work . . . . . . . . . . . . . . 5

1.3 Discussion of Hypersonic Force-Measurement Techniques . . . . . . 6

2 Application of the Blast Wave Analogy to Proximal Hypersonic Bod$\begin{array}{ll}\text { ies } & 10\end{array}$

2.1 Introduction . . . . . . . . . . . . . . . . . . . 10

2.2 The Blast Wave Analogy . . . . . . . . . . . . . . . . . . . . . . . . 10

2.3 Modeling in Two Dimensions . . . . . . . . . . . . . . . . . . . 12

2.3.1 The Planar Blast Wave Analogy . . . . . . . . . . . . . . . . . 12

2.3.2 Proximal Bodies in Two Dimensions . . . . . . . . . . . . 16

2.3.2.1 Pressure Distribution on the Secondary Body . . . . 16

2.3.2.2 Drag and Lift Coefficients . . . . . . . . . 21 
viii

2.4 Modeling in Three Dimensions . . . . . . . . . . . . . . . . . . 27

2.4.1 The Axisymmetric Blast Wave Analogy . . . . . . . . . . . . . 27

2.4.2 Proximal Bodies in Three Dimensions . . . . . . . . . . . . 30

2.4.2.1 Pressure Distribution on the Secondary Body . . . . 30

2.4.2.2 Drag and Lift Coefficients . . . . . . . . . . . 34

3 Computational Modeling $\quad 40$

3.1 Introduction . . . . . . . . . . . . . . . . . 40

3.2 Two-Dimensional Computations . . . . . . . . . . . . . . . . . 40

3.2.1 Introduction . . . . . . . . . . . . . . . . . . . 40

3.2.2 Details of Computations . . . . . . . . . . . . . . . 41

3.2.3 Results.......................... 43

3.3 Three-dimensional Computations .............. 51

3.3 .1 Introduction . . . . . . . . . . . . . . . . 51

3.3.2 Details of Computations . . . . . . . . . . . . . 51

3.3.3 Results........................... 52

3.3.4 Refinement Study . . . . . . . . . . . . . . . . . 57

4 Comparison of Theoretical and Computational Results 61

4.1 Two-Dimensional Comparison . . . . . . . . . . . . . . 61

4.2 Three-Dimensional Comparison ................ 64

4.3 Discussion . . . . . . . . . . . . . . . . . . . 75

$\begin{array}{lll}5 & \text { Experimental Investigation } & 77\end{array}$

5.1 Introduction . . . . . . . . . . . . . . . . . 77

5.2 Experimental Procedure . . . . . . . . . . . . . . . . 77

5.2.1 The T5 Hypervelocity Shock Tunnel Facility . . . . . . . . . . 77

5.2.2 Experimental Setup . . . . . . . . . . . . . 80 
ix

5.2.3 Accelerometer Measurements ............. . 83

5.2.4 High-speed Camera Measurements . . . . . . . . . . . . . 85

5.3 Results . . . . . . . . . . . . . . . . . . . . . . 88

5.3.1 Technique Validation . . . . . . . . . . . . . . . 88

5.3.2 Proximal Bodies Experiments . . . . . . . . . . . . . . 94

5.4 Error Analysis . . . . . . . . . . . . . . . . . . . . . 101

5.4.1 Model Movement . . . . . . . . . . . . . . . . . . . . 101

5.4.2 Flow Unsteadiness . . . . . . . . . . . . . . . 103

5.4.3 Effect of the Model Support Components . . . . . . . . . . . . 105

5.4 .4 Flow Chemistry . . . . . . . . . . . . . . 106

5.4.5 Shot-Induced Structural Vibrations . . . . . . . . . . . . . 108

5.4.6 Misalignment of the Model . . . . . . . . . . . . . . . . . . 110

5.5 Movement of the Primary Sphere . . . . . . . . . . . . . . . . 111

6 Binary Asteroids in a Planetary Atmosphere 113

6.1 Introduction . . . . . . . . . . . . . . . . . . 113

6.2 Domain of Validity of the Blast Wave Methodology . . . . . . . . . . 114

6.3 Extension of Blast Wave Methodology . . . . . . . . . . . . . . . . . 120

6.3.1 Lift Coefficients . . . . . . . . . . . . . . . . . . 120

6.3.2 Drag Coefficients . . . . . . . . . . . . . 125

6.4 Simulating the Planetary Entry of a Binary Asteroid System . . . . . 129

6.4.1 Simulations of the Asteroid 2000 DP107 . . . . . . . . . . 129

6.4.1.1 Simulation Parameters . . . . . . . . . . . 130

6.4.1.2 Model Assumptions . . . . . . . . . . . . . . . 133

6.4.1.3 Results .................. 136

6.4.2 Further Simulations . . . . . . . . . . . . . . . . . 141 
7 Meteoroid Fragmentation in a Planetary Atmosphere $\quad 147$

7.1 Introduction . . . . . . . . . . . . . . . . 147

7.2 The Fragmentation Process . . . . . . . . . . . . . . . . . . 149

7.2.1 Generation of Fragment Masses . . . . . . . . . . . . . . . . . 149

7.2.2 Initial Fragment Positions . . . . . . . . . . . . . . . . . 150

7.2.3 Fragment Separation Velocities . . . . . . . . . . . . 151

7.3 Modeling of Aerodynamic Interactions . . . . . . . . . . . . . 156

7.4 The Sikhote-Alin Crater Field . . . . . . . . . . . . . . . . 158

7.5 Results . . . . . . . . . . . . . . . . . . 161

7.5.1 Conditions During Aerodynamic Interactions . . . . . . . . . . 161

7.5.2 The Crater Field . . . . . . . . . . . . . . . . . 163

7.5.3 Statistical Analysis of Crater Distributions . . . . . . . . . 166

7.5.3.1 Overall Crater Field Shape . . . . . . . . . 166

7.5.3.2 Crater Groupings and Pair-wise Statistics . . . . . 167

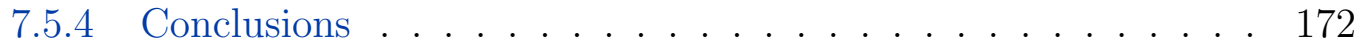

8 Conclusions $\quad 175$

$\begin{array}{ll}\text { Bibliography } & 179\end{array}$

A Force Coefficients for Gaussian Pressure Distribution 184

$\begin{array}{ll}\text { B T5 Run Conditions } & 187\end{array}$

C Calculation of the Drag Coefficient in the Impinging Case 189 


\section{List of Figures}

1.1 A sphere in hypersonic flow . . . . . . . . . . . . . . 2

1.2 Regions of interest in the proximal bodies problem . . . . . . . . . . 3

1.3 Shadowgraph of a broken projectile in the T5 light gas gun . . . . . . . 6

2.1 The axisymmetric blast wave analogy . . . . . . . . . . . . . . . 12

2.2 Variables in the planar blast wave analogy . . . . . . . . . . . . 15

2.3 Mach number and flow angle in the planar blast wave analogy . . . . . 16

2.4 Theoretical and computed pressure distributions on a hypersonic cylinder 19

2.5 Pressure coefficient at the stagnation point of a secondary cylinder . . 20

2.6 Theoretical secondary drag and lift coefficients in two dimensions (i) . 25

2.7 Theoretical secondary drag and lift coefficients in two dimensions (ii) . 26

2.8 Critical diameter ratio in two dimensions . . . . . . . . . . . . . 27

2.9 Variables in the axisymmetric blast wave analogy . . . . . . . . . . . 30

2.10 Mach number and flow angle in the axisymmetric blast wave analogy . 31

2.11 Theoretical and computed pressure distributions on a hypersonic sphere 32

2.12 Pressure coefficient at the stagnation point of a secondary sphere . . . 33

2.13 Theoretical secondary drag and lift coefficients in three dimensions (i) . 37

2.14 Theoretical secondary drag and lift coefficients in three dimensions (ii) 38

2.15 Critical diameter ratio in three dimensions . . . . . . . . . . . . . . . 39

3.1 Flow development in a two-dimensional computation . . . . . . . . . 44

3.2 Computational drag and lift coefficient profiles in two dimensions (i) . 46 
3.3 Computational drag and lift coefficient profiles in two dimensions (ii) . 47

3.4 Comparison of lift and drag coefficients obtained with Amrita and AMROC 50

3.5 Flow development in a three-dimensional computation . . . . . . . . 55

3.6 Computational drag and lift coefficient profiles in three dimensions (i) 56

3.7 Computational drag and lift coefficient profiles in three dimensions (ii) 58

3.8 Drag and lift coefficients in three dimensions at differing refinement levels 59

4.1 Theoretical and computed drag and lift coefficients in two dimensions (i) 63

4.2 Theoretical and computed coefficients in two dimensions (ii) . . . . . 65

4.3 Theoretical and computed coefficients in two dimensions (iii) . . . . . 66

4.4 Theoretical and computed coefficients in two dimensions (iv) . . . . . 67

4.5 Theoretical and computed coefficients in two dimensions (v) . . . . 68

4.6 Theoretical and computed drag and lift coefficients in three dimensions

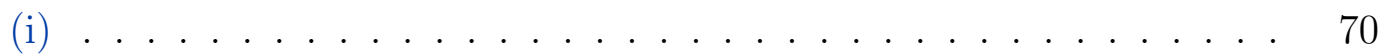

4.7 Theoretical and computed coefficients in three dimensions (ii) . . . . . 71

4.8 Theoretical and computed coefficients in three dimensions (iii) . . . . 72

4.9 Theoretical and computed coefficients in three dimensions (iv) . . . . 73

4.10 Theoretical and computed coefficients in three dimensions (v) . . . . . 74

5.1 Schematic sectional diagram of the T5 facility . . . . . . . . 78

5.2 Cutaway of the T5 test section showing model arrangement . . . . . 82

5.3 Photographs of model arrangements . . . . . . . . . . . . 82

$5.4 \quad$ Schematic of secondary model used for force measurements . . . . . . . 83

5.5 Power spectrum and acceleration signal recorded by accelerometer . . . 84

5.6 T5 optical setup for high-speed camera visualisation . . . . . . . . 86

5.7 Displacement profiles for shot $2330 \ldots \ldots$. . . . . . . . . 89

$5.8 \quad$ Schlieren images from shots 2320,2322 and 2325 . . . . . . . . 91

$5.9 \quad$ Experimental and computational schlieren images of shot $2326 \ldots$. . 97 
xiii

5.10 Experimental and computational schlieren images of shot $2328 \ldots$. . 98

5.11 Experimental and computational schlieren images of shot 2329 . . . . 99

5.12 Experimental and computational schlieren images of shot 2330 . . . 100

5.13 Acceleration, velocity and displacement of sphere . . . . . . . . . . 102

5.14 Pitot pressure histories for shots 2322 and $2324 \ldots \ldots$. . . . . . 104

5.15 Computational schlieren images of a sphere with supporting components 107

5.16 Plots of reference point displacements . . . . . . . . . . . . . 109

5.17 Primary sphere displacement and pitot pressure . . . . . . . . . . . . 112

6.1 Shock shapes and shock angles given by the blast wave analogy and

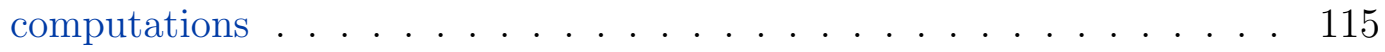

6.2 Flow angle and stagnation pressure coefficient profiles immediately behind bow shock . . . . . . . . . . . . . . . . . . . . 119

6.3 Approximation to flow geometry at $r=R_{s} \ldots \ldots . \ldots 121$

6.4 Computed and theoretical force coefficients at $r=R_{s} \ldots \ldots$. . . . 124

6.5 Approximation to the impinging flow geometry for $r<R_{s} \ldots \ldots$

6.6 Force coefficients for computations and the extended blast wave methodology. . . . . . . . . . . . . . . . . . . . . 128

6.7 Initial binary system configuration for atmospheric entry . . . . . . . . 131

6.8 Relative crater displacements for Earth entry of asteroid 2000 DP107 (i) 137

6.9 Relative crater displacements for Earth entry of asteroid 2000 DP107 (ii) 138

6.10 Relative crater displacements for Earth entry of asteroid 2000 DP107 (iii) 139

6.11 Relative crater displacements for Earth entry of other binary system (i) 143

6.12 Relative crater displacements for Earth entry of other binary system (ii) 144

6.13 Relative crater displacements for Earth entry of other binary system (iii) 145

6.14 Relative crater displacements for Earth entry of other binary system (iv) 146

7.1 Arrangement procedure for spherical fragments . . . . . . . . . . . . 152 
xiv

7.2 Effect of fragment number on arrangement procedure . . . . . . . . 153

7.3 Flowchart of fragmentation procedure . . . . . . . . . . . . . 159

7.4 Histograms of Mach numbers, downstream distances and relative speeds during interactions . . . . . . . . . . . . . . . . 162

7.5 Crater locations for simulated falls (i) . . . . . . . . . . . . 164

7.6 Crater locations for simulated falls (ii) . . . . . . . . . . . 165

7.7 Scatter plots of downrange and cross-range fragment displacements . . 168

7.8 Mean downrange and cross-range displacements for grouped fragments 169

7.9 Mean inter-fragment distances for simulated falls (i) . . . . . . . . . 171

7.10 Mean inter-fragment distances for simulated falls (ii) . . . . . . . . . . 172 


\section{List of Tables}

2.1 Values of $\eta_{0}$ in planar geometry for various values of $\gamma \ldots \ldots$

2.2 Values of $\eta_{0}$ in axisymmetric geometry for various values of $\gamma \ldots 28$

3.1 Details of two-dimensional AMROC computations. . . . . . . . . . . 42

3.2 Results of two-dimensional refinement study . . . . . . . . . . . . 49

3.3 Details of three-dimensional computations . . . . . . . . . . . . 53

3.4 Results of three-dimensional refinement study . . . . . . . . . . . . 60

$5.1 \quad$ Parameters for the technique validation experiments. . . . . . . . . . 90

5.2 Results from proximal bodies experiments . . . . . . . . . . . . 95

5.3 Results from simulations with model support components in $\mathrm{CO}_{2}$. . . 106

5.4 Results from simulations with model support components in $\mathrm{N}_{2}$. . . 106

7.1 Significance levels for $t$ tests comparing mean inter-fragment distances in interacting and non-interacting simulated falls . . . . . . . . . . . 173

B.1 Operating and stagnation conditions for T5 shots . . . . . . . . . 187

B.2 Freestream flow conditions in the T5 test section . . . . . . . . 188

B.3 Test gas concentrations in the T5 test section . . . . . . . . 188 


\section{Chapter 1}

\section{Introduction}

\subsection{Problem Definition and Scope of Current Work}

There are several important situations, in both the man-made and natural worlds, in which separate bodies travelling at hypersonic speeds may interact with one another aerodynamically. Launch vehicle stage separation, re-entry of multiple vehicles or a single vehicle with a trailing ballute, and a hypersonic vehicle launching some form of payload are all man-made situations in which the problem of proximal bodies in hypersonic flow may arise. In the natural world, such examples as a binary asteroid system entering a planetary atmosphere and meteoroid fragments interacting after atmospheric breakup may be cited. The current investigation will seek to better understand and quantify the forces at work in such situations.

Figure 1.1 shows a computational schlieren image of the top half of a sphere in a uniform freestream of Mach number $M_{\infty}=10$. This visualisation was produced by a simulation of the given configuration using the Amrita software system (Quirk, 1998). The axisymmetric two-dimensional Euler equations were solved using a finitevolume method - such a solution technique is typical in this flow regime. The Euler equations may often be used in place of the full Navier-Stokes equations in determining aerodynamic forces in hypersonic blunt body flows, in which most of the vorticity is generated by curved shocks and viscous forces may often be neglected. 


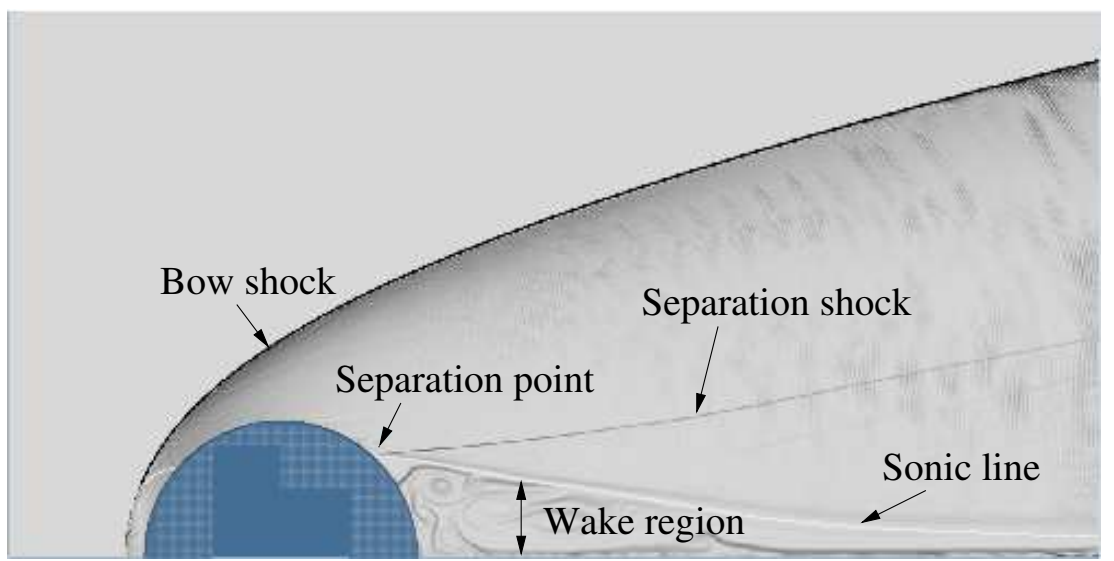

Figure 1.1: Computational schlieren image showing the top half of a sphere in a hypersonic freestream of Mach number $M_{\infty}=10$. Important flow features are indicated.

The main flow features are indicated on the image and are typical of blunt bodies in hypersonic flow in both two and three dimensions. A detached bow shock is generated, starting out as a normal shock just ahead of the stagnation point, and decaying to a Mach wave of angle $\beta=\arcsin \left(1 / M_{\infty}\right)$ in the far field. The distance from the stagnation point to the normal shock ahead is known as the shock stand-off distance and typically becomes very small at high Mach numbers. The flow separates from the rear surface of the sphere, creating a separation shock and wake region.

We now imagine placing a second body somewhere in this picture. Obviously, the aerodynamic effects - in particular, the nature of the forces - that this body will experience will depend very strongly on where the body is placed. We may identify at least four qualitatively different regions, as shown in Figure 1.2.

First, if the secondary body is placed immediately behind the primary body, it will find itself in the wake region, where, due to aerodynamic shielding from the primary body, the forces experienced will be small. Wake regions are also typically subject to unsteadiness, so we would expect time-variations to be present in the force history here. As the lateral displacement of the body is increased, it will move out of the aerodynamic shadow of the primary body and begin to experience the primary-shockprocessed flow. It is not immediately obvious what the nature of the forces will be 

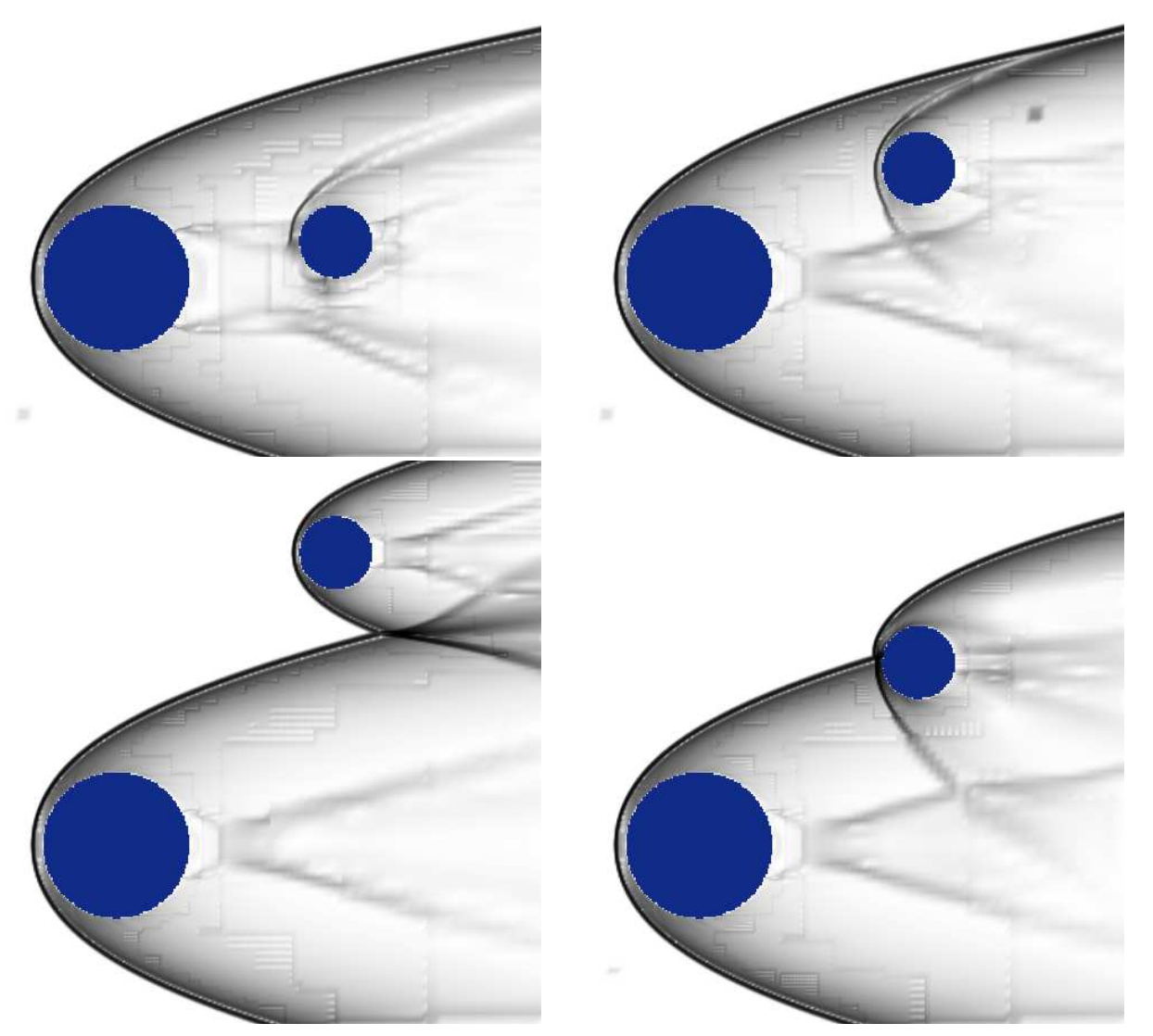

Figure 1.2: Regions of interest in the proximal bodies problem, clockwise from top left; the secondary body in the wake of the primary; the secondary body between the wake and primary bow shock; the primary bow shock impinging on the secondary body; the two bodies travelling independently but producing a shock-on-shock interaction

in this region; in particular, it is not obvious whether the body will experience an attractive or repulsive force from the primary axis of symmetry.

As the lateral displacement is increased further, the primary bow shock will begin to impinge upon the secondary body. In this region the drag force will be large, as the interaction between the two bow shocks produces a very high local pressure. We would also expect a repulsive force from the axis of symmetry to develop, as the outer part of the body will experience singly-shocked flow, whereas the inner part of the body will experience doubly-shocked - and thus higher pressure - flow. As the secondary 
body clears the bow shock it will begin to travel independently of the primary body. What might be of interest here, rather than the forces on the secondary body, is the nature of the shock-shock interactions that occur between the two bodies, especially if there are further bodies downstream that may be affected.

In this study, then, we will be primarily interested in investigating the forces on a secondary body placed within the second flow region as described above (i.e., that between the wake region and primary shock). We will also seek to better quantify the forces acting on the secondary body when the primary bow shock impinges upon it. In order to gain some general understanding of the effects at work in this problem, it will be necessary to restrict ourselves to simple body geometries. We shall thus limit our investigation to circular cylinders (in two dimensions) and spheres (in three dimensions). A three-pronged attack shall be mounted on the problem as follows:

1. The blast wave analogy from classical hypersonics is used to develop an analytic methodology to model both two- and three-dimensional versions of the problem.

2. The AMROC software is used to carry out ideal gas simulations of the two-body problem in both two and three dimensions.

3. Experiments are performed in the T5 hypervelocity shock tunnel to simulate the three-dimensional version of this problem. In order to determine the forces acting on the secondary body, a new force-measurement technique for shortduration hypersonic facilities is developed.

In addition, the analytic methodology will be used to model two physical problems. Firstly, the passage of a binary asteroid system through a planetary atmosphere will be simulated, and the effect of aerodynamic interactions on the relative body displacement at impact will be determined. Secondly, a model will be developed for the atmospheric fragmentation of a meteoritic body and the subsequent aerodynamic 
interactions of the fragments. In particular, we will seek to determine whether aerodynamic interactions could be responsible for the crater groupings that are observed in recent crater fields and have previously been attributed to secondary fragmentation.

\subsection{Review of Previous Work}

The previous work in this area has been concerned primarily with modeling the behaviour of a fragmented meteoroid in a planetary atmosphere. Among the first to investigate the problem were Passey and Melosh (1980), who looked at possible mechanisms for cross-range dispersion of fragments in crater fields. They concluded that the combined effects of bow shock interactions, crushing deceleration, and possibly spinning of the meteoroid were primarily responsible. They also obtained an estimate for the tranverse velocity of two fragments resulting from near-field shock interactions. Artem'eva and Shuvalov (1996) carried out numerical simulations of two fragments travelling in various relative configurations and found that when a secondary fragment was travelling within the shocked region created by a primary fragment, it experienced a force towards the axis of travel of the primary. In further simulations, Artemieva and Shuvalov (2001) found that this resulted in a collimation effect for a large number of fragments. These authors also developed a rough model to simulate the passage of a continually fragmenting body through the atmosphere. The collimation effect had previously been noted in experiments using the NASA-Ames Vertical Gun by Schultz and Sugita (1994).

A configuration very similar to the one that we will be investigating here has also been observed in the T5 hypervelocity shock tunnel. In Figure 1.3 is a shadowgraph taken by Lemieux (1999) during a series of experiments with the light gas gun modification of the T5. The projectile was broken during the acceleration phase, and a fragment is observed to travel within the shocked region created by the main body. 


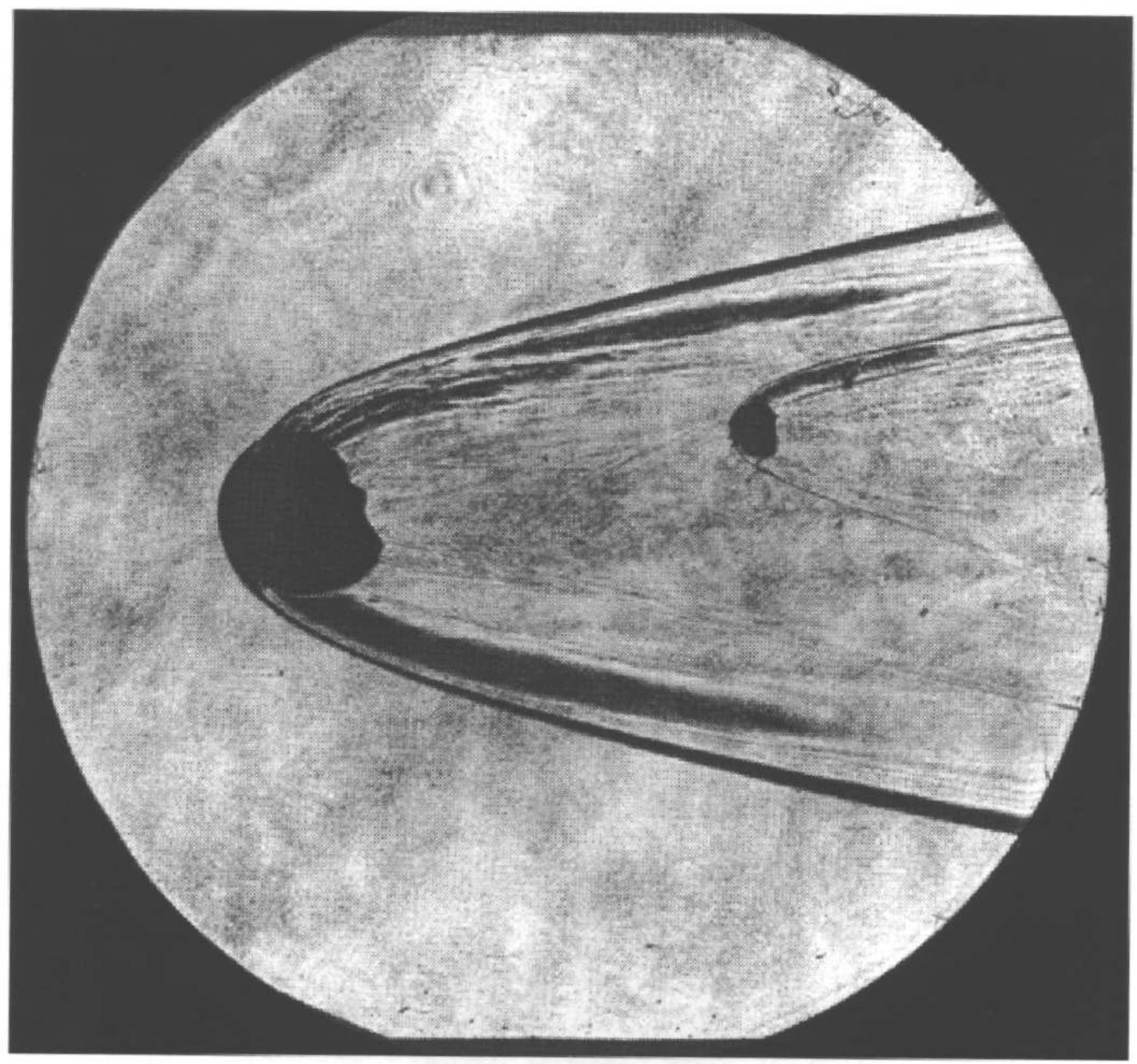

Figure 1.3: Shadowgraph of a broken spherical projectile in the light gas gun modification of the T5 hypervelocity shock tunnel.

\subsection{Discussion of Hypersonic Force-Measurement Techniques}

In order to carry out an experimental investigation of the proximal body problem, a new force-measurement technique had to be developed for use in the $\mathrm{T} 5$ hypervelocity shock tunnel. Traditionally, the measurement of forces and moments in high-enthalpy hypersonic wind tunnels, such as T5, has been restricted by the short test time intrinsic to these facilities. This flow time can be less than the period of the lowest natural 
frequency of a typical model and support, rendering measurement by conventional force balance techniques impractical. This has necessitated the development of other techniques. These may be loosely divided into two groups.

The first group consists of attempts to modify traditional force balance techniques so that they are more suited to short duration measurements. Storkmann et al. (1998) combined a model of high natural frequency (above $1 \mathrm{kHz}$ ) with accelerometers mounted in either the model or support to compensate for support oscillations. Measurements were made using a six-component strain gauge balance at two facilities: the Aachen shock tunnel TH2 and the Longshot facility at the von Karman Institute in Brussels. The success of this method appears to be highly dependent on model geometry, however. Results for a cone model showed good agreement with reference data, but agreement for a capsule model was less satisfactory, and the technique could not be applied to slender bodies, as such geometries are unable to accommodate internal mounting of the balance.

Another technique along these lines is the stress-wave force balance technique, first proposed by Sanderson and Simmons (1991). Instead of measuring steady-state forces, this technique involves the interpretation of stress waves induced within the model by aerodynamic loading. Extensive calibration is thus required to determine the response function of the system, introducing further error into the force-signal recovery. Mee (2003) claimed to have achieved 3\% accuracy in calibration studies for this technique and performed measurements in the T4 shock tunnel at the University of Queensland, Australia. These, however, were limited to a single component drag measurement - accuracy in earlier attempts at multi-component force measurement (Mee et al., 1996) were limited to 11\% accuracy. An attempt was recently made to apply this method to three-component force measurements on a large scramjet model (Robinson et al., 2004), but in this configuration the measurements were adversely affected by facility vibrations. Another limitation of this technique is the 
necessity of including a long stress-wave bar which places a constraint on the testable geometry.

The second group consists of techniques that make use of a support allowing for free-floating model behaviour during the test time. The short test time of the relevant facilities is actually an advantage for such techniques, as even for high loads the extent of motion of the model during the test time will be extremely small. Sahoo et al. (2003) implemented a method in which the model was mounted in flexible rubber bushes, allowing free-floating behaviour in flows of millisecond duration. The force and moments were measured during this period by means of embedded accelerometers. They achieved good agreement with theoretical values (typically 3-8\%) in a series of measurements on blunted cones in the HST2 hypersonic shock tunnel at the Indian Institute of Science, Bangalore. This is a relatively low-load facility, however - dynamic pressures in a facility such as the T5 are typically higher by an order of magnitude or more. It is thus questionable whether model motions in these facilities could be accommodated by such a setup. This technique also has the disadvantage of requiring extensive finite-element modeling, and the required mounting limits the geometries that may be simulated. Joarder and Jagadeesh (2004) implemented another free-floating technique in the HST2 facility, but this was limited to drag measurements.

Naumann et al. (1993) devised a method to allow for free-flight during the steady flow period whereby the model was mounted on a support that released just prior to the onset of the flow and tightened again shortly afterwards. Again, forces and moments were measured by means of accelerometers embedded in the model. This method relied on a cumbersome model support, however, which again limited the geometries that could be studied. Tanno et al. (2004) measured the forces generated by the interaction of a shock wave with a sphere in the vertical shock tube at the Interdisciplinary Shock Wave Research Center at Tohoku University. The sphere was 
suspended from a wire of sufficient length $(\sim 4 \mathrm{~m})$ that tensile stress waves in the wire had a negligible effect during the test time. Obviously, such a suspension method is not practical in a typical horizontal flow wind tunnel.

The technique that has been developed in the present work falls in this latter group. A relatively simple support system consisting of metal wires or cotton thread is used in conjunction with a catcher that halts the model motion after the end of the test time. Accelerations are recorded by an embedded accelerometer and by images taken with a high-speed digital camera. 


\section{Chapter 2}

\section{Application of the Blast Wave Analogy to Proximal Hypersonic Bodies}

\section{$2.1 \quad$ Introduction}

In this chapter we will develop an analytical methodology in both two and three dimensions to model the forces experienced by a secondary body when it is entirely within the shocked region created by a hypersonic primary body. To develop such a model, certain assumptions will need to be made about the body geometries. For reasons that will become apparent, we shall choose both bodies to be circular cylinders in two dimensions and spheres in three dimensions. In order to quantify the nature of the shocked region created by the primary body, we will make use of the blast wave analogy from classical hypersonics.

\subsection{The Blast Wave Analogy}

A similarity solution for the flow generated by a point explosion in an undisturbed atmosphere was first proposed by Taylor (1950) and famously applied to the New Mexico detonation of 1945. The explosion is idealized as instantaneously depositing a large energy $E$ into a perfect-gas atmosphere. The resulting flow is assumed to 
exhibit spherical symmetry and is bounded by a spherical shock wave expanding in the radial direction. Let the subscript $\infty$ denote conditions in the undisturbed atmosphere. If the shock is strong, defined by $U / a_{\infty} \gg 1$ (where $U$ is the shock velocity and $a$ the sound speed), then the ambient pressure $p_{\infty}$ may be neglected, and conditions immediately behind the shock (indicated by subscript $s$ ) are given by

$$
\begin{aligned}
& p_{s}=\frac{2}{\gamma+1} \rho_{\infty} U^{2}, \\
& u_{s}=\frac{2}{\gamma+1} U, \\
& \rho_{s}=\frac{\gamma+1}{\gamma-1} \rho_{\infty} .
\end{aligned}
$$

Using this strong shock assumption, Taylor was able to form a similarity variable involving only $\rho_{\infty}, E, r$, and $t$ and obtained a numerical solution to the problem. Full analytic solutions were subsequently obtained by Sedov (1959) in one, two, and three dimensions (corresponding to planar, circular, and spherical shocks, respectively).

If the shock is instead generated by a body travelling at speed $V$ in a direction which we assign to the (negative) $x$-axis, we may use dimensional similitude to replace $t$ in the point explosion solution by $x / V$. This is the so-called blast wave analogy, with planar and circular shocks analogous to plane and axisymmetric bodies, respectively. The explosion energy $E$ is equated with the work done by the drag force of the body. The strong shock assumption requires that the normal shock Mach number everywhere be large and so is formally valid in the limit $M_{\infty} \rightarrow \infty$ where $M_{\infty}=V / a_{\infty}$. For finite (but large) $M_{\infty}$, this assumption will become less accurate at large values of $x / V$. A graphical representation of the axisymmetric blast wave analogy is shown in Figure 2.1. 

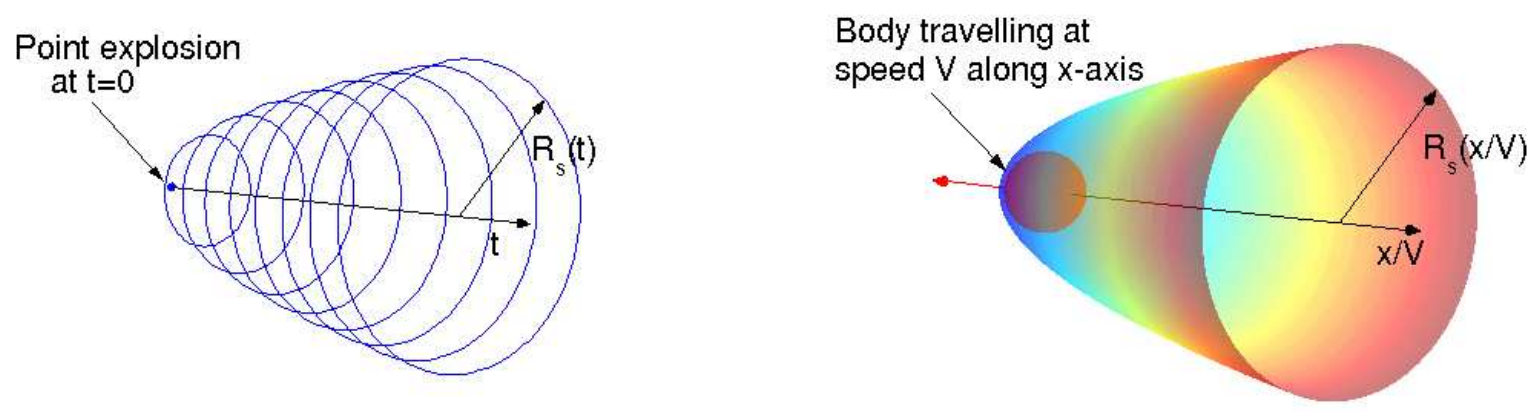

Figure 2.1: The axisymmetric blast wave analogy. The temporally-growing circular shock wave produced by a point explosion in two space dimensions, shown at various times (left), is analogous to the axisymmetric shock wave generated by a body travelling at hypersonic speeds (right).

\subsection{Modeling in Two Dimensions}

\subsubsection{The Planar Blast Wave Analogy}

A planar blast wave is analagous to the flow produced by a symmetric two-dimensional body. The similarity variable in this case is

$$
\eta=\left(\frac{\rho_{\infty}}{E}\right)^{1 / 3} \frac{r}{t^{2 / 3}} \longrightarrow\left(\frac{2}{A C_{D}}\right)^{1 / 3} \frac{r}{x^{2 / 3}}
$$

where $\rho_{\infty}$ is the ambient density and $E$ is the energy released, per unit area, in the analogous explosion. $A$ and $C_{D}$ are the projected frontal surface area per unit depth and drag coefficient of the body, respectively. We have equated $E$ with the drag force, per unit depth, on the body, given by $\frac{1}{2} C_{D} \rho_{\infty} V^{2} A$. We assume the body to be 
a circular cylinder, and so replace $A$ with $d_{1}$, the body diameter. The drag coefficient is given a value of 1.2 in this exposition, which is appropriate for a cylinder in high Mach number flow. As may be seen, however, dependence on the drag coefficient is relatively weak.

The shock radius $R_{s}$ is then given by

$$
R_{s}=\eta_{0}\left(\frac{d_{1} C_{D}}{2}\right)^{1 / 3} x^{2 / 3}
$$

where $\eta_{0}$ is a constant that depends on the ratio of specific heats, $\gamma$. It may be shown to take the values given in Table 2.1. Details of the calculation of $\eta_{0}$ may be found in Landau and Lifshitz (1989).

\begin{tabular}{c|c|c|c}
$\gamma$ & 1.30 & 1.40 & 1.67 \\
\hline$\eta_{0}$ & 0.8805 & 0.9756 & 1.1861
\end{tabular}

Table 2.1: Values of $\eta_{0}$ in planar geometry for various values of $\gamma$

The nondimensional dependent variables are

$$
\begin{aligned}
& \hat{p}=\frac{9(\gamma+1)}{8} \frac{p}{\rho_{\infty}(r V / x)^{2}}, \\
& \hat{u}=\frac{3(\gamma+1)}{4} \frac{u}{r V / x}, \\
& \hat{\rho}=\frac{\gamma-1}{\gamma+1} \frac{\rho}{\rho_{\infty}},
\end{aligned}
$$

where $u$ is the radial velocity. The constants have been introduced to simplify the boundary conditions at $r=R_{s}$ (i.e., $\hat{p}=\hat{u}=\hat{\rho}=1$ at $r=R_{s}$ ). 
The equations of motion for the gas flow behind the shock are

$$
\begin{array}{r}
u \frac{\partial \rho}{\partial r}+V \frac{\partial \rho}{\partial x}+\rho \frac{\partial u}{\partial r}=0 \\
u \frac{\partial u}{\partial r}+V \frac{\partial u}{\partial x}+\frac{1}{\rho} \frac{\partial p}{\partial r}=0 \\
\left(u \frac{\partial}{\partial r}+V \frac{\partial}{\partial x}\right) \log \frac{p}{\rho^{\gamma}}=0 .
\end{array}
$$

The first two equations are conservation of mass and momentum, respectively, while the third is the condition that the entropy is constant along a streamline. Upon substitution of the dimensionless variables into Equations 2.9 through 2.11, the following solution may be obtained:

$$
\begin{aligned}
\left(\frac{\eta_{0}}{\eta}\right)^{3} & =\hat{u}^{2}\left(\frac{2 \gamma \hat{u}-(\gamma+1)}{\gamma-1}\right)^{k_{1}}(3-2 \hat{u})^{k_{2}} \\
\hat{\rho} & =\left(\frac{\gamma+1-2 \hat{u}}{\gamma-1}\right)^{k_{3}}\left(\frac{2 \gamma \hat{u}-(\gamma+1)}{\gamma-1}\right)^{k_{4}}(3-2 \hat{u})^{k_{5}} \\
\frac{\hat{p}}{\hat{\rho}} & =\frac{\hat{u}^{2}(\gamma+1-2 \hat{u})}{2 \gamma \hat{u}-(\gamma+1)}
\end{aligned}
$$

where

$$
\begin{aligned}
& k_{1}=-\frac{3(\gamma-1)}{2 \gamma-1} \\
& k_{2}=\frac{5 \gamma^{2}+\gamma-4}{(\gamma+1)(2 \gamma-1)} \\
& k_{3}=-\frac{2}{2-\gamma} \\
& k_{4}=\frac{1}{2 \gamma-1} \\
& k_{5}=\frac{5 \gamma^{2}+\gamma-4}{(\gamma+1)(2-\gamma)(2 \gamma-1)} .
\end{aligned}
$$

A derivation of the general solution (in one, two, or three dimensions) may be found in Hayes and Probstein (1966). Plots of the dimensional form of the solution 
variables, normalized by their values immediately behind the shock, are shown in Figure 2.2. A value of $\gamma=1.4$ has been chosen and shall be assumed in that which follows.

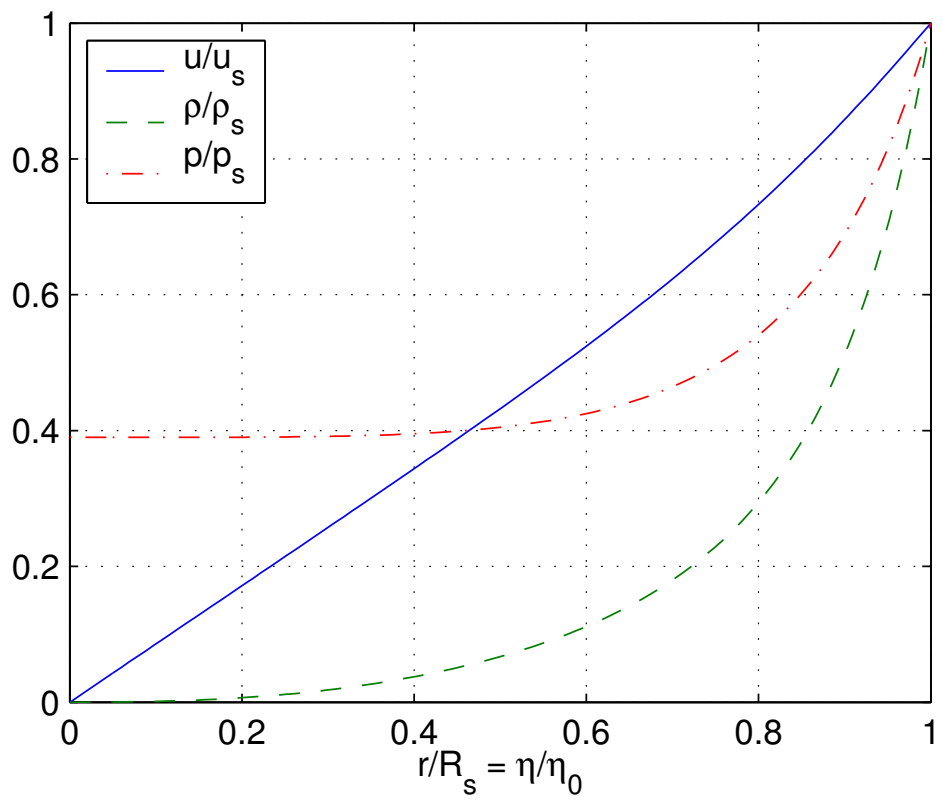

Figure 2.2: Variables in the planar blast wave analogy for a ratio of specific heats of $\gamma=1.4$

The Mach number $M$ and flow angle $\delta$ may also be calculated:

$$
\begin{aligned}
M & =\frac{\sqrt{u^{2}+V^{2}}}{\sqrt{\gamma p / \rho}} \\
& =\left(\frac{\frac{9}{8}\left(\frac{\gamma+1}{\eta}\right)^{2}\left(\frac{2}{C_{D}} \frac{x}{d_{1}}\right)^{2 / 3}+2 \hat{u}^{2}}{\gamma(\gamma-1) \hat{p} / \hat{\rho}}\right)^{\frac{1}{2}} \\
\delta & =\arctan \left(\frac{u}{V}\right) \\
& =\arctan \left(\frac{4}{3(\gamma+1)}\left(\frac{2}{C_{D}} \frac{x}{d_{1}}\right)^{-1 / 3} \eta \hat{u}\right) .
\end{aligned}
$$

Plots of these variables are shown in Figure 2.3. Note that the Mach number at a given value of $\eta$ will increase without bound as $x / d_{1} \rightarrow \infty$. 

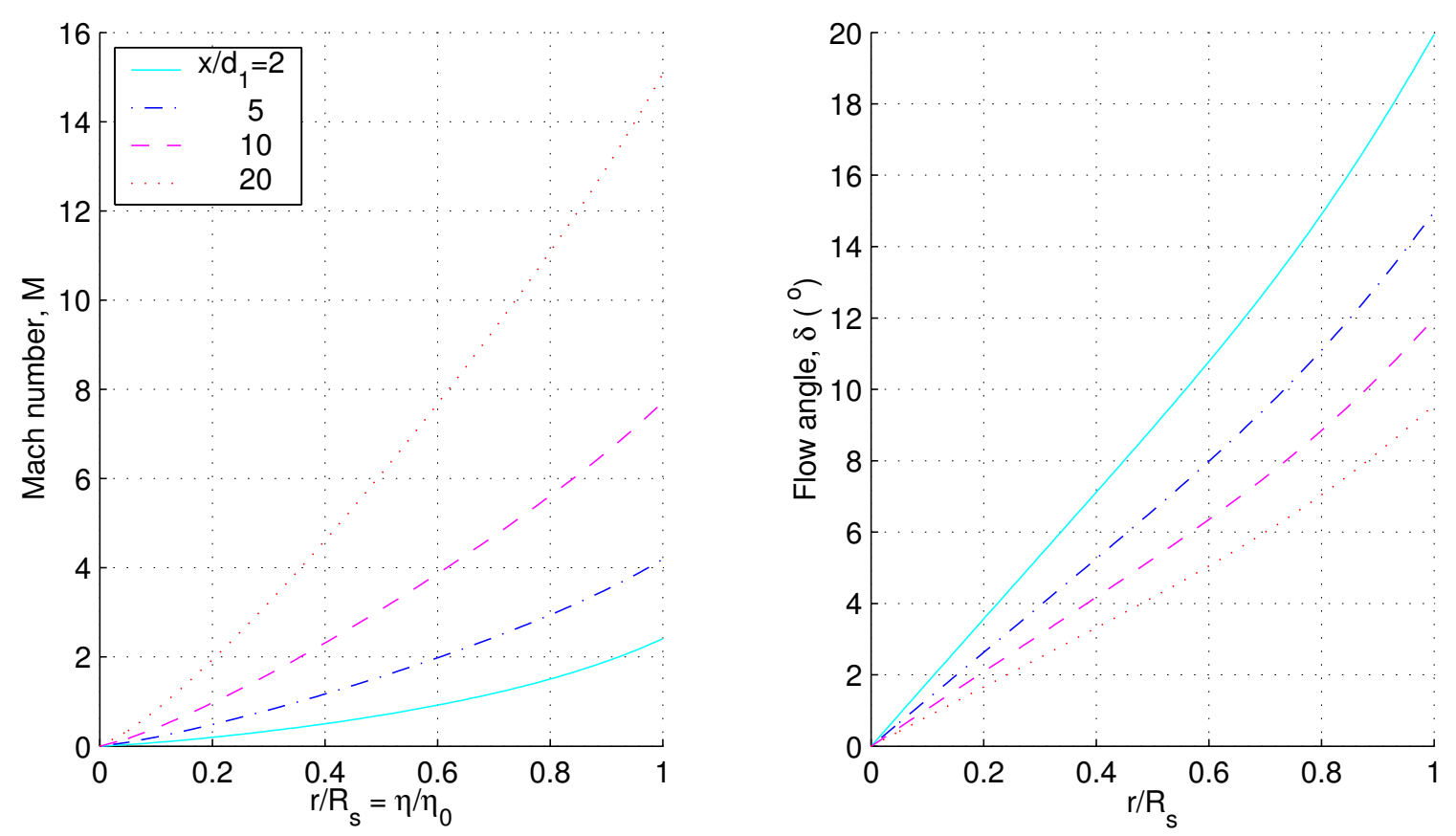

Figure 2.3: Mach number and flow angle profiles at various distances downstream in the planar blast wave analogy

\subsubsection{Proximal Bodies in Two Dimensions}

\subsubsection{Pressure Distribution on the Secondary Body}

We now imagine placing a second body at some point inside the shocked region described by the planar blast wave analogy. We specify this also to be a circular cylinder and assume that it is placed above the plane of symmetry of the primary body, so a positive lift coefficient indicates a repulsive force from this plane. We also assume that the secondary body is stationary with respect to the primary body. The forces (lift and drag) acting on this body may be obtained by integrating the pressure with the appropriate component of the normal vector over the surface of the body. We thus require an estimate of the pressure distribution over the body.

To this end, let us first consider the same body travelling in the uniform supersonic freestream outside the shocked region. The pressure distribution corresponding to the 
Newtonian flow solution, valid in the double limit $M_{\infty} \rightarrow \infty, \gamma \rightarrow 1$, is

$$
C_{p}(\theta)= \begin{cases}2 \cos ^{2} \theta & \text { for }|\theta|<\pi / 2, \\ 0 & \text { for } \pi / 2 \leq|\theta|<\pi\end{cases}
$$

where $C_{p}(\theta)=\left(p(\theta)-p_{\infty}\right) / \frac{1}{2} \rho_{\infty} V^{2}$ and $\theta$ is the angle measured from the stagnation point. For finite $M_{\infty}$ and $\gamma>1$, an improved estimate is possible if we keep the $\cos ^{2} \theta$ dependence but fix the value of the pressure at the stagnation point with our knowledge of the flow processes along the stagnation streamline. The fluid will pass through a normal shock and then decelerate to the stagnation point. If the subscripts 2 and stag refer to conditions immediately behind the normal shock and at the stagnation point, respectively, we have for a inviscid, perfect gas:

$$
\begin{aligned}
\frac{p_{\text {stag }}}{p_{\infty}} & =\frac{p_{2}}{p_{\infty}} \frac{p_{\text {stag }}}{p_{2}} \\
& =\left(1+\frac{2 \gamma}{\gamma+1}\left(M_{\infty}^{2}-1\right)\right)\left(1+\frac{\gamma-1}{2} M_{2}^{2}\right)^{\frac{\gamma}{\gamma-1}}
\end{aligned}
$$

with

$$
M_{2}^{2}=\frac{(\gamma-1) M_{\infty}^{2}+2}{2 \gamma M_{\infty}^{2}-(\gamma-1)},
$$

and we have for the $|\theta|<\pi / 2$ component of our distribution the modified Newtonion profile $p(\theta)=\left(p_{\text {stag }}-p_{\infty}\right) \cos ^{2} \theta+p_{\infty}$. For $|\theta| \geq \pi / 2, p(\theta)=p_{\infty}$, as before. We have dropped the $C_{p}$ notation, as it is somewhat unwieldy in this case.

The normalized profile, $p(\theta) / p_{\text {stag }}$, given by this description is plotted in Figure 2.4 for two different Mach numbers. These are compared with profiles computed using the Amrita software system (details of numerical simulations are given in Chapter 3). These computational profiles have been normalized by the computed pressures at the stagnation point, which differ slightly from the theoretical values. The Gaussian profile $p(\theta)=p_{\text {stag }} \exp \left(-\theta^{2}\right)$ is also included, as this was found to give very good 
agreement with the computed distributions ahead of the respective separation points. Neither theoretical profile is able to capture the pressure jumps aft of the separation points, but the Gaussian profile does particularly poorly in this region due to the decaying exponential tail. As may be seen, however, this pressure jump becomes less significant as the freestream Mach number is increased, so we might expect the Gaussian profile to be most appropriate at high Mach numbers. We shall consider both the modified Newtonian and Gaussian profiles as reference distributions, and, defining the pressure coefficient $p^{\prime}=p / \frac{1}{2} \rho_{\infty} V^{2}$, we write the modified Newtonian profile as

$$
p^{\prime}(\theta)= \begin{cases}\left(p_{\text {stag }}^{\prime}-p_{\infty}^{\prime}\right) \cos ^{2} \theta+p_{\infty}^{\prime} & \text { for }|\theta|<\pi / 2, \\ p_{\infty}^{\prime} & \text { for } \pi / 2 \leq|\theta|<\pi\end{cases}
$$

and the Gaussian profile as

$$
p^{\prime}(\theta)=p_{\text {stag }}^{\prime} \exp \left(-\theta^{2}\right) \text { for }|\theta|<\pi
$$

We now wish to use these reference distributions to approximate the pressure distribution on the cylinder inside the shocked region. Thus, we redefine $p_{\text {stag }}$ as the pressure obtained by passing the flow at some point within the shocked region through a normal shock, followed by an isentropic deceleration to zero velocity. If the subscript 1 denotes conditions at a point in the blast wave solution, Equations 2.18 and 2.19 will now hold for $p_{\text {stag }}$ if we replace $p_{\infty}$ and $M_{\infty}$ by $p_{1}$ and $M_{1}$, respectively. Similarly, the modified Newtonian profile 2.20 will be appropriate for this region if $p_{\infty}$ is replaced by $p_{1}$; the Gaussian profile 2.21 carries over directly with our new definition of $p_{\text {stag. }}$. Note that in our normalization of the pressures, however, we still use $\frac{1}{2} \rho_{\infty} V^{2}$ rather than $\frac{1}{2} \rho_{1} V^{2}$.

We now encounter a problem that was not present in the freestream case. The 

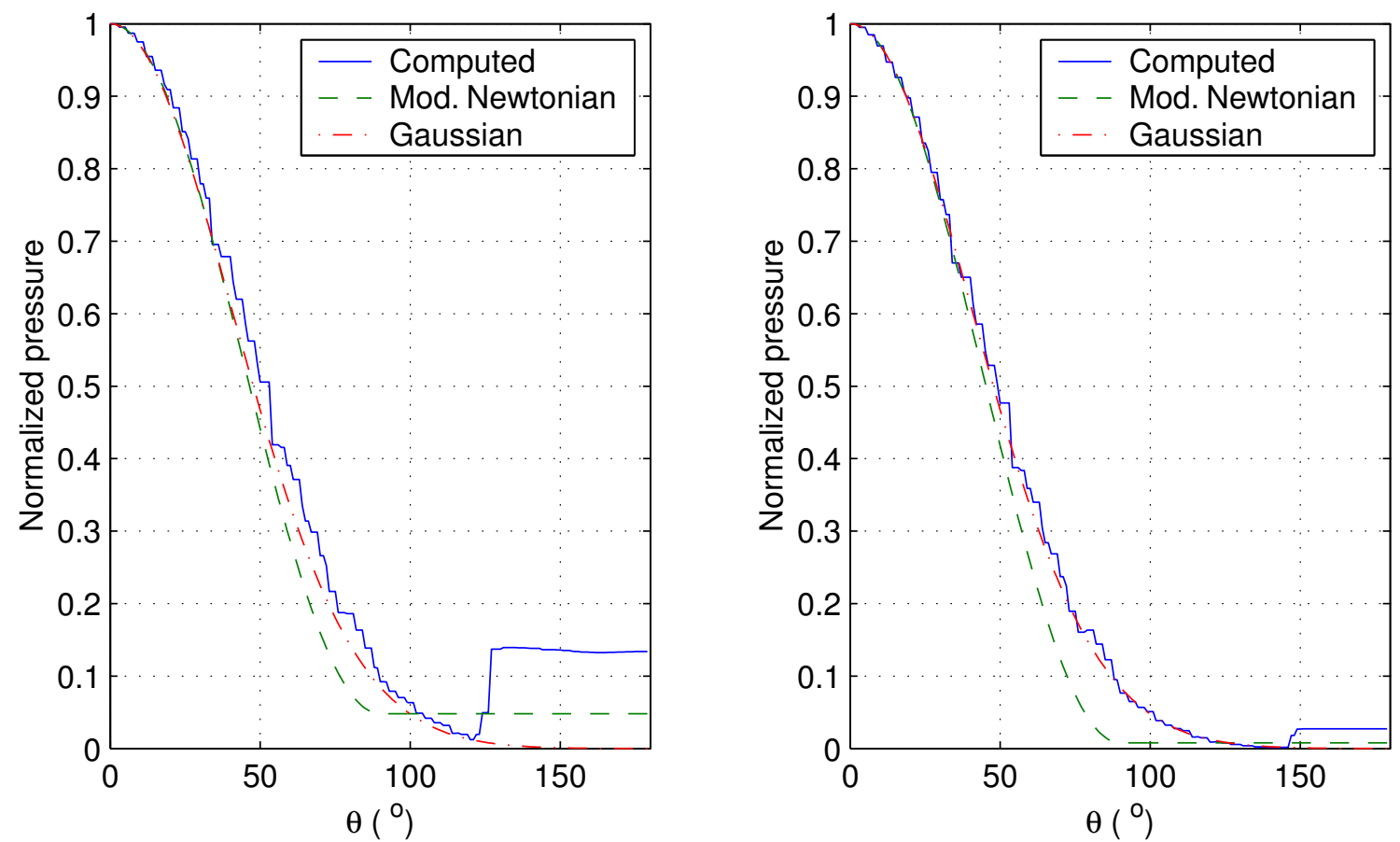

Figure 2.4: Theoretical and computed normalized pressure distributions on a cylinder in a uniform freestream of $M_{\infty}=4$ (left) and $M_{\infty}=10$ (right)

variables $p_{\text {stag }}, p_{1}$, and $M_{1}$ are not constant in space and hence will vary over the area occupied by the secondary body. We choose as our reference point for these variables the center of the secondary body.

The first deviation from the redefined reference distributions that we wish to account for is that the flow experienced by the secondary body will no longer be aligned with the plane of the primary body's travel but will be deflected by an angle $\delta$, given by Equation 2.16. This will have the effect of shifting the stagnation point towards the underside of the cylinder, resulting in a decrease in drag and a positive lift contribution. Here our choice of the secondary body as a circular cylinder becomes important - the rotational symmetry possessed by this shape make this deviation easy to account for.

Second, noting from Figure 2.5 that $p_{\text {stag }}^{\prime}$ varies strongly with $r / R_{s}$, we wish to account for the variation of $p_{\text {stag }}^{\prime}$ over the area covered by the cylinder. For example, 


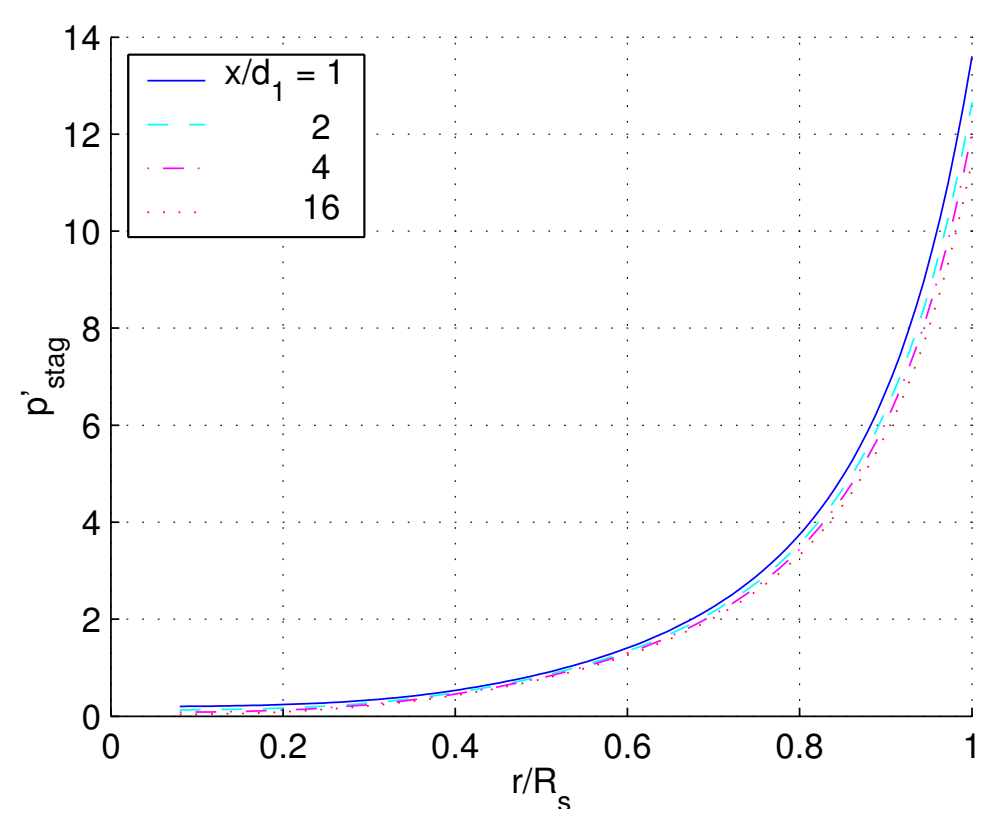

Figure 2.5: Pressure coefficient $p_{\text {stag }}^{\prime}$ at the stagnation point of a secondary body placed at various distances downstream within the shocked region.

noting from Figure 2.5 that $p_{\text {stag }}^{\prime}$ increases strongly in the radial direction, the effective stagnation pressure on the upper side of the secondary body will be greater than that on the lower side. This will result in a negative contribution to the lift. Let us then examine which variations we need to consider. As the profile varies only very little with downstream displacement, variation of $p_{\text {stag }}^{\prime}$ with $x$ will be due almost entirely to variation of $R_{s}$ with $x$. As $R_{s} \propto x^{2 / 3}$, the dependence of $p_{\text {stag }}^{\prime}$ on $x$ will be weaker than that on $r$. Also, as the flow is principally in the $x$ direction, it is not clear that we can legitimately localize $x$-variations over the body. Thus, we shall include variations of $p_{\text {stag }}^{\prime}$ with $x$ for the sake of completeness, but with the understanding that they are not indispensible to our model.

We may estimate the variations by Taylor-series expanding $p_{\text {stag }}$ to the linear term in $r$ and $x$. If $d_{2}$ is the diameter of the secondary body, the $x$ and $r$ displacements of a point on the cylinder's surface are given by $d_{2} / 2 \cos \theta$ and $d_{2} / 2 \sin \theta$, respectively. We then make the approximation that the effective stagnation pressure, $p_{\text {stag }}^{\text {eff }}$, at a 
point on the cylinder's surface is given by

$$
p_{\text {stag }}^{\text {eff }}=p_{\text {stag }}+\frac{1}{2} \frac{d_{2}}{d_{1}}\left(\frac{\partial p_{\text {stag }}}{\partial\left(r / d_{1}\right)} \sin \theta+\frac{\partial p_{\text {stag }}}{\partial\left(x / d_{1}\right)} \cos \theta\right),
$$

where $\partial p_{\text {stag }} / \partial\left(r / d_{1}\right)$ and $\partial p_{\text {stag }} / \partial\left(x / d_{1}\right)$ may be determined by differentiating Equation (2.18). All lengths have been non-dimensionalized by the primary body diameter $d_{1}$

\subsubsection{Drag and Lift Coefficients}

The drag and lift coefficients of the secondary body are given by

$$
\begin{aligned}
C_{D} & =\frac{1}{2} \int_{-\pi}^{\pi} p^{\prime}(\theta) \cos \theta d \theta \\
C_{L} & =-\frac{1}{2} \int_{-\pi}^{\pi} p^{\prime}(\theta) \sin \theta d \theta .
\end{aligned}
$$

Combining the effects described above, these may be written for the modified Newtonian case as

$$
\begin{aligned}
C_{D} & =\frac{1}{2} \int_{-\pi / 2}^{\pi / 2}\left(p_{\text {stag }}^{\prime}-p_{1}^{\prime}+\frac{1}{2} \frac{d_{2}}{d_{1}} \frac{\partial\left(p_{\text {stag }}^{\prime}-p_{1}^{\prime}\right)}{\partial\left(r / d_{1}\right)} \sin (\theta-\delta)\right. \\
& \left.-\frac{1}{2} \frac{d_{2}}{d_{1}} \frac{\partial\left(p_{\text {stag }}^{\prime}-p_{1}^{\prime}\right)}{\partial\left(x / d_{1}\right)} \cos (\theta-\delta)\right) \cos ^{2} \theta \cos (\theta-\delta) d \theta \\
& +\frac{1}{2} \int_{-\pi}^{\pi}\left(p_{1}^{\prime}+\frac{1}{2} \frac{d_{2}}{d_{1}} \frac{\partial p_{1}^{\prime}}{\partial\left(r / d_{1}\right)} \sin (\theta-\delta)\right. \\
& \left.-\frac{1}{2} \frac{d_{2}}{d_{1}} \frac{\partial p_{1}^{\prime}}{\partial\left(x / d_{1}\right)} \cos (\theta-\delta)\right) \cos (\theta-\delta) d \theta
\end{aligned}
$$


and

$$
\begin{aligned}
C_{L} & =-\frac{1}{2} \int_{-\pi / 2}^{\pi / 2}\left(p_{\text {stag }}^{\prime}-p_{1}^{\prime}+\frac{1}{2} \frac{d_{2}}{d_{1}} \frac{\partial\left(p_{\text {stag }}^{\prime}-p_{1}^{\prime}\right)}{\partial\left(r / d_{1}\right)} \sin (\theta-\delta)\right. \\
& \left.-\frac{1}{2} \frac{d_{2}}{d_{1}} \frac{\partial\left(p_{\text {stag }}^{\prime}-p_{1}^{\prime}\right)}{\partial\left(x / d_{1}\right)} \cos (\theta-\delta)\right) \cos ^{2} \theta \sin (\theta-\delta) d \theta \\
& -\frac{1}{2} \int_{-\pi}^{\pi}\left(p_{1}^{\prime}+\frac{1}{2} \frac{d_{2}}{d_{1}} \frac{\partial p_{1}^{\prime}}{\partial\left(r / d_{1}\right)} \sin (\theta-\delta)\right. \\
& \left.-\frac{1}{2} \frac{d_{2}}{d_{1}} \frac{\partial p_{1}^{\prime}}{\partial\left(x / d_{1}\right)} \cos (\theta-\delta)\right) \sin (\theta-\delta) d \theta
\end{aligned}
$$

For consistency, the derivatives of $p_{1}^{\prime}$ have also been included. For the Gaussian distribution:

$$
\begin{aligned}
C_{D} & =\frac{1}{2} \int_{-\pi}^{\pi}\left(p_{\text {stag }}^{\prime}+\frac{1}{2} \frac{d_{2}}{d_{1}} \frac{\partial p_{\text {stag }}^{\prime}}{\partial\left(r / d_{1}\right)} \sin (\theta-\delta)\right. \\
& \left.-\frac{1}{2} \frac{d_{2}}{d_{1}} \frac{\partial p_{\text {stag }}^{\prime}}{\partial\left(x / d_{1}\right)} \cos (\theta-\delta)\right) \exp \left(-\theta^{2}\right) \cos (\theta-\delta) d \theta \\
C_{L} & =-\frac{1}{2} \int_{-\pi}^{\pi}\left(p_{\text {stag }}^{\prime}+\frac{1}{2} \frac{d_{2}}{d_{1}} \frac{\partial p_{\text {stag }}^{\prime}}{\partial\left(r / d_{1}\right)} \sin (\theta-\delta)\right. \\
& \left.-\frac{1}{2} \frac{d_{2}}{d_{1}} \frac{\partial p_{\text {stag }}^{\prime}}{\partial\left(x / d_{1}\right)} \cos (\theta-\delta)\right) \exp \left(-\theta^{2}\right) \sin (\theta-\delta) d \theta
\end{aligned}
$$

For the modified Newtonian description, the integrals may be evaluated to yield

$$
\begin{aligned}
C_{D} & =\frac{2}{3} \cos \delta\left(p_{\text {stag }}^{\prime}-p_{1}^{\prime}\right)-\frac{\pi}{32} \frac{d_{2}}{d_{1}} \sin 2 \delta \frac{\partial\left(p_{\text {stag }}^{\prime}-p_{1}\right)}{\partial\left(r / d_{1}\right)} \\
& -\frac{\pi}{16} \frac{d_{2}}{d_{1}}(2+\cos 2 \delta) \frac{\partial\left(p_{\text {stag }}^{\prime}-p_{1}^{\prime}\right)}{\partial\left(x / d_{1}\right)}-\frac{\pi}{4} \frac{d_{2}}{d_{1}} \frac{\partial p_{1}^{\prime}}{\partial\left(x / d_{1}\right)} \\
C_{L} & =\frac{2}{3} \sin \delta\left(p_{\text {stag }}^{\prime}-p_{1}^{\prime}\right)-\frac{\pi}{32} \frac{d_{2}}{d_{1}} \sin 2 \delta \frac{\partial\left(p_{\text {stag }}^{\prime}-p_{1}^{\prime}\right)}{\partial\left(x / d_{1}\right)} \\
& -\frac{\pi}{16} \frac{d_{2}}{d_{1}}(2-\cos 2 \delta) \frac{\partial\left(p_{\text {stag }}^{\prime}-p_{1}^{\prime}\right)}{\partial\left(r / d_{1}\right)}-\frac{\pi}{4} \frac{d_{2}}{d_{1}} \frac{\partial p_{1}^{\prime}}{\partial\left(r / d_{1}\right)} .
\end{aligned}
$$

The integrals in the Gaussian case may also be evaluated, and the resulting expressions may be found in Appendix A. The error incurred by using the following 
approximations to these expressions is less than $0.01 \%$ :

$$
\begin{aligned}
C_{D} & \approx \frac{\sqrt{\pi}}{2}\left[e^{-1 / 4} p_{\text {stag }}^{\prime} \cos \delta-\frac{1}{4 e} \frac{d_{2}}{d_{1}} \frac{\partial p_{\text {stag }}^{\prime}}{\partial\left(r / d_{1}\right)} \sin 2 \delta\right. \\
& \left.-\frac{1}{4} \frac{d_{2}}{d_{1}}\left(1+\frac{1}{e} \cos 2 \delta\right) \frac{\partial p_{\text {stag }}^{\prime}}{\partial\left(x / d_{1}\right)}\right] \\
C_{L} & \approx \frac{\sqrt{\pi}}{2}\left[e^{-1 / 4} p_{\text {stag }}^{\prime} \sin \delta-\frac{1}{4 e} \frac{d_{2}}{d_{1}} \frac{\partial p_{\text {stag }}^{\prime}}{\partial\left(x / d_{1}\right)} \sin 2 \delta\right. \\
& \left.-\frac{1}{4} \frac{d_{2}}{d_{1}}\left(1-\frac{1}{e} \cos 2 \delta\right) \frac{\partial p_{\text {stag }}^{\prime}}{\partial\left(r / d_{1}\right)}\right] .
\end{aligned}
$$

As the diameter ratio is the only parameter we can freely choose in the coefficient expressions, it is of interest to ask what the effect of varying this parameter may be. We see that in each expression, all but one term contains the diameter ratio. Concentrating on the modified Newtonian coefficients, we assume in each case that this term is of the same order as the dominant term involving $d_{2} / d_{1}$. Noting then that over most of the shocked region $|\delta|$ is small and $p_{\text {stag }}^{\prime} \gg p_{1}^{\prime}$, we approximate the modified Newtonian formulae as

$$
\begin{aligned}
C_{D} & \approx \frac{2}{3} p_{\text {stag }}^{\prime}-\frac{3 \pi}{16} \frac{d_{2}}{d_{1}} \frac{\partial p_{\text {stag }}^{\prime}}{\partial\left(x / d_{1}\right)} \\
C_{L} & \approx \frac{2}{3} p_{\text {stag }}^{\prime} \sin \delta-\frac{\pi}{16} \frac{d_{2}}{d_{1}} \frac{\partial p_{\text {stag }}^{\prime}}{\partial(r / d 1)}
\end{aligned}
$$

The second term in the $C_{D}$ equation is positive in sign (as $\partial p_{\text {stag }}^{\prime} / \partial x<0$ ) and will serve to augment the first term, which is the freestream drag coefficient. In the $C_{L}$ equation the two terms are of opposite sign. As only the second depends on body size, we have the potential for a qualitative change of behaviour as the body size is varied. In particular, there will be a tendency for the lift coefficient to become more negative as the body size is increased. This is in fact precisely what is observed in Figures 2.6 and 2.7, in which the lift and drag coefficients (the full modified Newtonian expressions 2.29 and 2.30, rather than the approximations 2.33 
and 2.34) are plotted for different sized bodies at various distances downstream. The profiles in Figure 2.7 include the $x$-derivative terms, whereas those in Figure 2.6 do not. In both cases we see that the lift coefficient varies strongly with body size, as predicted. Note also the tendency for the lift coefficients to become more positive with increasing distance downstream, indicating that the second term in Equation 2.34 decays more rapidly than the first. In the cases in which $x$-derivatives are absent, the drag coefficient is hardly affected by either body size or distance downstream, whereas if they are included the drag drops gradually as the distance downstream is increased.

Having noted that the sign of the lift coefficient may, in general, depend on the ratio of sizes of the two bodies, we may wish to ask whether there is a critical diameter ratio above or below which the lift is exclusively of one sign throughout the shocked region. To determine whether this is the case, we set Equation 2.30 to zero and solve for $d_{2} / d_{1}$. The resulting curves, as functions of $r / R_{s}$, are plotted in Figure 2.8 for various distances downstream. At a given distance downstream, a secondary body with a diameter ratio lying below the corresponding curve will experience a positive lift coefficient, and thus a repulsive lateral force, while one lying above will experience a negative lift coefficient. As may be seen, however, the value of $d_{2} / d_{1}$ needed to produce an exclusively negative lift coefficient continues to rise as the downstream displacement is increased. This is consistent with our earlier observations. Also, the diameter ratio must become vanishingly small for the lift coefficient to be exclusively positive throughout the domain. We thus conclude that there is no critical value of the diameter ratio for which the secondary body will experience a lift coefficient of exclusively one sign throughout the shocked region. 

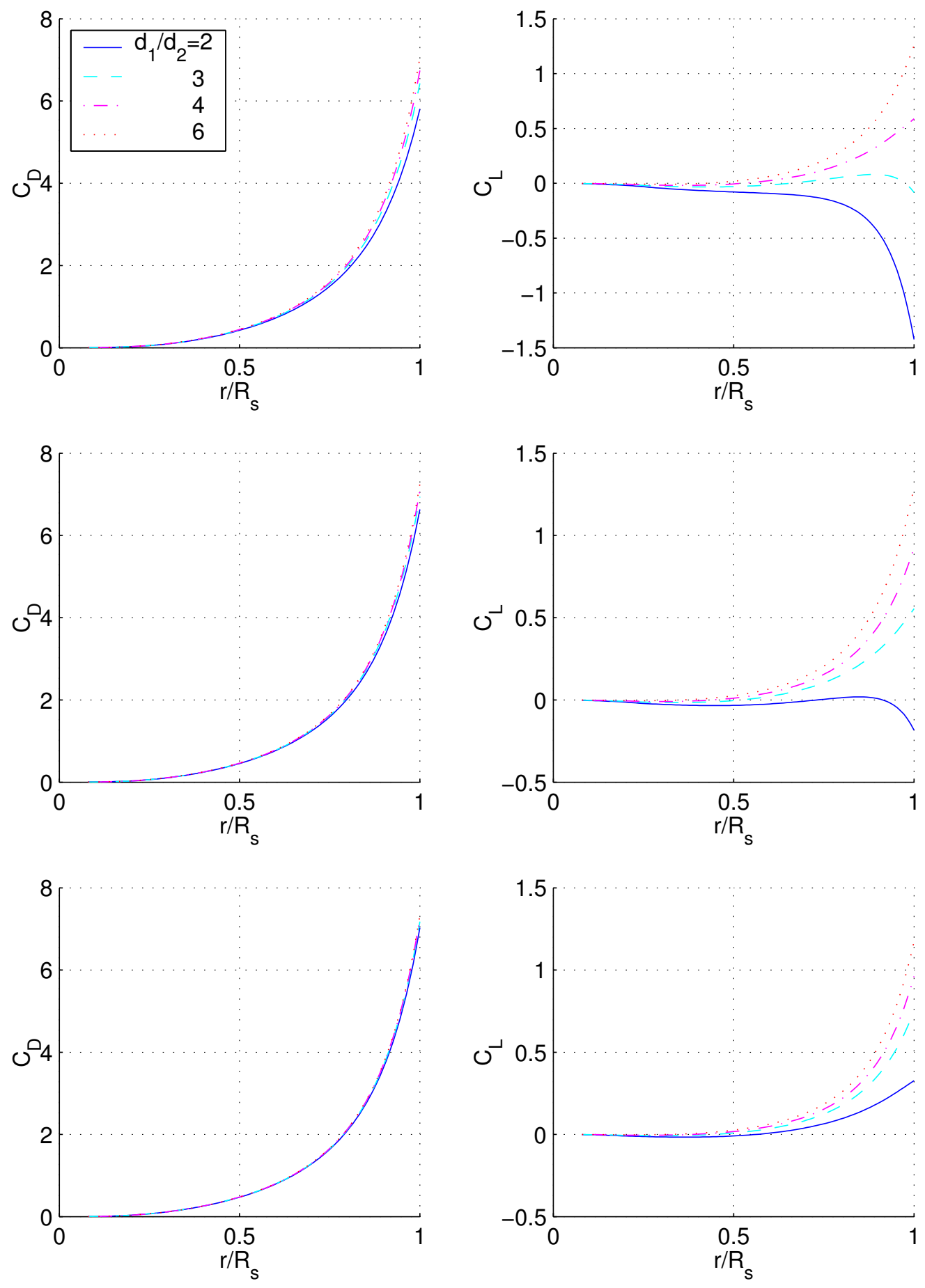

Figure 2.6: Theoretical secondary drag and lift coefficients in two dimensions, using the modified Newtonian profile without $x$-derivatives, for various $d_{1} / d_{2}$ ratios at $x / d_{1}=2.5$ (top), 5 (center), and 10 (bottom). 

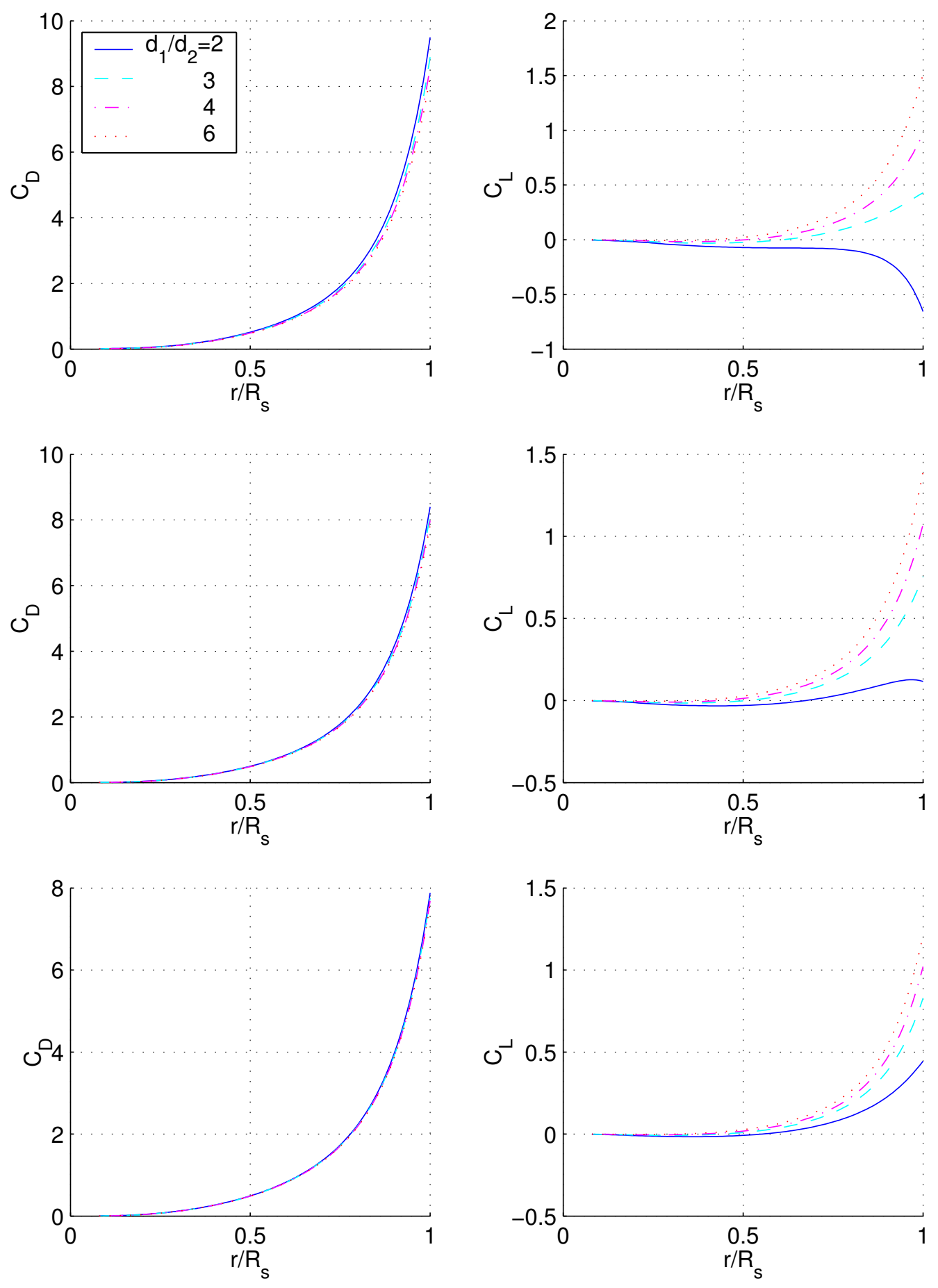

Figure 2.7: Theoretical secondary drag and lift coefficients in two dimensions, using the modified Newtonian profile with $x$-derivatives, at $x / d_{1}=2.5$ (top), 5 (center), and 10 (bottom). 


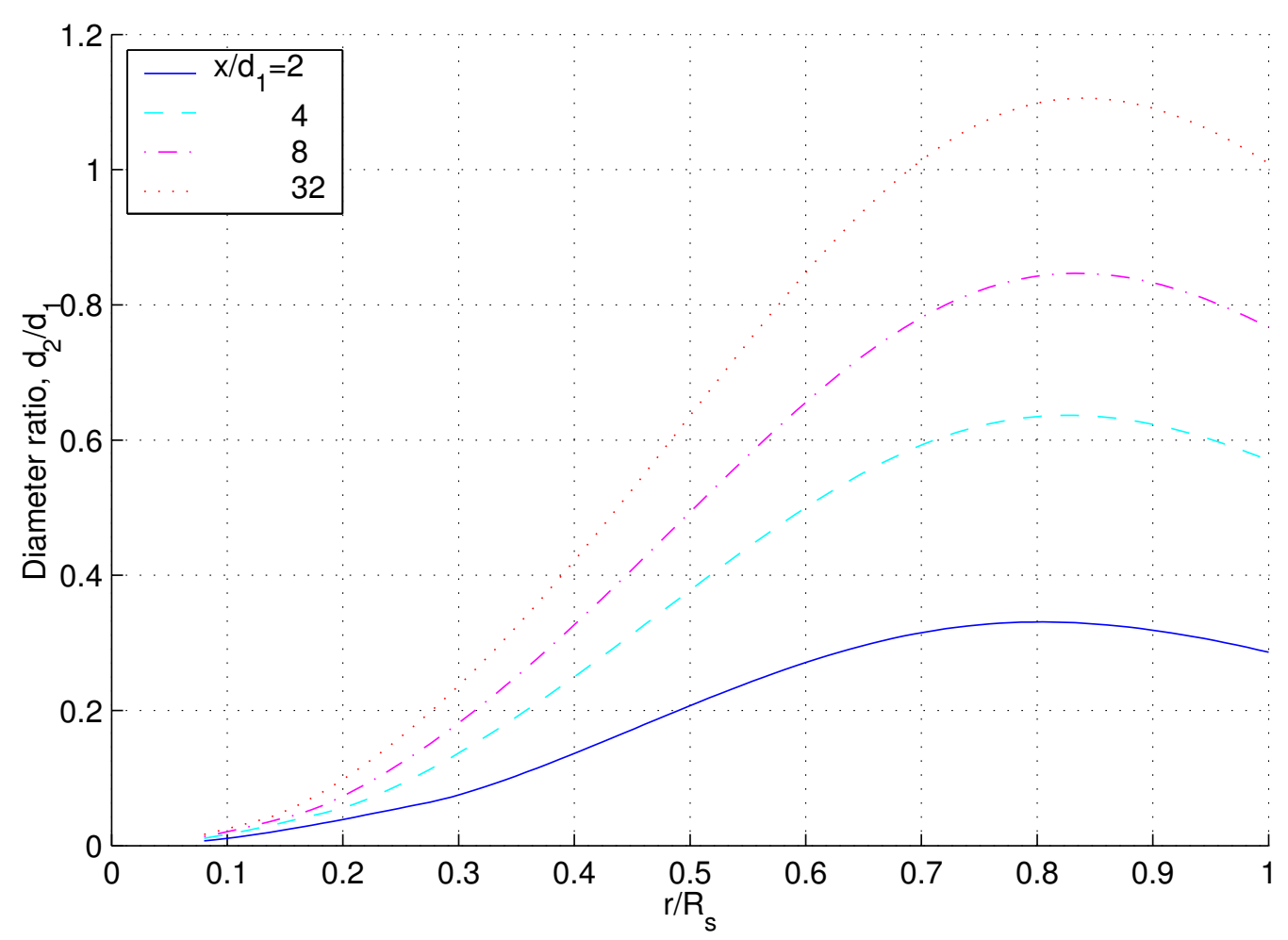

Figure 2.8: Diameter ratio in two dimensions at which a cross-over from a positive to a negative lift coefficient occurs

\subsection{Modeling in Three Dimensions}

\subsubsection{The Axisymmetric Blast Wave Analogy}

An axisymmetric blast wave is analagous to the flow produced by an axisymmetric body. The similarity variable in this case is

$$
\eta=\left(\frac{\rho_{\infty}}{E}\right)^{1 / 4} \frac{r}{\sqrt{t}} \longrightarrow\left(\frac{2}{A C_{D}}\right)^{1 / 4} \frac{r}{\sqrt{x}},
$$

where $\rho_{\infty}$ is again the ambient density and $E$ the energy released, per unit depth, in the analogous explosion. $A$ and $C_{D}$ are the projected frontal area and drag coefficient of the body, respectively. $E$ has been equated with the drag force on the body, given by $\frac{1}{2} C_{D} \rho_{\infty} V^{2} A$. We assume the body to be a sphere, so $A=\pi d_{1}^{2} / 4$. The drag coefficient is given a value of 0.88 , which is appropriate for a sphere in high Mach 
number flow.

The shock radius is then given by

$$
R_{s}=\eta_{0}\left(\frac{\pi d_{1}^{2} C_{D}}{8}\right)^{1 / 4} \sqrt{x}
$$

Here $\eta_{0}$ takes the values given in Table 2.2. The reader is again referred to Landau and Lifshitz (1989) for details.

\begin{tabular}{c|c|c|c}
$\gamma$ & 1.30 & 1.40 & 1.67 \\
\hline$\eta_{0}$ & 0.9317 & 1.0040 & 1.11554
\end{tabular}

Table 2.2: Values of $\eta_{0}$ in axisymmetric geometry for various values of $\gamma$

The nondimensional dependent variables are

$$
\begin{aligned}
& \hat{p}=2(\gamma+1) \frac{p}{\rho_{\infty}(r V / x)^{2}}, \\
& \hat{u}=(\gamma+1) \frac{u}{r V / x}, \\
& \hat{\rho}=\frac{\gamma-1}{\gamma+1} \frac{\rho}{\rho_{\infty}},
\end{aligned}
$$

where the constants have again been introduced to simplify the boundary conditions at $r=R_{s}$.

The equations of motion are

$$
\begin{aligned}
\rho \frac{\partial u}{\partial r}+u \frac{\partial \rho}{\partial r}+V \frac{\partial \rho}{\partial x}+\frac{\rho u}{r} & =0 \\
u \frac{\partial u}{\partial r}+V \frac{\partial u}{\partial x}+\frac{1}{\rho} \frac{\partial p}{\partial r} & =0 \\
\left(u \frac{\partial}{\partial r}+V \frac{\partial}{\partial x}\right) \log \frac{p}{\rho^{\gamma}} & =0
\end{aligned}
$$


and the resulting solution:

$$
\begin{aligned}
\left(\frac{\eta_{0}}{\eta}\right)^{2} & =u\left(\frac{2 \gamma \hat{u}-(\gamma+1)}{\gamma-1}\right)^{k_{1}}(\gamma+1-\gamma u) \\
\hat{\rho} & =\left(\frac{\gamma+1-2 \hat{u}}{\gamma-1}\right)^{k_{2}}\left(\frac{2 \gamma \hat{u}-(\gamma+1)}{\gamma-1}\right)^{k_{3}}(\gamma+1-\gamma u)^{k_{4}} \\
\frac{\hat{p}}{\hat{\rho}} & =\frac{\hat{u}^{2}(\gamma+1-2 \hat{u})}{2 \gamma \hat{u}-(\gamma+1)}
\end{aligned}
$$

where

$$
\begin{aligned}
k_{1} & =-\frac{\gamma-1}{\gamma} \\
k_{2} & =-\frac{2}{2-\gamma} \\
k_{3} & =\frac{1}{\gamma} \\
k_{4} & =\frac{2}{2-\gamma} .
\end{aligned}
$$

The dimensional forms of these variables, normalized by their values at the shock, are plotted in Figure 2.9 for $\gamma=1.4$. This value will again be assumed throughout this section.

The Mach number $M$ and flow angle $\delta$ may again be obtained:

$$
\begin{aligned}
M & =\left(2 \frac{\left(\frac{\gamma+1}{\eta}\right)^{2}\left(\frac{8}{\pi C_{D}}\right)^{\frac{1}{2}} \frac{x}{d_{1}}+\hat{u}^{2}}{\gamma(\gamma-1) \hat{p} / \hat{\rho}}\right)^{\frac{1}{2}} \\
\delta & =\arctan \left(\frac{1}{\gamma+1}\left(\frac{\pi C_{D}}{8}\right)^{1 / 4}\left(\frac{x}{d_{1}}\right)^{-1 / 2} \eta \hat{u}\right) .
\end{aligned}
$$

These are plotted in Figure 2.10 for various distances downstream. Note again that the Mach number at a given value of $\eta$ increases without bound as $x / d_{1} \rightarrow \infty$. 


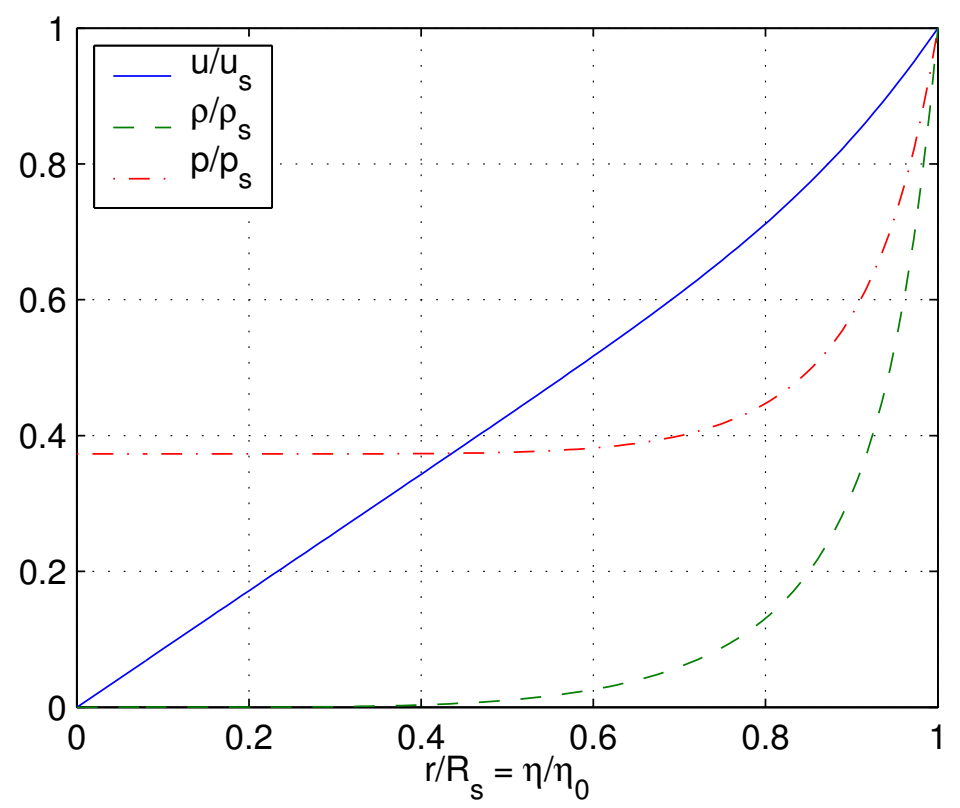

Figure 2.9: Variables in the axisymmetric blast wave analogy for $\gamma=1.4$

\subsubsection{Proximal Bodies in Three Dimensions}

\subsubsection{Pressure Distribution on the Secondary Body}

To estimate the forces acting on a second body placed in the shocked region given by the axisymmetric blast wave analogy, we proceed in a manner similar to the planar case. We specify this body also to be a sphere since, as in the planar case, the problem will become more tractable if the secondary body possesses rotational symmetry. We also assume that it is placed above and behind the primary sphere, so that the relevant lateral force coefficient is the lift coefficient, with a positive value indicating a repulsive force from the axis of symmetry of the primary body. Again, the secondary body is assumed to be stationary relative to the primary body.

The Newtonian-flow pressure distribution on a sphere in the uniform freestream outside the shocked region is

$$
C_{p}(\theta)= \begin{cases}2 \cos ^{2} \theta & \text { for } \theta \in[0, \pi / 2) \\ 0 & \text { for } \theta \in[\pi / 2, \pi)\end{cases}
$$



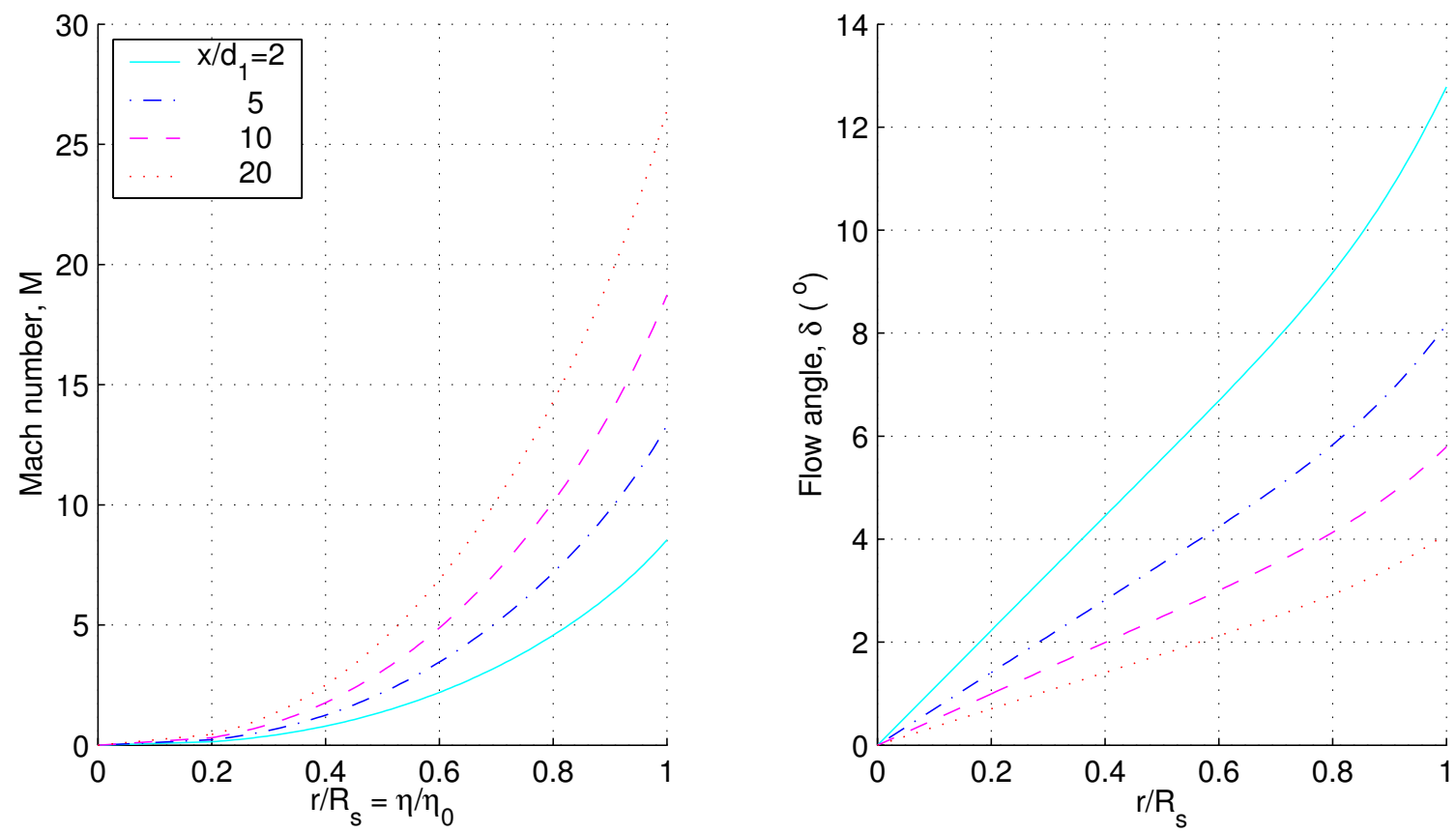

Figure 2.10: Mach number and flow angle profiles at various distances downstream in the axisymmetric blast wave analogy

where $\theta$ is the zenith angle measured from the stagnation point.

The modified Newtonian description gives $p(\theta)=\left(p_{\text {stag }}-p_{\infty}\right) \cos ^{2} \theta+p_{\infty}$ for $\theta \in[0, \pi / 2)$, where $p_{\text {stag }}$ may again be obtained from Equation 2.18. The corresponding normalized pressure distribution, $p(\theta) / p_{\text {stag }}$, is plotted in Figure 2.11 along with computed profiles for two different Mach numbers. These computed profiles were obtained from Amrita simulations using axisymmetric symmetry. As in the planar case, the computed stagnation point values differ slightly from the theoretical values. Again, we also try a Gaussian profile of the form $p(\theta)=p_{\text {stag }} \exp \left(-k \theta^{2}\right)$ to fit the region near $\theta=\pi / 2$ more closely. The value of $k$ is determined by a least-squares fit of the computed profiles ahead of the separation point. For $M=4,10$, and 20, values of $1.089,1.211$, and 1.191, respectively were obtained, so a representative value of $k=1.2$ was chosen.

The modified Newtonian profiles in Figure 2.11 show much better agreement with the computational profiles than in the case of a circular cylinder. The exponential 
tails of the Gaussian profile again do a poor job behind the separation point, so for lower Mach numbers the Newtonian profile seems preferable. For higher Mach numbers, however, at which the pressure jump behind the separation point becomes less significant, we expect the Gaussian distribution to perform well.
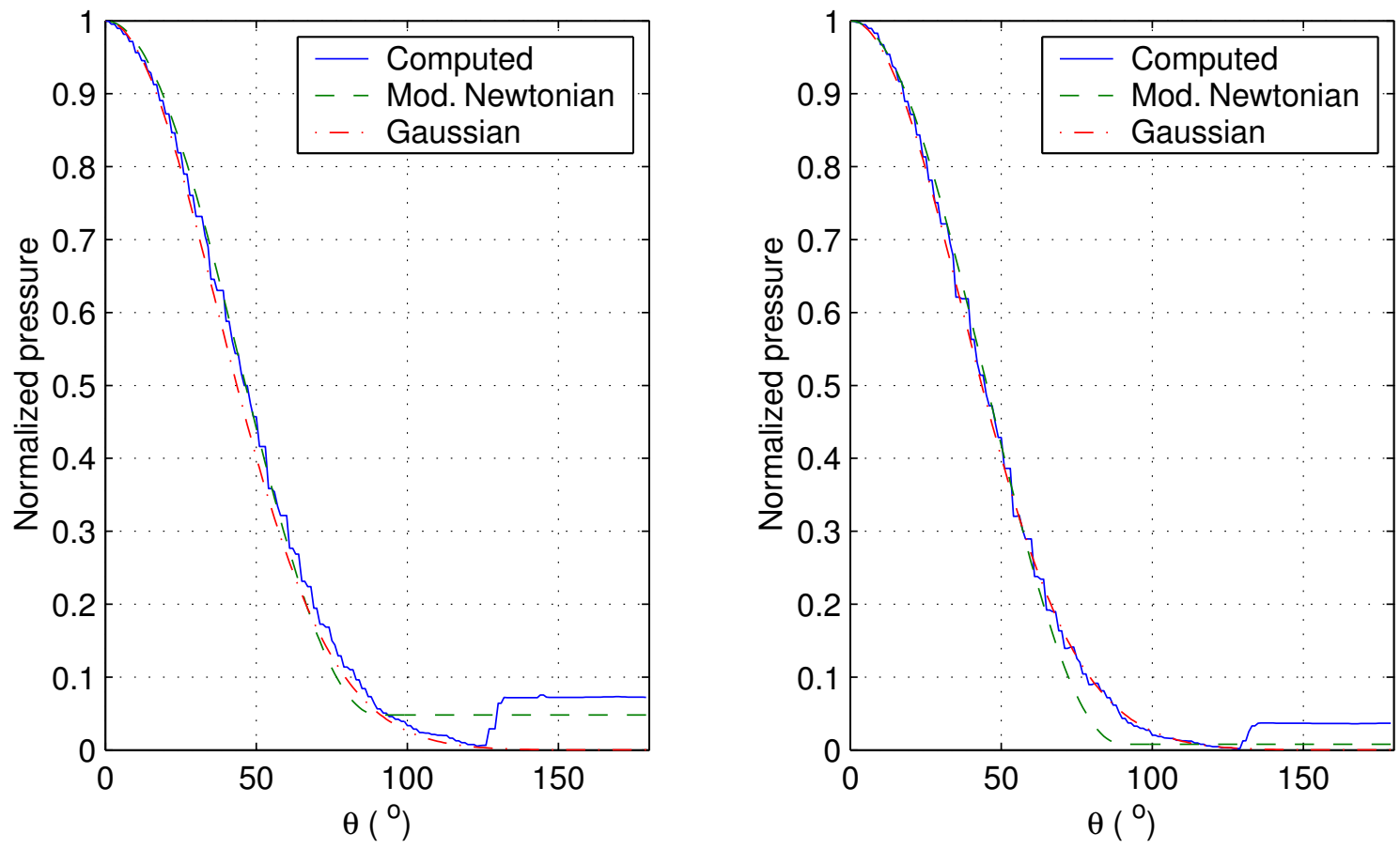

Figure 2.11: Theoretical and computed normalized pressure distributions on a sphere in a uniform freestream at $M_{\infty}=4$ (left) and $M_{\infty}=10$ (right)

We again define $p^{\prime}=p / \frac{1}{2} \rho_{\infty} V^{2}$ and write the modified Newtonian and Gaussian profiles as

$$
p^{\prime}(\theta)= \begin{cases}\left(p_{\text {stag }}^{\prime}-p_{\infty}^{\prime}\right) \cos ^{2} \theta+p_{\infty}^{\prime} & \text { for } \theta \in[0, \pi / 2) \\ p_{\infty}^{\prime} & \text { for } \theta \in[\pi / 2, \pi)\end{cases}
$$

and

$$
p^{\prime}(\theta)=p_{\text {stag }}^{\prime} \exp \left(-k \theta^{2}\right) \text { for } \theta \in[0, \pi)
$$


respectively.

We wish to use these reference profiles to model the pressure distribution on a sphere inside the shocked region. We thus redefine $p_{\text {stag }}^{\prime}$ as we did in the planar case to be the pressure obtained if the flow at a point in the blast wave solution is passed through a normal shock and subsequently decelerated isentropically to stagnation conditions, and $p_{1}^{\prime}$ replaces $p_{\infty}^{\prime}$ in Equation 2.49. The reference point for the blast wave conditions is again taken to be the center of the secondary body. In Figure 2.12, $p_{\text {stag }}^{\prime}$ as redefined here is plotted at various distances downstream. Noting that these profiles are qualitatively very similar to those in the planar case, we consider the same two deviations from the reference distributions as in the planar case, namely deflection of the flow angle and variation in stagnation pressure over the body.

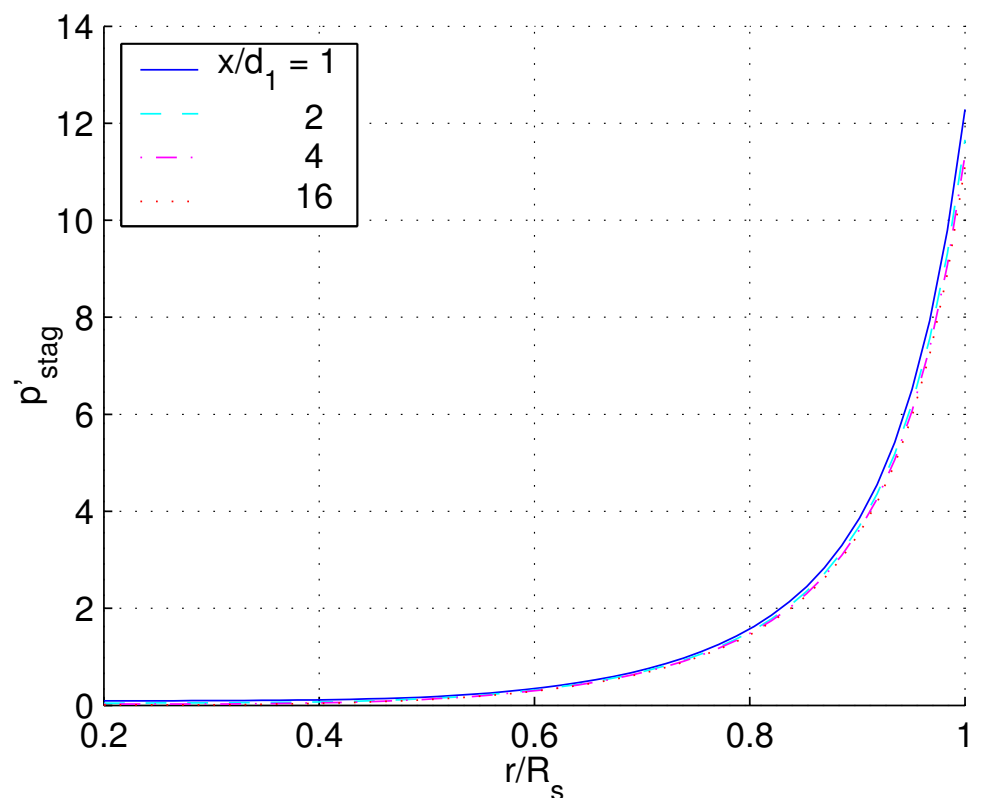

Figure 2.12: Pressure coefficient $p_{\text {stag }}^{\prime}$ at the stagnation point of a secondary body at various distances downstream

An additional complication is introduced in this case by the fact that isosurfaces in the blast wave solution are now axisymmetric rather than planar. To make the integration tractable, however, we treat the isosurfaces as locally flat in the region of the secondary body. This will be a reasonable approximation provided the displace- 
ment of the secondary body from the axis of symmetry of the primary body is large compared to the secondary body radius.

\subsubsection{Drag and Lift Coefficients}

Making use of Euler angles, we may write the drag and lift coefficients for the modified Newtonian case as

$$
\begin{aligned}
C_{D}= & \frac{1}{\pi} \int_{0}^{2 \pi} \int_{0}^{\pi / 2}\left[\left(p_{\text {stag }}^{\prime}-p_{1}^{\prime}\right)+\frac{1}{2} \frac{d_{2}}{d_{1}} \frac{\partial\left(p_{\text {stag }}^{\prime}-p_{1}^{\prime}\right)}{\partial\left(r / d_{1}\right)} \hat{y}\right. \\
& \left.-\frac{1}{2} \frac{d_{2}}{d_{1}} \frac{\partial\left(p_{\text {stag }}^{\prime}-p_{1}^{\prime}\right)}{\partial\left(x / d_{1}\right)} \hat{x}\right] \cos ^{2} \theta \hat{x} \sin \theta d \theta d \phi \\
& +\frac{1}{\pi} \int_{0}^{2 \pi} \int_{0}^{\pi}\left[p_{1}^{\prime}+\frac{1}{2} \frac{d_{2}}{d_{1}}\left(\frac{\partial p_{1}^{\prime}}{\partial\left(r / d_{1}\right)} \hat{y}-\frac{\partial p_{1}^{\prime}}{\partial\left(x / d_{1}\right)} \hat{x}\right)\right] \hat{x} \sin \theta d \theta d \phi \\
C_{L}= & -\frac{1}{\pi} \int_{0}^{2 \pi} \int_{0}^{\pi / 2}\left[\left(p_{\text {stag }}^{\prime}-p_{1}^{\prime}\right)+\frac{1}{2} \frac{d_{2}}{d_{1}} \frac{\partial\left(p_{\text {stag }}^{\prime}-p_{1}^{\prime}\right)}{\partial\left(r / d_{1}\right)} \hat{y}\right. \\
& \left.-\frac{1}{2} \frac{d_{2}}{d_{1}} \frac{\partial\left(p_{\text {stag }}^{\prime}-p_{1}^{\prime}\right)}{\partial\left(x / d_{1}\right)} \hat{x}\right] \cos ^{2} \theta \hat{y} \sin \theta d \theta d \phi \\
& -\frac{1}{\pi} \int_{0}^{2 \pi} \int_{0}^{\pi}\left[p_{1}^{\prime}+\frac{1}{2} \frac{d_{2}}{d_{1}}\left(\frac{\partial p_{1}^{\prime}}{\partial\left(r / d_{1}\right)} \hat{y}-\frac{\partial p_{1}^{\prime}}{\partial\left(x / d_{1}\right)} \hat{x}\right)\right] \hat{y} \sin \theta d \theta d \phi
\end{aligned}
$$

and for the Gaussian case:

$$
\begin{aligned}
C_{D}= & \frac{1}{\pi} \int_{0}^{2 \pi} \int_{0}^{\pi}\left(p_{\text {stag }}^{\prime}+\frac{1}{2} \frac{d_{2}}{d_{1}} \frac{\partial p_{\text {stag }}^{\prime}}{\partial\left(r / d_{1}\right)} \hat{y}\right. \\
& \left.-\frac{1}{2} \frac{d_{2}}{d_{1}} \frac{\partial p_{\text {stag }}^{\prime}}{\partial\left(r / d_{1}\right)} \hat{x}\right) \exp \left(-k \theta^{2}\right) \hat{x} \sin \theta d \theta d \phi \\
C_{L}= & -\frac{1}{\pi} \int_{0}^{2 \pi} \int_{0}^{\pi}\left(p_{\text {stag }}^{\prime}+\frac{1}{2} \frac{d_{2}}{d_{1}} \frac{\partial p_{\text {stag }}^{\prime}}{\partial\left(r / d_{1}\right)} \hat{y}\right. \\
& \left.-\frac{1}{2} \frac{d_{2}}{d_{1}} \frac{\partial p_{\text {stag }}^{\prime}}{\partial\left(x / d_{1}\right)} \hat{x}\right) \exp \left(-k \theta^{2}\right) \hat{y} \sin \theta d \theta d \phi,
\end{aligned}
$$


where

$$
\begin{aligned}
& \hat{y}=\cos \delta \sin \phi \sin \theta-\sin \delta \cos \theta \\
& \hat{x}=\sin \delta \sin \phi \sin \theta+\cos \delta \cos \theta
\end{aligned}
$$

For the modified Newtonian profile, the double integral may be evaluated to give

$$
\begin{aligned}
C_{D}= & \frac{1}{2} \cos \delta\left(p_{\text {stag }}^{\prime}-p_{1}^{\prime}\right)-\frac{1}{15} \frac{d_{2}}{d_{1}} \sin 2 \delta \frac{\partial\left(p_{\text {stag }}^{\prime}-p_{1}^{\prime}\right)}{\partial\left(r / d_{1}\right)} \\
& -\frac{1}{15} \frac{d_{2}}{d_{1}}(2+\cos 2 \delta) \frac{\partial\left(p_{\text {stag }}^{\prime}-p_{1}^{\prime}\right)}{\partial\left(x / d_{1}\right)}-\frac{2}{3} \frac{d_{2}}{d_{1}} \frac{\partial p_{1}^{\prime}}{\partial\left(x / d_{1}\right)} \\
C_{L}= & \frac{1}{2} \sin \delta\left(p_{\text {stag }}^{\prime}-p_{1}^{\prime}\right)-\frac{1}{15} \frac{d_{2}}{d_{1}} \sin 2 \delta \frac{\partial\left(p_{\text {stag }}^{\prime}-p_{1}^{\prime}\right)}{\partial\left(x / d_{1}\right)} \\
& -\frac{1}{15} \frac{d_{2}}{d_{1}}(2-\cos 2 \delta) \frac{\partial\left(p_{\text {stag }}^{\prime}-p_{1}^{\prime}\right)}{\partial\left(r / d_{1}\right)}-\frac{2}{3} \frac{d_{2}}{d_{1}} \frac{\partial p_{1}^{\prime}}{\partial\left(r / d_{1}\right)} .
\end{aligned}
$$

The integrals in the Gaussian case may also be evaluated, but lead to rather untidy expressions, and there is little point including them here. The full expressions may be found in Appendix A. The form of these expressions is similar to the equivalent Newtonian expressions, although the constants differ slightly, and the $p_{1}^{\prime}$ terms are absent.

As in the planar case, we may identify the dominant terms involving the diameter ratio in the drag and lift equations. For the Newtonian case, these give

$$
\begin{aligned}
C_{D} & \approx \frac{1}{2} p_{\text {stag }}^{\prime}-\frac{1}{5} \frac{d_{2}}{d_{1}} \frac{\partial p_{\text {stag }}^{\prime}}{\partial\left(x / d_{1}\right)} \\
C_{L} & \approx \frac{1}{2} p_{\text {stag }}^{\prime} \sin \delta-\frac{1}{15} \frac{d_{2}}{d_{1}} \frac{\partial p_{\text {stag }}^{\prime}}{\partial\left(r / d_{1}\right)}
\end{aligned}
$$

These approximate expressions are similar in form to the corresponding planar expressions, and the same comments apply. In particular, we see again that the lift expression has two terms of opposite sign, only the second of which has a dependence 
on body size. Thus we again predict a qualitative change in lift behaviour as the body size is varied. This is indeed seen in Figures 2.13 and 2.14, in which the full formulae for the modified Newtonian lift and drag coefficients are plotted. The profiles in Figure 2.14 include the $x$-derivative terms, whereas those in Figure 2.13 do not. While the effect of body size on the lift profiles is similar to that in the planar case, the downstream displacement effect does not seem to carry over - while the magnitude of the lift values decreases as $x / d_{1}$ is increased, there is very little qualitative change. This difference is probably due to the $\partial p_{s t a g}^{\prime} / \partial\left(r / d_{1}\right)$ term in Equation 2.34 decaying more rapidly than that in Equation 2.60, as the shock radius grows more rapidly in the planar case. The drag coefficient profile is seen to change very little with body size. In those profiles in which the $x$-derivative terms are absent, the effect of downstream displacement is also very small, although if the $x$-derivatives are included we see the drag values drop gradually as $x / d_{1}$ is increased.

As in the two-dimensional case, we may wish to ask if there is a critical body-size ratio above or below which the lift experienced by the secondary body is exclusively of one sign within the shocked region. We thus set Equation 2.58 to zero and solve for $d_{2} / d_{1}$. The resulting curves, as functions of $r / R_{s}$, are shown in Figure 2.15 for various distances downstream. These may be read in the same manner as in the two-dimensional case: at a given distance downstream, a secondary body for which the diameter ratio lies above the curve will experience a negative lift coefficient, and thus an attractive lateral force, while one lying below will experience a positive lift coefficient. In contrast to the two-dimensional case, however, there does appear to be a value of $d_{2} / d_{1}$ above which the lift coefficient will be exclusively negative throughout the shocked region. This critical value is at $d_{2} / d_{1} \approx 0.165$, i.e., for a primary body approximately six times the diameter of the secondary body. A secondary body with a diameter larger than this value, if placed within the shocked region with the same initial velocity as the primary body, is certain to be entrained within the shocked 

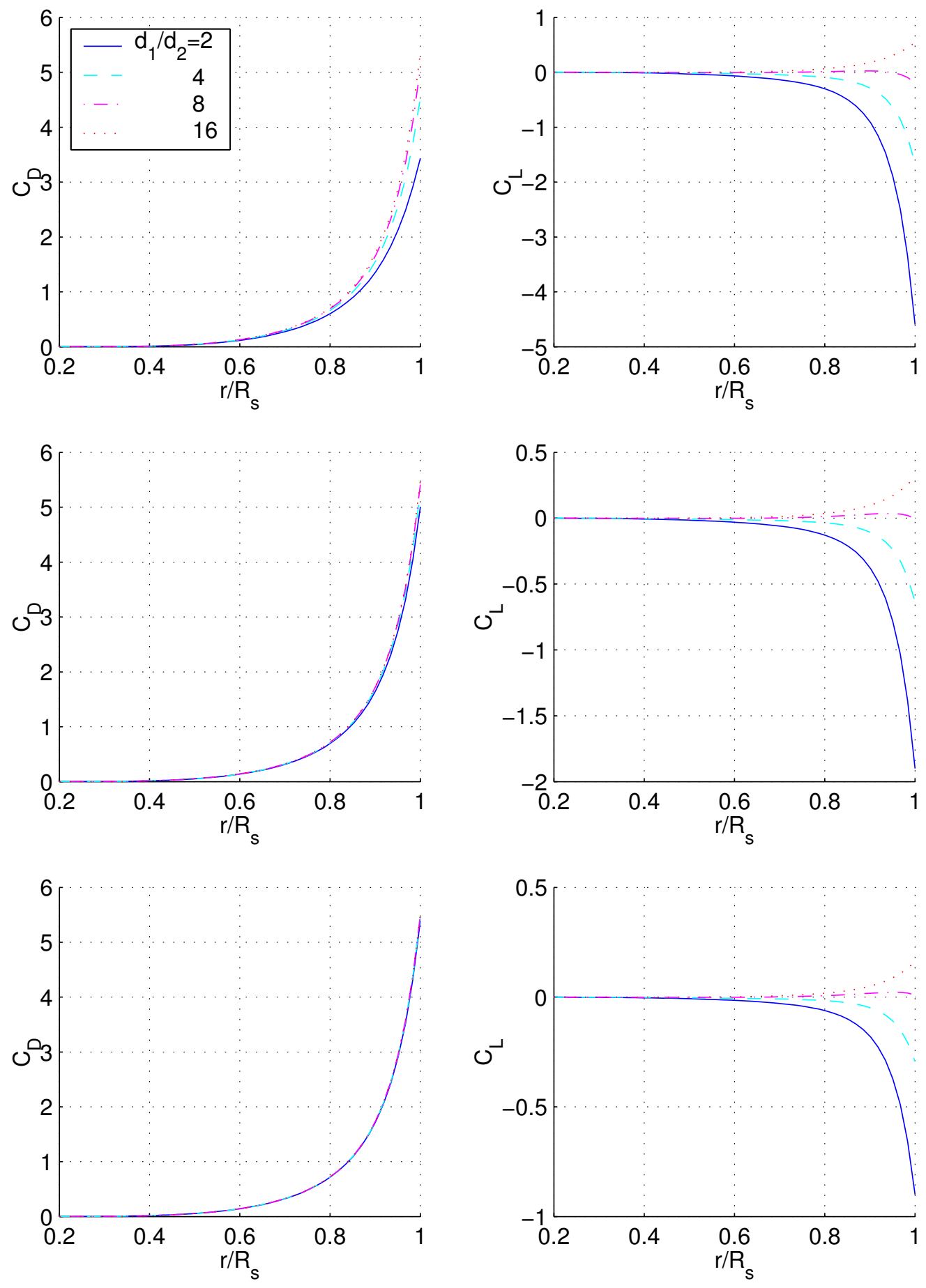

Figure 2.13: Theoretical secondary drag and lift coefficients in three dimensions, using the modified Newtonian distribution without $x$-derivatives, for various $d_{1} / d_{2}$ ratios at $x / d_{1}=2$ (top), 8 (center), and 32 (bottom). 

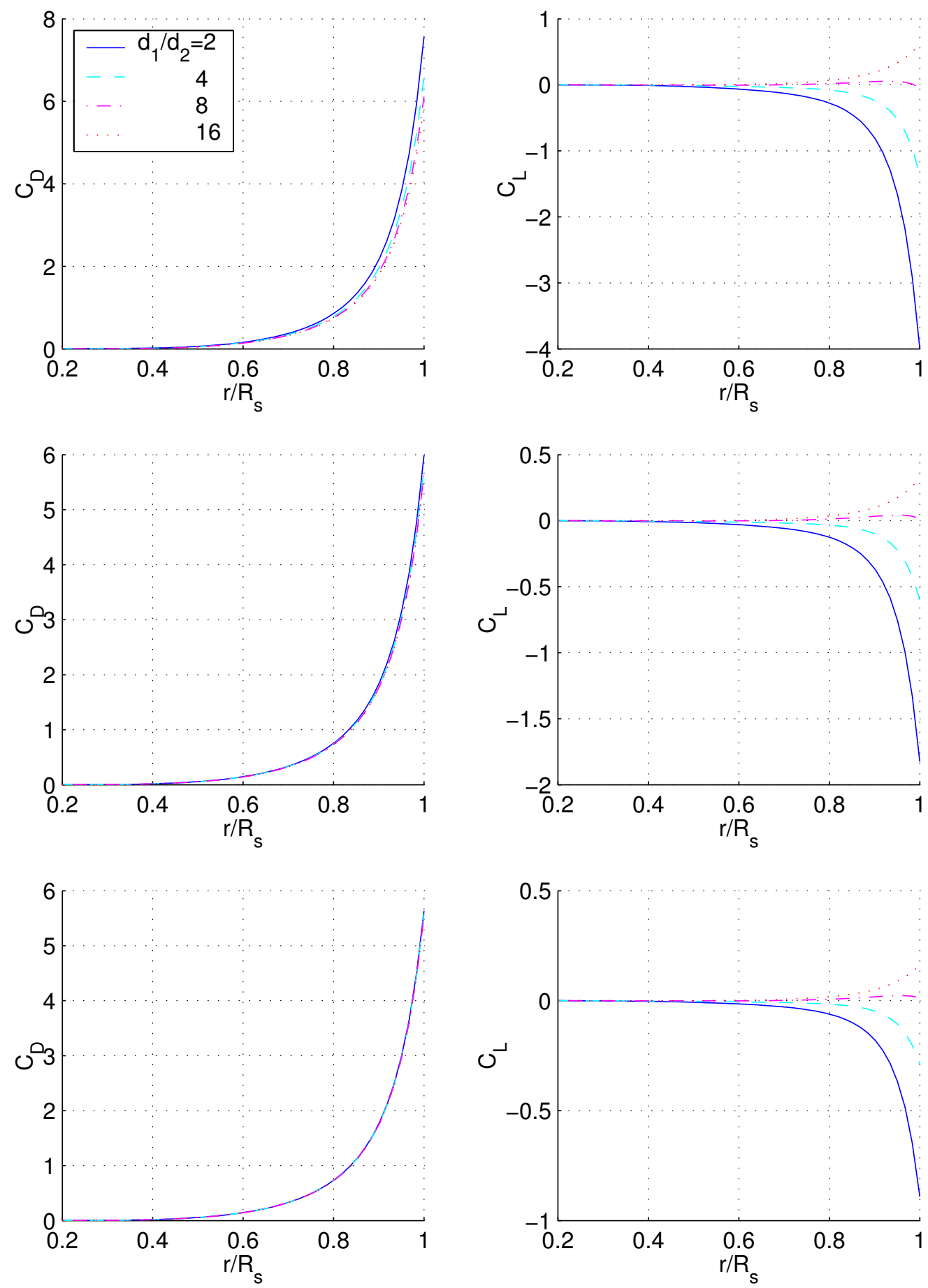

Figure 2.14: Theoretical secondary drag and lift coefficients in three dimensions, using the modified Newtonian distribution including $x$-derivatives, for various $d_{1} / d_{2}$ ratios at $x / d_{1}=2$ (top), 8 (center), and 32 (bottom). 
region, whereas smaller bodies have the possibility of being ejected.

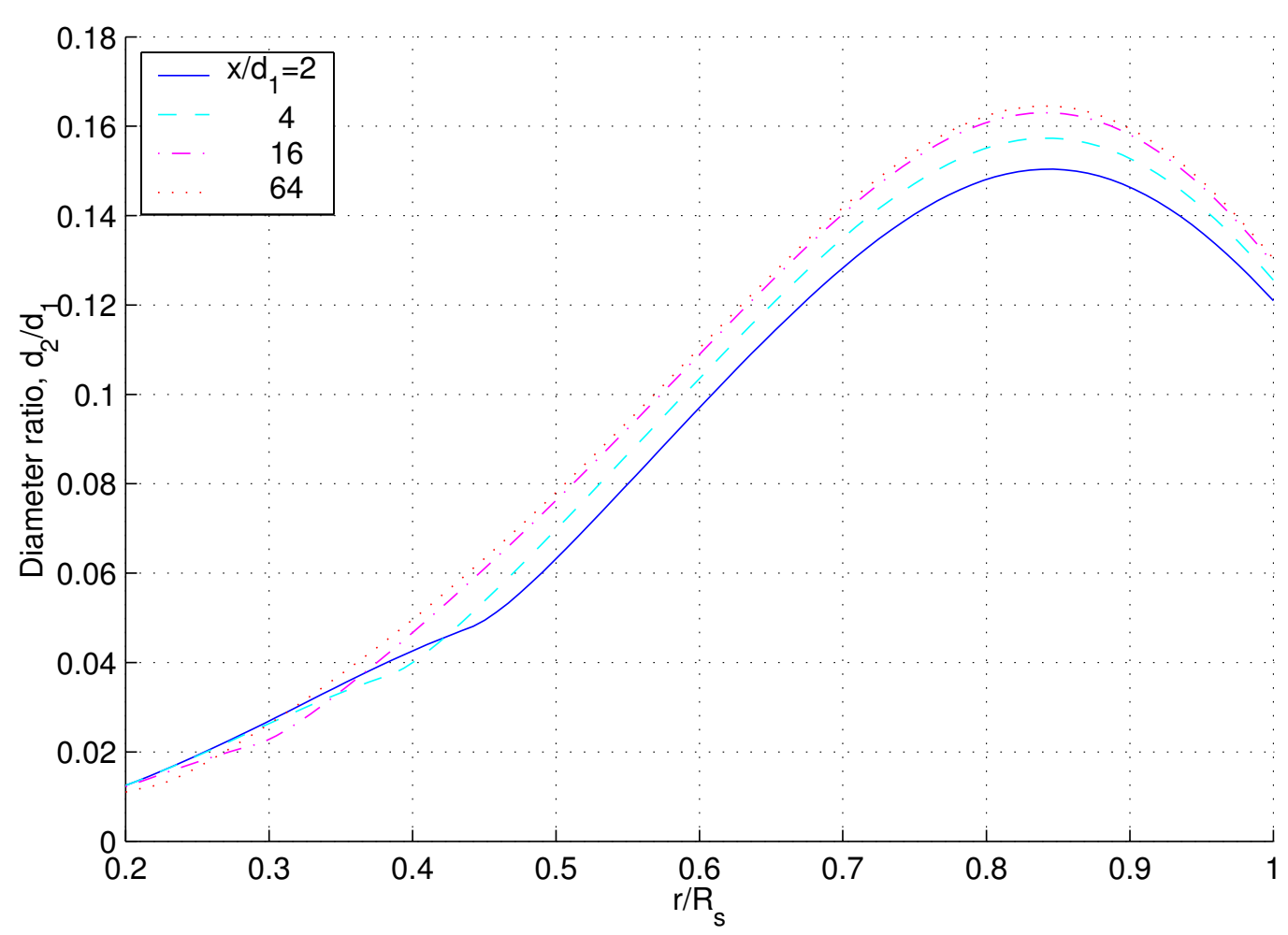

Figure 2.15: Diameter ratio in three dimensions at which a cross-over from a positive to a negative lift coefficient occurs

The effect that we have noted of body size on the secondary lift coefficient could have important consequences in situations such as the distribution of fragments in the crater field produced by the atmospheric breakup of a meteoritic body. Immediately after the fragmentation event, a single bow shock will encompass the collection of fragments. As the fragments separate, this shock will grow until the bodies are sufficiently far apart that they develop individual bow shocks. During the initial main shock phase, this body-size effect could lead to smaller fragments being ejected away from the collection, whereas larger fragments would be more likely to be entrained. At later times, one could imagine a large fragment entraining many smaller fragments behind it. This could lead to groupings of craters within the main field and would be similar to the effect of secondary fragmentation. This latter possibility will be discussed further in Chapter 7. 


\section{Chapter 3}

\section{Computational Modeling}

\subsection{Introduction}

Computational modeling of the proximal body problem has been carried out in both two and three dimensions. In order to provide direct comparisons with the analytical methodology developed in the previous chapter, simulated body geometries were limited to circular cylinders in the two-dimensional case and spheres in the

three-dimensional case. Conceivably, however, arbitrary body geometries could be simulated with the available software. In this chapter we will outline the details of the computational modeling a selection of the results will be presented; in the following chapter the complete results will be compared with those obtained using the blast wave methodology.

\subsection{Two-Dimensional Computations}

\subsubsection{Introduction}

Two-dimensional numerical simulations were performed using two different softwares. The principal software used was AMROC (Adaptive Mesh Refinement in Objectoriented $\mathrm{C}++$ ), developed by R. Deiterding (2003). AMROC is a mesh adaption framework specifically designed for the solution of hyperbolic fluid flow problems 
on distributed memory machines. Implementation of the ghost fluid method allows the integration of complex, time-dependent boundaries into simulations. AMROC computations were run on the CACR (Center for Advanced Computing Research) machine ASAP at the California Institute of Technology. ASAP is a Pentium-II based parallel cluster with a Gigabit network running under Linux. A typical simulation, in which the two-dimensional Euler equations were solved for an ideal gas, used between 6 and 18 nodes. Initially a hybrid Roe-HLLE scheme with Godunov dimensional splitting was used, but this was later changed to the Van-Leer flux vector-splitting scheme to maintain consistency with the three-dimensional computations. All results presented here were obtained with the Van-Leer scheme.

The second software used was Amrita, due to Quirk (1998). Amrita was not available on the parallel cluster, so computations were limited to a single Pentium-IV machine. This limited the size of computations that could be performed, so the results obtained using Amrita were mainly used as a consistency check for the AMROC results. The Amrita simulations also solved the two-dimensional Euler equations, using the HLLE scheme with Kappa-MUSCL reconstruction and Godunov dimensional splitting.

In all computations, unless otherwise specified, the ratio of specific heats is $\gamma=1.4$.

\subsubsection{Details of Computations}

In all two-dimensional simulations, both bodies were circular cylinders. The parameters that were varied were the downstream and lateral displacements of the secondary body, the freestream Mach number, and the ratio of radii of the two bodies (for convenience, in this chapter we will use the radius ratio rather than the diameter ratio of the previous chapter - the two are, of course, interchangeable). The runs were grouped according to the downstream displacement, Mach number, and radius ratio, and for each combination of these, a series of simulations was performed in which 
the lateral position of the secondary body was varied incrementally from immediately behind the primary body to outside the primary bow shock. The lateral displacement was in the upwards direction, so a positive lift coefficient indicates a repulsive force from the plane of symmetry of the primary body.

Details of the AMROC computations are given in Table 3.1. The distance downstream value is the center-to-center displacement of the secondary body relative to the primary body, normalized by the radius of the primary body. The additional refinement entry indicates the refinement factor for each level of additional refinement over the base grid. Thus, for all simulations, two additional levels of refinement were used, each with a refinement factor of 2. The number of CPU hours in each case is a typical value, as the actual number could vary from computation to computation, especially if a different number of nodes was used. The number of timesteps could also vary slightly between computations in a given series.

\begin{tabular}{|l|c|c|c|c|}
\hline $\begin{array}{c}\text { Distance downsteam } \\
\text { (primary body radii) }\end{array}$ & 4 & 4 & 8 & 8 \\
\hline Mach number, $M$ & 10 & 50 & 10 & 50 \\
\hline Body radius ratio $\left(r_{1} / r_{2}\right)$ & 2,6 & 2,6 & 2,6 & 2,6 \\
\hline Base grid & $200 \times 200$ & $200 \times 200$ & $320 \times 280$ & $320 \times 280$ \\
\hline Additional refinement & 2,2 & 2,2 & 2,2 & 2,2 \\
\hline Physical domain & $2.5 \times 2.5$ & $2.5 \times 2.5$ & $4.0 \times 3.5$ & $4.0 \times 3.5$ \\
\hline Primary body radius & 0.24 & 0.24 & 0.24 & 0.24 \\
\hline CFL number & 0.9 & 0.7 & 0.9 & 0.7 \\
\hline Number of timesteps & 3100 & 4250 & 3100 & 3600 \\
\hline Last time & 3.0 & 0.7 & 3.0 & 0.6 \\
\hline $\begin{array}{l}\text { Computational overhead } \\
\text { (nodes/CPU hours) }\end{array}$ & $12 / 35$ & $12 / 42$ & $12 / 60$ & $12 / 65$ \\
\hline
\end{tabular}

Table 3.1: Details of two-dimensional AMROC computations.

For each run, the lift and drag values on each body were calculated every 10 timesteps by a numerical integration of the pressure with the appropriate component of the surface normal vector over the body. The mean lift and drag values were then calculated over the final $30 \%$ of the flow time. 
Amrita computations were carried out on a $264 \times 264$ coarse grid with one level of additional refinement, with a refinement factor of 2. The physical domain in Amrita simulations corresponds to the mesh dimensions, and the radius of the primary body was 24. These simulations thus had approximately half the resolution of the AMROC simulations. Each computation consisted of 5000 timesteps with a CFL number of 0.6 to a final time of around $t=200$. The lift and drag values were averaged over the last $10 \%$ of this time period. A single computation took approximately 8 hours on a Pentium IV machine running under Linux.

\subsubsection{Results}

The flow development during a typical AMROC simulation is shown in Figure 3.1. The visualisations are computational schlieren images overlaid on the refinement level sets and are taken at four time instants in the flow development. The first three images are taken during the establishment of the flow, while the lower right image shows the steady flow solution. The flow is established by ramping up the velocity at the inlet on the left boundary at constant pressure and density, resulting in the observed shock system. The plot below shows the lift and drag coefficients experienced by the secondary body, as well as the drag coefficient for the primary body, during this startup process. The time instants corresponding to the first three of the images are indicated by the dashed vertical lines. The Mach number in this case was 10, the downstream and lateral displacements were both 4 primary body radii (centerto-center), and the ratio of body radii was 2 .

Figures 3.2 and 3.3 show, for two combinations of downstream displacement and radius ratio, the drag and lift coefficients of the secondary body as functions of lateral displacement - in each case the configuration is shown in the schlieren image above. The coefficients are plotted for both $M=10$ and $M=50$. In Figure 3.2 the ratio of radii is 6 , and the downstream displacement is 4 primary body radii. Error bars 

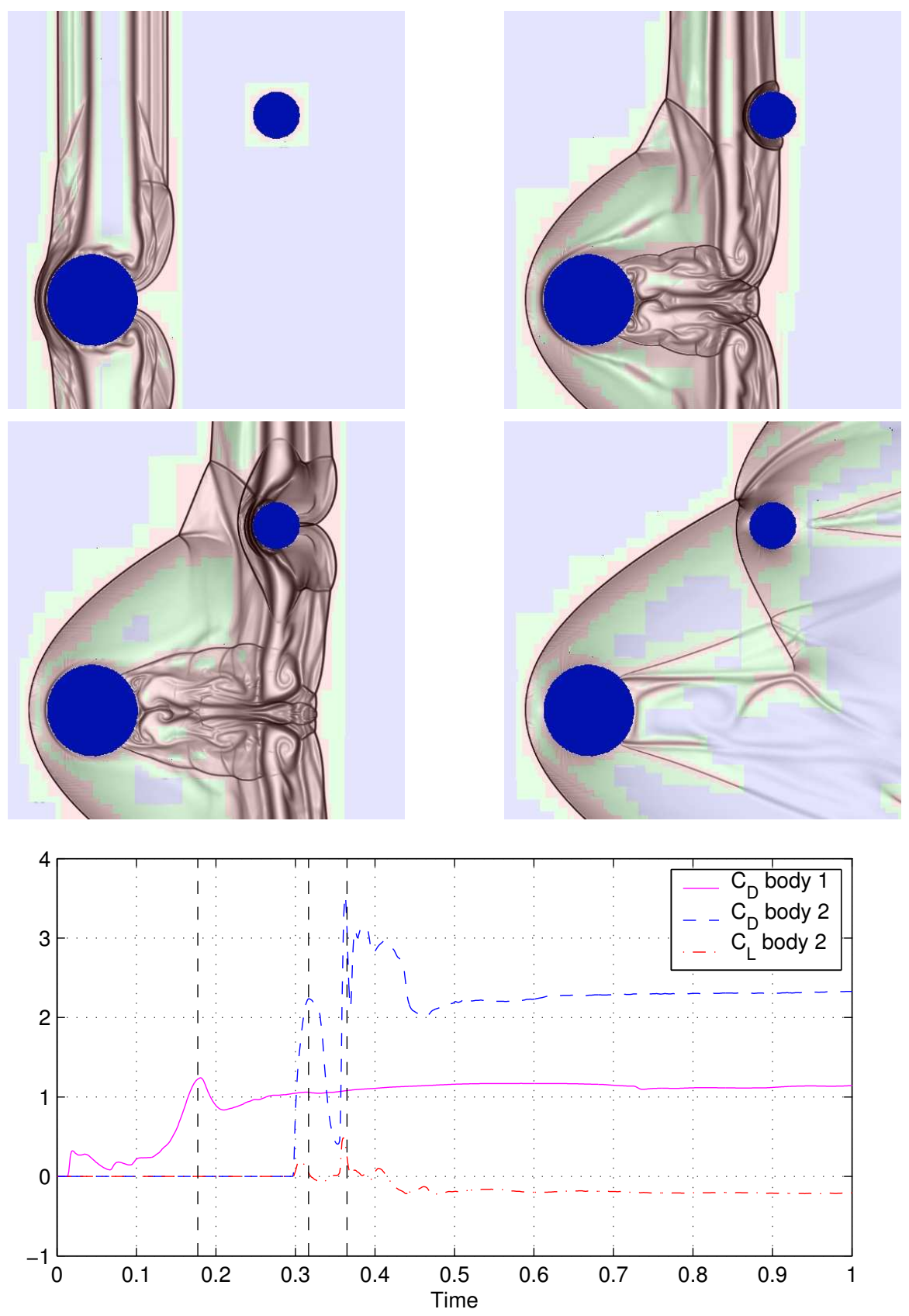

Figure 3.1: Flow development in a typical two-dimensional computation. Computational schlieren images overlaid on refinement level sets are shown at, clockwise from top left, $t=0.177,0.317,3.0,0.365$. The drag and lift coefficient profiles are plotted below with the three earliest times indicated. 
are included for those points in which the standard deviation of the drag value is greater than $5 \%$ of the mean value over the averaging time. As may be seen, this occurs exclusively near the wake region, where we would expect some unsteadiness to be present. Near $y / R=1$, the unsteadiness is caused by interactions between the secondary body and the separation shock from the primary body. As there are subsonic regions in the wake, information can propagate upstream from the secondary body, and this causes oscillations in the position of the separation shock. These in turn produce unsteadiness in the flow around the secondary body. A small hump in the drag profile is also typically seen in this region, as the interaction of the separation shock with the secondary bow shock produces locally high pressure.

As $y / R$ is increased further, we see that the $M=10$ and $M=50$ profiles are almost identical for both lift and drag. For this configuration, both increase monotonically with increasing $y / R$ until maximum values are reached at $y / R \approx 4.5$, at which point the primary shock is impinging near the leading point of the secondary body. The $M=50$ profiles do climb slightly more steeply, as the primary shock radius is smaller than in the $M=10$ case. As the secondary body clears the primary shock, the drag and lift coefficients revert to their freestream values of $C_{D} \approx 1.2$ and $C_{L}=0$.

In Figure 3.3, the ratio of radii is 2 and the downstream displacement is 8 primary body radii. Again, unsteadiness is observed near the wake region and is seen to persist to larger lateral displacements in the $M=10$ case. This is a result of the separation shock lying closer to the primary body's plane of symmetry at higher Mach numbers. Once outside the wake/separation shock region, the drag profiles conform to one another more closely, but again the $M=50$ profile rises more steeply due to the smaller primary shock radius. The lift profiles also conform over a small region, but then show some qualitative differences: the $M=10$ profile rises slightly before the shock impingement point (here at $y / R \approx 6$ ), whereas the $M=50$ values continue to decrease until this point is reached. Note also the contrast between the lift profiles 

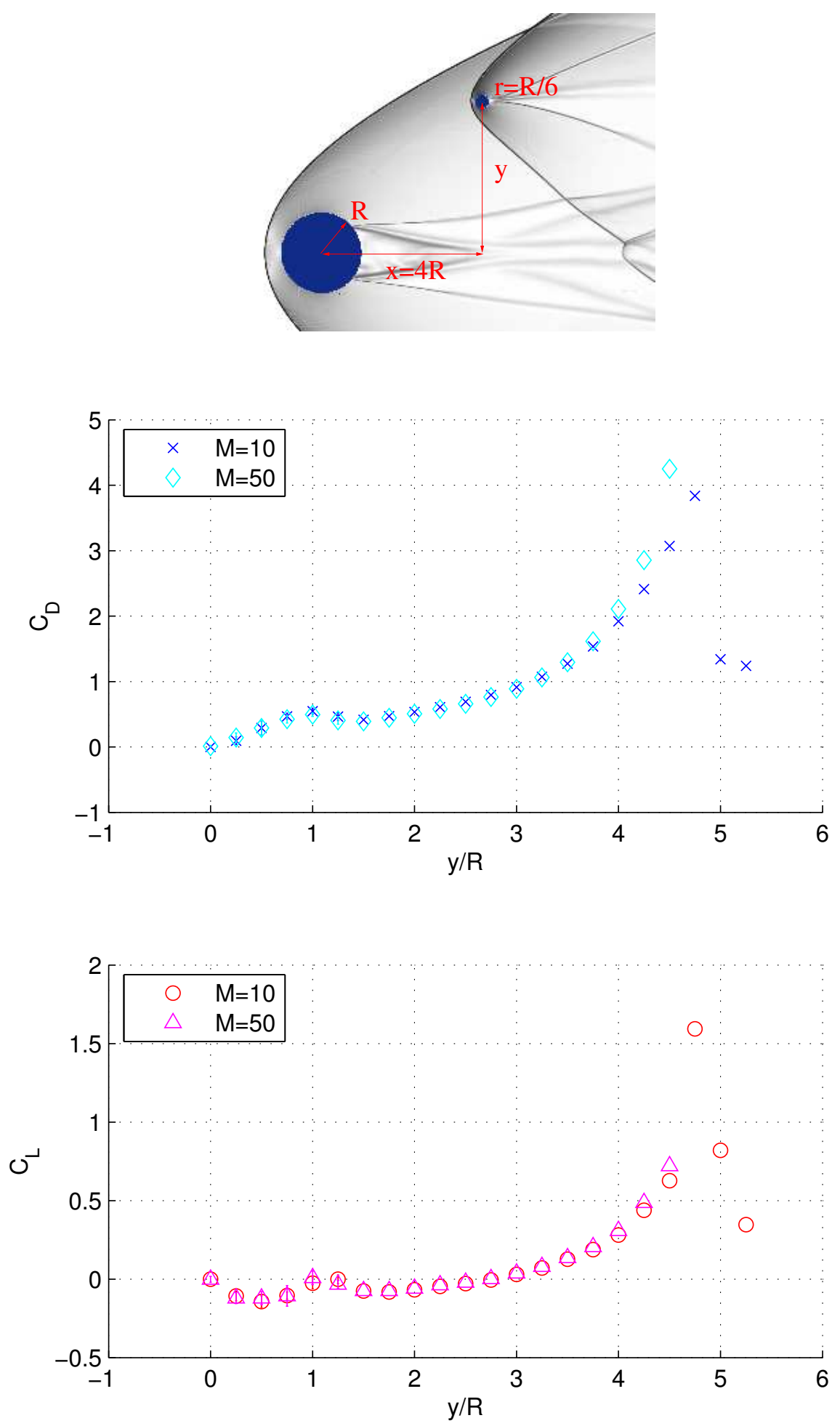

Figure 3.2: Drag and lift coefficents as functions of lateral displacement for cylinders in a configuration as shown above. The freestream Mach number in the schlieren image is $M=10$. 

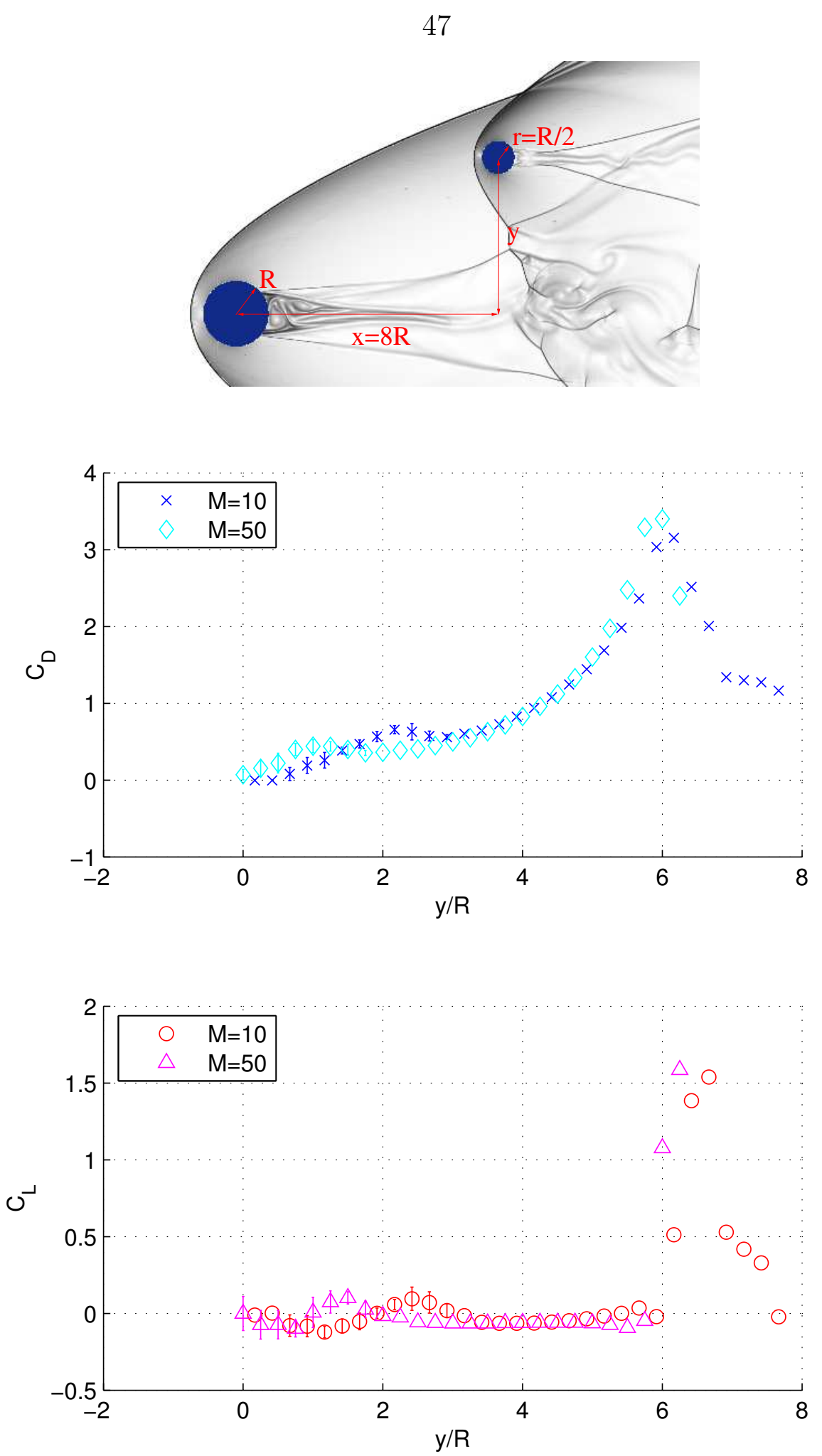

Figure 3.3: Drag and lift coefficents as functions of lateral displacement for cylinders in a configuration as shown above. The freestream Mach number in the schlieren image is $M=50$. 
in Figures 3.2 and 3.3. In the former, with a body radius ratio of 6 , the lift is mainly positive in the region between the separation and the bow shock, whereas in the latter, with a radius ratio of 2 , the tendency is for the lift coefficient to remain negative. This observation conforms to the predictions of Chapter 2 regarding the effect of body size on lift. Once the shock impingement point is reached, both lift profiles rise sharply to positive values, as was predicted in Chapter 1. This was explained crudely by the observation that, in this configuration, whereas the upper side of the body will experience singly-shocked flow, the lower side of the body will experience doubly-shocked, and thus higher pressure, flow.

The close agreement between the profiles for the two Mach numbers in Figure 3.2 is a demonstration of the Mach number independence principle, which states that as the Mach number is increased, the flow becomes increasingly independent of further changes in Mach number. This principle holds well in the near-field, but becomes more approximate in the far-field - hence the larger discrepancies seen in the profiles in Figure 3.3. The primary shock shapes, for example, must diverge somewhat in the far-field, as each must tend to the Mach angle for that particular Mach number.

For validation purposes, the Amrita software system was also used to simulate this problem for a particular choice of parameters, namely a Mach number of 10, a downstream displacement of 4 primary body radii, and a radius ratio of 2. Figure 3.4 shows a comparison of the lift and drag coefficients obtained with the two softwares. As may be seen, although agreement is reasonably good over much of the domain, there are some discrepancies, particularly as the primary bow shock begins to impinge on the secondary body. This is not entirely unexpected, however. We have already noted that the AMROC simulations have approximately twice the resolution of those performed with Amrita, and this extra resolution will become most notable in the vicinity of high-gradient flow features, such as shocks. In particular, as the primary bow shock becomes better resolved, the effective shock position could change slightly. 
To provide a more fair comparison then, a single Amrita simulation with two levels of additional refinement over the base grid was also carried out, giving approximately the same resolution as the Amroc simulations. The resulting drag and lift coefficients are shown in Table 3.2 and are compared with AMROC values computed at the same lateral displacement. The more refined Amrita coefficients agree very closely with the AMROC values obtained with two additional levels.

Table 3.2 also contains the results of a refinement study carried out with AMROC. The downstream and lateral displacements of the secondary body are both 4 primary body radii, the ratio of body radii is 2, and the Mach number is 10 (this choice of parameters is shown in Figure 3.1 for two levels of additional refinement). These values were chosen because in this configuration some of the largest discrepancies were seen between the Amrita and AMROC results, both in lift and drag coefficients (see Figure 3.4). We therefore might expect this to be a worst-case scenario with respect to the effect of refinement on the lift and drag values obtained. Up to three additional levels of refinement over the base grid were used, each with a refinement factor of 2. The lift and drag do appear to be converging, but the lift especially seems to be quite sensitive to changes in refinement, even between the two highest levels.

\begin{tabular}{|c|c|c|c|c|}
\hline $\begin{array}{c}\text { Additional. } \\
\text { levels }\end{array}$ & $C_{D}$ & $\Delta C_{D}$ & $C_{L}$ & $\Delta C_{L}$ \\
\hline $\begin{array}{c}\text { Amroc } \\
0\end{array}$ & 2.051 & & -0.051 & \\
1 & 2.117 & 0.066 & -0.128 & -0.077 \\
2 & 2.320 & 0.203 & -0.228 & -0.100 \\
3 & 2.346 & 0.026 & -0.183 & 0.045 \\
Amrita & & & & \\
1 & 2.076 & & -0.120 & \\
2 & 2.288 & 0.212 & -0.227 & -0.107 \\
\hline
\end{tabular}

Table 3.2: $C_{D}$ and $C_{L}$ values for two-dimensional refinement study and comparison with Amrita. $\Delta$ indicates the difference between the values at current and previous levels of refinement. 

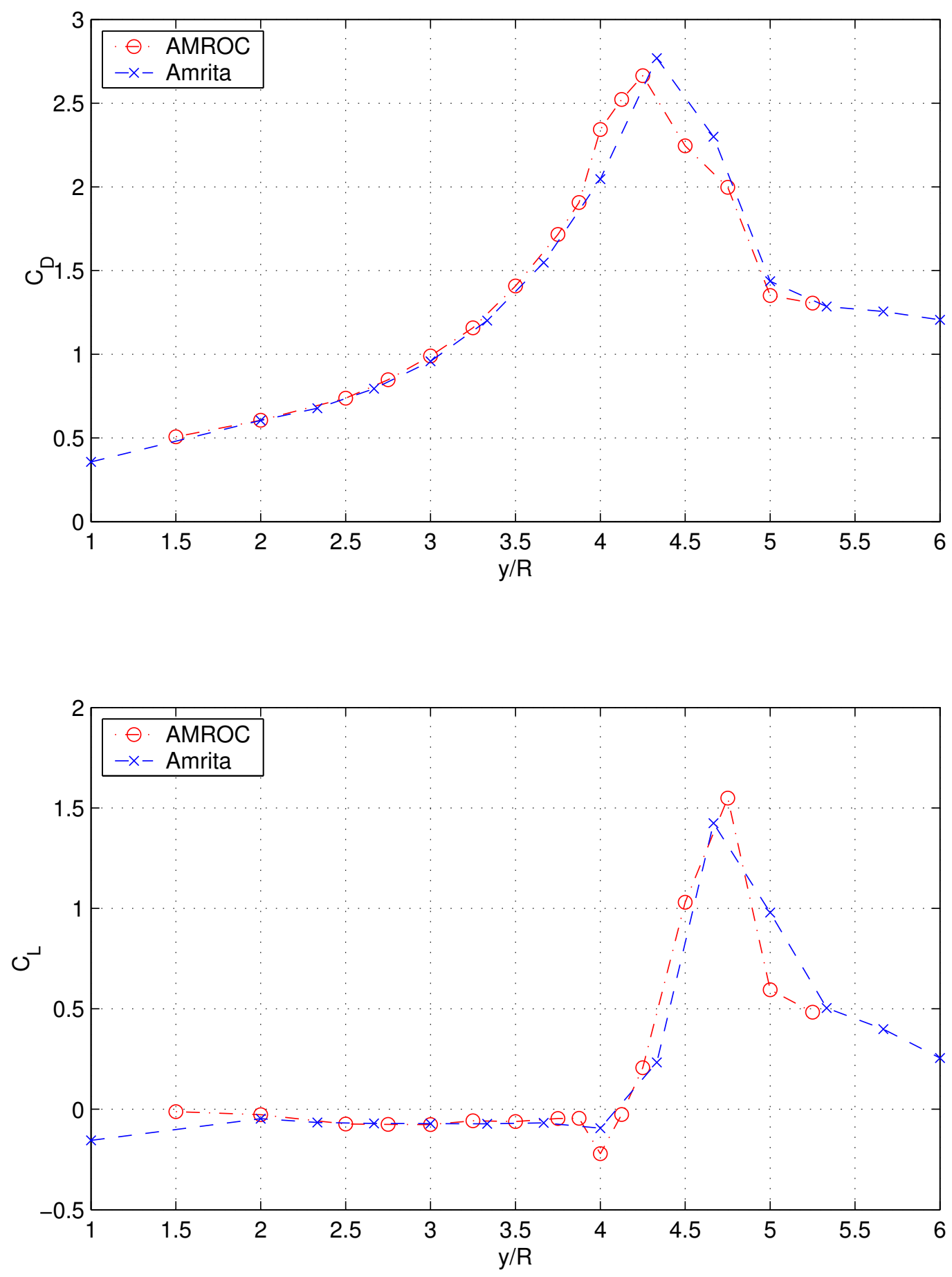

Figure 3.4: Comparison of lift and drag coefficients obtained with Amrita and AMROC 


\subsection{Three-dimensional Computations}

\subsubsection{Introduction}

All three-dimensional simulations were carried out using the AMROC software, but were run at two different facilities. A small number of less-refined computations were run on the CACR machine ASAP, as in the two-dimensional case, while all other computations were run on DataStar at the San Diego Supercomputing Center (SDSC). DataStar is an IBM terascale machine consisting of 176 8-way P655+ nodes, each with 16GB of memory and 7 32-way P690 shared nodes, each with 128GB of memory. Simulations were run on the 8-way nodes - typically between 6 and 16 nodes were used on a given run. The three-dimensional Euler equations were solved for an ideal gas - as in the two-dimensional case, a ratio of specific heats of 1.4 is assumed unless otherwise stated. Initially a hybrid Roe-HLLE scheme with Godunov dimensional splitting was used, but this was found to lead to spurious spatial variations in the freestream flow profile. An exact Riemann solver was subsequently tried, but the best results were obtained with Van-Leer flux vector-splitting, again with Godunov dimensional splitting. This scheme was thus used in all computations presented here.

\subsubsection{Details of Computations}

In all three-dimensional simulations, both bodies were specified as spheres. The parameters that were varied were again the downstream and lateral displacements of the secondary sphere, the ratio of body radii, and the Mach number. For each given combination of downstream displacement, radius ratio, and Mach number, a series of simulations was performed in which the lateral displacement of the secondary body was varied. To maintain consistency with the two-dimensional simulations, we shall refer to the force coefficient in the lateral direction as the lift coefficient, with a 
positive value indicating a repulsive force from the axis of symmetry of the primary body.

Details of the computations are given in Table 3.3. The entries correspond directly to the equivalent two-dimensional entries in Table 3.1, with the exception of the computational overhead entry. The sizes of the computations were such that they could not be completed in a single step without exceeding the Datastar clock limit. They were thus divided into a minimum of two steps, sometimes using differing numbers of CPUs at each step. The computational overhead entry thus includes the total number of CPU hours, with the number in brackets indicating the number(s) of CPUs used for the multiple steps. In particular, as the early steps were used simply to establish the flow, some of the refinement was suppressed, which allowed a smaller number of CPUs to be used. Unfortunately, some of the timing data for the simulations has been lost, hence the empty entry in the fourth column. Also, note that while for the radius ratio 2 simulations, only a single secondary body was included in the computation, for those in which the radius ratio was 4 or 8 , four secondary bodies at different lateral displacements (but sufficiently spaced so as not to interfere with one another) were included in a single computation.

\subsubsection{Results}

Figure 3.5 shows the development of the flow during a typical three-dimensional simulation. The visualisations are computational schlieren images overlaid on the refinement level sets, calculated on the slice plane parallel to the flow that includes the centers of the two bodies. The time instants at which these images are taken are indicated in the plot below, which shows the temporal development of the lift and drag coefficients of the secondary body and the drag coefficient of the primary. The lower right visualisation corresponds to the steady flow solution. As in the two-dimensional case, the steady flow is generated by ramping up the inlet flow speed at constant 


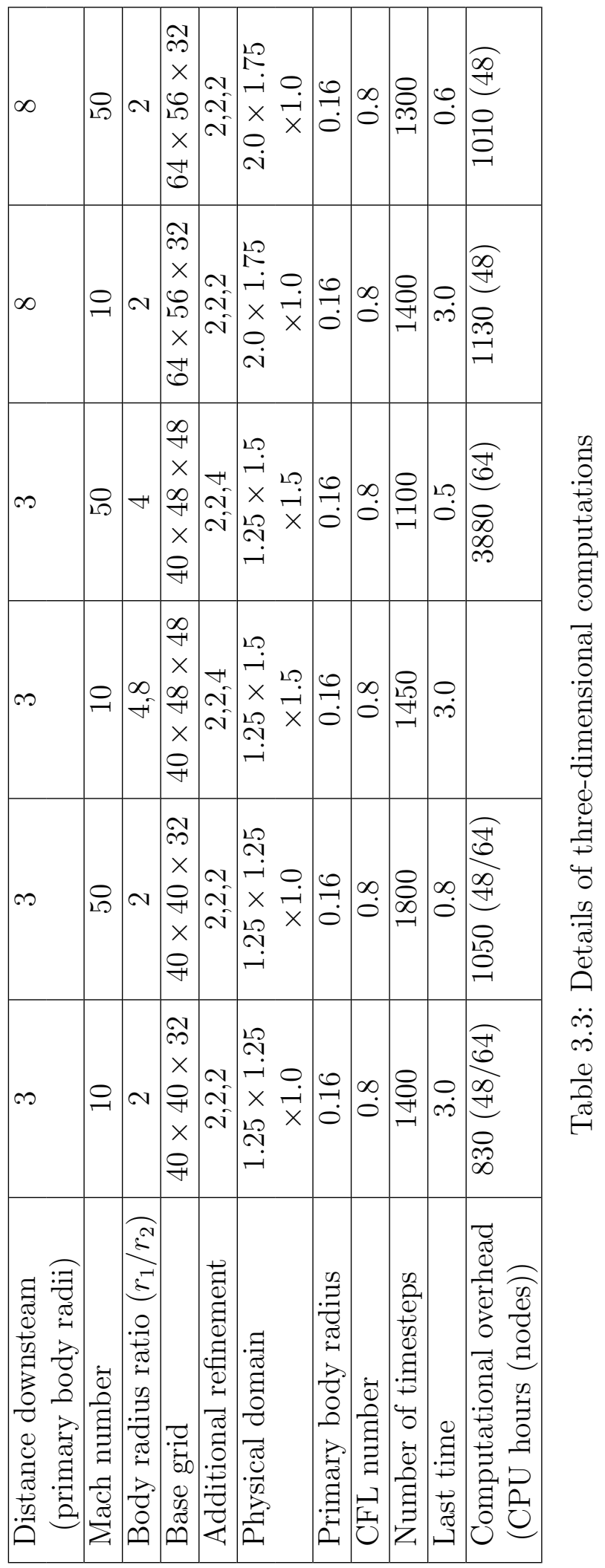


pressure and density until the desired Mach number is obtained. The drag and lift values on the spheres are calculated every 10 timesteps by integrating the pressure on the surface with the appropriate components of the surface normal. Mean values are calculated once the steady flow is established - typically the averaging takes place over the last $10 \%$ of the flow time.

In Figures 3.6 and 3.7 the mean drag and lift coefficients of the secondary body, as functions of lateral displacement, are shown for two combinations of downstream displacement and radius ratio - these are indicated on the schlieren images above. Profiles for Mach numbers of both 10 and 50 are included.

In Figure 3.6 the downstream displacement is 3 primary body radii, and the ratio of body radii is 4 . The drag profile is qualitatively very similar to those seen in the two-dimensional case. The drag coefficient is typically small in the wake region, increases to a maximum value as the lateral displacement is increased, then decreases to the freestream value (of approximately 0.88 ) as the body moves out of the shocked region. The $M=50$ profile climbs slightly more sharply, as the primary shock radius is slightly smaller at higher Mach numbers.

The lift values are typically also small in the wake region and then show a negative tendency as the lateral displacement is increased. As in the two-dimensional case, the lift coefficient jumps sharply once the primary shock impingement point is reached and attains a maximum value when the center of the secondary body is in the vicinity of the primary shock radius. As the body clears the shock, the lift tails off to zero.

The unsteadiness experienced by the secondary body in the wake region is somewhat smaller in the three-dimensional case than the two-dimensional case. In all simulations here the standard deviation of the mean drag value was smaller than $2 \%$. This may be attributed to the generally weaker nature of features such as separation shocks in three dimensions - the extent of the wake region is also smaller than in the two-dimensional case. 

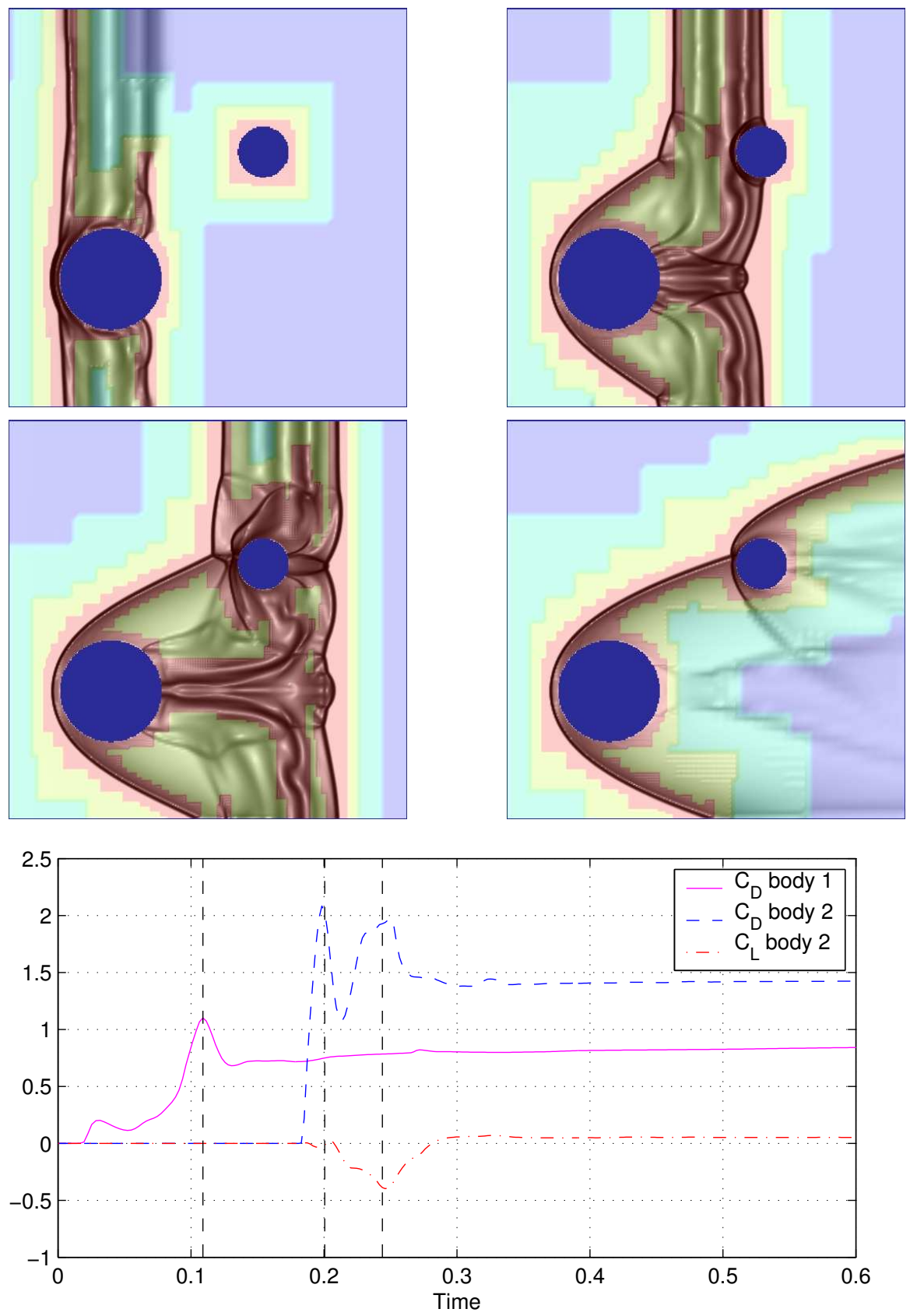

Figure 3.5: Flow development in a typical three-dimensional computation. Computational schlieren images overlaid on refinement level sets are shown at, clockwise from top left, $t=0.109,0.201,3.0,0.244$. The drag and lift coefficient profiles are plotted below with the three earliest times indicated. 

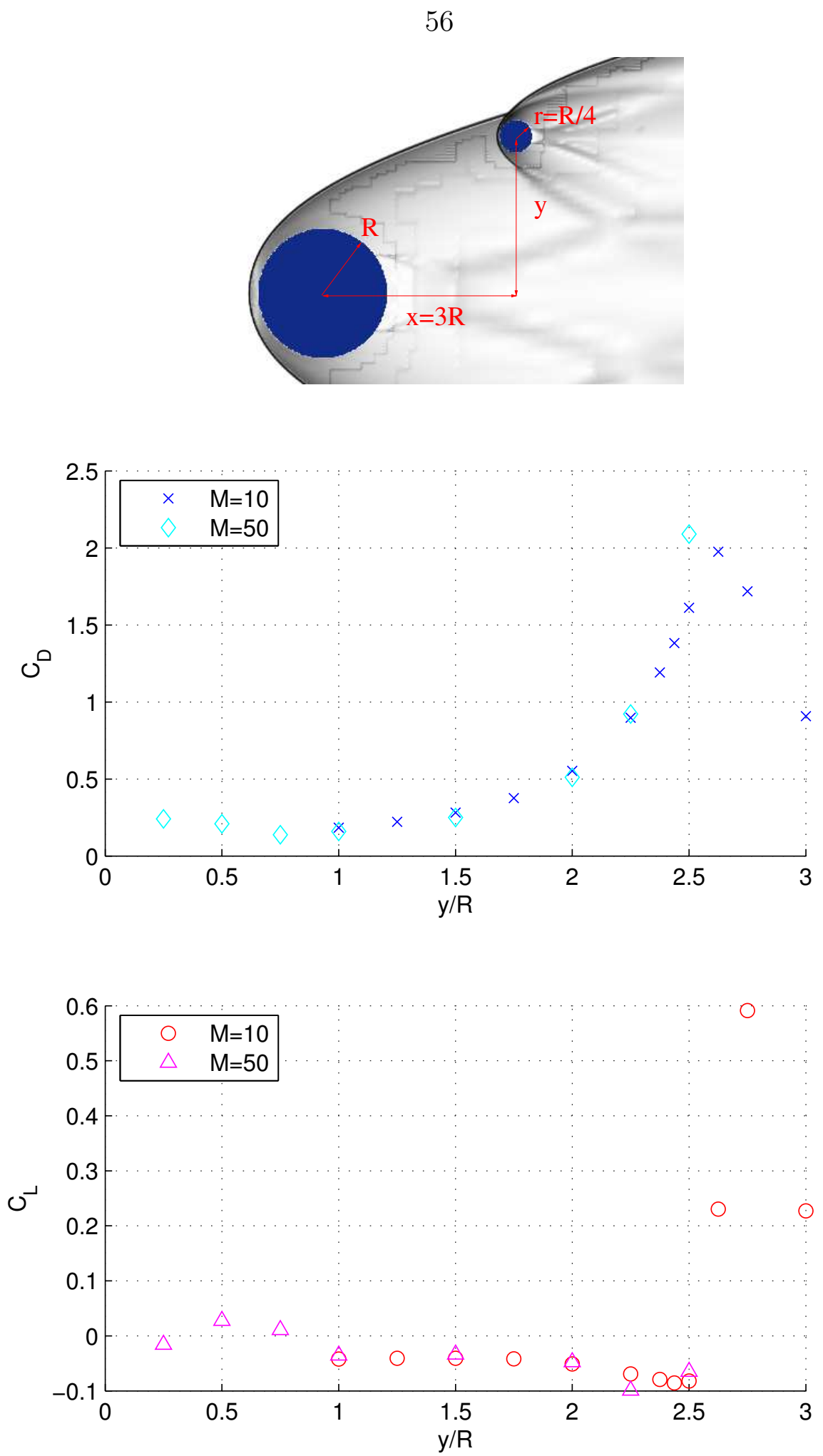

Figure 3.6: Drag and lift coefficients as functions of lateral displacement for spheres in a configuration as shown above. The freestream Mach number in the schlieren image is $M=10$. 
In Figure 3.7 the downstream displacement is 8 primary body radii, and the ratio of body radii 2. The profiles are qualitatively similar to those in Figure 3.6, although we see a greater difference between results for the two Mach numbers. This is another demonstration of the Mach number independence principle becoming more approximate in the far-field.

As no secondary software was available to validate the AMROC computations, instead a set of computations was repeated with one level of refinement discarded. The freestream Mach number for these computations was 10, the ratio of body radii 2, and the downstream displacement of the secondary body 3 primary body radii. The lift and drag coefficients for the two resolutions are compared in Figure 3.8. Agreement between the two is generally good, although there are small discrepancies in the area of primary bow shock impingement. To quantify these discrepancies better, a refinement study was also carried out in three dimensions.

\subsubsection{Refinement Study}

The configuration for the three-dimensional refinement study is shown in the computational schlieren images of Figure 3.5. The downstream and radial displacements (center-to-center) of the secondary body are three and 2.5 primary body radii, respectively, and the freestream Mach number is 10. These values were chosen because, as may be seen in Figure 3.8, in the vicinity of this configuration the lift coefficient is most sensitive to changes in secondary body position (or conversely, changes in shock position). Therefore, this configuration will represent something of a worst-case scenario with regards to the effect of resolution changes on the lift value.

Simulations were carried out for one to four additional levels of refinement over the base grid, and the results are shown in Table 3.4. The $C_{D}$ and $C_{L}$ values are

given in each case, as well as the change in value from that at the previous level of refinement. As may be seen, the values are converging, but the lift especially is quite 

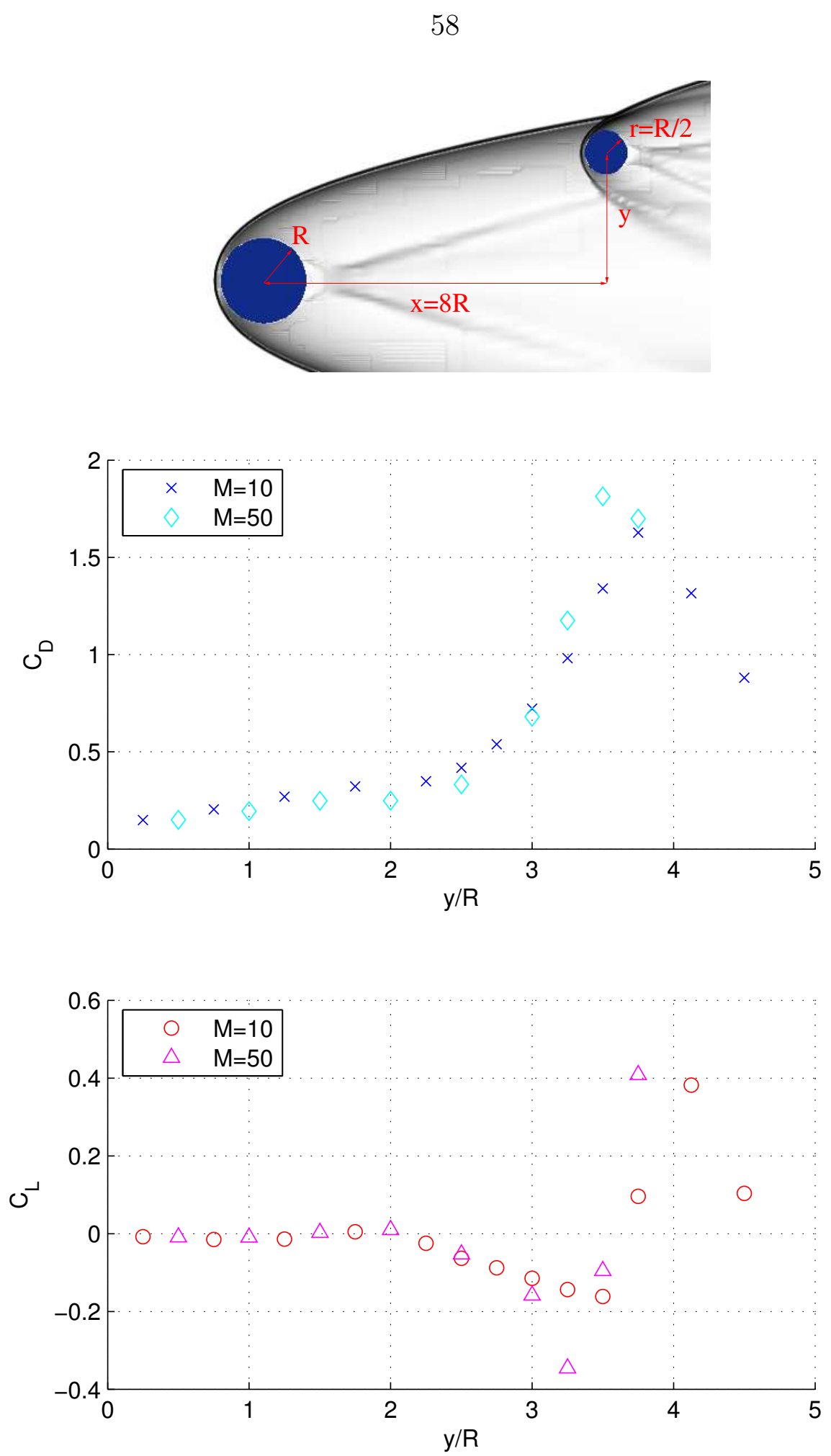

Figure 3.7: Drag and lift coefficients as functions of lateral displacement for spheres in a configuration as shown above. The freestream Mach number in the schlieren image is $M=50$. 


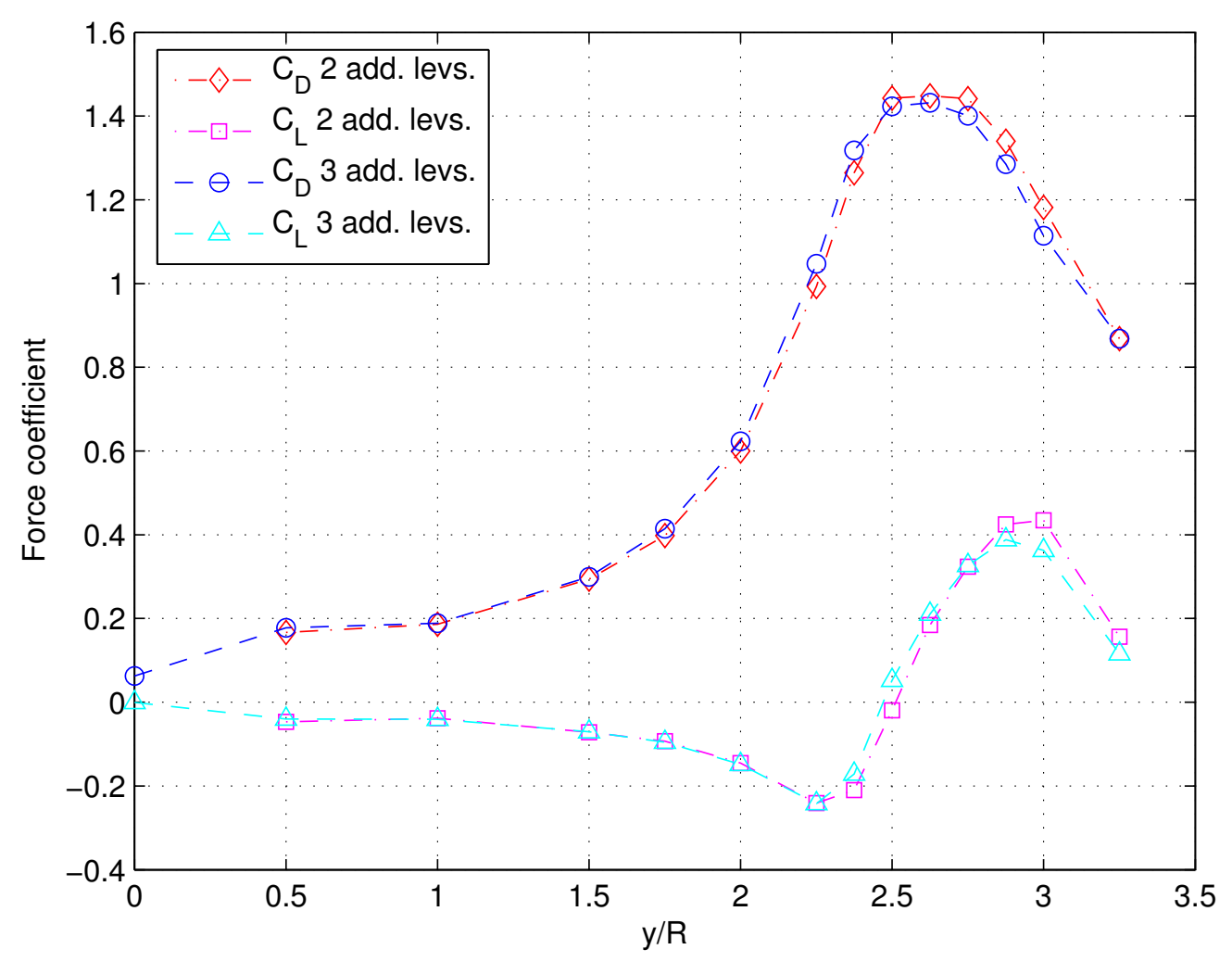

Figure 3.8: A comparison of drag and lift coefficients for 2 and 3 levels of additional refinement. The freestream Mach number is 10, the downstream displacement 3 primary body radii, and the radius ratio 2 .

sensitive to changes in refinement level. In these simulations, the effect of increasing the resolution was to lead to a slight decrease in the primary bow shock radius, or alternatively, a small increase in the effective lateral displacement of the secondary body. This is consistent with the more positive lift values observed at higher levels of refinement.

The computation with four additional levels took approximately $35000 \mathrm{compu}-$ tational hours using $144 \mathrm{CPUs}$ to complete. Thus, given current computing performance, the maximum number of additional levels that can realistically be used is three. If we assume that the lift values continue to converge at the rate indicated by Table 3.4, we obtain a refinement error at this level of refinement that is $5 \%$ of 
the drag value. Similarly, we estimate the refinement error in the drag value to be $2 \%$. These errors will be smaller, however, in configurations that are less sensitive to changes in shock position.

\begin{tabular}{|c|c|c|c|c|}
\hline $\begin{array}{c}\text { Additional. } \\
\text { levels }\end{array}$ & $C_{D}$ & $\Delta C_{D}$ & $C_{L}$ & $\Delta C_{L}$ \\
\hline 1 & 1.264 & & -0.176 & \\
2 & 1.442 & 0.178 & -0.019 & 0.157 \\
3 & 1.423 & -0.019 & 0.052 & 0.071 \\
4 & 1.408 & -0.015 & 0.087 & 0.035 \\
\hline
\end{tabular}

Table 3.4: $C_{D}$ and $C_{L}$ values for the three-dimensional refinement study. $\Delta$ indicates the difference between the value at the current and the previous level of refinement. 


\section{Chapter 4}

\section{Comparison of Theoretical and Computational Results}

We are now in a position to see how well the analytical methodology developed in Chapter 2 performs. In this chapter we will compare the analytical drag and lift coefficients with those obtained in the numerical simulations of Chapter 3.

\subsection{Two-Dimensional Comparison}

To begin with, we will compare coefficients with the lateral displacement of the secondary body non-dimensionalized by the primary body diameter. In Figure 4.1, the theoretical coefficients obtained using the Gaussian profile with no $x$-derivatives are plotted along with computational values for Mach numbers of 10 and 50. The body diameter ratio, $d_{1} / d_{2}$, is 2 , and the downstream displacement (center-to-center) is $2 d_{1}$. This corresponds to a $x / d_{1}$ value of approximately 2.75 , as the shock stand-off distance is approximately half a body radius.

As may be seen, agreement between the theoretical and computational values is poor. The reason for this may be seen from the dashed vertical lines, which indicate the shock radius in each case at this distance downstream (for the computational cases, the shock radius was estimated from visualizations of the computed flow; the theoretical value is given by Equation 2.5). The blast wave analogy significantly 
underestimates the shock radius, and, as the shock provides the boundary for qualitatively different flow regions, this results in a large discrepancy in the lift and drag values. This suggests that the more appropriate non-dimensionalization for the lateral displacement is the shock radius in each case. The profiles resulting from this non-dimensionalization are shown in Figures 4.2 through 4.5 for combinations of body diameter ratios of 2 and 6 with downstream displacements (center-to-center) of 2 and $4 d_{1}$. The latter downstream displacement value corresponds to an $x / d_{1}$ value of approximately 4.75. In all cases, computational profiles at Mach numbers of both 10 and 50 are included.

First, we should make note of the regions in which we do not expect the blast wave analogy model to perform well. For $r / R_{s}$ close to 1 , the primary bow shock will impinge on the secondary body and significantly affect the pressure distribution. In each of the computational profiles, we see that this impingement causes a large increase in the lift coefficient, for reasons previously outlined. The effect of the impingement on the drag coefficient is less significant, as the main contribution to the drag comes from the region near the stagnation point, which does not feel the impingement effects until larger values of $r / R_{s}$ are reached.

For small values of $r / R_{s}$, on the other hand, the secondary body is in the wake of the primary body, or for slightly larger values, interacts with the primary separation shock. This interaction was noted in Chapter 3 and may be seen in the hump in the drag profile in each case at around $r / R_{s} \approx 0.2-0.4$. As neither the wake region nor the separation shock is present in the blast wave analogy, it is not surprising that there are discrepancies between the theoretical model and computations for small $r / R_{s}$

For $r / R_{s}$ not too close to either 0 or 1 , however, we see that the blast wave model does a reasonably good job of predicting the drag and lift coefficients. Qualitatively, the trends are very well captured - note that the effect of body size predicted by the 

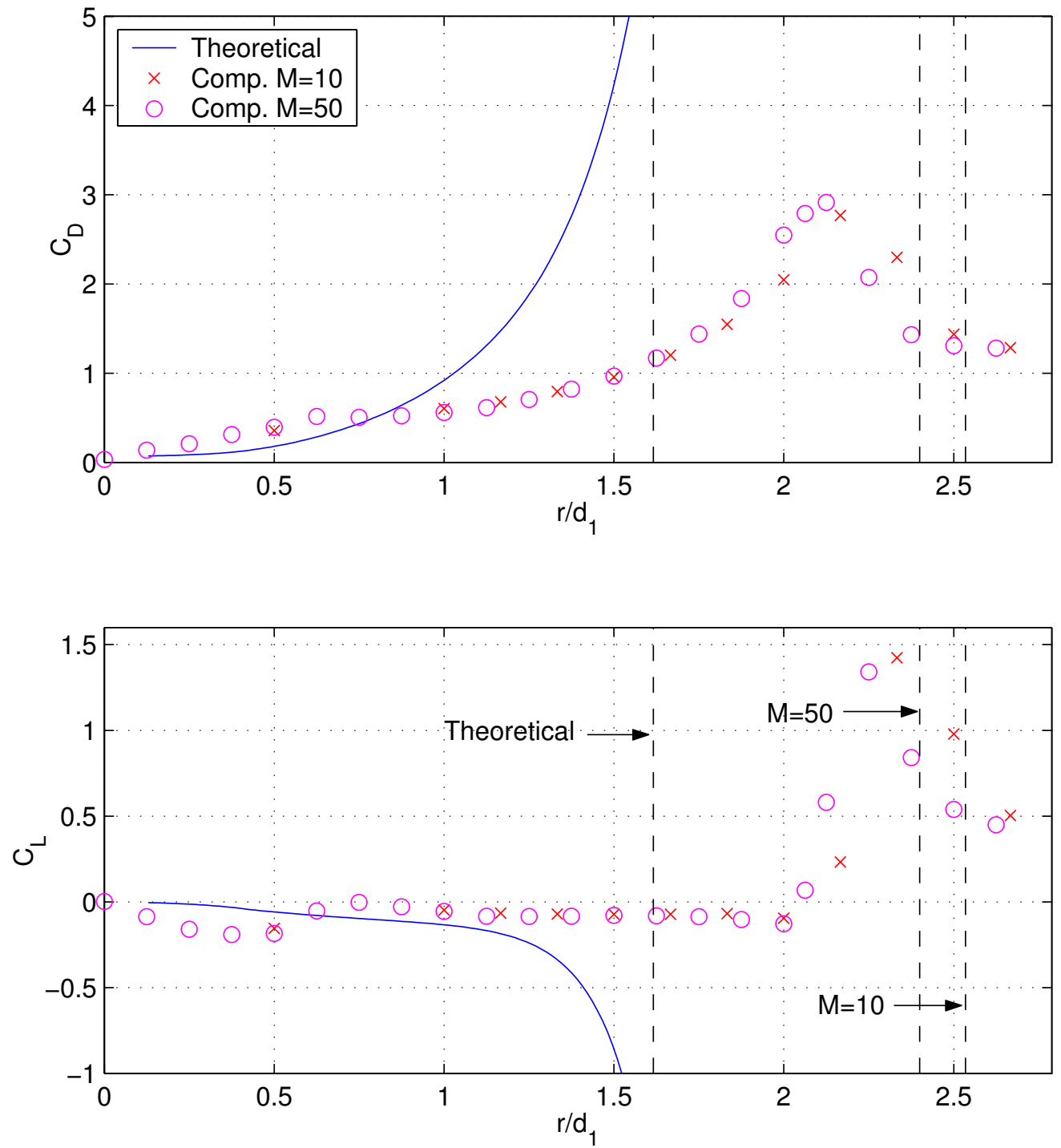

Figure 4.1: Theoretical and computed drag and lift coefficients in two dimensions for $M=10$ and $50, d_{1} / d_{2}=2$, at a downstream displacement of $2 d_{1}$ (center-to-center). The lateral displacement has been normalized by the primary body diameter, and the dashed vertical lines indicate the position of the primary bow shock in each case. 
theoretical model is very much present in the computations. The model also predicts such details as the lift coefficient crossing from positive to negative as the lateral displacement is decreased for the two $d_{1} / d_{2}=6$ cases (Figures 4.3 and 4.5). In Figure 4.4 we also notice an interesting effect. It was in this configuration in the previous chapter that we noted slight qualitative differences in the two computational lift profiles. The theoretical profiles mirror these differences, with the modified Newtonian profile lying closer to the $M=10$ values and the Gaussian profile better approximating the $M=50$ values. This is consistent with our earlier prediction that the Gaussian profile should be more appropriate at higher Mach numbers.

Overall, the modified Newtonian description without $x$-derivatives seems to do the best job of modeling the lift and drag coefficients. The inclusion of the $x$-derivatives for either distribution results in predicted drag coefficients that are too high. The Gaussian description (without $x$-derivatives) does well in most cases, but notably in Figure 4.2 the lift coefficient profile falls too quickly with increasing $r / R_{s}$. In the other cases, however, both the modified Newtonian and Gaussian descriptions allow reasonable quantitative predictions to be made.

\subsection{Three-Dimensional Comparison}

We begin again by comparing theoretical and computational profiles in which the secondary lateral displacement has been non-dimensionalized by the primary body diameter. In Figure 4.6 the theoretical coefficients calculated with the Gaussian profile (with no $x$-derivatives) are compared with those generated by AMROC computations for a ratio of body diameters of 2 and a downstream displacement (center-to-center) of 1.5 primary body diameters. This downstream displacement corresponds to a $x / d_{1}$ value of approximately 2.07 - the shock stand-off distance is measured from visualisations of the computed flow. Computational profiles with freestream Mach numbers 

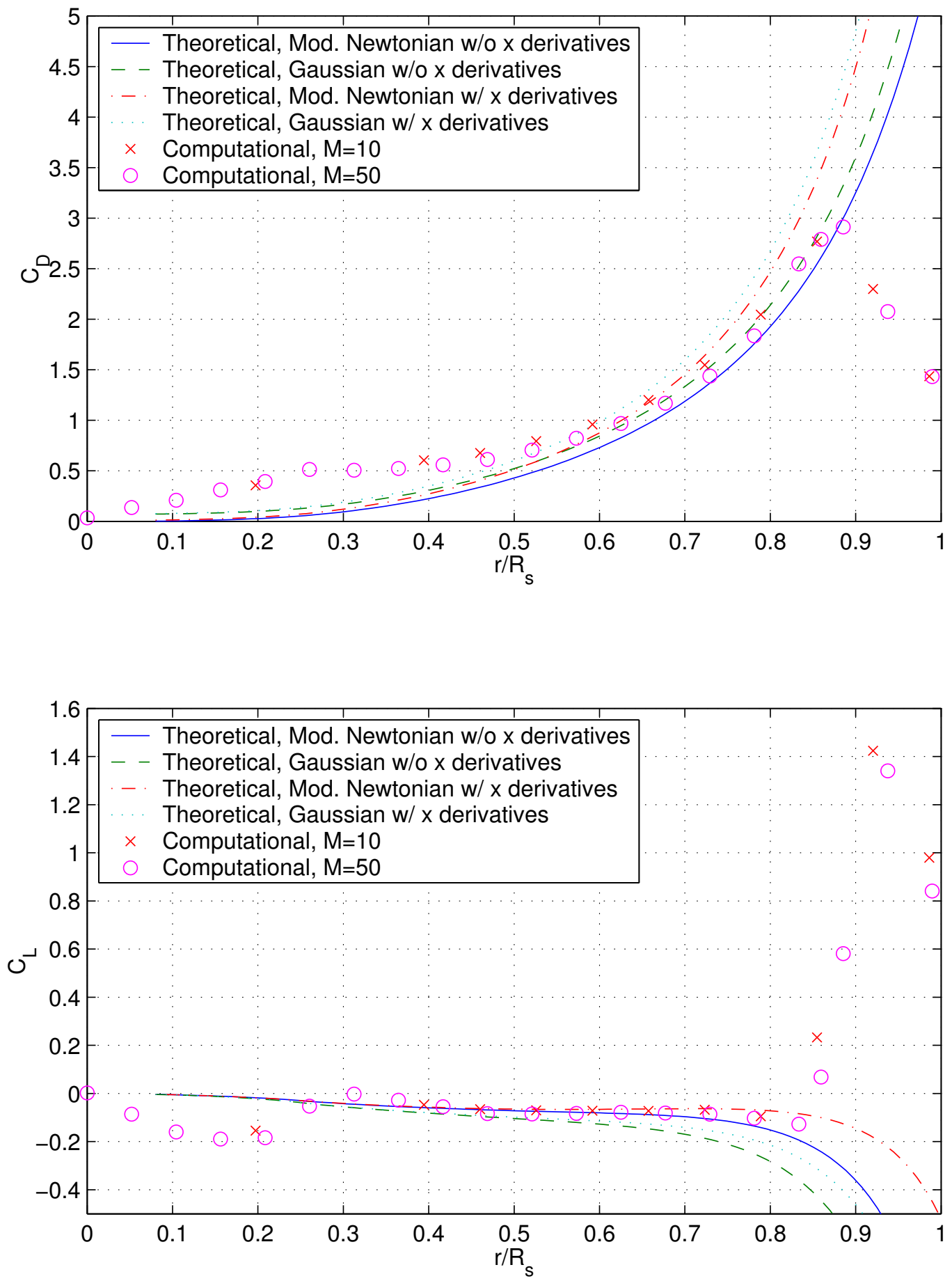

Figure 4.2: Theoretical and computed drag and lift coefficients in two dimensions with the lateral displacement normalized by respective shock radii for $M=10$ and $50, d_{1} / d_{2}=2$, and a downstream displacement of $2 d_{1}$ (center-to-center). 

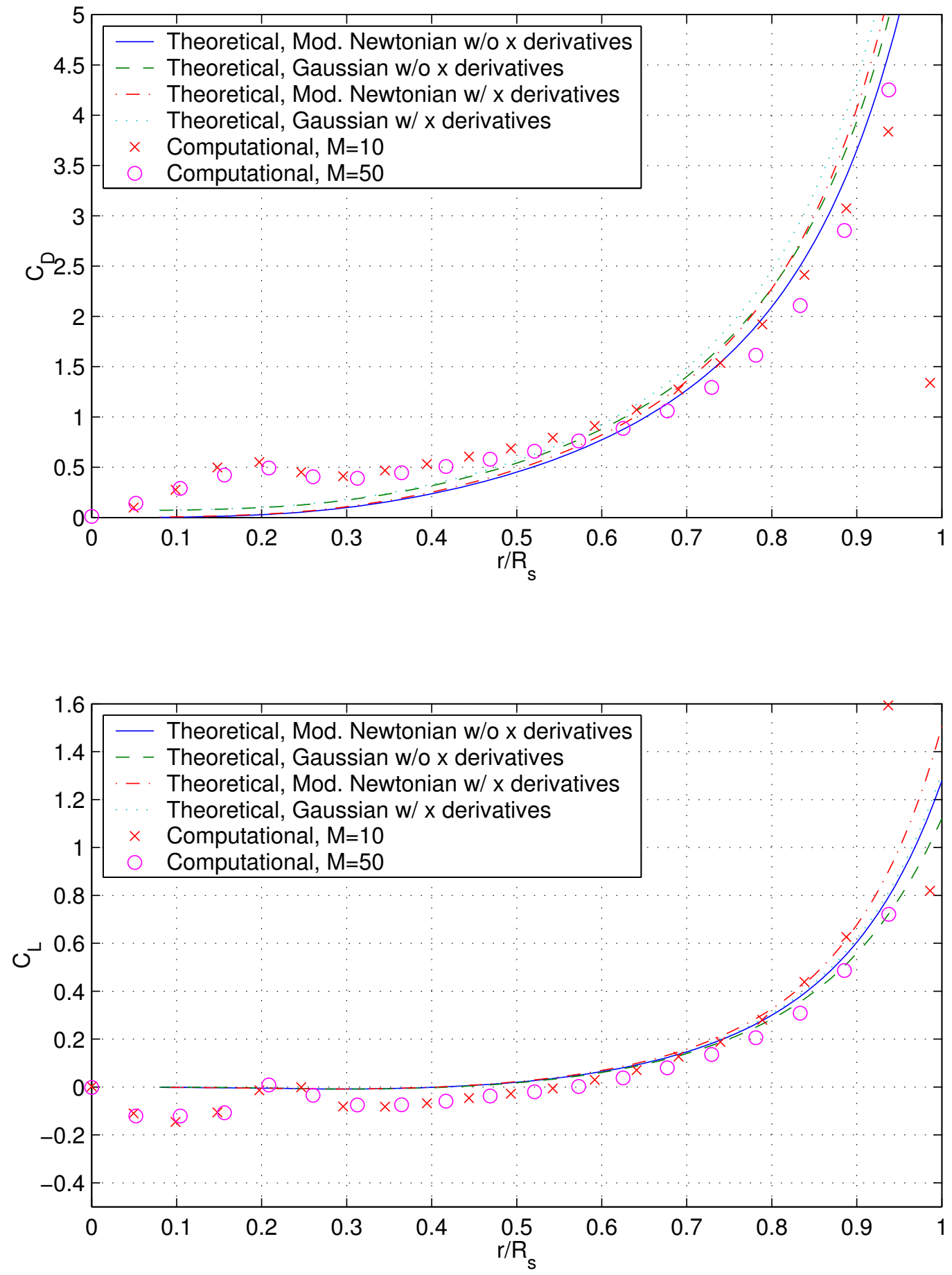

Figure 4.3: Theoretical and computed drag and lift coefficients in two dimensions for $M=10$ and $50, d_{1} / d_{2}=6$, and a downstream displacement of $2 d_{1}$. 

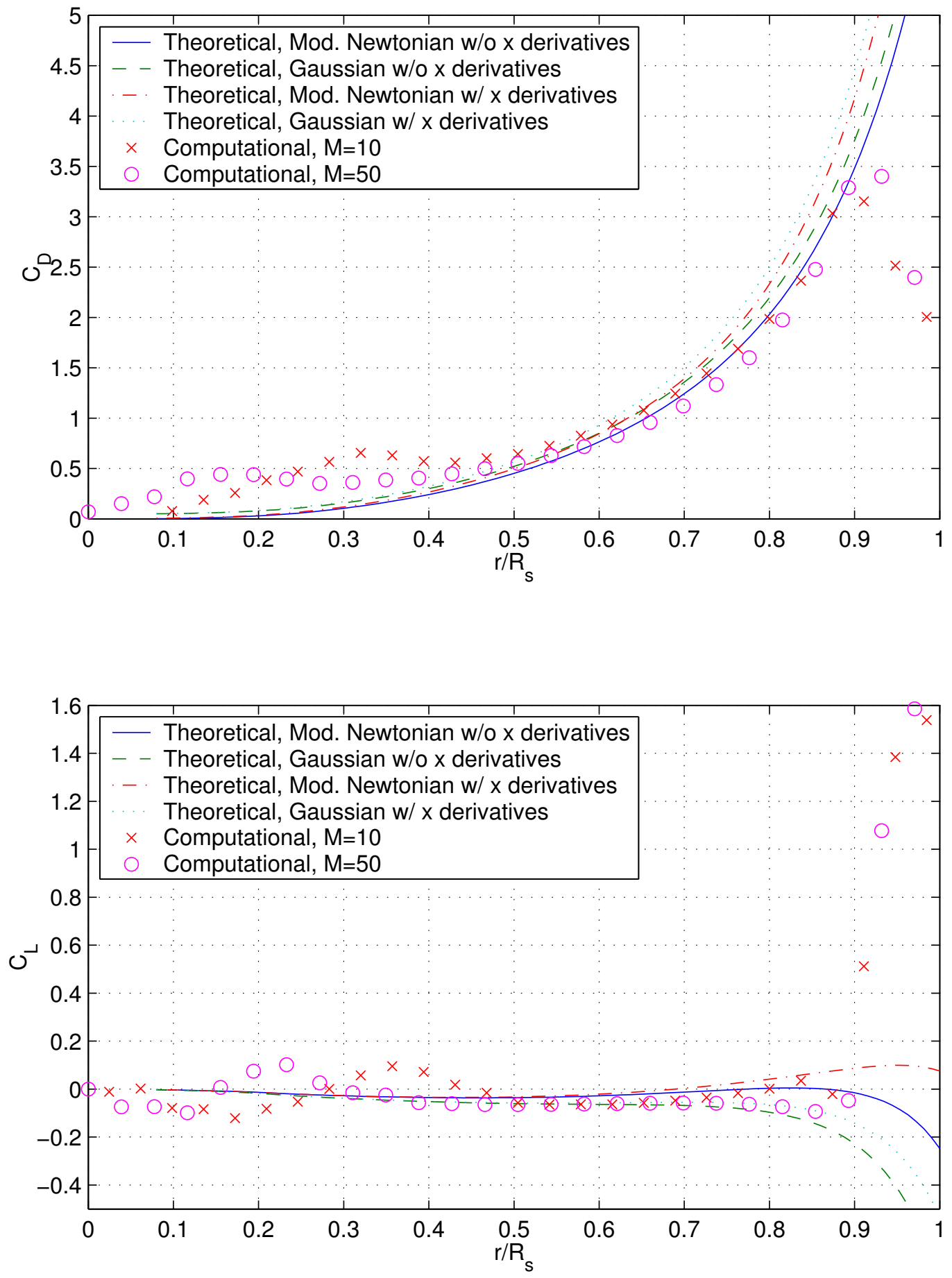

Figure 4.4: Theoretical and computed drag and lift coefficients in two dimensions for $M=10$ and $50, d_{1} / d_{2}=2$, and a downstream displacement of $4 d_{1}$. 

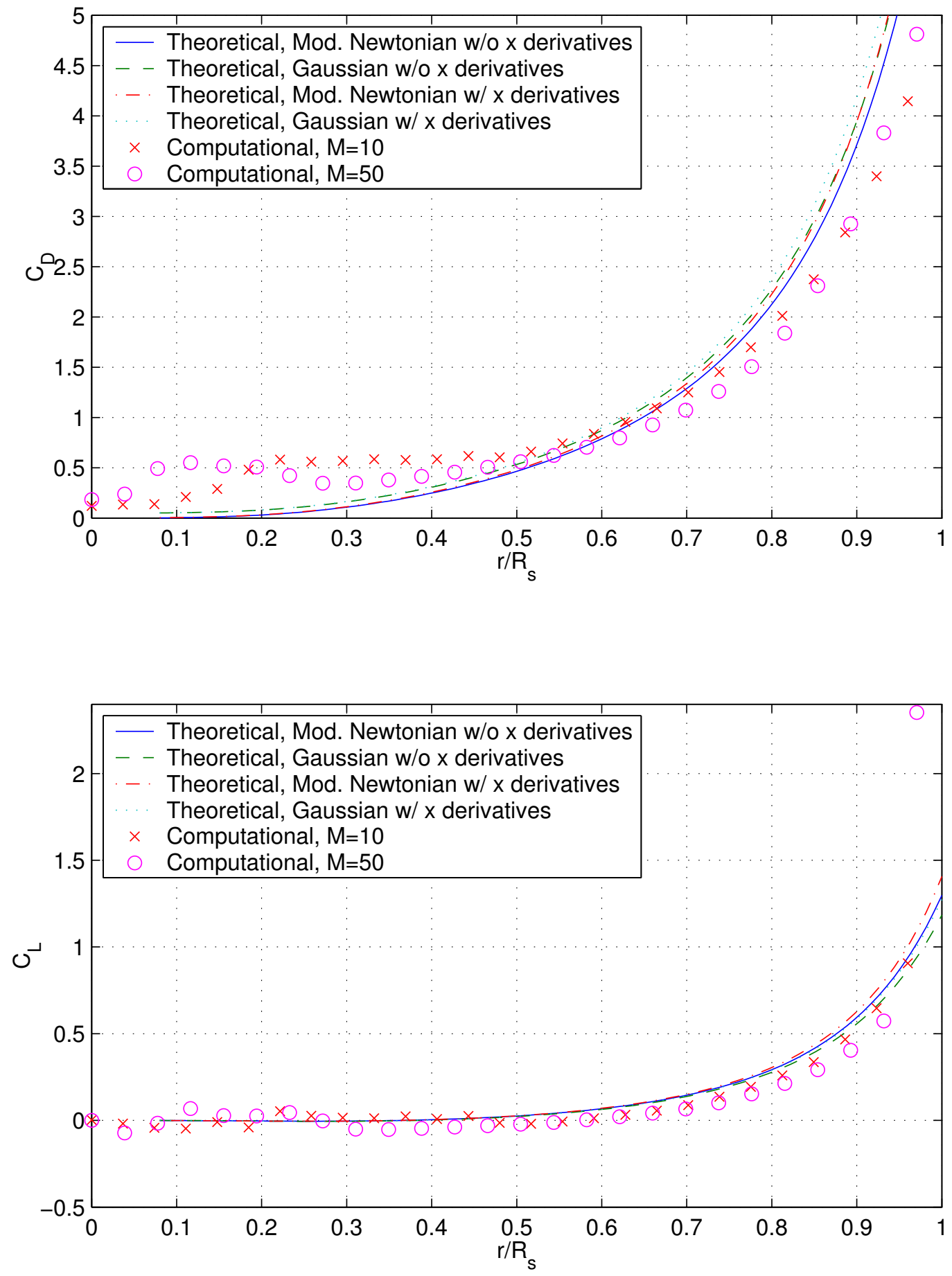

Figure 4.5: Theoretical and computed drag and lift coefficients in two dimensions for $M=10$ and $50, d_{1} / d_{2}=6$, and a downstream displacement of $4 d_{1}$ 
of 10 and 50 are included. As in the two-dimensional case, non-dimensionalization by the primary body size does not give good agreement between the theoretical and computational values. The problem is once again a disagreement in primary shock radii, as indicated by the dashed vertical lines.

We thus try normalizing again by the shock radius in each case: the results are shown in Figures 4.7 through 4.10. Once again, agreement is much improved with this normalization. For $r / R_{s}$ close to 0 and 1 , the expected discrepancies appear as a result of the secondary's interaction with the wake region and the primary bowshock, but away from these extremes agreement is quite reasonable. Agreement with the $M=50$ computational values is better than that for the $M=10$ values, as might be expected. As in the planar case, the Newtonian profiles appear in general to capture the computational values better than the Gaussian profiles do. In Figure 4.7 the profiles that include $x$-derivatives show better agreement than those without, but in other cases this is not as obvious. Note, however, that the further downstream the secondary body is, the smaller the effect of including the $x$-derivatives will be, as all derivatives will decay as the shock radius grows. The inclusion of the $x$-derivatives will also become less important as the secondary body size is decreased.

The most significant problem with the theoretical profiles under the current normalization seems to be that the predicted drag profile (and in some cases the lift also) decays too rapidly as $r / R_{s}$ is decreased from 1 , leading to smaller values than those predicted by the computations. This is most likely caused by the $p_{\text {stag }}^{\prime}$ profile predicted by the blast wave analogy decaying more rapidly than that in the computed flow. 

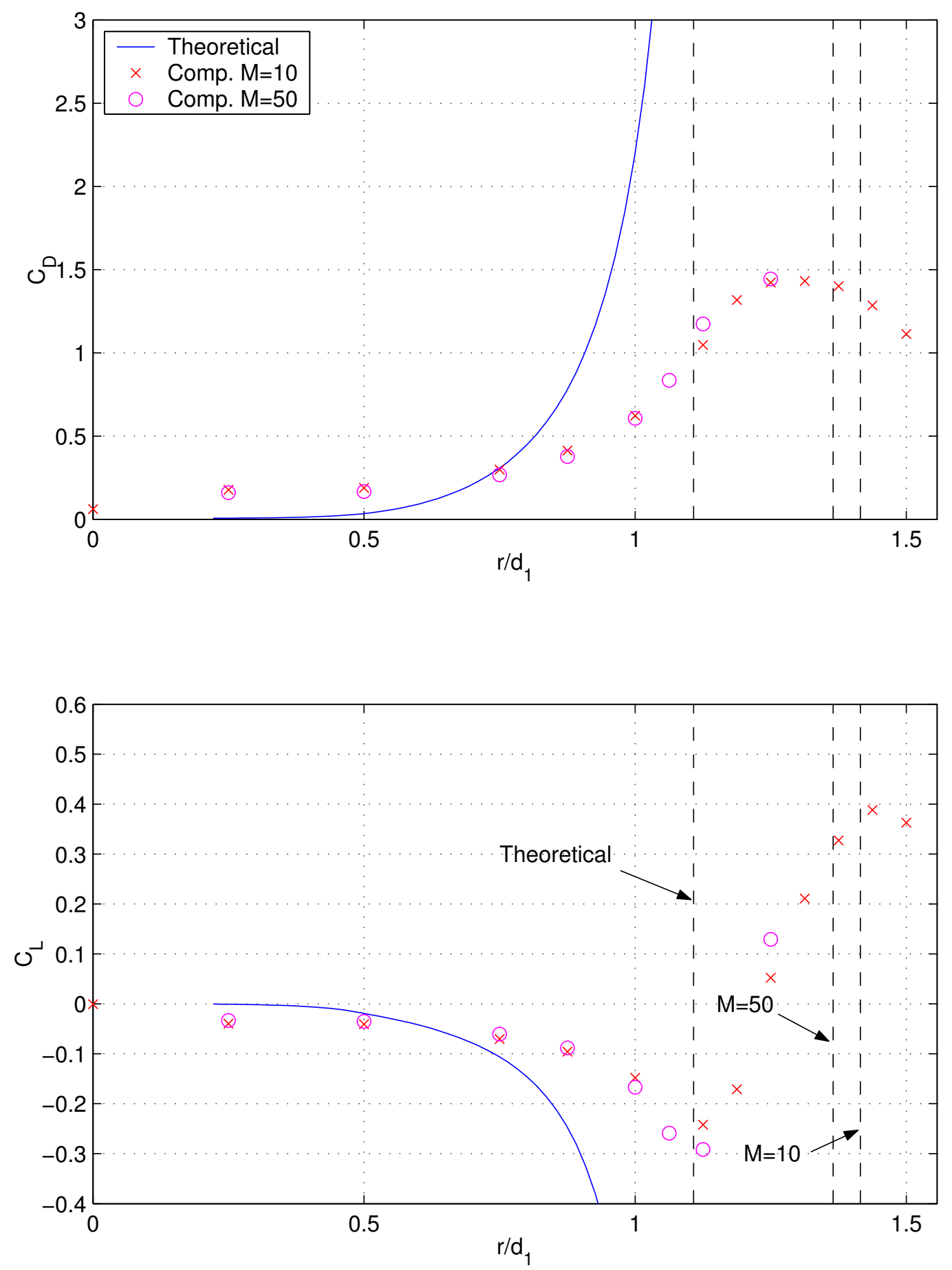

Figure 4.6: Theoretical and computed drag and lift coefficients in three dimensions for $M=10$ and $50, d_{1} / d_{2}=2$ at a downstream displacement of $1.5 d_{1}$ (center-to-center). The lateral displacement has been normalized by the primary body diameter, and the dashed vertical lines indicate the position of the primary bow shock in each case. 

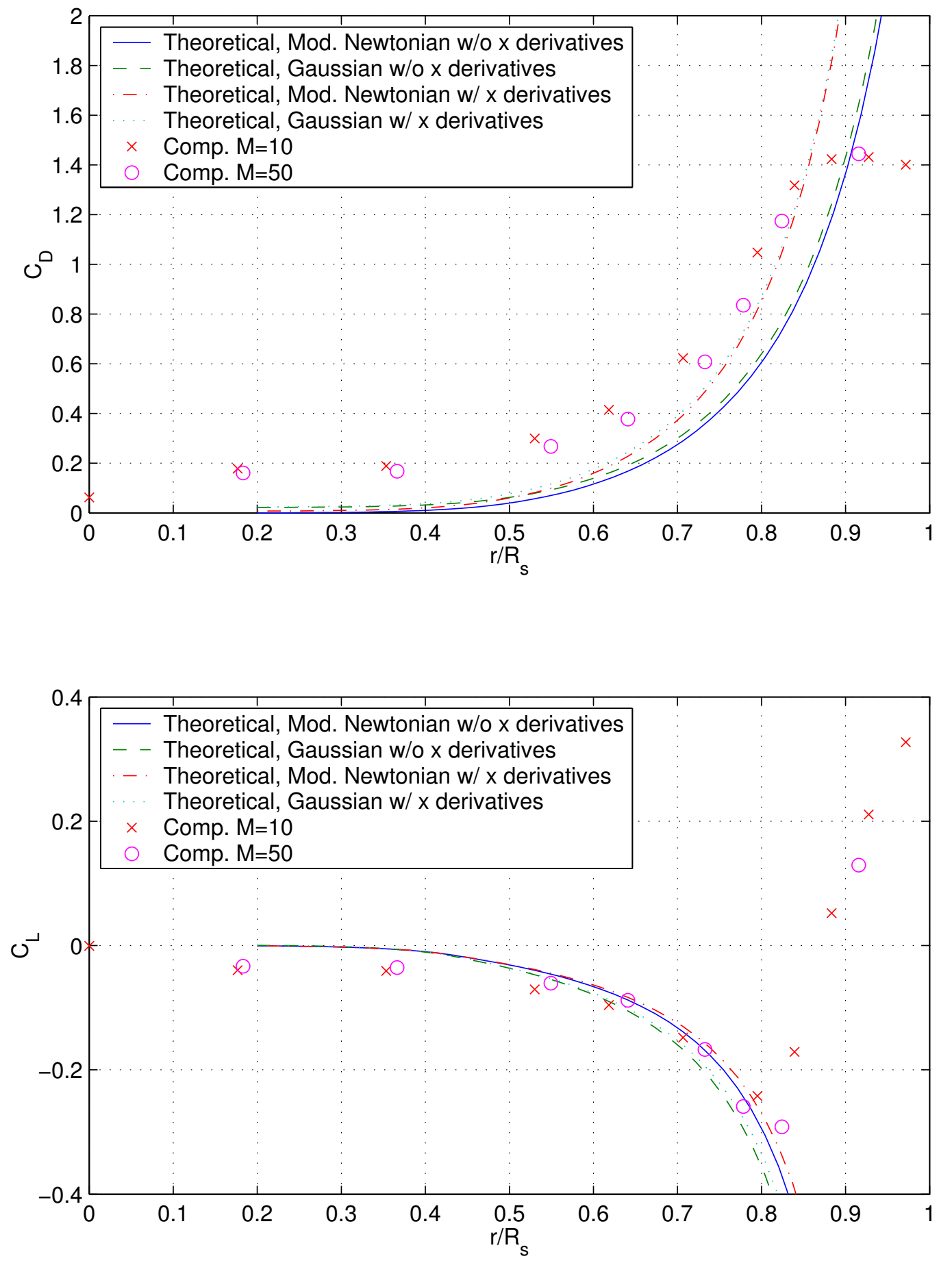

Figure 4.7: Theoretical and computed drag and lift coefficients in three dimensions with the lateral displacement normalized by the respective shock radii for $M=10$ and $50, d_{1} / d_{2}=2$, and a downstream displacement of $1.5 d_{1}$ (center-to center). 

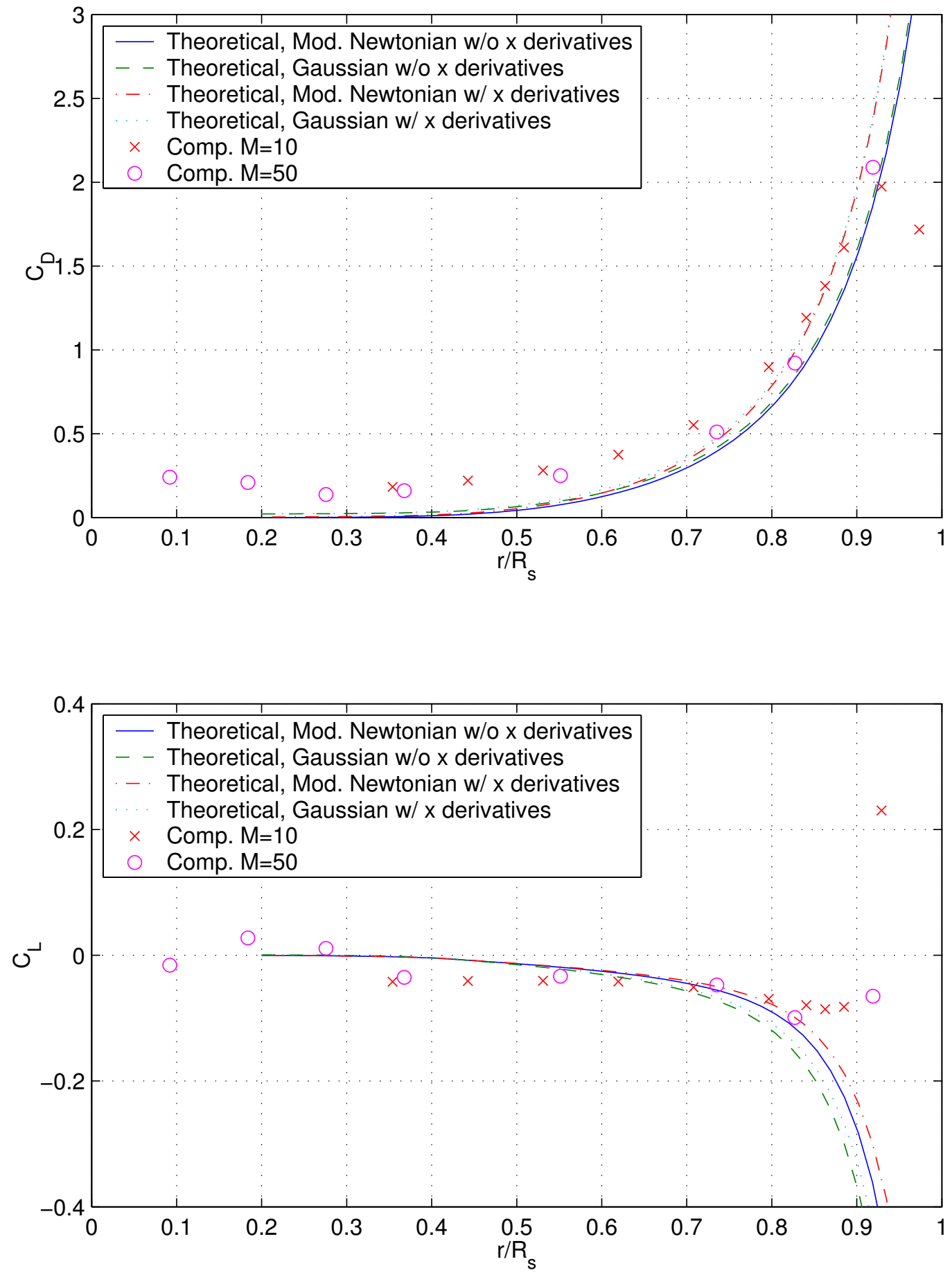

Figure 4.8: Theoretical and computed drag and lift coefficients in three dimensions for $M=10$ and $50, d_{1} / d_{2}=4$, and a downstream displacement of $1.5 d_{1}$. 

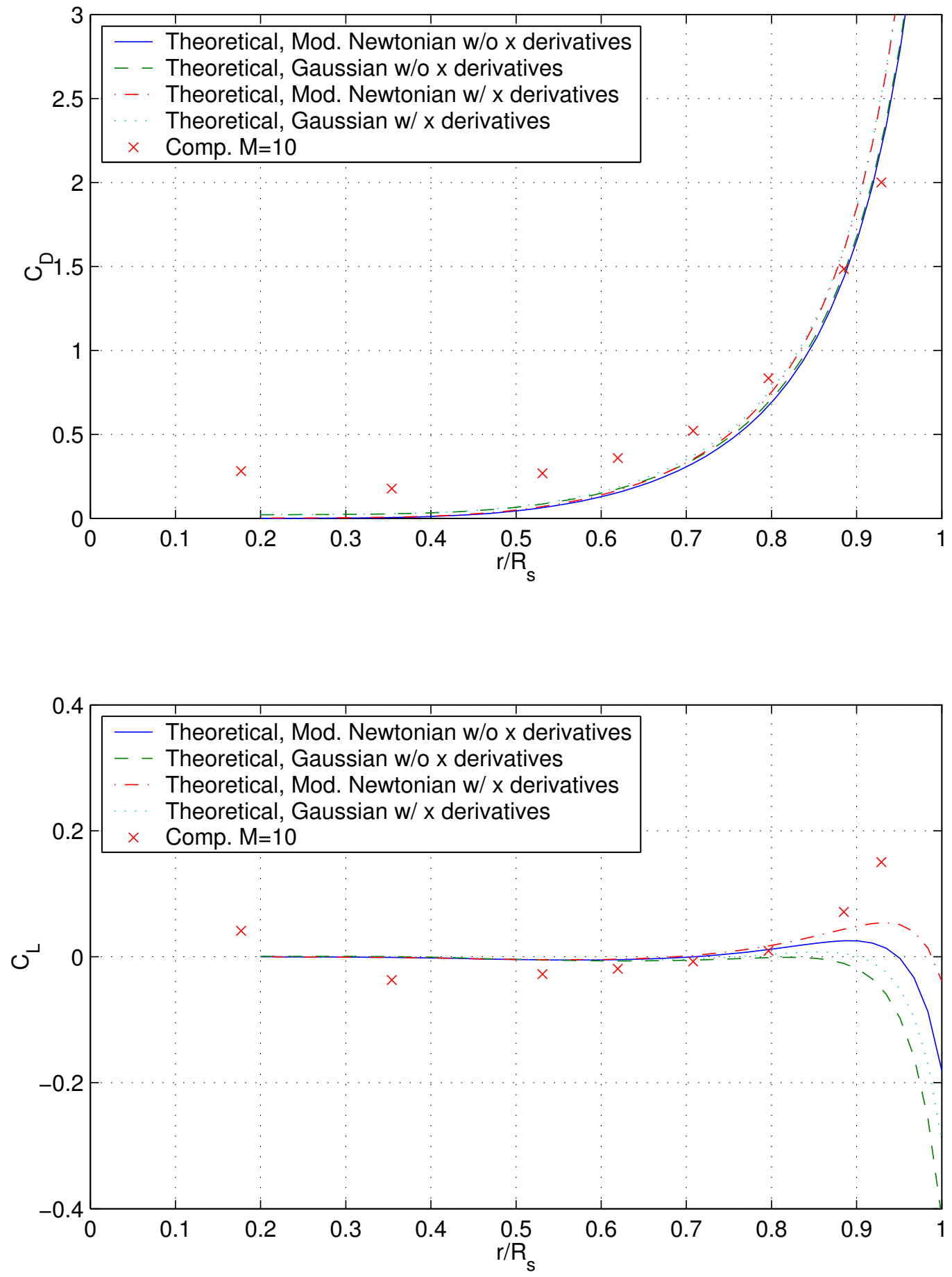

Figure 4.9: Theoretical and computed drag and lift coefficients in three dimensions for $M=10$ and $50, d_{1} / d_{2}=8$, and a downstream displacement of $1.5 d_{1}$. 

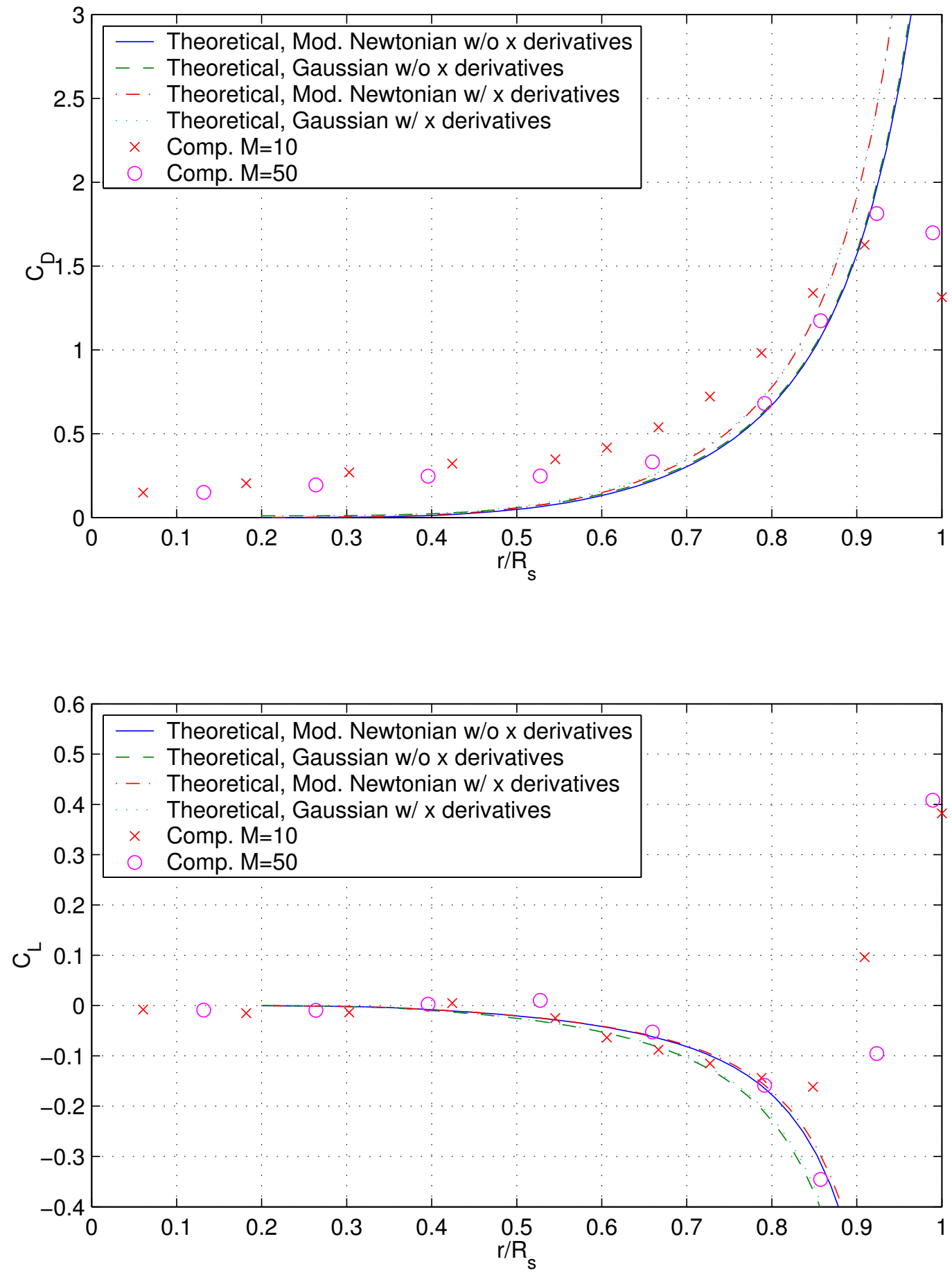

Figure 4.10: Theoretical and computed drag and lift coefficients in three dimensions for $M=10$ and $50, d_{1} / d_{2}=2$, and a downstream displacement of $4 d_{1}$. 


\subsection{Discussion}

As we have seen, the theoretical profiles in both two and three dimensions provide reasonable approximations to the computational values, but only once the lateral displacements have been normalized by the appropriate shock radii. This does limit the predictive power of the analytical methodology somewhat. If a given physical situation is to be modeled by the methodology, the physical value of $R_{s}$ must be determined independently. This would be possible through an experimental or numerical simulation, but then one may ask why not simply simulate the physical configuration directly, rather than carry out a simulation to determine the shock radius. Of course, a single simulation in the latter case would yield a shock shape, which would then enable the methodology to be employed over a range of secondary body positions, whereas the former simulation would yield only the forces at a single position.

As an alternative to a full simulation, numerical or experimental, several authors have proposed empirical correlations to the shock shape produced by a supersonic blunt body based on experimental data. The detached shock wave is assumed to take the form of a hyperbola that is asymptotic to the freestream Mach angle. The equation for the coordinates of the shock is

$$
x=R+A-R_{c} \cot ^{2} \theta\left[\left(1+\frac{y^{2} \tan ^{2} \theta}{R_{c}^{2}}\right)^{1 / 2}-1\right],
$$

where $\theta$ is the shock angle, $A$ is the shock stand-off distance, and $R_{c}$ is the shock radius of curvature at the vertex. Ambrosio and Wortman (1962) gave correlations for the shock stand-off distance based on experimental data from several sources:

$$
\begin{aligned}
\text { Spheres : } A / R & =0.143 \exp \left(3.24 / M^{2}\right) \\
\text { Cylinders : } A / R & =0.386 \exp \left(4.67 / M^{2}\right) \text {. }
\end{aligned}
$$


Similarly, Billig (1967) obtained correlations for the vertex radius of curvature:

$$
\begin{gathered}
\text { Spheres : } \left.R_{c} / R=1.143 \exp \left[0.54 /(M-1)^{1.2}\right)\right] \\
\text { Cylinders : } R_{c} / R=1.386 \exp \left[1.8 /(M-1)^{0.75}\right] .
\end{gathered}
$$

These relations could be used to provide a more accurate shock radius to which the blast wave methodology could be applied.

As a final alternative, we have seen that the problem under current discussion arises from the underprediction of the shock radius by the blast wave analogy. This problem is well-documented, however, and is understood to arise from the fact that in the blast wave analogy the energy in the flow originates from a point source, whereas any physical body has a finite extent. In effect, the shock in the physical situation is displaced outwards from the point explosion solution by the body, resulting in a larger shock radius. It thus seems reasonable to propose an effective origin for the shock radius in the blast wave solution. This origin would be displaced outwards from the existing origin and could be useful in obtaining a more accurate value for the shock radius in the intermediate- and far-fields. This idea will be explored further in Section 6.2. 


\section{Chapter 5}

\section{Experimental Investigation}

\subsection{Introduction}

In order to further investigate the proximal bodies problem, and to provide verification for the numerical simulations, a series of experiments has been performed in the T5 hypervelocity shock tunnel facility at Caltech. The main objective of the experimental investigation was to measure the forces on a secondary sphere in configurations similar to those simulated in Chapter 3. As outlined in Chapter 1, there is a dearth of reliable force- and moment-measurement techniques available for short duration hypersonic facilities: thus, as part of the experimental investigation, the development of new techniques was required.

\subsection{Experimental Procedure}

\subsubsection{The T5 Hypervelocity Shock Tunnel Facility}

The T5 hypervelocity shock tunnel is one in a series of free-piston driven facilities of the type pioneered by Stalker (1961). A schematic of the facility with enlargements of the important components is shown in Figure 5.1.

The operational principle of T5 and other similar facilities is as follows. Compressed air, stored in the secondary reservoir $(2 \mathrm{R})$, is used to accelerate a free piston 
78

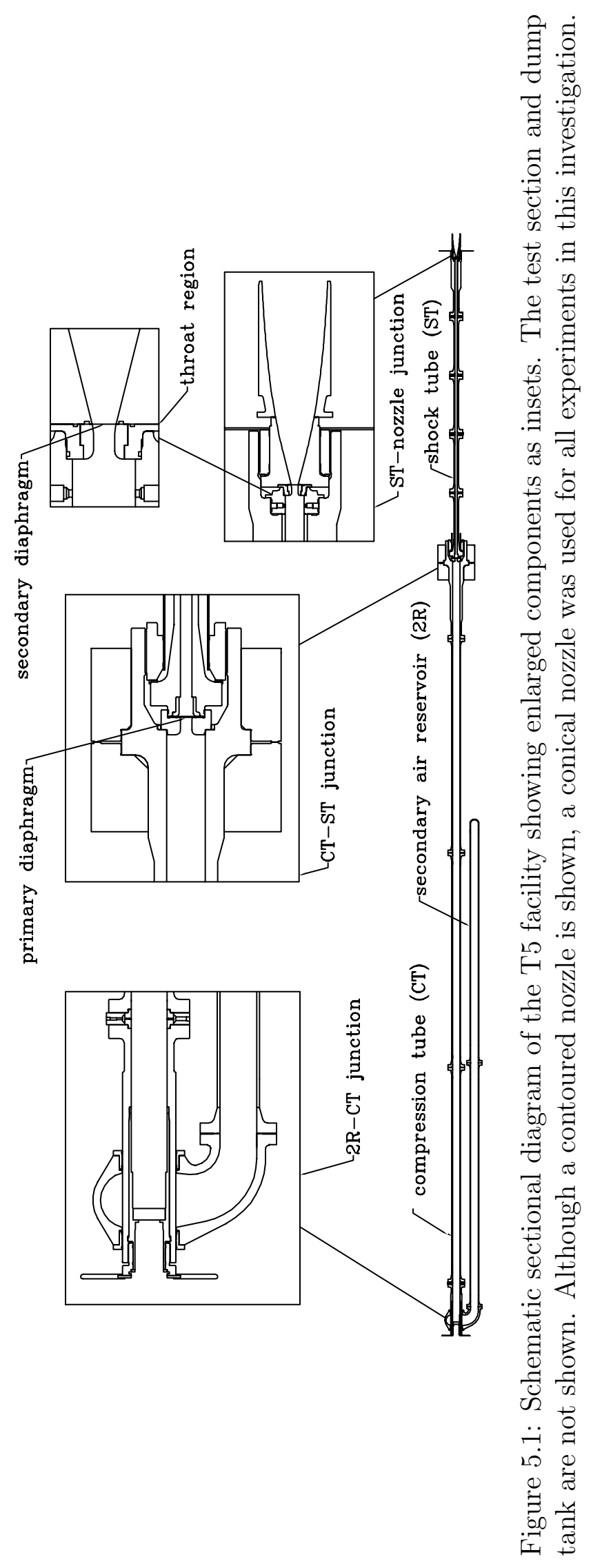


down the compression tube (CT), adiabatically compressing the CT gas ahead of it. This gas, typically a mixture of helium and argon, is then used as the driver gas for the shock tube $(\mathrm{ST})$. The $\mathrm{ST}$ is filled with the test gas at low pressure and room temperature and is separated from the CT by a stainless steel diaphragm. The diaphragm is scored so that it will burst at the desired pressure - typically this occurs when the driver gas has been compressed by a volumetric ratio of 40-70. A strong shock then propagates down the ST and is reflected at the end wall, creating stagnation conditions for expansion through a hypersonic nozzle into the test section. The test section and dump tank are initially kept under vacuum and are separated from the ST by a secondary mylar diaphragm at the ST-nozzle junction. This diaphragm is vaporized by the incident shock. Test times are limited by the onset of driver gas contamination and are typically of the order of 1-2 ms.

Pressure transducers mounted at points along the shock tube and at the reservoir allow measurements of the shock speed and the stagnation pressure to be made. These are used to determine the freestream conditions in the test section. T5 is capable of producing stagnation pressures of up to $80 \mathrm{MPa}$, and flow speeds are typically in the range $3-6 \mathrm{~km} / \mathrm{s}$, sufficient to reproduce many of the real-gas effects that are present in real hypervelocity flows. Further information regarding the T5 facility may be found in Hornung et al. (1991).

Conditions for the current series of experiments were relatively benign. The test gas was carbon dioxide for all but two shots, with typical stagnation pressures of $\sim 20$ $\mathrm{MPa}$ and stagnation enthalpies of less than $10 \mathrm{MJ} / \mathrm{kg}$. The freestream velocity and density in the test section were typically $3 \mathrm{~km} / \mathrm{s}$ and $0.03 \mathrm{~kg} / \mathrm{m}^{3}$, respectively. A full list of run conditions is given in Appendix B. In all experiments described here, a conical nozzle of half-angle $7^{\circ}$ and with an exit diameter of $300 \mathrm{~mm}$ was used together with a throat of diameter $30 \mathrm{~mm}$. 


\subsubsection{Experimental Setup}

In Figure 5.2 we see a cutaway of the T5 test section with the experimental configuration used in the later technique validation experiments, described in Section 5.3.1, as well as the proximal body experiments, described in Section 5.3.2. The primary sphere, of diameter $63.5 \mathrm{~mm}$ (2 1/2 inches), was rigidly attached to the test section by means of a conical-cylindrical sting and mounting plates. The mounting allowed for adjustments in both the horizontal and vertical position of the primary sphere. Pitot pressure measurements were provided by a pressure transducer mounted in a probe a short distance behind the front point of the sphere.

The secondary sphere, of diameter $31.8 \mathrm{~mm}$ (1 1/4 inch), was positioned directly above and behind the primary sphere. It was suspended from the roof of the test section in such a way that it was able to move freely over the distance it was expected to travel during the test time. A variety of suspension methods were tested and will be discussed shortly. An accelerometer was mounted inside the sphere - a schematic of the model with accelerometer is shown in Figure 5.4. In the experiments described here, a single uniaxial accelerometer was mounted in the drag direction, but if multiple-component force measurements were required, this could be replaced by a triaxial accelerometer. The sphere was constructed so that the geometric center coincided with the center of mass, ensuring that the aerodynamic loading would not result in the generation of any rotational moments. For a general body, however, a combination of accelerometers mounted at different locations within the body could be used to measure both forces and moments.

A catcher, in the form of a bent tube, was mounted behind the suspended sphere. The purpose of the catcher was two-fold. First it served to halt the sphere motion after a short distance, preventing the accumulation of a velocity sufficient to damage the sphere or accelerometer (to lessen the impact, a rubber pad was attached to the front of the catcher). The catcher also provided a path by which the accelerometer cable 
could leave the test section without being exposed to the hostile flow environment.

Several suspension methods for the secondary sphere were tested. Initially the sphere was suspended from thick $(1.25 \mathrm{~mm}$ diameter) wire rope with no catcher to impede the motion. The grooves that may be seen on the inside of each hemisphere in Figure 5.4 are for the clamping of these wires. A rubber mat was attached to the roof of the test section to absorb the impact of the swinging sphere. This method led to the accelerometer cable being torn from the sphere during each shot, however, and the thick wires were found to result in high measured drag values (this will be discussed in Section 5.3.1). Thin piano wires $(0.25 \mathrm{~mm}$ diameter) were also tried in this configuration, but these broke under the aerodynamic loading, resulting in the loss of the accelerometer.

The next suspension method tested was thinner wire rope $(0.7 \mathrm{~mm}$ diameter $)$ secured to the test section both above and below the sphere, as shown in the left photograph of Figure 5.3. The lower wires contained some initial slack, allowing the sphere to move freely over a small distance in the drag direction, but were intended to arrest the sphere's motion before striking the catcher. These wires were also found to break during the course of a typical run however, and again led to excessively high drag values.

The final method tested consisted of suspending the sphere from the test section roof by cotton thread, as shown in the right photograph of Figure 5.3. The intention was that the thread would break at the onset of the flow, allowing for free-floating behaviour during the test time. To ensure the sphere was guided towards the catcher, a thin metal pipe of diameter $4.8 \mathrm{~mm}(3 / 16$ inch) was attached to the back end of the sphere such that it could move freely into the hole in the center of the catcher. This pipe also provided further protection for the accelerometer cable from the flow. One disadvantage of this method is that although the pipe might be expected to have a negligible effect on the drag value, the contribution to the lift in the proximal 


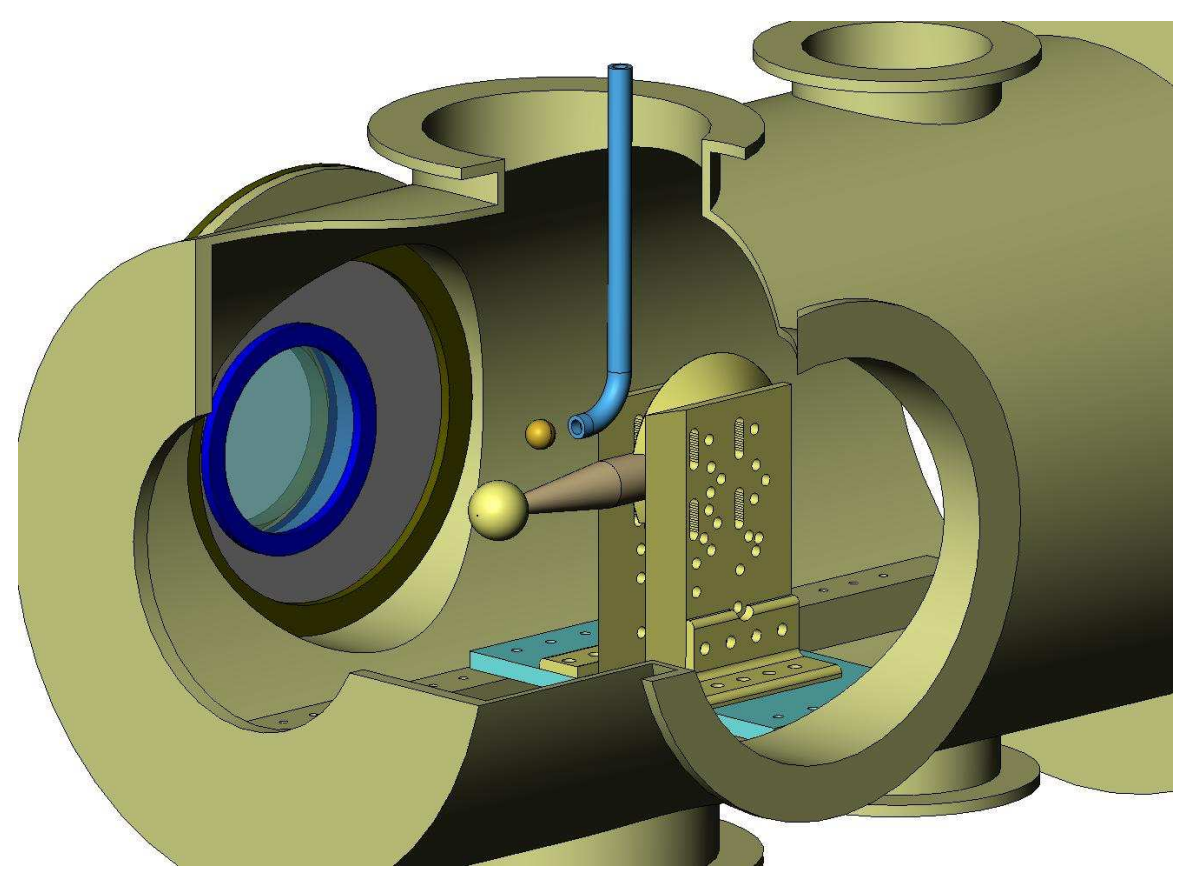

Figure 5.2: Cutaway of the T5 test section showing model arrangement

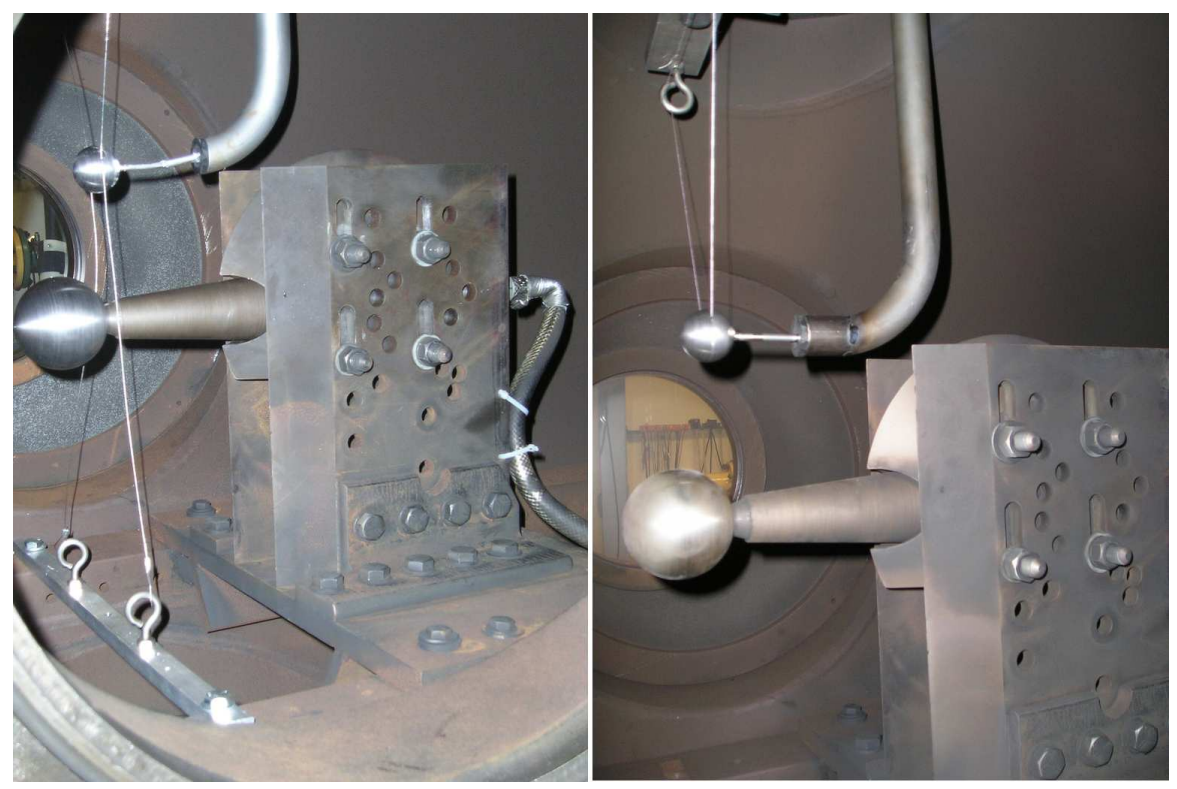

Figure 5.3: Photographs of models in the T5 test section with secondary sphere suspended by wire rope (left) and cotton thread (right). 


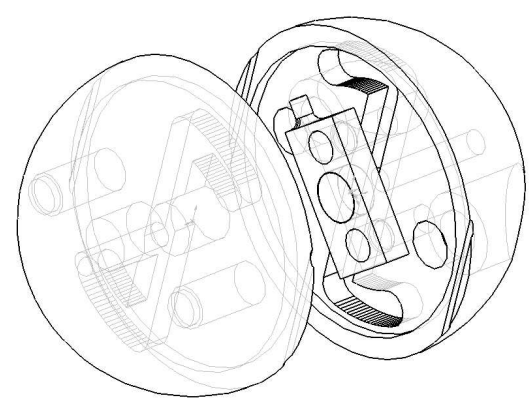

Figure 5.4: Schematic showing an exploded view of the secondary model with accelerometer attached

body experiments would be more significant. Thus, in addition to experiments with accelerometer measurements, shots were also carried out with the accelerometer and metal pipe absent, providing a cleaner configuration. In these shots, the lift and drag were measured only through the displacement profile recorded by a high-speed camera, as will be discussed shortly.

\subsubsection{Accelerometer Measurements}

The primary method of drag measurement was through direct acceleration measurements made by a uniaxial accelerometer mounted inside the model. The accelerometer used was the Endevco model 7270A-2K. This is a piezoresistive-type accelerometer, which allows measurement of steady-state accelerations. This model is rated to accelerations of up to $\pm 2000 \mathrm{~g}$ and has a typical resonant frequency of $90 \mathrm{kHz}$.

In Figure 5.5 the power spectrum and acceleration signal recorded by the accelerometer during a typical shot are shown. Note the log scale on the $y$-axis of the power spectrum plot. Two large peaks are seen in this plot at approximately 85 and $91 \mathrm{kHz}$, respectively, the former of these probably corresponding to the resonant frequency. The main frequency content of the remainder of the signal is well separated from these peaks, which allows them to be removed by the simple application of a 

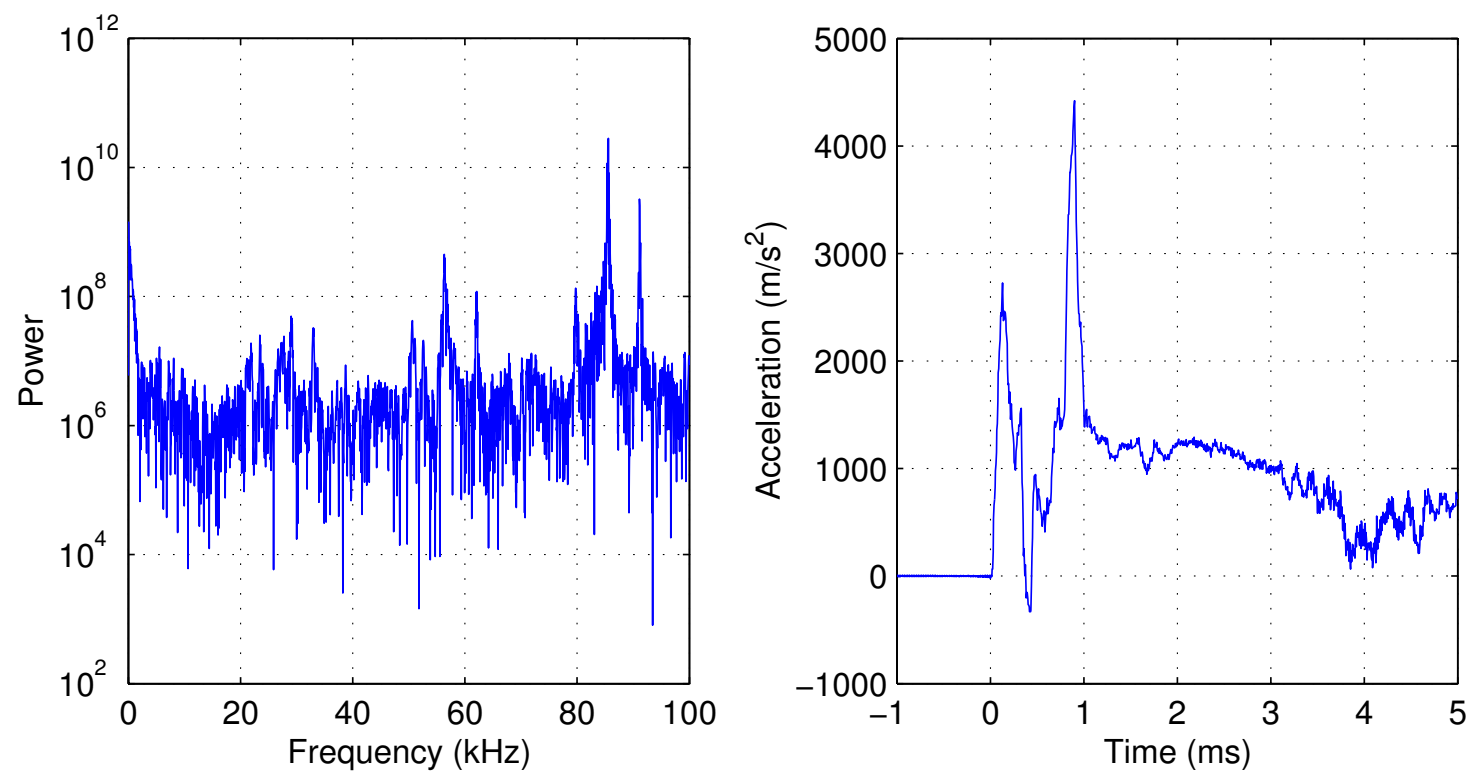

Figure 5.5: (Left) Power spectrum of the accelerometer signal for shot 2322; (right) accelerometer signal after application of low-pass and box-car filters.

low-pass filter. The acceleration signal has been filtered in this way and has been further smoothed with the application of a box-car filter of width 21 time-steps. The steady flow period in this signal is between approximately 1.5 and $2.5 \mathrm{~ms}$, and the mean acceleration is calculated over this time. Given that the mass of the sphere is known, the drag force is then easily calculated.

To determine the coefficient of drag, knowledge of the freestream conditions is required: in particular the density and the velocity. To this end, the reservoir conditions are calculated from the reservoir pressure and shock-timing measurements using ESTC (Equilibrium Shock Tube Calculation) due to Mcintosh (1969). Freestream conditions are then calculated with NENZF (Non-Equilibrium NoZzle Flow) due to Lordi et al. (1966). For the conical nozzle, as the flow continues to diverge past the nozzle exit, the nozzle calculations are continued as far as the downstream coordinate of the sphere in question. 


\subsubsection{High-speed Camera Measurements}

In addition to the accelerometer measurements, a high-speed camera was used to track the motion of the secondary sphere in both the drag and lift directions. The optical setup, a conventional Z-schlieren system, is shown in Figure 5.6. For clarity, the arrangement of the optical components shown has been adjusted slightly from the physical arrangement.

A Vision Research Phantom v5 high-speed digital camera was used in conjunction with a continuous white light source. Three different resolutions were used: $256 \times 256$, $256 \times 128$, and $256 \times 64$ pixels. The frame-rate was set to the maximum allowed by each of these resolutions; respectively 12000, 25000, and 38000 frames/second. The exposure time was typically $10-20 \mu$ s. This was usually short enough to negate the selfluminosity in the test section, although for nitrogen test-gas shots this was more of a problem. For the proximal body experiments, a high pass optical filter was inserted in the optical setup just ahead of the knife-edge to further reduce this luminosity.

The recovery of a time-dependent acceleration profile from displacement measurements is a questionable operation, as it involves the double differentiation of a noisy signal. Thus, the interpretation of unsteady effects using this method will not be attempted here. Assuming, however, that the acceleration is steady over the test time, a second degree polynomial may be fitted to the displacement in a given direction as a function of time. The acceleration in that direction is then simply given by twice the quadratic coefficient.

The acceleration was thus deduced from the camera images in the following way. For each image obtained during the test time, an edge detection was carried out on the entire image using the Sobel method. Points were chosen on the edges corresponding

to the moving sphere, and a circle of the form $\left(x-x_{0}\right)^{2}+\left(y-y_{0}\right)^{2}=r^{2}$ (where $x_{0}, y_{0}$, and $r$ are the unknowns) was fitted in the least-squares sense to these points. While this equation is not in a form to which a standard multiple regression may be 


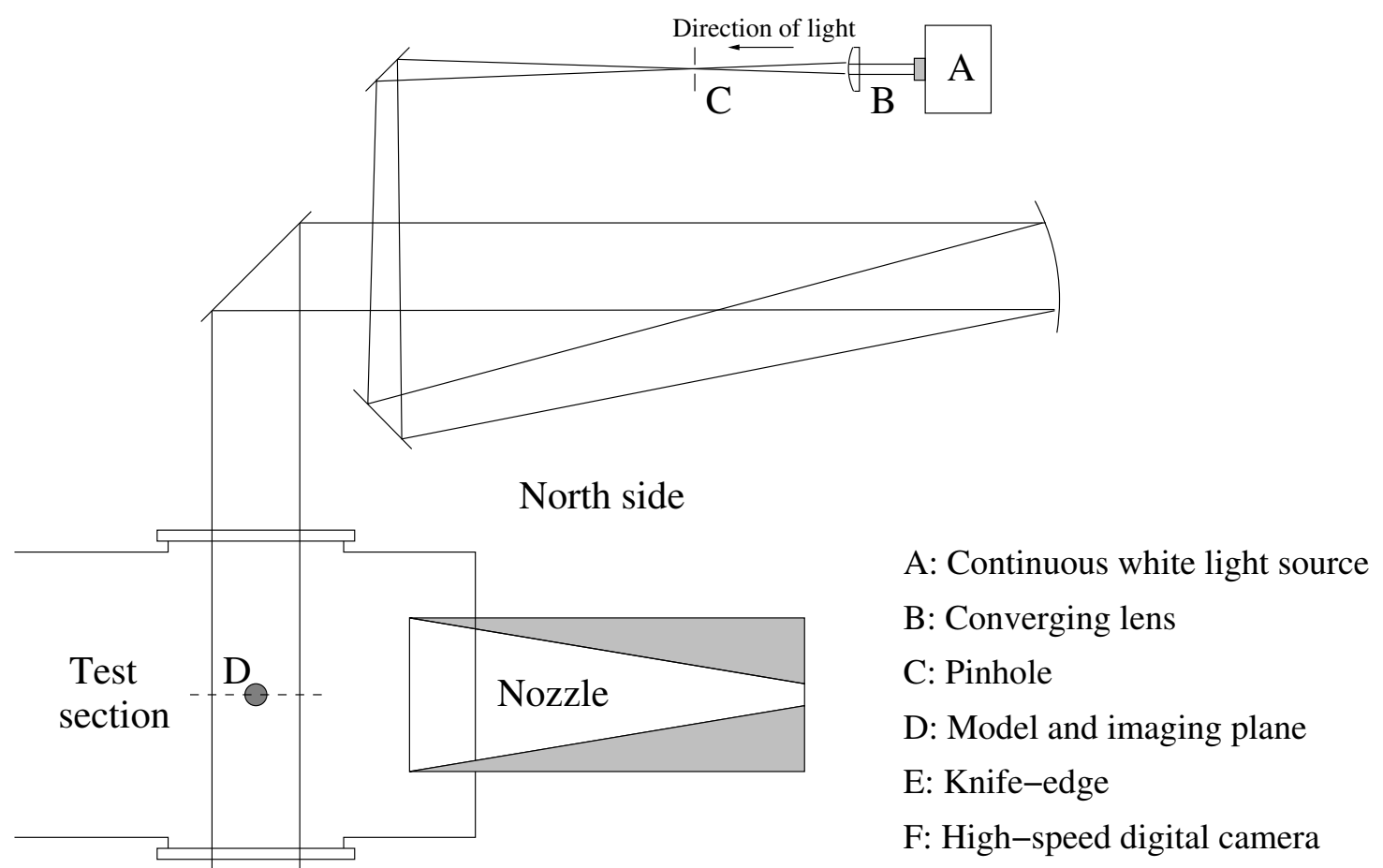

South side 
applied, if we rewrite it as $z=a x+b y+c$ where $z=x^{2}+y^{2}, a=2 x_{0}, b=2 y_{0}$, and $c=r^{2}-x_{0}^{2}-y_{0}^{2}$, we see that standard linear techniques may be used.

Once a displacement history of the sphere has been determined over the time period of interest, quadratic polynomials may be fitted to the $x$ and $y$ profiles as functions of time. As the standard errors in the accelerations obtained are of interest, it is useful here to outline the fitting procedure, following the exposition of Meyer (1975). If $\mathbf{x}$ and $\mathbf{y}$ are the $\mathrm{n} \times 1$ vectors containing the $x$ and $y$ displacements over the time during which the acceleration is assumed to be constant, and $\boldsymbol{t}$ is the vector of corresponding times, we write

$$
\boldsymbol{\alpha}=\left[\begin{array}{ccc}
1 & t_{1} & t_{1}^{2} \\
1 & t_{2} & t_{2}^{2} \\
\cdots & \cdots & \cdots \\
1 & t_{n} & t_{n}^{2}
\end{array}\right]
$$

Then the coefficient vector of the best fit quadratic for the $x$-displacement profile, for example, is

$$
\hat{\mathbf{c}}=\left(\boldsymbol{\alpha}^{T} \boldsymbol{\alpha}\right)^{-1} \boldsymbol{\alpha}^{T} \mathbf{x} .
$$

We have assumed that the weightings are equal for all points in the profile. An unbiased estimate of the scale factor $\sigma^{2}$ may be obtained from the sum of the squared residuals:

$$
\hat{\sigma}^{2}=\frac{(\mathbf{x}-\boldsymbol{\alpha} \hat{\mathbf{c}})^{T}(\mathbf{x}-\boldsymbol{\alpha} \hat{\mathbf{c}})}{n-3} .
$$

An estimate of the covariance matrix of $\hat{\boldsymbol{c}}$ is then given by

$$
[\operatorname{Covar}(\hat{\mathbf{c}})]=\sigma^{2}\left(\boldsymbol{\alpha}^{T} \boldsymbol{\alpha}\right)^{-1}
$$


The acceleration in the $x$-direction is simply $d^{2} x / d t^{2}=2 \hat{c}(3)$, and our estimate of the standard deviation in this value is $\sqrt{[\operatorname{Covar}(\hat{\mathbf{c}})](3,3)}$.

Figure 5.7 shows the results from this process for a typical shot. The top plot shows the displacement profile in space, while the middle and lower plots show the $x$ and $y$ displacements as functions of time, with the best-fit quadratic polynomials plotted during the steady flow time.

Once the $x$ and $y$ accelerations have been calculated in this way, the drag and lift coefficients may be derived in a manner identical to the accelerometer measurement.

\subsection{Results}

\subsubsection{Technique Validation}

Before the techniques described above were used to model the proximal bodies problem, a series of validation experiments was performed. For these experiments, the two spheres were arranged in a configuration as shown in the left image of Figure 5.8, with the secondary sphere positioned in the uniform freestream outside the primary shock. Such a situation (a sphere in a uniform freestream) can easily be simulated nu-

merically, and experimental and computational drag values can then be compared. A number of experiments were carried out in this configuration using a variety of model suspension techniques and protection methods for the accelerometer cable. These are documented in Table 5.1, together with the drag coefficient value(s) obtained for each shot. Schlieren images from three of these shots are shown in Figure 5.8.

To calculate the numerical drag value, simulations of a single sphere in a uniform, inviscid, ideal gas freestream were carried out using AMROC and Amrita. The AMROC simulation was three-dimensional on a base grid of $50 \times 40 \times 40$ with three levels of additional refinement, each of factor two. At the finest level of refinement, the sphere radius corresponded to 32 cells. Other computational parameters were 

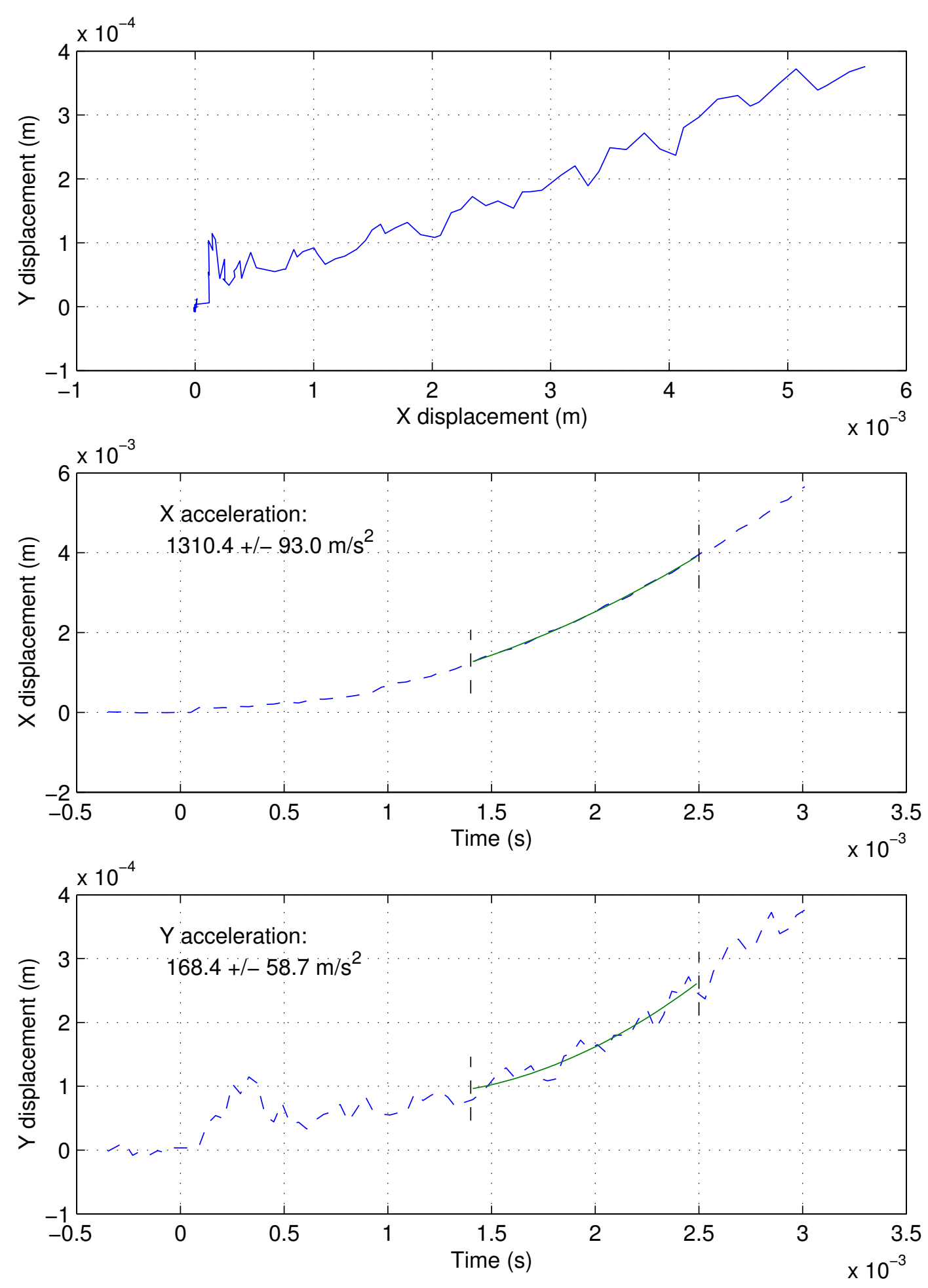

Figure 5.7: Displacement profiles for shot 2330 with best-fit quadratic polynomials: $x-y$ displacement (top), $t$ - $x$ displacement (middle), and $t$ - $y$ displacement (bottom). 


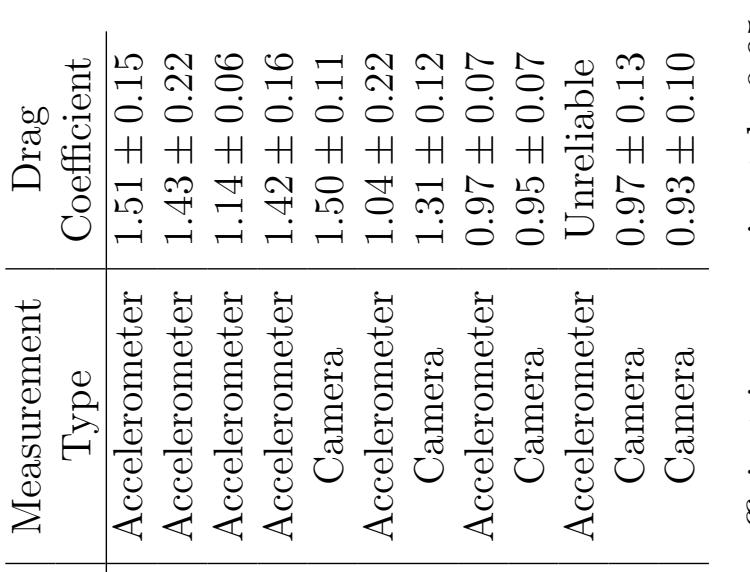

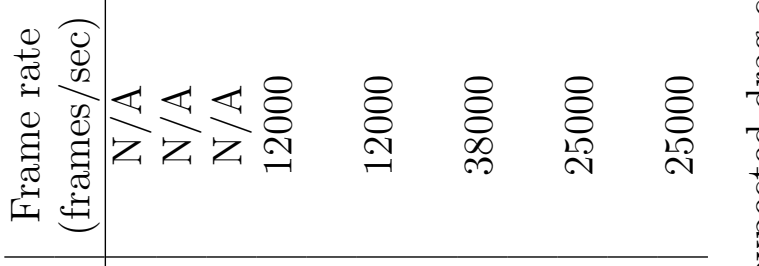

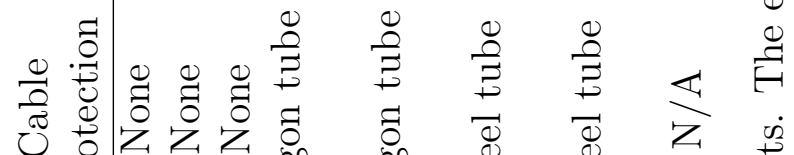
잉

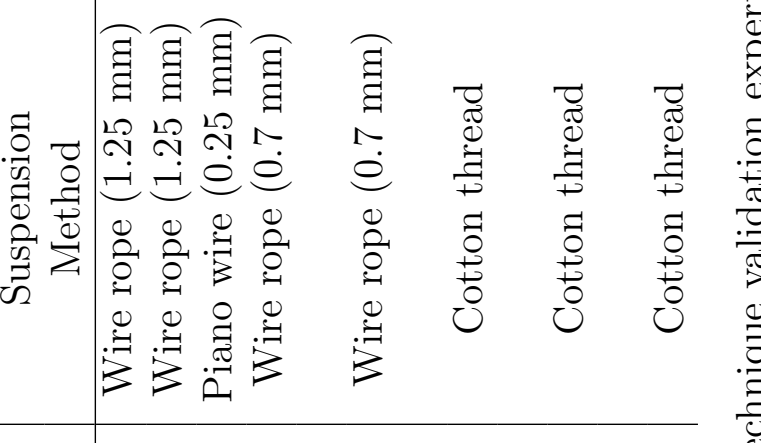

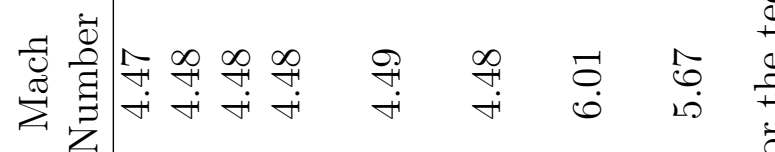

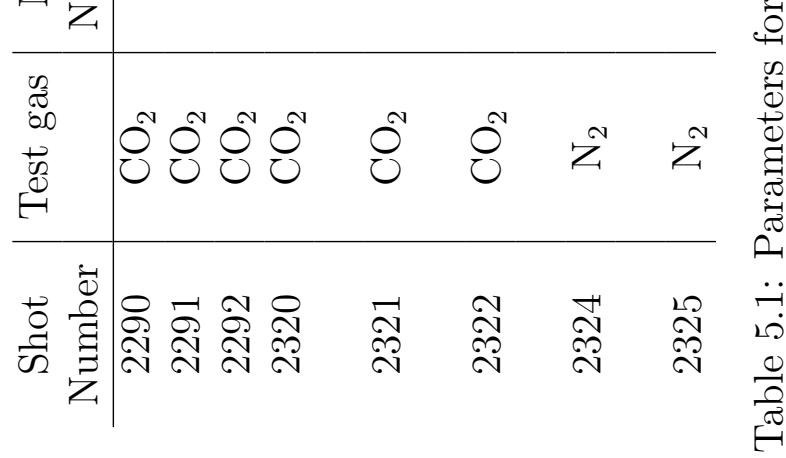



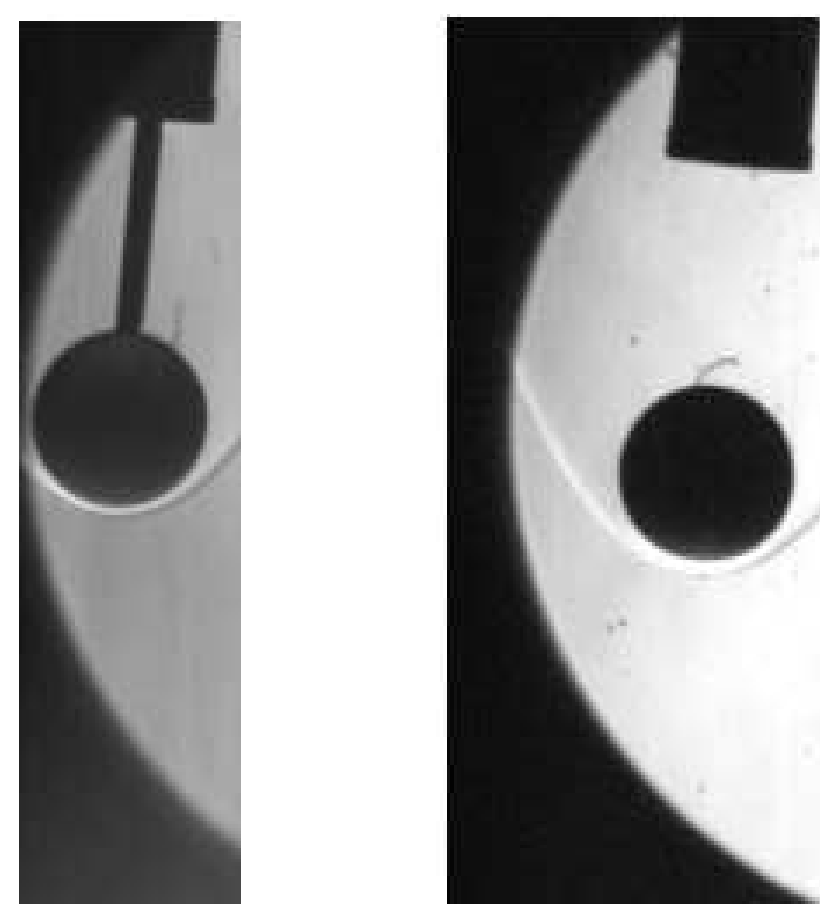

离

$\times$

$\stackrel{2}{2}$

ธี

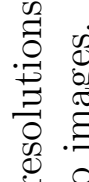

匀

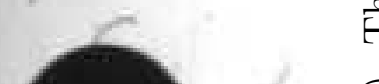

E.

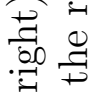

$\dot{0 . \Xi}$

을 웡

농

กㄱ

灵

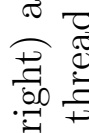

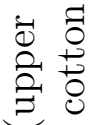

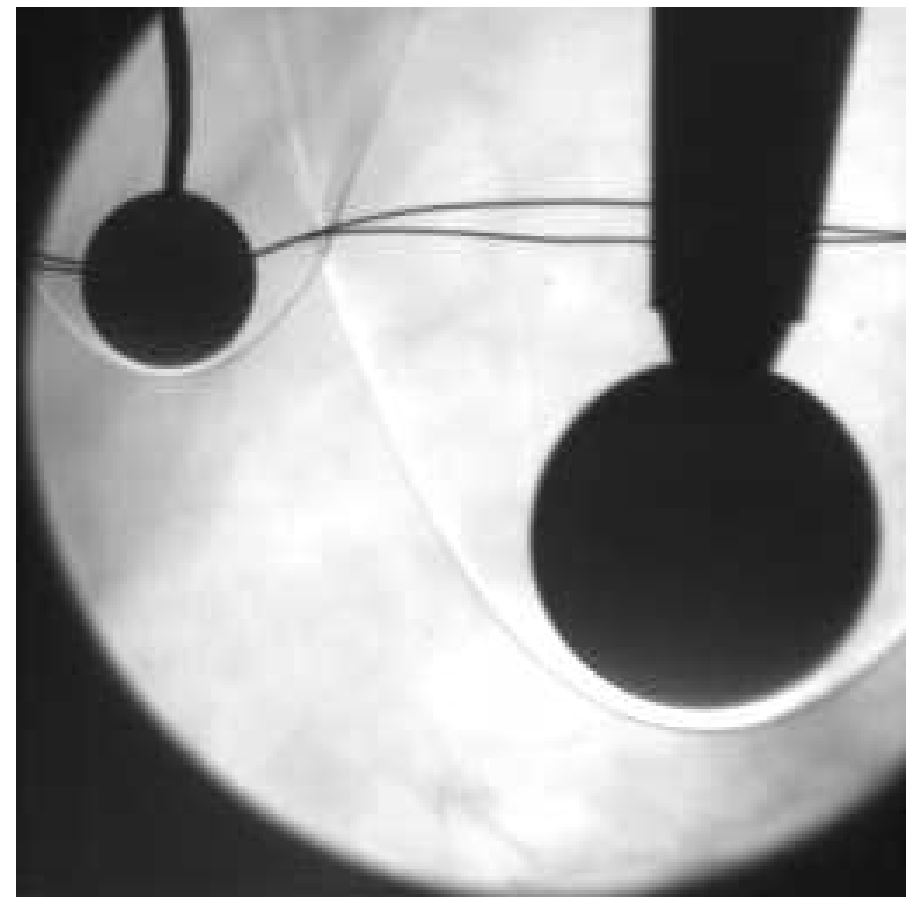

곤

๙ึ

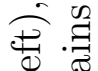

$\stackrel{्}{\circ}$

유

จิ ב

$\frac{3}{2} \frac{1}{0}$

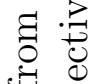

$\sum_{0} 0$

घ्.

- -1

वี $\times$

.0

굴

$\ddot{\infty}$

வ

$\because x$

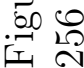


the same as the simulations described in Chapter 3. The Amrita computations were carried out using axisymmetric geometry. The base grid was $320 \times 160$, with one level of additional refinement of factor 2 . One sphere radius corresponded to 80 computational cells at the finest refinement level. Further details of the Amrita computations may be found in Section 5.4.3.

The freestream conditions for the numerical simulations were chosen to be as similar as possible to the conditions experienced by the secondary sphere in the experiments, given the perfect gas assumption. For the $\mathrm{CO}_{2}$ shots, freestream Mach numbers of 4.45 and 4.5 were used for the AMROC and Amrita simulations, respectively. The ratio of specific heats was chosen in the following way. As all $\mathrm{CO}_{2}$ shots were at roughly the same condition, freestream compositions and temperatures (given in Appendix B) varied but very little. The components considered were $\mathrm{CO}_{2}, \mathrm{CO}$, and $\mathrm{O}_{2}$, and values of $\gamma_{i}$ for each at $2000 \mathrm{~K}$ were calculated using the NASA Glenn thermodynamics database tool, Thermobuild (see http://cea.grc.nasa.gov/). The effective ratio of specific heats was then calculated according to the following equation given by Thompson (1988):

$$
\gamma=\frac{\sum_{i} \chi_{i} \frac{\gamma_{i}}{\gamma_{i}-1}}{\sum_{k} \chi_{k} \frac{1}{\gamma_{k}-1}},
$$

where $\chi_{i}$ is the mole fraction of the appropriate component. For the present runs, this gave a value of $\gamma \approx 1.19$. This value was used in both AMROC and Amrita simulations, and drag coefficients of 0.956 and 0.945 were obtained, respectively.

To estimate the effect of viscosity in the $\mathrm{CO}_{2}$ case, a viscous computation was carried out for a perfect gas in axisymmetric geometry by J. Olejniczak. The freestream conditions for this simulation were chosen to be those corresponding to shot number 2322. The calculated drag coefficient was 0.997 , indicating that viscosity contributes approximately $5 \%$ to the drag value. Further details of this computation may be found in Section 5.4.4. 
To simulate the two nitrogen shots, a freestream Mach number of 5.8 and $\gamma=1.35$ were specified. This was a compromise between the two shots, as they had quite different freestream conditions. This value of $\gamma$ is appropriate for molecular nitrogen at a temperature of $900 \mathrm{~K}$ (negligible amounts of atomic $\mathrm{N}$ were present in both shots). AMROC was not used to calculate the nitrogen drag coefficient; the Amrita simulation yielded a value of 0.896 . Although a viscous simulation was not carried out for nitrogen, we would expect viscosity to contribute around $5 \%$ to the drag value here also.

In comparing the experimental drag values of Table 5.1 to the relevant numerical values, the first point to notice is that the experimental values in those shots for which the model was suspended by metal wires are significantly higher than the numerical values. The likely reason for this may be seen in the left image of Figure 5.8, which shows a schlieren image taken during shot 2320. Tension in the wires is obviously present as a result of aerodynamic loading, and a non-negligible component of this is in the drag direction. Oscillations in the wire ropes are also observed during the test time, which could be responsible for the typically higher standard errors in the drag values for these shots. It was for these reasons that cotton thread was adopted as the suspension method.

The drag coefficients obtained in those shots in which cotton thread was used, i.e. shot numbers 2322, 2324 and 2325, show much improved agreement with the numerical coefficients. In each case the appropriate numerical value (whether or not the viscous contribution is included) is within the error bound of the experimental value. We conclude that of the suspension methods tested here, only cotton thread is able to give reliable results. Finally, we note that agreement between accelerometer and camera drag values is generally good, in each case agreeing to within the standard error. 


\subsubsection{Proximal Bodies Experiments}

A limited number of experiments in the proximal bodies configuration were performed - unfortunately, time and financial constraints restricted the extent of this experimental investigation. A combination of high-speed camera and accelerometer measurements were made. In all experiments the test gas was $\mathrm{CO}_{2}$, and the Mach number was approximately 4.5 .

The results from the proximal bodies investigation are shown in Table 5.2. The axial and lateral displacements are calculated from the centers of the two bodies and are given in primary body radii. Also indicated is the position of the primary bow shock relative to the secondary body. The measured drag and lift coefficients are given, together with their standard errors. To obtain these values, the drag and lift forces were normalized by the freestream conditions at a position downstream corresponding to the leading point of the secondary sphere in its initial position. Corresponding inviscid, perfect gas computational values are also indicated. Figures 5.9 through 5.12 show experimental and computational schlieren images obtained for each shot. The experimental images have been rotated to compensate for the rotation in the T5 optical setup. For those shots in which the primary body is not visible in the image, reference images were taken before the shot to determine the relative displacements of the two bodies.

The computational values were obtained from AMROC simulations using the same relative body displacements as the experiments. A specific heats ratio of $\gamma=1.19$ was chosen as outlined above. To simulate the flow produced by the conical nozzle, diverging conical flow was specified at the inlet. The density and velocity for the calculation of the computational force coefficients were obtained by probing the flow solution in the freestream region at a distance downstream corresponding to the leading point of the secondary sphere.

The computational parameters for the numerical simulations were the same as 


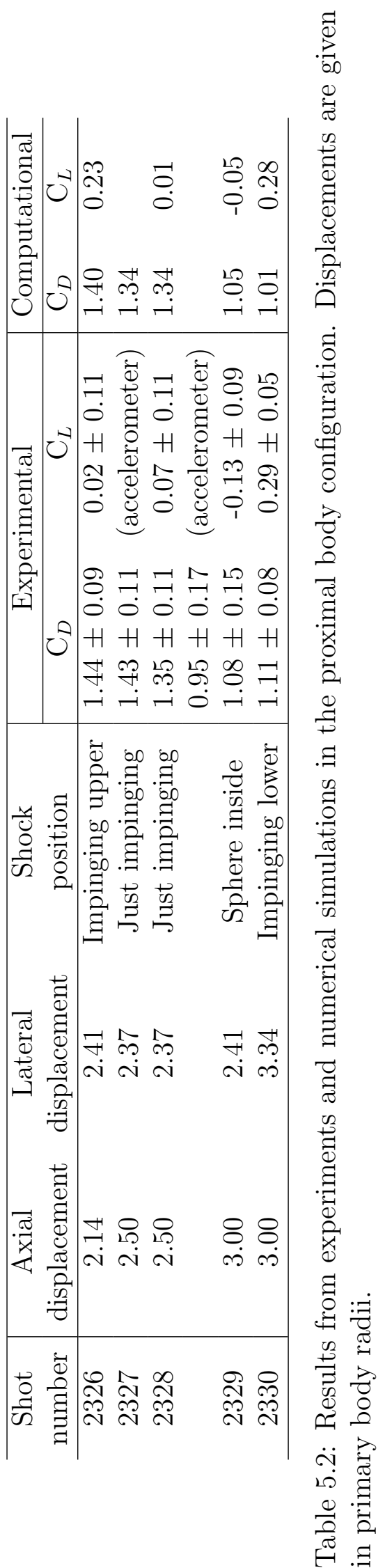


those of the three-dimensional refinement study of Chapter 3. Three additional levels of refinement were used over the base grid, with refinement factors of 3 at the highest level and 2 at the lower two levels. As may be seen in the schlieren images, in all shots the secondary body was relatively close to the primary bow shock. In the refinement study we found that in such a configuration, refinement errors of $\sim 2 \%$ and $\sim 5 \%$ of the drag value could be expected in the computational drag and lift values, respectively, at three levels of refinement, each of factor 2. As the present computations are slightly more refined, we would expect the errors in the computational values to be slightly less here.

The agreement between experimental and computational values seen in Table 5.2 is generally good. The accelerometer signal in shot 2328 was somewhat questionable - on integrating the measured acceleration, displacements significantly less than those recorded in the images were obtained. The signal in shot 2327, in which the configuration was identical, shows much closer agreement with both the computed value and the drag value obtained from the images. It is possible that the accelerometer sustained damage during shot 2327, resulting in the observed discrepancy in the following shot.

Aside from this discrepancy, all experimental values agree with the computational values to within the standard errors, with the exception of the lift measurement in shot 2326 (although the computational drag value in shot 2330 lies outside the experimental error, if we include a computational error of $2 \%$, agreement is obtained .) The computational lift value in shot 2326 is significantly higher than the experimental value, but we should note that in this impinging configuration, the lift value will be very sensitive to any slight differences in the impingement point between the computation and experiment. Note also that the experimental drag values are consistently slightly higher than the computational values - this may be explained by the lack of a viscous drag component in the numerical simulations. 


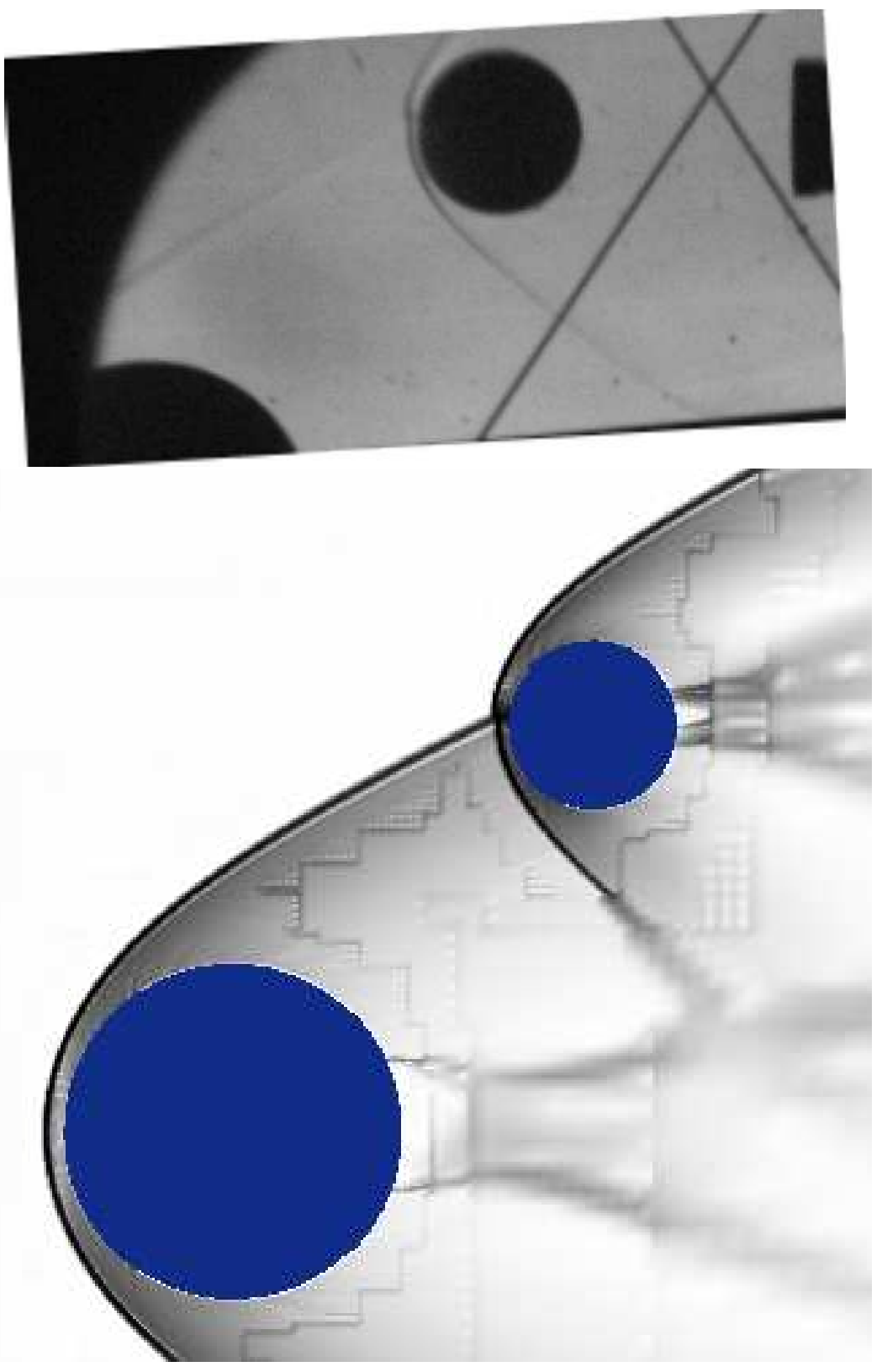

Figure 5.9: Experimental (above) and computational (below) schlieren images of shot 2326. The cross-wire is outside the flow and was used to provide a reference point. 


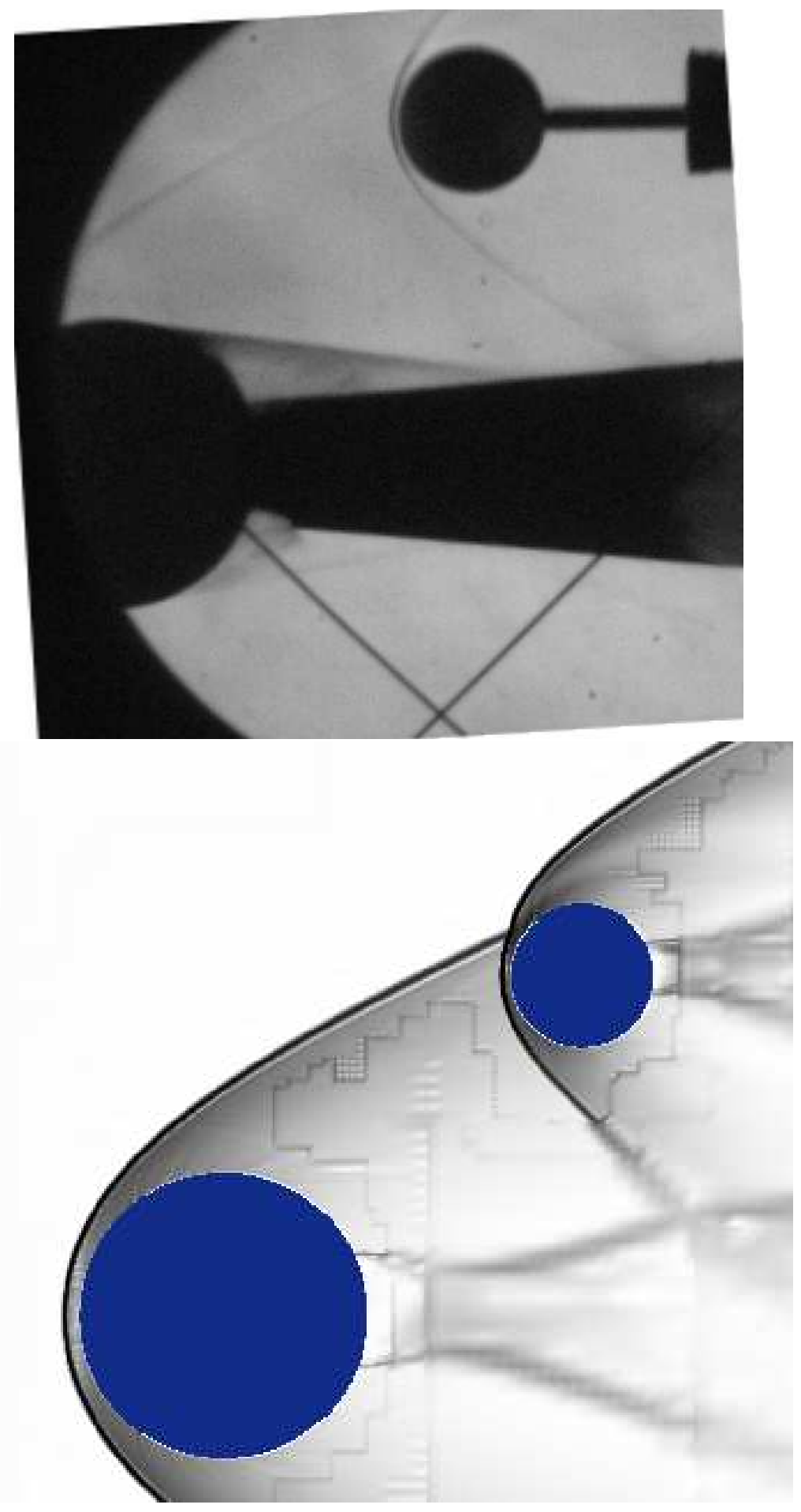

Figure 5.10: Experimental (above) and computational (below) schlieren images of shot 2328. This was also the configuration for shot 2327. 


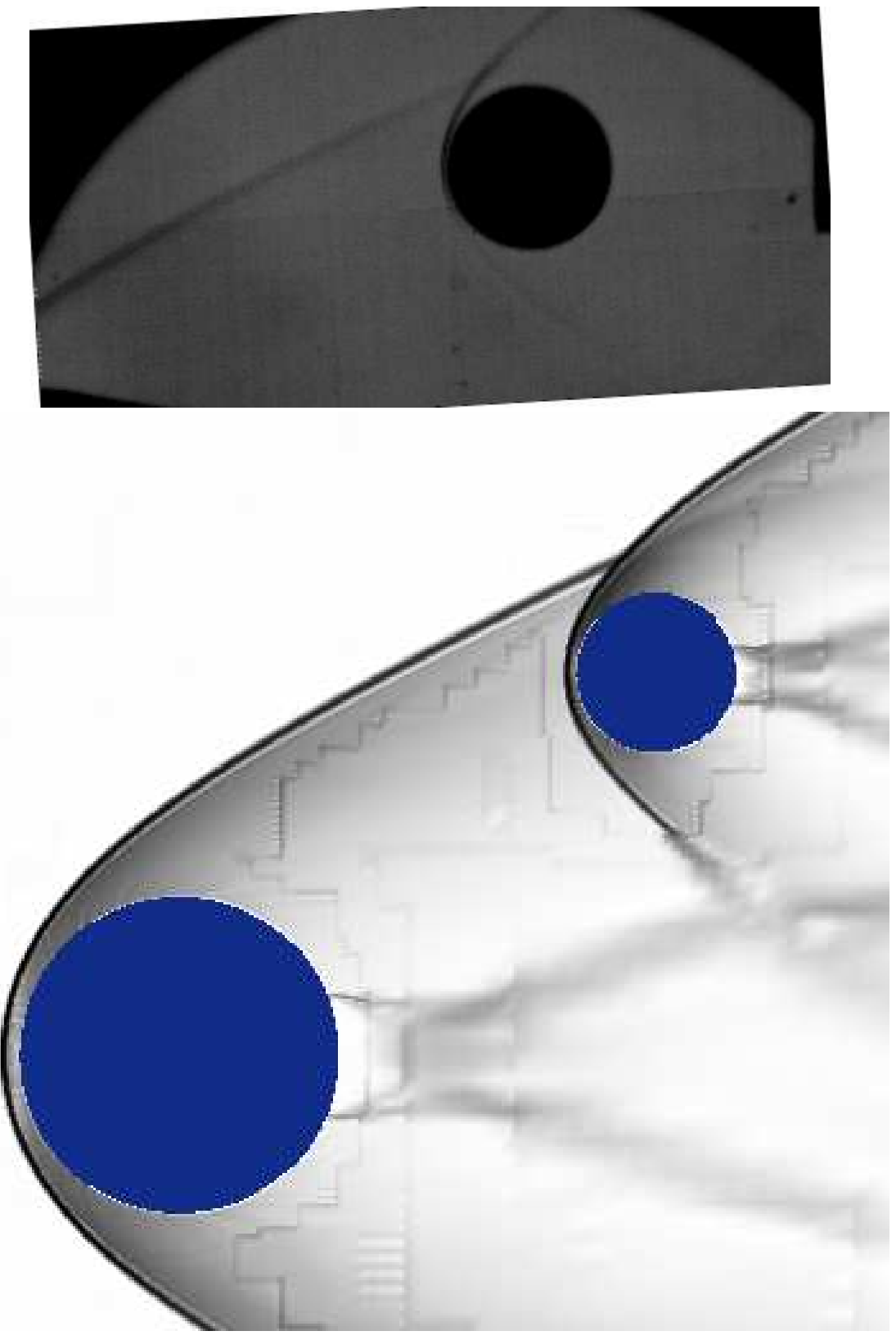

Figure 5.11: Experimental (above) and computational (below) schlieren images of shot 2329 


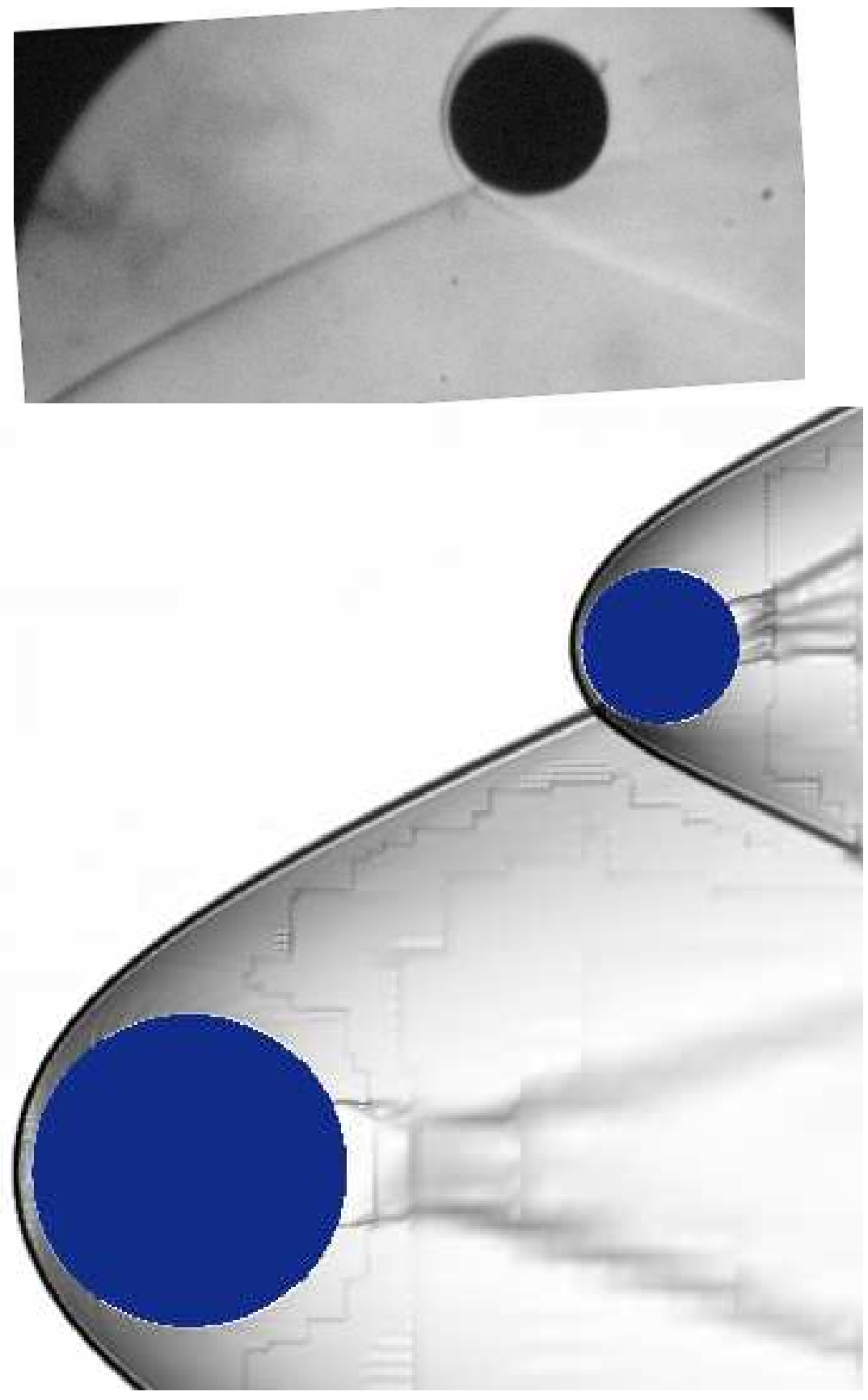

Figure 5.12: Experimental (above) and computational (below) schlieren images of shot 2330 


\subsection{Error Analysis}

There are a number of sources of error associated with the measurements that have been described above. These may be loosely grouped into two categories: those that contribute to the standard error seen in the signals and those that will result in systematic errors. In the first group we have the following:

\subsubsection{Model Movement}

The movement of the secondary sphere during the test time could lead to further errors in two ways. First, if the sphere attains significant velocity during the test time, the freestream velocity will not be an accurate indicator of the flow velocity in the frame of reference of the sphere. However, in Figure 5.13, in which the sphere acceleration, velocity, and displacement during a typical shot are plotted, we see that the maximum sphere velocity during the test time (indicated by the vertical dashed lines) is less than $4 \mathrm{~m} / \mathrm{s}$, a negligible fraction of the freestream velocity. The velocity profile in this plot was calculated by an integration of the accelerometer signal.

The second possible source of error caused by the sphere's movement is the resulting change in the sphere's position during the test time. In Figure 5.13, we see that this change in position is of the order of $2.5 \mathrm{~mm}$, or approximately one sixth of the sphere radius. While for the technique validation experiments this movement will have little effect on the forces experienced, in the proximal body experiments the effect may not be so negligible. In Chapter 3 we noted that in those configurations in which the primary bow shock was impinging on the secondary body, the computed forces were quite sensitive to the location of the impingement point. Thus, in the

proximal body experiments we would expect the forces on the secondary body to vary slightly as the position of the sphere changes over the test time. As we assume constant accelerations, this will contribute to the standard error in the signals. 

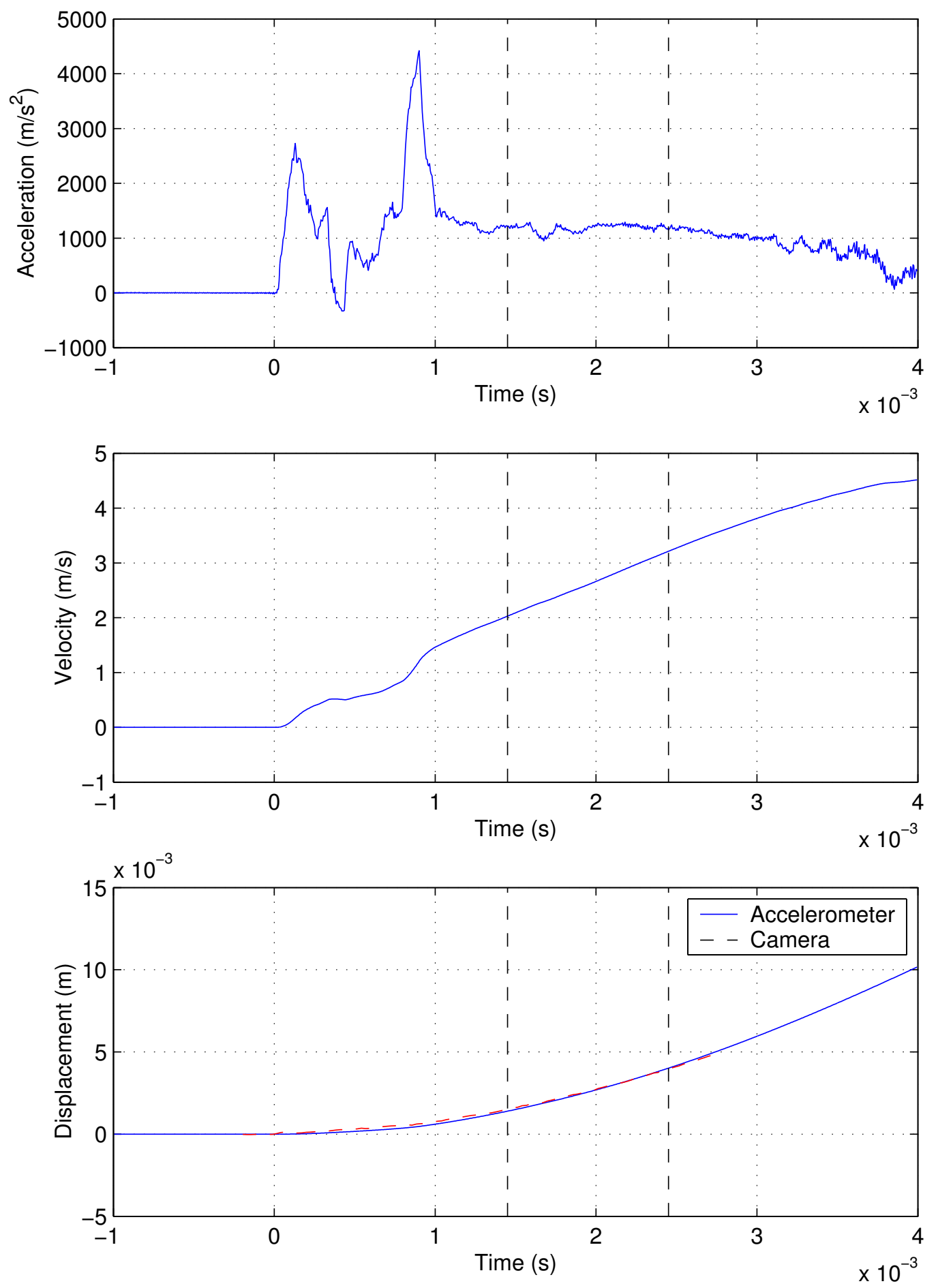

Figure 5.13: Acceleration, velocity, and displacement in the $x$-direction experienced by the suspended sphere in shot 2322 . 


\subsubsection{Flow Unsteadiness}

Compared to flows produced by cold hypersonic facilities, those in the T5 are inherently unsteady. Although we specify a test time over which we assume the flow properties to be constant, this is only an approximation to reality. Assuming that the flow across the conical nozzle is uniform, the unsteadiness recorded in the pitot pressure at the primary sphere will be a good indication of that experienced by the secondary sphere. As the magnitudes of the forces acting on a body are roughly proportional to the pitot pressure, this will also provide a good estimate of the unsteadiness in the force history on the secondary sphere.

In Figure 5.14 are plotted the pitot pressure histories for two shots - the test gas in the upper plot is carbon dioxide; that in the lower plot is nitrogen. The signals have been smoothed using a box-car filter of width 11 to remove noise associated with the transducer rather than the flow. The standard deviation in the mean value over the test time is indicated and is typically $2-3 \%$. In general, the unsteadiness associated with carbon dioxide shots in T5 tends to be less than that for nitrogen shots, as is observed here.

There will also be a secondary effect associated with the flow unsteadiness in the proximal body experiments. In the high-speed camera images, small oscillations in the primary shock position were noted during the test time. This will lead to oscillations in the forces experienced by the secondary body in impinging situations, as the impingement point will be fluctuating in time. The magnitude of these oscillations were, however, typically smaller than the movement of the model during the test time. Thus, the standard error produced by this effect will generally be smaller than that noted in the previous section.

There will also be systematic sources of error, as follows: 


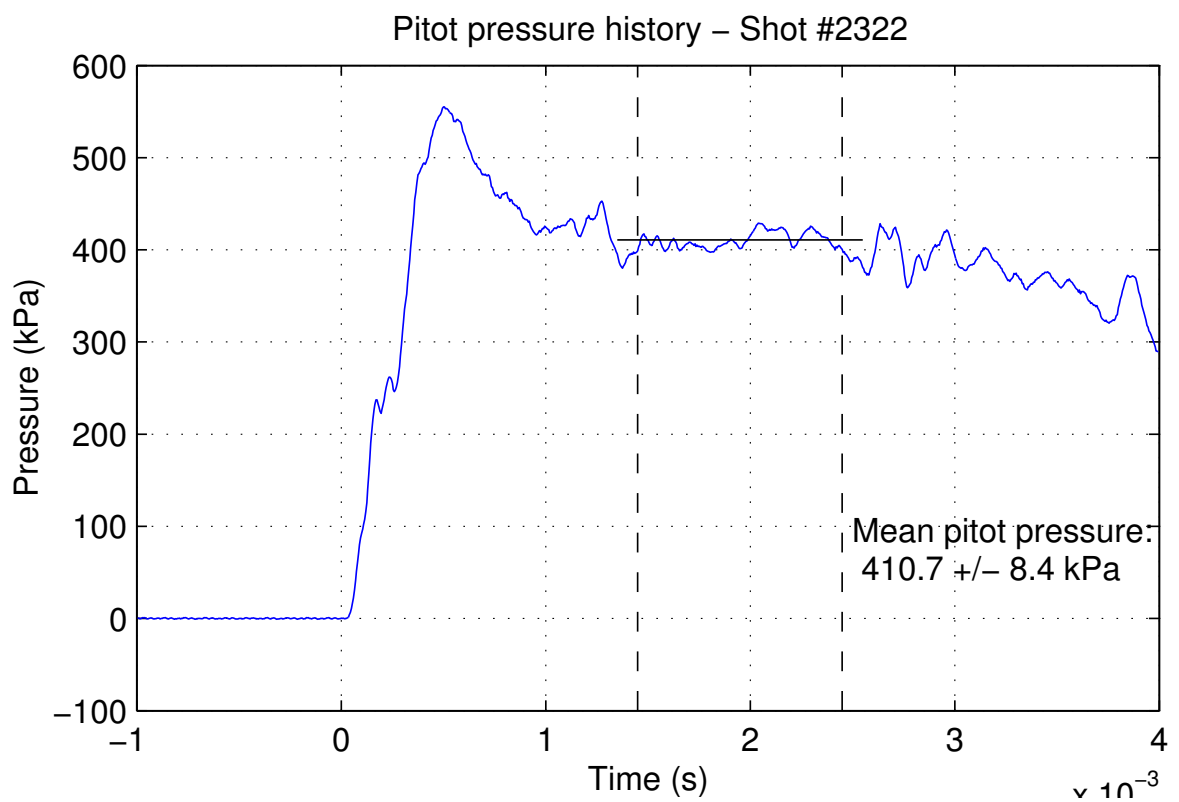

Pitot pressure history - Shot \#2324

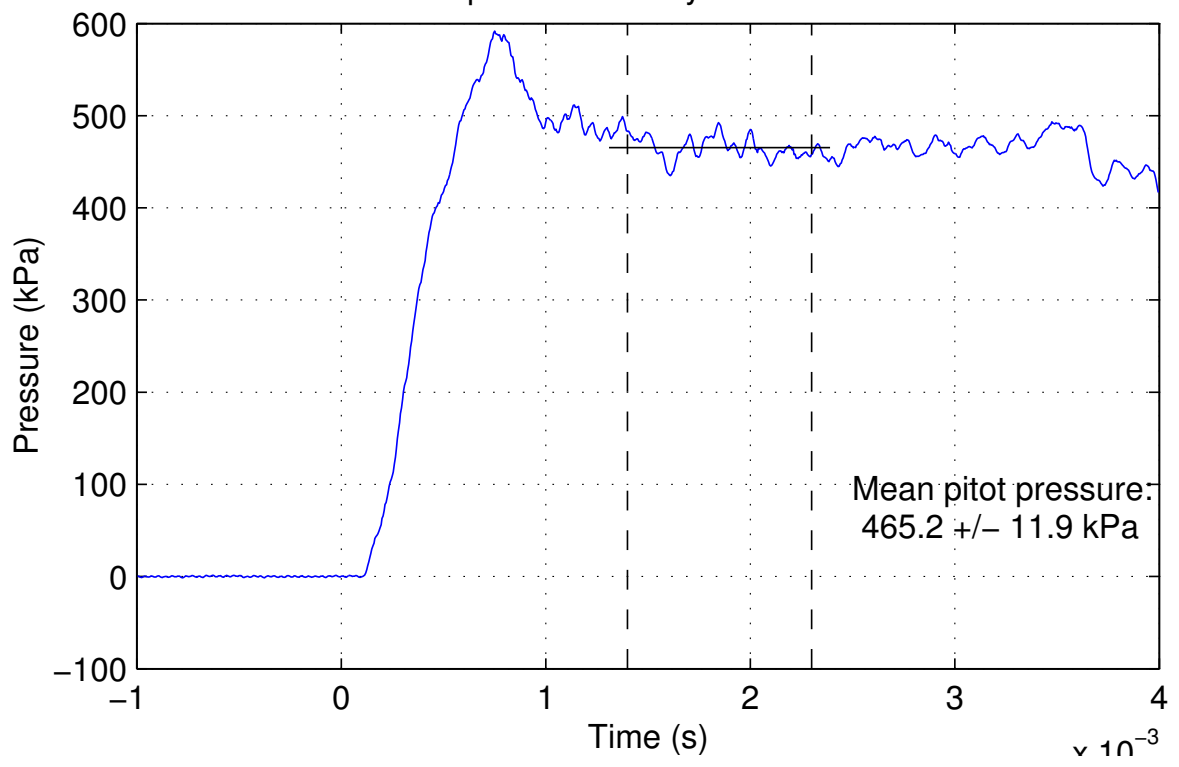

Figure 5.14: Pitot pressure histories for shots 2322 (top) and 2324 (bottom). The test gas in the former is $\mathrm{CO}_{2}$; that in the latter is $\mathrm{N}_{2}$. 


\subsubsection{Effect of the Model Support Components}

The experimental model required support components that were not present in the numerical simulations. We have already noted the effect on the drag coefficient of the wire rope used in the early validation experiments. The use of cotton thread corrected for this, but we might expect the presence of the catcher, and the cable protection tube for those shots in which the accelerometer was present, to affect the flow and thus the measured forces.

To quantify these discrepancies for the technique validation experiments, further numerical simulations were carried out using the Amrita software system in which these physical features were added. Simulations were performed for a ratio of specific heats of $\gamma=1.19$ at a Mach number of 4.5 , simulating a typical $\mathrm{CO}_{2}$ shot, and for $\gamma=1.35$ at a Mach number of 5.8 , to simulate an $\mathrm{N}_{2}$ shot. The simulations were performed on a $320 \times 160$ grid with axisymmetric geometry, with a single level of refinement of refinement factor 2 . The sphere radius was 40 , and the dimensions of the tube and catcher were scaled according to the experimental configuration. Other computational parameters, such as the solver, were the same as those described in Chapter 3.

Simulations were performed for the four permutations involving the catcher and tube. The catcher, in those simulations in which it was present, was positioned 1 sphere diameter behind the back point of the sphere. This was slightly less than the minimum separation of the sphere and catcher during the validation experiments and thus provided a worst-case scenario. The mean drag coefficients on the sphere for these cases are shown in Tables 5.3 and 5.4. Computational schlieren images comparing an unsupported sphere to a sphere with supporting components are shown in Figure 5.15.

We see that the inclusion of the cable protection tube makes a negligible change to the drag coefficient. The catcher has a more significant effect, giving a systematic decrease in drag of between 2 and $3 \%$. The cause of this drag decrease may be seen 
106

\begin{tabular}{c|c|c} 
Tube & Catcher & Mean $C_{D}$ \\
\hline No & No & 0.945 \\
Yes & No & 0.947 \\
No & 1 Diameter & 0.920 \\
Yes & 1 Diameter & 0.924
\end{tabular}

Table 5.3: Calculated drag coefficients for simulations in which model support components are included. The test gas is carbon dioxide, and the Mach number is 4.50.

\begin{tabular}{c|c|c} 
Tube & Catcher & Mean $C_{D}$ \\
\hline No & No & 0.896 \\
Yes & No & 0.898 \\
No & 1 Diameter & 0.876 \\
Yes & 1 Diameter & 0.879
\end{tabular}

Table 5.4: Calculated drag coefficients for simulations in which model support components are included. The test gas is nitrogen, and the Mach number is 5.80.

in Figure 5.15. The presence of the catcher is communicated to the sphere via the subsonic wake, which strengthens the recompression shock and results in a higher pressure on the back of the sphere.

In the proximal body experiments we would expect, if anything, that the effect of the catcher would be smaller, as the wake will be deflected somewhat away from the catcher by the primary shock. We have already noted, however, that this flow deflection is likely to result in the tube having a non-negligible effect on the measured lift.

\subsubsection{Flow Chemistry}

The flows produced by the T5 are at sufficiently high enthalpies that real gas effects become important. We have already noted that in the $\mathrm{CO}_{2}$ shots there are significant fractions of $\mathrm{CO}$ and $\mathrm{O}_{2}$ in the freestream; further dissociation will occur immediately behind the shocks generated by the two spheres. It is a well-known phenomenon that dissociation can lead to a significant increase in density; the effect on pressure 


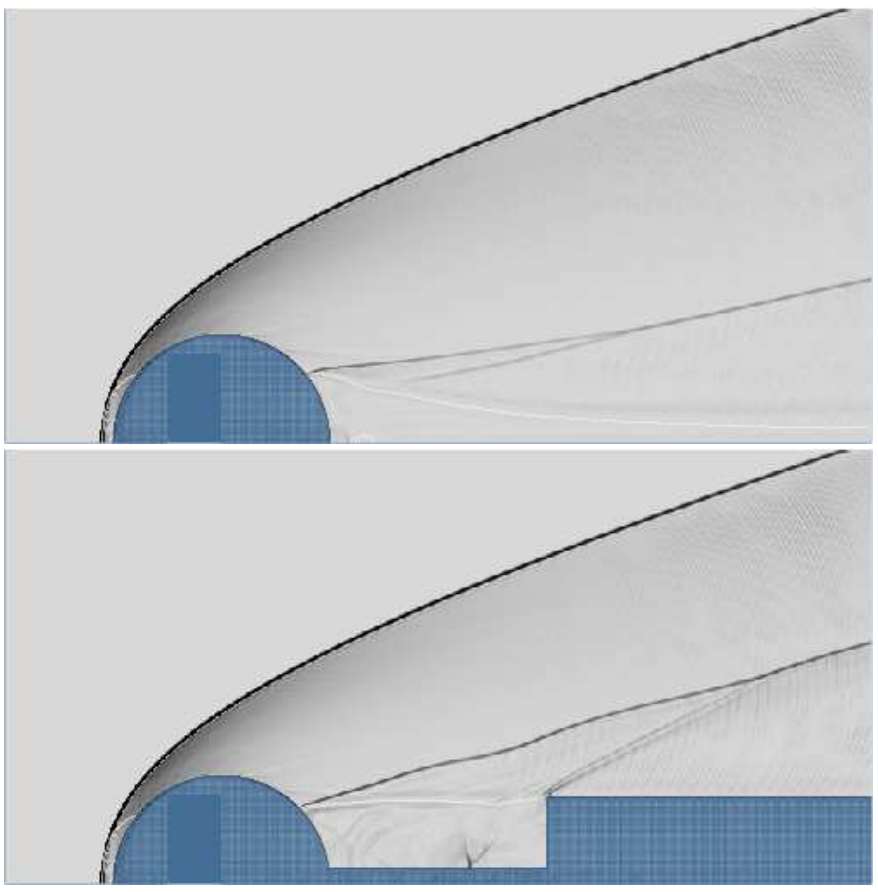

Figure 5.15: Comparison of an unsupported sphere to a sphere with catcher and tube in a carbon dioxide freestream of Mach number 4.5

is typically far less, however. For the full dissociation of a diatomic gas, the increase in pressure behind a normal shock compared to a perfect gas is of the order of $6 \%$ (Hornung, 2002), so we would expect the increase in the current experiments to be even smaller.

In order to estimate the effect of flow chemistry on the measured forces, a pair of simulations were carried out by J. Olejniczak using the DPLR nonequilibrium code developed by Wright et al. (1998). This code solves the equations of motion for thermochemical nonequilibrium flow in both viscous and inviscid cases. Source terms for chemical reactions and vibrational relaxation processes are included and are derived from microscopic considerations.

Simulations were carried out of a sphere in freestream conditions corresponding to those at the secondary sphere in shot 2322 (these are documented in Appendix B). A body-fitted grid with axisymmetric geometry was used, and the effects of viscosity were included in both simulations. Both reacting and perfect gas flows were simulated; 
the drag coefficient and stagnation point pressure were determined in each case. In the perfect gas case, the stagnation pressure was $339600 \mathrm{~Pa}$ and the drag coefficient 0.997; in the reacting case the values were $343300 \mathrm{~Pa}$ and 0.995. These differences are small and could be due to grid artifacts rather than reaction effects. We thus conclude that in the flow regime considered in the current experiments, flow chemistry will affect the measured forces but very little.

\subsubsection{Shot-Induced Structural Vibrations}

During the course of a T5 shot, large changes of momentum occur inside the facility, some of which are transferred to the surrounding structures. This could lead to significant vibrations in the optical setup. These vibrations, if present during the test time, could result in an apparent movement of the secondary body in the camera images. If the vibration frequencies are high compared to the inverse of the test time, this would simply contribute noise to the signal. For longer period vibrations, however, a systematic contribution to the acceleration signal could result.

In an attempt to measure the effect of structural vibrations in the images, a reference point was created by attaching intersecting wires to the outside of one of the test section windows. These may be seen in the experimental schlieren images of Figures 5.9 and 5.10. It is assumed that the T5 test section and dump tank assembly remain stationary during the course of a shot, so any movement recorded in the point of intersection of these wires would be caused by movement of the optical setup. The point of intersection was determined in each image by fitting a straight line to each of the wires.

The $x$ - and $y$-displacements of the intersection point recorded during shots 2326 and 2328 are shown in Figure 5.16. The apparent $x$ - and $y$-acceleration of this reference point during the test time is indicated on each plot (these were calculated in the same way as the sphere accelerations). There does not, however, appear to be any 


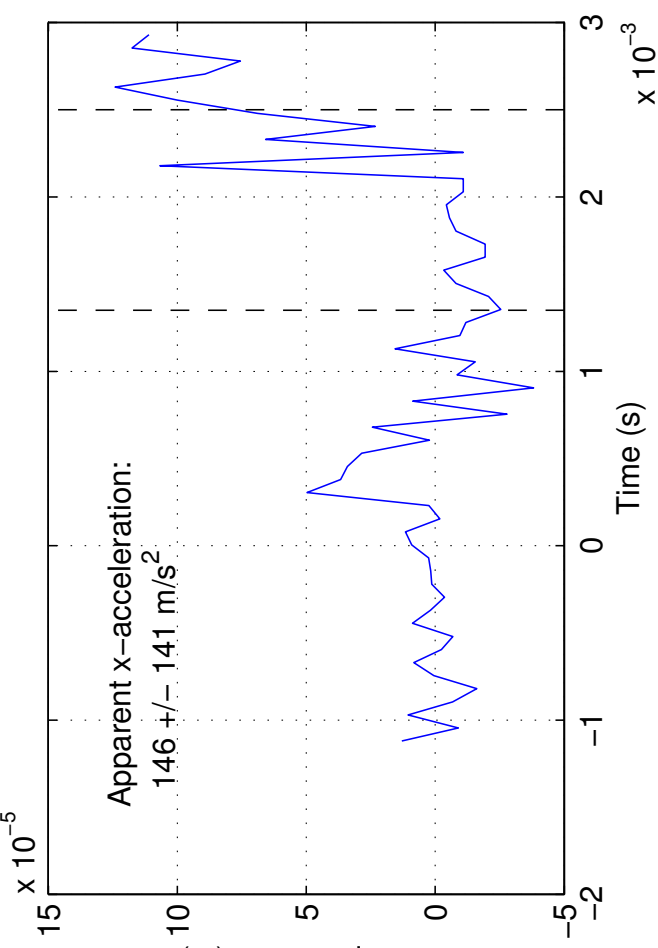

(u) łuәшэе|ds!p $\mathrm{X}$

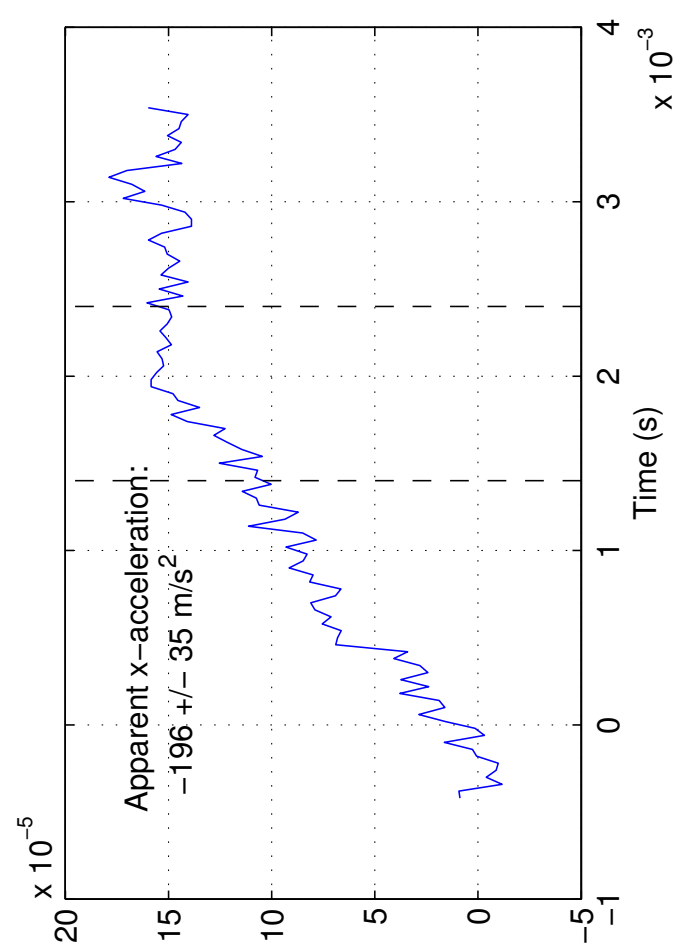

(u) łиәшэе ds!p X

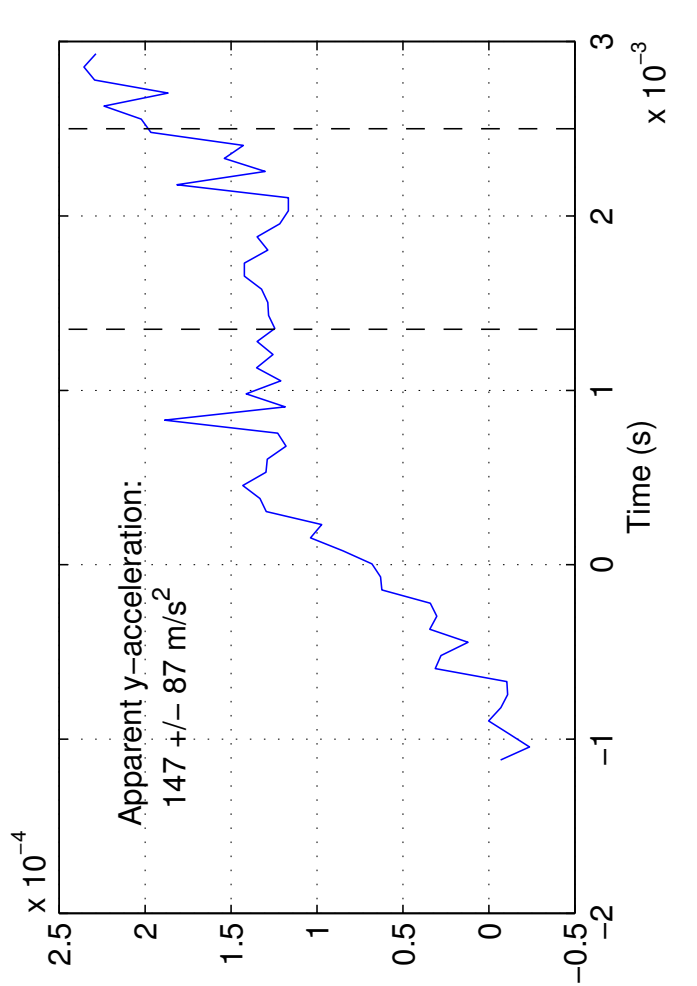

(w) łuəuэe|ds!p ᄉ

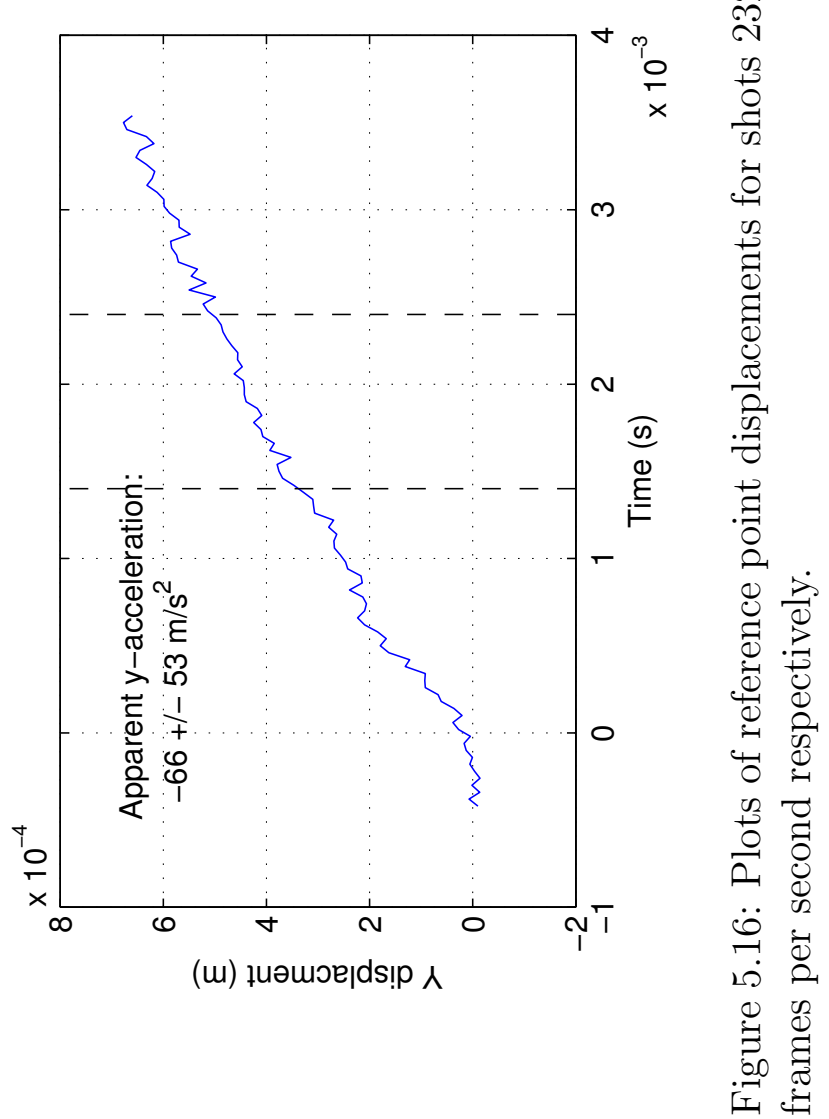


similarity in profiles between the two shots. In shot 2326 there is significant initial movement in the $x$ direction that levels off during the test time, resulting in a significant apparent acceleration. This apparent acceleration is approximately $13 \%$ of the $x$-acceleration recorded by the sphere during the same shot. The $y$-displacement in this shot shows significant movement throughout the recorded time, but the profile is almost linear, resulting in a smaller apparent acceration in this direction. The noise seen in these signals is most likely due to errors in the determination of the reference point, rather than high-frequency vibrations - unfortunately, the fitting of an open curve such as a line results in a larger error than fitting a closed curve such as a sphere.

During the test time of shot 2328 a large jump is seen in both profiles, resulting in large apparent accelerations, but with significant uncertainty. If this jump were due to vibrations in the optics, we would expect it to be present in the sphere displacement profile for this shot. An inspection of this profile did not indicate the presence of such a large jump, however. It is possible, given the small displacements involved, that our assumption that the test section remains stationary is mistaken. The results obtained here do suggest, however, that a further investigation of the effect of structural vibrations would be worthwhile.

\subsubsection{Misalignment of the Model}

While a sphere has no preferred direction, and the orientation of the sphere is thus unimportant for measurements made with the camera, misalignment would affect the accelerometer measurements. The effect of model misalignment would be to slightly decrease the drag value recorded by the accelerometer. If $a_{x}$ is the actual acceleration experienced by the sphere in the $x$-direction, $a_{x}^{\prime}$ is the measured acceleration, and $\theta$ and $\phi$ are the yaw and pitch misalignments, respectively, then $a_{x}^{\prime}=a_{x} \cos \theta \cos \phi$.

Yaw alignment was provided by the suspension arrangement: the suspension 
strings were secured in a $\mathrm{V}$ configuration near the outside points of the sphere by clamping the two hemispheres together over the strings. Correct pitch orientation was to be ensured by the sphere design - in particular by ensuring that the center of mass lay at the geometric center. The inclusion of the metal tube as well as tension in the accelerometer cable will affect the model orientation, however, particularly the pitch.

Fortunately, any pitch misalignment could be measured in the cases in which the metal tube was present. The tube edges provide a reference direction in the images which could be compared to that provided by the nozzle exit. Straight lines were fitted (in the least-squares sense) to the tube and the nozzle exit, and the angle between them was calculated. The maximum deviation from perpendicularity in these shots was $1.2^{\circ}$. The resulting error in the drag from this maximum deviation is $0.02 \%$, so we can safely conclude that the misalignment error is negligible.

\subsection{Movement of the Primary Sphere}

Finally, we make note of a rather unexpected result. During those technique validation shots in which the primary sphere was visible in the camera images, this sphere was also observed to move during the course of the test time. The primary motion was tracked in the same way as the secondary motion, and this allowed for the resolution of displacements of fractions of a millimeter. In Figure 5.17 the movement of the primary sphere in the flow direction is plotted along with the pitot pressure recorded during shot 2321. As may be seen, the profiles are quite similar, with even some of the minor features in the pitot pressure history also present in the displacement profile. The force on the sphere is roughly proportional to the pitot pressure, suggesting that the sphere is responding elastically to the induced aerodynamic loading. While this is not surprising in itself, the apparent shortness of the response time and the ability 
to resolve the movement were somewhat unexpected.

This response of the primary sphere suggested that an alternative force-measurement technique might be possible. In particular, if the movement of the primary sphere could somehow be calibrated, the displacement recorded during a shot would give a direct measurement of the induced force. Such a calibration was attempted with the use of an impulse hammer. Unfortunately, the extent of movement produced by the hammer impact was insufficient to allow a satisfactory calibration to be performed. This is perhaps another area in which further investigation could be warranted.

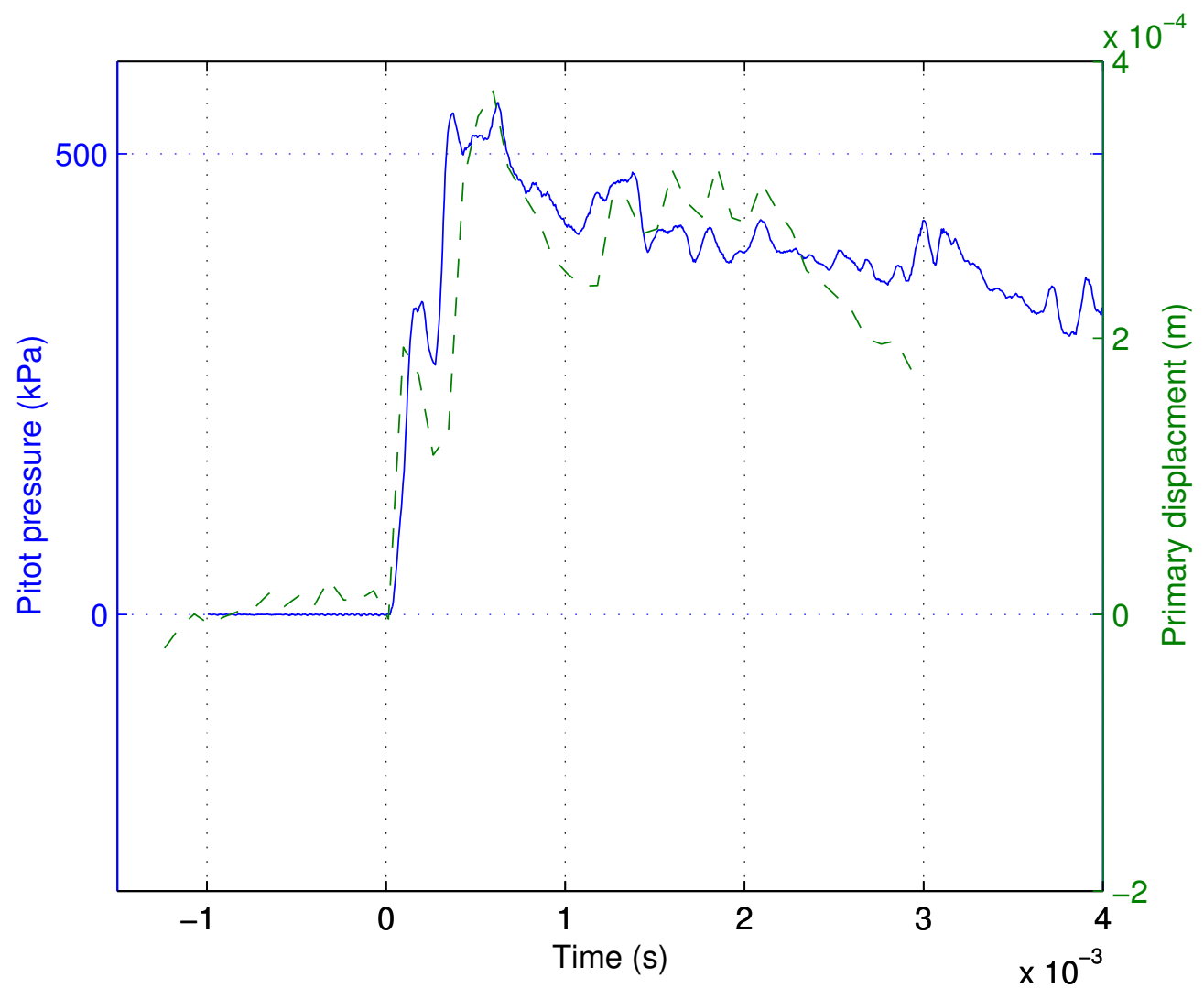

Figure 5.17: Plots of pitot pressure (solid line) and primary sphere displacement in the flow direction (dashed line) recorded during shot 2321 


\section{Chapter 6}

\section{Binary Asteroids in a Planetary Atmosphere}

\subsection{Introduction}

Recent observations have revealed that approximately $16 \%$ of asteroids in near-Earth orbits may be binary systems (Margot et al., 2002). Range-Doppler imaging has indicated that the primary bodies in these systems are typically spheroidal and are spinning at rotation rates near the breakup point for strengthless bodies. Such observations suggest that these bodies are gravitationally bound "rubble-piles". Numerical simulations have indicated that a likely mechanism for the formation of such binary systems is the tidal disruption of a single strengthless body during a close planetary encounter (Bottke and Melosh, 1996).

Evidence for binary systems entering planetary atmospheres may be seen in binary crater fields on Earth and Venus (Melosh and Stansberry, 1991). If the secondary body lies within the primary shock wave generated during the atmospheric transit, we would expect the aerodynamic interactions to affect the final crater locations. These interactions will be especially important in the case of Venus, with its extremely dense and diffuse atmosphere.

The analytic methodology developed in Chapter 2 will allow us to model the aerodynamic interactions during such an entry. Before attempting this, however, two 
tasks await us. First, we must determine the extent of the flow region over which the blast wave methodology may be assumed to give a reasonable solution. Second, the methodology must be extended to include, in particular, the case in which the primary shock impinges on the secondary body.

\subsection{Domain of Validity of the Blast Wave Method- ology}

In Chapter 2, the force coefficients of the secondary body predicted by the blast wave methodology showed good agreement with coefficients obtained through numerical simulations of flows with Mach numbers of the order of 50. This agreement held over a range of secondary body sizes and for downstream displacements taken from the centers of the two bodies of 3 and 8 primary body radii. These correspond to $x / d_{1}$ values of $\approx 2$ and 4.5 , given that the shock stand-off distance is very small at these Mach numbers. There was one important caveat to these results, however, in that the theoretical shock radius was somewhat smaller than the computed radius. As we wish to use the theoretical shock shape in our asteroid simulations, we need to determine if it gives a reasonable approximation to the physical shock shape (as represented by the computed profile).

In the top image of Figure 6.1 is a computational schlieren image of a sphere in a freestream of Mach number 50. This image was produced by an Amrita simulation in which the computational domain included up to 32 body diameters downstream. Also plotted on this image is the shock radius given by the blast wave analogy. As may be seen, the discrepancy noted earlier between the computational and theoretical shock profiles persists downstream, but becomes a decreasing fraction of the shock radius. In particular, the shock angles seem to agree very well to this point downstream. Note, however, that the angles will eventually diverge, as the computational angle 
must tend towards the Mach angle $\beta=\arcsin (1 / M)$, whereas the theoretical angle will tend to zero as $x / d_{1} \rightarrow \infty$. From this picture, then, we conclude that although for small distances downstream there will be a fairly large error in the shock position, the blast wave analogy gives a reasonable approximation to the physical shock shape at these Mach numbers.

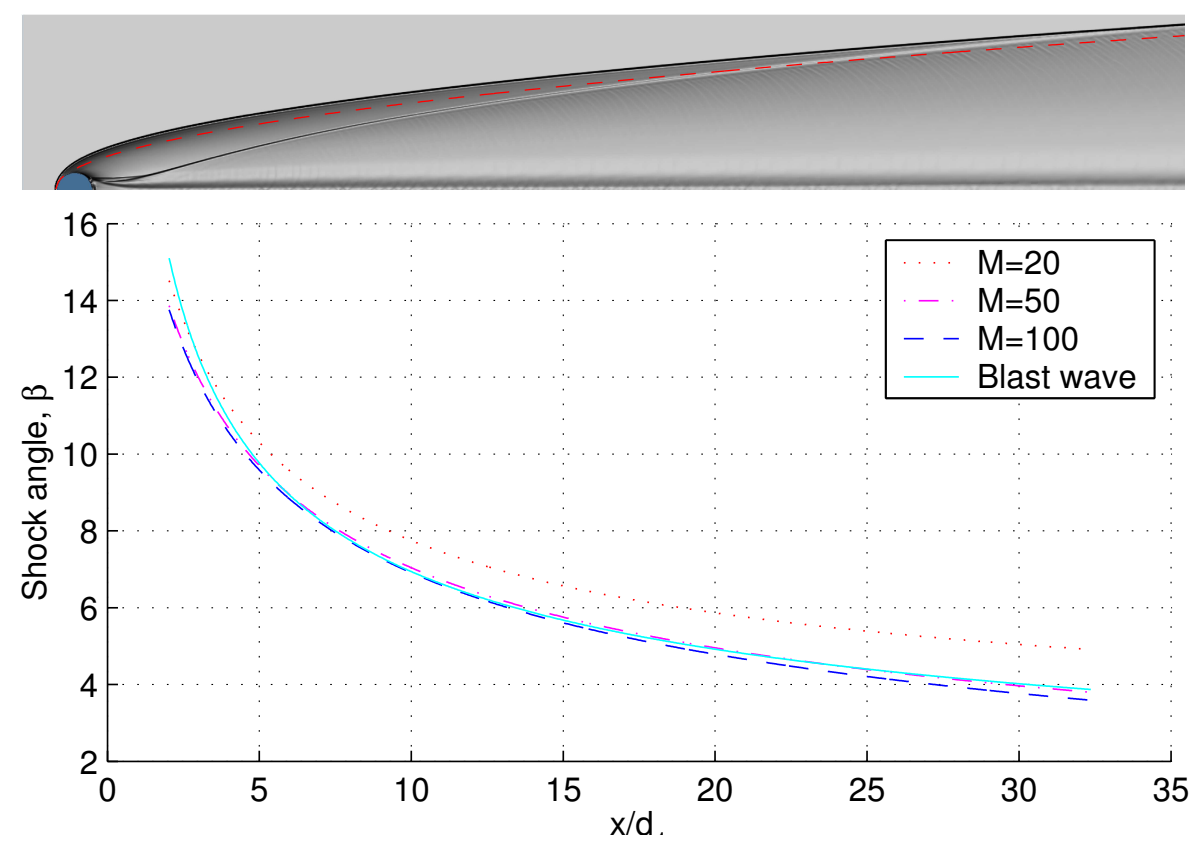

Figure 6.1: Above: The shock wave generated by a sphere in an $M=50$ freestream, with the dashed line indicating the shock shape predicted by the blast wave analogy. Below: Shock angles given by the blast wave analogy and computations at several Mach numbers.

Also plotted in Figure 6.1 are the shock angles as functions of distance downstream for the blast wave analogy and several computations, such as the one described above. Profiles for Mach numbers of 20, 50, and 100 are included. To calculate the shock angle in each case, a general hyperbolic curve of the form $y^{2}=a-b\left(x-x_{0}\right)^{2}$ was fitted to the locus of points given by the location of the maximum density gradient at each point downstream. A curve of this form provides an extremely good fit over the domain shown and allows the shock angle to be easily calculated. The blast wave profile does not give a particularly good match to the $M=20$ curve, but matches the 
other two curves well. The blast wave and $M=50$ curves are all but indistinguishable for $x / d_{1} \geq 5$ : somewhat surprisingly this match is better than that for $M=100$.

In Section 4.3, we raised the possibility of defining an effective origin to compensate for the offset of the blast wave shock relative to the physical shock in the intermediate- and far-fields. The close agreement between theoretical and computational shock angles observed in Figure 6.1 at high Mach numbers provides support for this idea. For each of the computations shown, the mean offset between the computational shock and the blast wave shock was calculated from a distance two body diameters downstream of the stagnation point. For $M=20$, 50, and 100 the mean offsets were $0.46,0.28$, and $0.24 d_{1}$, respectively. Using these offsets, the maximum discrepancy between the blast wave shock and the computational shock over the domain considered were $17 \%, 4 \%$, and $2 \%$. Thus, we conclude that for high Mach numbers $(M \gtrsim 50)$, if we define an effective origin for the blast wave shock with an offset of approximately half a body radius, this will give much improved agreement with the actual shock shape in the intermediate- and far-fields.

Using the fitted hyperbolic computational shocks, we are able to make further comparisons between computational and theoretical predictions. In particular, for a perfect gas, knowledge of the shock angle at a particular Mach number allows us to calculate the flow conditions immediately behind the shock through the oblique shock relations. These may then be compared with the equivalent quantities given by the blast wave analogy. Agreement or otherwise of these conditions will give us an indication of the extent of the flow field over which we may consider the blast wave methodology to give us reasonable results. The most important variables for our calculation of $C_{D}$ and $C_{L}$ are the normal-shock-processed stagnation pressure coefficient $p_{\text {stag }}^{\prime}$ and the flow angle $\delta$, so these shall be considered in that which follows.

We first consider the blast wave case. Let the subscripts $\infty$ and 1 denote con- 
ditions in the freestream and immediately behind the shock wave (i.e., at $r=R_{s}$ ), respectively. The subscript 2 indicates conditions immediately behind a normal shock occurring at condition 1. A primed pressure variable again indicates normalisation by $\rho_{\infty} V^{2} / 2$. From Section 2.4, we have the following:

$$
\begin{aligned}
M_{1}^{2} & =\frac{2}{\gamma(\gamma-1)}\left[1+(\gamma+1)^{2}\left(\frac{x}{R_{s}}\right)^{2}\right] \\
& =\frac{2}{\gamma(\gamma-1)}\left[1+\frac{(\gamma+1)^{2}}{\eta_{0}^{2} \sqrt{\pi C_{D} / 8}} \frac{x}{d_{1}}\right] \\
M_{2}^{2} & =\frac{(\gamma-1) M_{1}^{2}+2}{2 \gamma M_{1}^{2}-(\gamma-1)} \\
p_{1}^{\prime} & =\frac{1}{\gamma+1}\left(\frac{R_{s}}{x}\right)^{2} \\
& =\frac{\eta_{0}^{2}}{\gamma+1}\left(\frac{\pi C_{D}}{8}\right)^{1 / 2} \frac{d_{1}}{x} \\
p_{2}^{\prime} & =p_{1}^{\prime} \frac{p_{2}}{p_{1}} \\
& =p_{1}^{\prime}\left(1+\frac{2 \gamma}{\gamma+1}\left(M_{1}^{2}-1\right)\right) \\
p_{\text {stag }}^{\prime} & =p_{2}^{\prime} \frac{p_{\text {stag }}}{p_{2}} \\
& p_{2}^{\prime}\left(1+\frac{\gamma-1}{2} M_{2}^{2}\right)^{\frac{\gamma}{\gamma-1}} .
\end{aligned}
$$

Note that as $x / d_{1} \rightarrow \infty, p_{\text {stag }}^{\prime}$ tends to a constant value, given by

$$
p_{\text {stag }}^{\prime}\left(x / d_{1} \rightarrow \infty\right)=\frac{4}{\gamma-1}\left(1+\frac{(\gamma-1)^{2}}{4 \gamma}\right)^{\frac{\gamma}{\gamma-1}}
$$

The flow angle $\delta$ at $r=R_{s}$ is

$$
\begin{aligned}
\delta & =\arctan \left(\frac{1}{\gamma+1} \frac{R_{s}}{x}\right) \\
& =\arctan \left[\frac{\eta_{0}}{\gamma+1}\left(\frac{\pi C_{D}}{8}\right)^{1 / 4}\left(\frac{x}{d_{1}}\right)^{-1 / 2}\right]
\end{aligned}
$$


We now consider the equivalent conditions behind an oblique shock. The shock angle $\beta$ is assumed to be known - here we will use the fitted profiles from above. The Mach number and flow angle behind this shock, denoted by hatted variables to distinguish them from the equivalent blast wave quantities, are

$$
\begin{aligned}
\hat{M}_{1}^{2} & =\csc ^{2}(\beta-\hat{\delta}) \frac{2+(\gamma-1) M_{\infty}^{2} \sin ^{2} \beta}{2 \gamma M_{\infty}^{2} \sin ^{2} \beta-(\gamma-1)} \\
\tan \hat{\delta} & =2 \cot \beta \frac{M_{\infty}^{2} \sin ^{2} \beta-1}{M_{\infty}^{2}(\gamma+\cos 2 \beta)+2} .
\end{aligned}
$$

The pressure ratio through the oblique shock is

$$
\frac{\hat{p}_{1}}{p_{\infty}}=1+\frac{2 \gamma}{\gamma+1}\left(M_{\infty}^{2} \sin ^{2} \beta-1\right)
$$

The relations 6.2, 6.4, and 6.5 still hold here (with hatted variables replacing their non-hatted counterparts), and since for a perfect gas $\rho_{\infty} V^{2} / 2=\gamma p_{\infty} M_{\infty}^{2} / 2$, we have

$$
\hat{p}_{\text {stag }}^{\prime}=\frac{2}{\gamma M_{\infty}^{2}} \frac{\hat{p}_{\text {stag }}}{p_{\infty}} .
$$

In Figure 6.2, the stagnation pressure coefficients $p_{\text {stag }}^{\prime}$ and $\hat{p}_{\text {stag }}^{\prime}$ and flow angles $\delta$ and $\hat{\delta}$ are compared. The flow angles all agree reasonably well, with the $M=50$ and $M=100$ profiles lying slightly closer to the blast wave curve than the $M=20$ profile does. As for the stagnation pressure coefficient, the blast wave solution approximates the $M=20$ profile quite poorly, whereas the $M=100$ profile is approximated reasonably well over the domain considered. Agreement with the $M=50$ pressure coefficient profile is reasonable for $x / d_{1} \lesssim 25$, but diverges considerably after this. At this Mach number, the force coefficients predicted by the blast wave methodology showed good agreement with computational results for $x / d_{1} \approx 2,4.5$. Given that the oblique value drops by approximately $20 \%$ from $x / d_{1}=5$ to $x / d_{1}=25$, whereas the blast wave value drops by only a small amount, this gives a rough limit for the 
distance downstream to which we may expect the theory to give reasonable results. For higher Mach numbers, of course, this limit will be somewhat greater; for lower Mach numbers, somewhat smaller.
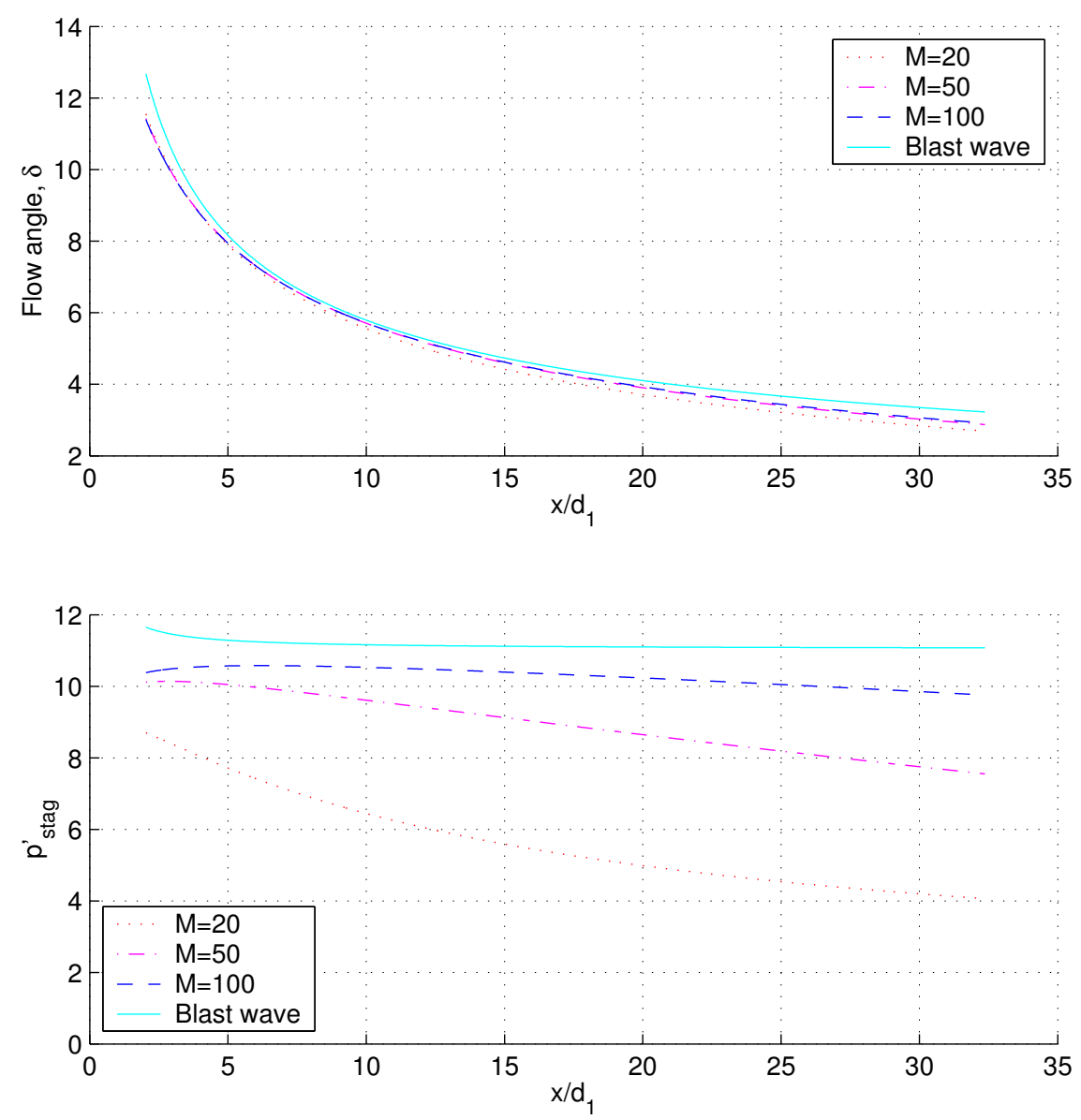

Figure 6.2: Flow angle (top) and stagnation pressure coefficient $p_{\text {stag }}^{\prime}$ (bottom) immediately behind blast wave and computed oblique shocks.

For asteroids entering a planetary atmosphere, the minimum entry velocity is the escape velocity for that planet $(11.2 \mathrm{~km} / \mathrm{s}$ for Earth, $10.4 \mathrm{~km} / \mathrm{s}$ for Venus) but the actual entry velocity will typically be significantly higher than this value. For Earth, the minimum entry velocity corresponds to a minimum Mach number in the upper atmosphere of $M \approx 35$, but a value of 50 or higher is more likely. Within the constraints of the perfect gas assumption, then, we may feel reasonably confident in using the blast wave methodology to model asteroid entry for separation distances of 
up to $\sim 20 d_{1}$. For higher entry velocities, we may extend this even further.

Finally, note that the blast wave methodology will not be valid for small values of $x / d_{1}$ (i.e., for $x / d_{1} \lesssim 1.5$ ). Although this part of the solution is not shown in Figure 6.2 , the singularity at $x=0$ in the blast wave solution is not present in the computed flows and leads to a considerable discrepancy, particularly in the value of $p_{\text {stag }}^{\prime}$, in this region of the flow.

\subsection{Extension of Blast Wave Methodology}

We now wish to extend the methodology developed in Chapter 2 to enable us to model, in particular, the forces on the secondary body when the primary bow shock is impinging upon it. We shall do this using a combination of the blast wave analogy and the oblique shock relations. As we saw in the previous section, the blast wave methodology becomes increasingly questionable as the downstream displacement is increased - it is hoped that by including the oblique relations, the domain of validity may be extended beyond what it would otherwise be.

\subsubsection{Lift Coefficients}

Consider the computational lift profiles seen in Figures 4.7 to 4.10 . The lift typically reaches a minimum value at the point where the primary shock first begins to impinge on the secondary body, which we assume to occur when the center of the sphere is at $r=R_{s}-d_{2} / 2$, i.e., when the outside edge of the sphere is level with the shock. As the lateral displacement is increased past this point, the lift coefficient increases in a roughly linear fashion to a maximum value at $r \approx R_{s}$ and then falls away again. As the flow configuration at the point $r=R_{s}$ lends itself somewhat readily to simple approximations, in that which follows we shall attempt to calculate the lift and drag coefficients in this situation. 
At the point $r=R_{s}$, we may crudely model the top half of the body as being exposed to singly-shocked flow and the lower half as being exposed to doubly-shocked flow, all rotated by an angle $\delta$ corresponding to the flow angle immediately behind the oblique shock. The geometry of this situation is shown in Figure 6.3. Note that the use of the oblique shock flow angle rather than the corresponding blast wave angle will introduce the freestream Mach number into the solution, whereas previously the methodology was independent of Mach number (or more correctly, assumed an infinite freestream Mach number). To determine the flow angle behind the oblique shock, we require knowledge of the oblique shock angle. In the previous section we used a fitted profile to provide this angle, but given the close agreement that was observed at high Mach numbers between the shock angles of these fitted profiles and the blast wave shock, we shall henceforth assume the shock angle to be given by the blast wave analogy profile.

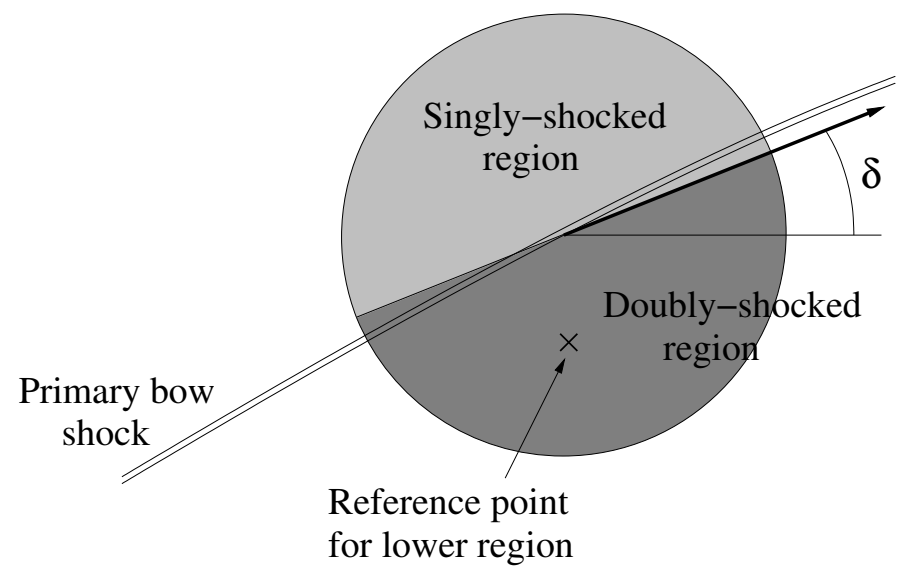

Figure 6.3: Approximation of flow configuration for calculation of the drag and lift coefficients at $r=R_{s}$, corresponding to the assumed maximum $C_{L}$ position.

To calculate the force coefficients in this configuration, we assume that the pressure distribution on the surface of the sphere is still given by the modified Newtonian distribution, but with differing pressure coefficients on top and bottom. On the 
upper, singly-shocked side, $p_{\text {stag }}^{\prime}$ is given by

$$
p_{\text {stag }}^{\prime}=\frac{2}{\gamma M_{\infty}^{2}}\left(1+\frac{2 \gamma}{\gamma+1}\left(M_{\infty}^{2}-1\right)\right)\left(1+\frac{\gamma-1}{2} M_{2}^{2}\right)^{\frac{\gamma}{\gamma-1}}
$$

where

$$
M_{2}^{2}=\frac{(\gamma-1) M_{\infty}^{2}+2}{2 \gamma M_{\infty}^{2}-(\gamma-1)}
$$

We have also used $p_{\infty}^{\prime}=\frac{2}{\gamma M_{\infty}^{2}}$. The contribution to the lift coefficient from this upper side is

$$
\begin{aligned}
C_{L}^{u}= & -\frac{1}{\pi} \int_{0}^{\pi} \int_{0}^{\pi / 2}\left(p_{\text {stag }}^{\prime}-p_{\infty}^{\prime}\right) \cos ^{2} \theta \hat{y} \sin \theta d \theta d \phi \\
& -\frac{1}{\pi} \int_{0}^{\pi} \int_{0}^{\pi} p_{\infty}^{\prime} \hat{y} \sin \theta d \theta d \phi \\
= & \frac{p_{\text {stag }}^{\prime}-p_{\infty}^{\prime}}{8}(2 \sin \delta-\cos \delta)-p_{\infty}^{\prime} \cos \delta,
\end{aligned}
$$

where $\hat{y}=\cos \delta \sin \phi \sin \theta-\sin \delta \cos \theta$.

Similarly, the contribution to the drag coefficient from the upper side is

$$
C_{D}^{u}=\frac{p_{\text {stag }}^{\prime}-p_{\infty}^{\prime}}{8}(2 \cos \delta+\sin \delta)-p_{\infty}^{\prime} \sin \delta
$$

On the lower side, the relevant pressure coefficients are the blast wave values $p_{1}^{\prime}$ and $p_{\text {stag }}^{\prime}$, as in Section 2.4. Note that $p_{\text {stag }}^{\prime}$ differs here from 6.12 in that the conditions before the normal shock are the blast wave conditions rather than the freestream conditions. In Chapter 2 we took the sphere's center as our reference point for the calculation of these pressure coefficients, but in the configuration being considered here, this point will no longer be suitable. A reasonable choice seems to be the point at the same distance downstream but midway between the center and the inside edge of the sphere, i.e., if we denote this point by $r_{r e f}$, then $r_{r e f}=R_{s}-d_{2} / 4$. This choice is shown in Figure 6.3. The contribution to the lift coefficient from the lower side, 
given the current assumptions, will be

$$
C_{L}^{l}=\frac{p_{\text {stag }}^{\prime}-p_{1}^{\prime}}{8}(2 \sin \delta+\cos \delta)+p_{1}^{\prime} \cos \delta
$$

For the contribution to the drag coefficient, however, we wish to include the derivatives of $p_{s t a g}^{\prime}$ and $p_{1}^{\prime}$ with respect to $x$, as we saw in Section 2.4 that the inclusion of these terms generally resulted in improved agreement with the computational profiles. With these terms, the contribution to the drag coefficient from the lower half of the sphere may be written

$$
\begin{aligned}
C_{D}^{l}= & \frac{p_{\text {stag }}^{\prime}-p_{1}^{\prime}}{8}(2 \cos \delta-\sin \delta)-p_{1}^{\prime} \sin \delta \\
& -\frac{1}{30} \frac{d_{2}}{d_{1}} \frac{\partial\left(p_{\text {stag }}^{\prime}-p_{1}^{\prime}\right)}{\partial\left(x / d_{1}\right)}\left(2 \cos ^{2} \delta+1-\frac{8}{\pi} \sin \delta \cos \delta\right)-\frac{1}{3} \frac{d_{2}}{d_{1}} \frac{\partial p_{1}^{\prime}}{\partial\left(x / d_{1}\right)} .
\end{aligned}
$$

However, having noted in the previous section that the value of $p_{\text {stag }}^{\prime}$ in the blast wave solution near the shock can diverge downstream from that for the oblique shock, we multiply $C_{L}^{l}$ and $C_{D}^{l}$ by the ratio of the oblique shock value to the blast wave value at $r=R_{s}$, which is $\hat{p}_{\text {stag }}^{\prime} / p_{\text {stag }}^{\prime}$ in the notation of the previous section. The resulting $C_{L}$ value, which we assume to be the maximum value in the profile, is

$$
C_{L}^{\max }=C_{L}^{u}+\frac{\hat{p}_{\text {stag }}^{\prime}}{p_{\text {stag }}^{\prime}} C_{L}^{l}
$$

and the corresponding $C_{D}$ value:

$$
C_{D}=C_{D}^{u}+\frac{\hat{p}_{\text {stag }}^{\prime}}{p_{\text {stag }}^{\prime}} C_{D}^{l}
$$

The resulting profiles as functions of the diameter ratio $d_{1} / d_{2}$ are plotted in Figure 6.4 for a freestream Mach number of 50 and $x / d_{1} \approx 2.07$ (this value corresponds 
to a center-to-center downstream displacement of 3 primary body radii). Also plotted are values obtained from an AMROC simulation with the same parameters. The technical details of this computation are identical to the $M=50$, diameter ratio 4 computations in Chapter 3. Agreement of the $C_{L}$ profile with the computational values is remarkably good, considering the crudity of the approximations that have been made. The $C_{D}$ profile agrees well for smaller values of $d_{1} / d_{2}$, but less well for larger values. Note, however, that there was some uncertainty involved with the location of the shock in the computations, and any resulting error in the positioning of the spheres will affect those of a smaller diameter most greatly.
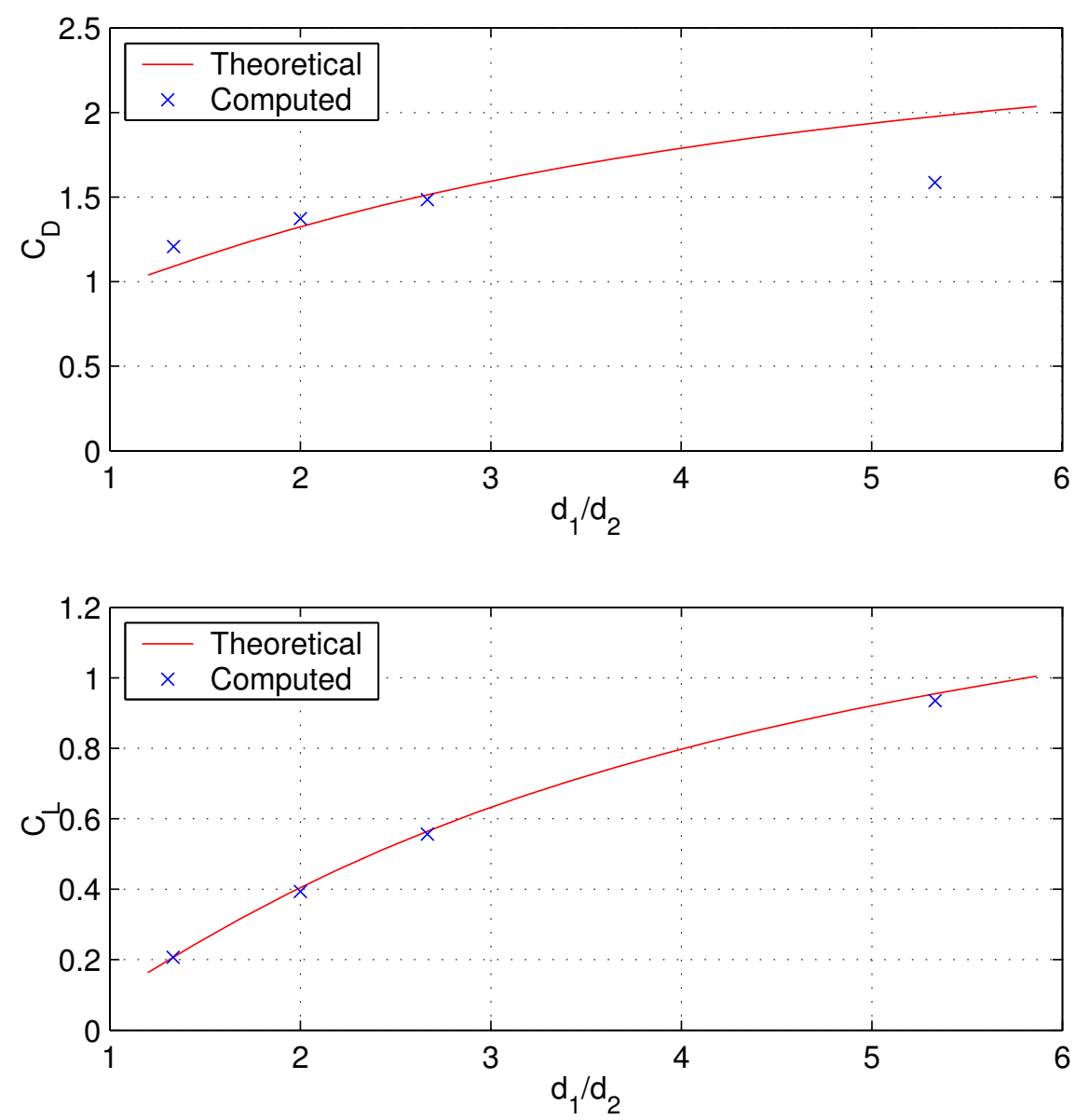

Figure 6.4: Computed and theoretical force coefficients at $r=R_{s}$ as a function of diameter ratio for a downstream displacement of 3 primary body radii and Mach number of 50 . 
So, our semi-empirical description of the lift coefficient profile is as follows. For $r<R_{s}-d_{2} / 2$, the lift coefficient is given by the blast wave methodology of Section 2.4, using the modified Newtonian pressure distribution and including $x$-derivatives. If $C_{L}^{\min }$ is the value given by the methodology at $r=R_{s}-d_{2} / 2$, the value for $C_{L}$ in the range $r \in\left(R_{s}-d_{2} / 2, R_{s}\right)$ is obtained by a linear interpolation between $C_{L}^{\min }$ and the value $C_{L}^{\max }$ from 6.18. As $r$ is increased further, the lift coefficient must eventually revert to its freestream value of zero; this is assumed to occur at $r=R_{s}+d_{2} / 2$. Between $r=R_{s}$ and $r=R_{s}+d_{2} / 2$, we assume that the drop in lift also occurs linearly.

\subsubsection{Drag Coefficients}

We now turn to the drag coefficient profiles. These are generally less easily characterised than the lift profiles, so we must be a little careful. Using the description above, we have already obtained reasonable values when the center of the sphere is at $r=R_{s}$, so we shall seek to extend this description for $r<R_{s}$. We assumed at $r=R_{s}$ that the sphere was cut by a plane through its center into a singly-shocked region above and a doubly-shocked region below. For $r<R_{s}$, we also assume that the sphere is cut by a plane into these two regions, with the plane passing through $R_{s}$ and rotated by the flow angle $\delta$. However, when the center of the sphere is at $r=R_{s}$, we have used the value of $\delta$ given by the oblique shock relations, whereas at $r=R_{s}-d_{2} / 2$ we use the blast wave value, so for points in between we use a linear bridging function between the oblique shock and blast wave values. The geometry in this situation is shown in Figure 6.5.

We again use the modified Newtonian pressure distribution on the surface of the sphere, with differing pressure coefficients in the two regions. If $\alpha$ is the angle that separates the singly- and doubly-shocked regions, as shown in Figure 6.5, it may be 


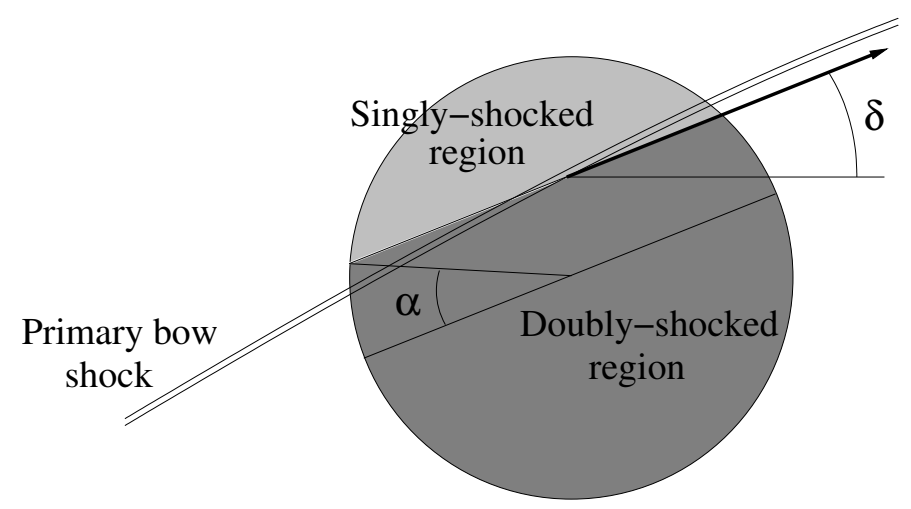

Figure 6.5: Approximation to the flow geometry used to calculate the drag coefficient for shock impingement with $r<R_{s}$.

shown that the contribution to the drag coefficient from the upper region is given by

$$
\begin{aligned}
C_{D}^{u}= & \left(p_{\text {stag }}^{\prime}-p_{\infty}^{\prime}\right)\left[\frac{1}{8} \sin \delta \cos ^{4} \alpha+\frac{\cos \delta}{4 \pi}\left(\pi-2 \alpha-\frac{4}{3} \sin 2 \alpha-\frac{1}{6} \sin 4 \alpha\right)\right] \\
& +p_{\infty}^{\prime} \sin \delta \cos ^{2} \alpha
\end{aligned}
$$

On the lower region we use the blast wave analogy as at $r=R_{S}$, but it is not immediately clear at which reference point, $r_{r e f}$, we should take the blast wave variables. At $r=R_{s}-d_{2} / 2$, the reference point coincides with the center of the sphere (i.e., $\left.r_{r e f}=r\right)$, whereas at $r=R_{s}$, our choice of reference point is $r_{r e f}=R_{s}-d_{2} / 4$. We could use a simple linear bridging function between these two values, but this does not make much physical sense, for the following reason. For $r$ only slightly greater than $R_{s}-d_{2} / 2$, the fact that the shock is impinging near the top of the sphere will make little difference to the conditions near the front of the sphere that contribute most to the drag. Thus, for $r$ close to $R_{s}-d_{2} / 2$, we would like $r_{r e f} \sim r$. The simplest function that satisfies this and the two boundary conditions is the quadratic function $r_{r e f}=r-\left(R_{s}-d_{2} / 2-r\right)^{2} / d_{2}$.

We may then obtain the contribution to the drag coefficient from the lower region of the sphere. The full expression is rather lengthy and is given in Appendix C, along with the full derivation of the drag coefficient in the impinging situtation. 
This expression simplifies to Equation 6.17 for $r=R_{s}$. As we multiplied this latter expression by $\hat{p}_{\text {stag }}^{\prime} / p_{\text {stag }}^{\prime}$ to obtain the full drag coefficient, to ensure continuity at $r=R_{s}$ we multiply the lower contribution in the impinging case by a function that varies linearly between 1 at $r=R_{s}-d_{2} / 2$ and $\hat{p}_{\text {stag }}^{\prime} / p_{\text {stag }}^{\prime}$ at $r=R_{s}$.

Thus we have our expression for the drag coefficient in the case $r \in\left(R_{s}-d_{2} / 2, R_{s}\right]$. For $r>R_{s}$, this coefficient, like the lift coefficient, is assumed to drop off linearly to the freestream value (in this case 0.88 ) at $r=R_{s}+d_{2} / 2$. For $r<R_{s}-d_{2} / 2$, we again use the methodology from Section 2.4 with the modified Newtonian distribution and including $x$-derivatives. We will, however, drop the $r$-derivatives from the drag expression, as these contribute negligibly to the drag but would cause a slight discontinuity at $r=R_{s}-d_{2} / 2$.

Finally, we observed in Chapter 4 that the theoretical drag coefficient profile drops off with decreasing $r / R_{s}$ more rapidly than in the numerical simulations. In all the simulations, however, as $r \rightarrow 0$, the drag coefficient tends to a value of between 0.15 and 0.2 that is relatively independent of body size or downstream displacement. We thus set a minimum value for the drag coefficient of 0.15 in the shocked region.

The full drag and lift coefficient profiles that result from these adjustments are shown in Figure 6.6. The Mach number for the theoretical profiles is $M=50$, while both the $M=10$ and $M=50$ computations are shown, as there are not always sufficient points for $M=50$ alone to be able to make a good comparison. We have made no attempt to model the wake area, but forces are generally small there and so will not differ significantly from those provided by our semi-empirical description. Agreement between theoretical and computational profiles is generally quite good. Note that the minimum lift point occurs at a slightly smaller value of $r / R_{s}$ in the theoretical profiles than in the computational profiles. We may attribute this to the fact that the shock radius is smaller in the theoretical case, so $R_{s}-d_{2} / 2$, the value of $r$ at which the minimum lift is assumed to occur, also takes a smaller value. 

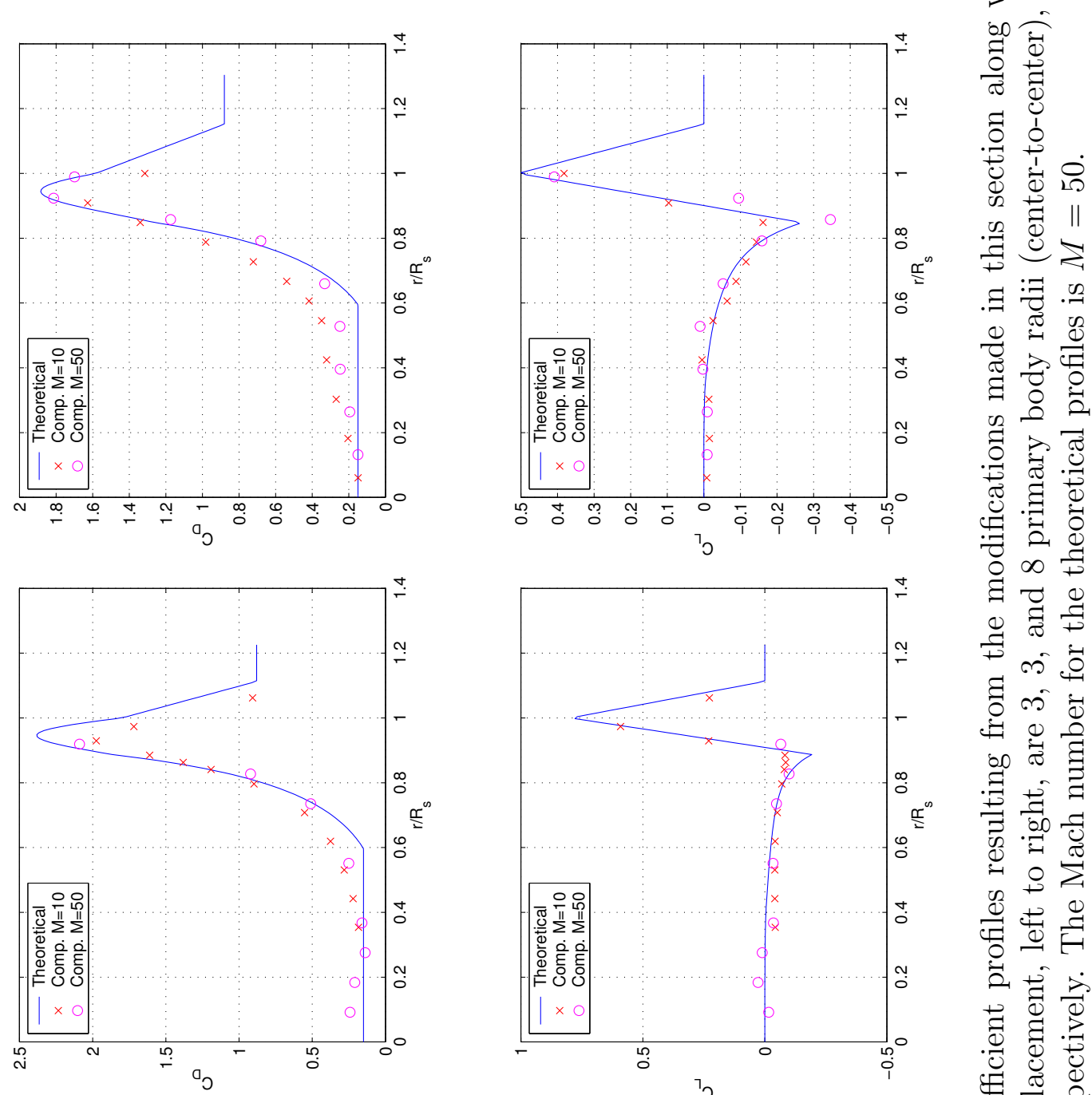

밍

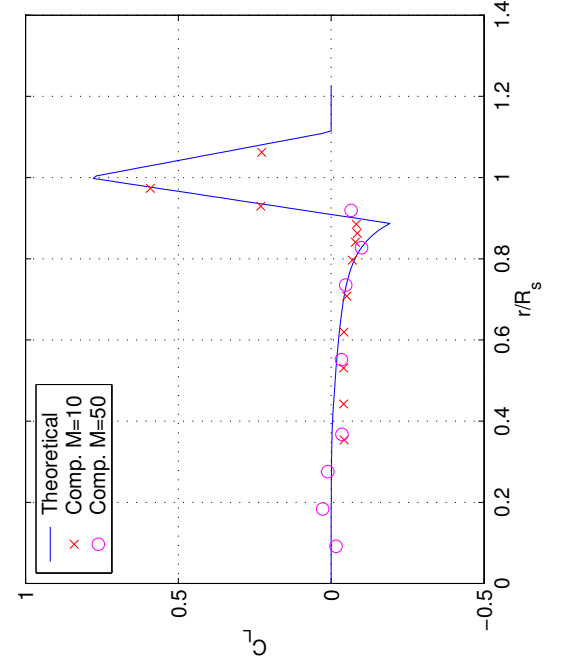

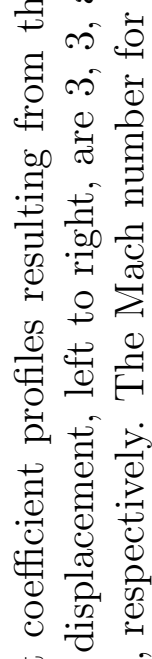
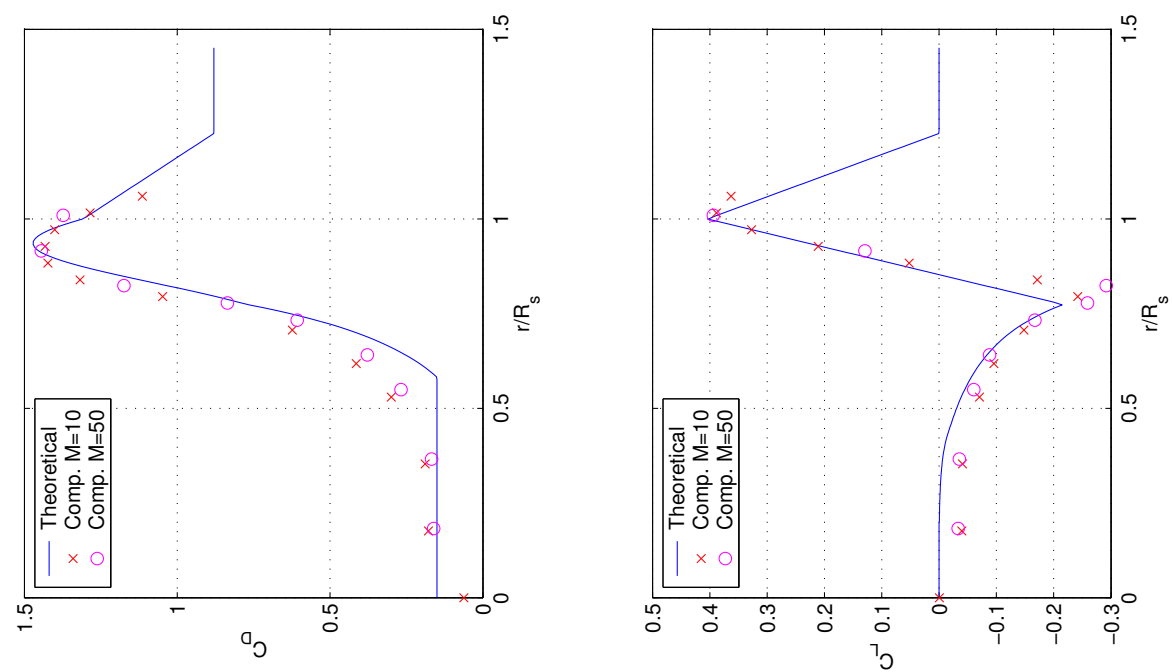

急灵

चี

कo

ส

चี ஏ

串

तु

สี

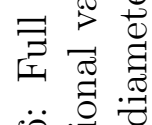

$\ddot{0}$

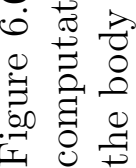




\subsection{Simulating the Planetary Entry of a Binary Asteroid System}

\subsubsection{Simulations of the Asteroid 2000 DP107}

We now focus our attention on the near-Earth asteroid 2000 DP107. Radar images obtained by Margot et al. (2002) have shown this asteroid to be composed of two bodies: an $~ 800$ meter diameter primary and an $~ 300$ meter diameter secondary orbiting around their common center of mass. The orbital semimajor axis is $2620 \pm 160$ meters and the orbital period is $1.755 \pm 0.007$ days, which constrain the total mass of the system to $4.6 \pm 0.5 \times 10^{11}$ kilograms and the bulk density of the primary to $1.7 \pm 1.1 \times 10^{3}$ kilograms per cubic meter. The primary body is roughly spherical and has a rotation period of $2.7755 \pm 0.0002$ hours, which is near the breakup limit for a strengthless body of this size. Such rotation rates are typical of primary bodies in other observed binary systems, suggesting these bodies to be gravitationally-bound aggregrates rather than monoliths.

The heliocentric orbit of asteroid 2000 DP107 has a semimajor axis of 1.37 AU, an eccentricity of 0.38 , and an inclination of $8.7^{\circ}$, which brought it within $0.048 \mathrm{AU}$ of Earth on 19 September 2000. In this section, we will model a somewhat closer approach in which this binary system enters the Earth's atmosphere. In particular, we will investigate the effect of aerodynamic interactions on the relative displacement of the two bodies at the point of impact. We will also seek to determine the effect that these interactions have on the likelihood that a single impact crater is formed rather than a doublet crater. 


\subsubsection{Simulation Parameters}

We begin by assuming that the orbital parameters of the binary system at the point of atmospheric entry have been unperturbed by the approach; in particular, we assume that tidal forces do not disturb the orbital configuration (more shall be said about this shortly). For the 2000 DP107 system, the task of simulating an atmospheric entry is made easier by the fact that the value of the eccentricity of the secondary's orbit is only 0.01 , which means we may to a very good approximation treat the orbit as circular. Even with this simplification, however, there are many variables that must still be considered.

We assume that the initial entry velocity of the primary body lies in the $x-z$ plane, where the $z$ axis is normal to the surface of the Earth (which we assume to be flat). We denote the angle made by the velocity vector with the $x$-axis by $\theta$ and the magnitude of the velocity by $V$. The angle $\theta$ may take any value from 0 to $\pi / 2$, with $\pi / 4$ being the most likely entry angle. The minimum value for $V$ is $11.2 \mathrm{~km} / \mathrm{s}$, corresponding to the Earth's escape velocity, with values of up to around $70 \mathrm{~km} / \mathrm{s}$ possible. We will need two further angles to describe the orientation of the plane of the secondary's orbit relative to the $x$ - $z$ plane, and another angle to describe the position of the secondary within this orbit. This gives us five variables, just for this particular binary system, which is too large a parameter space to explore. Fortunately, we can make further simplifications.

First, for the given orbital period and semimajor axis, the orbital speed is of the order of $0.1 \mathrm{~m} / \mathrm{s}$, and this will be typical of such systems, given the weakness of the gravitational attraction between bodies of such sizes. This initial orbital velocity is negligible, given that the atmosphere will be traversed within a few seconds, and so we may treat the secondary as initially stationary relative to the primary. As the initial relative position of the secondary is limited to points described by the surface of a sphere with the primary at the center, we may replace the three angles describing 
the secondary's orbit by two angles describing the secondary's initial position relative to the primary. These angles, which we denote by $\phi$ and $\psi$, are shown in Figure 6.7. The figure should be interpreted as follows. If we initially place the secondary body at the point $(a, 0,0)$, we first rotate by an angle $\theta$ about the $y$-axis - this also gives the rotated $x^{\prime}$ - and $z^{\prime}$-axes. Subsequently, a rotation of $\psi$ about the $z^{\prime}$-axis and a further rotation of $\phi$ about the $y$-axis are performed.

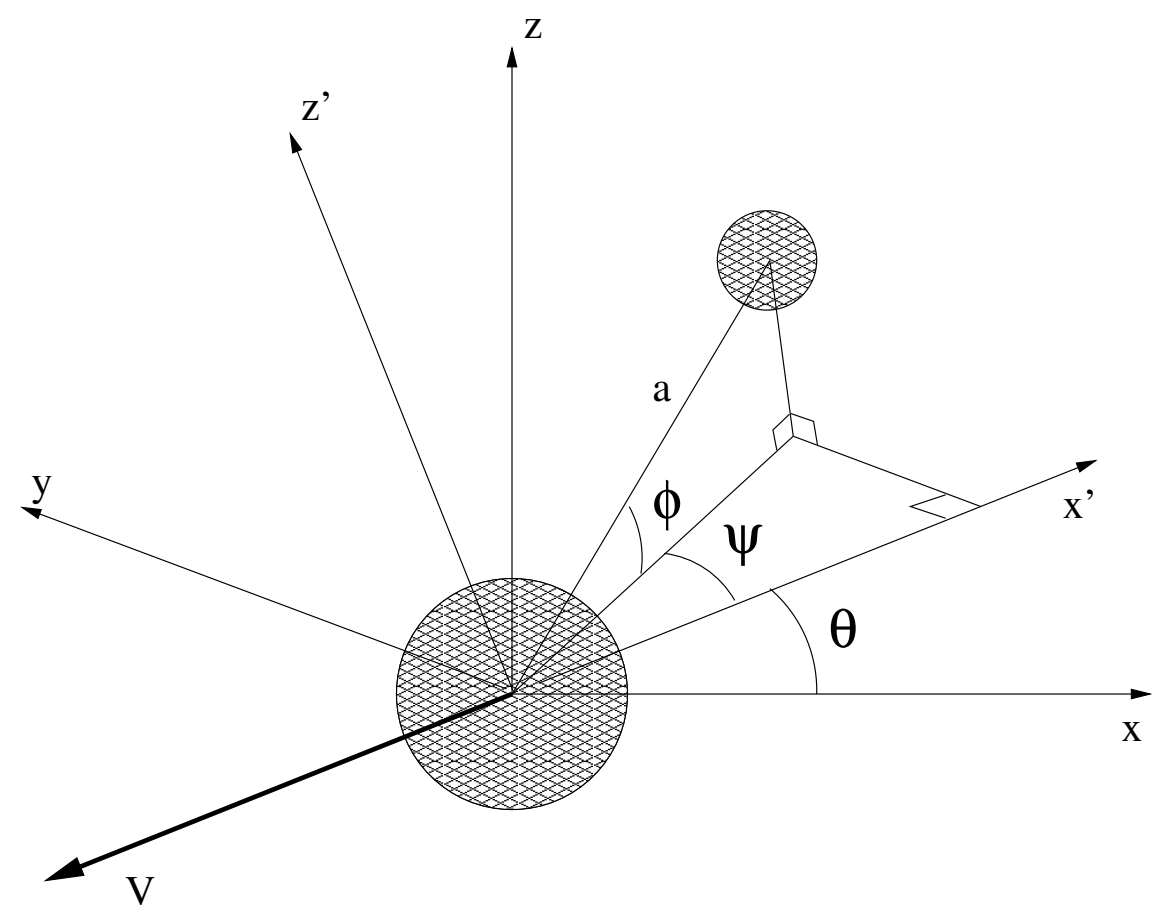

Figure 6.7: Initial binary system configuration for atmospheric entry

We now consider the entry velocity $V$. For bodies of the size of those in the 2000 DP107 system, this velocity will change by only a small amount during the flight time, and the relative displacement of the two bodies will typically be small compared to the shock radius (although in the impinging case, the secondary radius is the more relevant length). The forces on the secondary body are given by $\frac{1}{2} \rho(z) C_{i} V^{2} A$, where $C_{i}$ is the force coefficient in the $i$-th direction. In the blast wave methodology, however, we assume $C_{i}$ to depend only on the position of the secondary relative to the primary (and to a small degree the Mach number, but this will also change only 
very little during the flight). We also assume that the mass and area are constant throughout the flight - more will be said about these assumptions shortly. The total displacement of the secondary relative to the primary in the $i$-th direction due to aerodynamic loading will then be

$$
\begin{aligned}
s_{i} & =\int_{0}^{t_{0}} \int_{0}^{t} a_{i}(\tau) d \tau d t \\
& =\int_{0}^{t_{0}} \int_{0}^{t} \frac{1}{2} \rho(\zeta(\tau)) C_{i} V^{2} \frac{A}{m} d \tau d t \\
& =\int_{z_{0}}^{0} \int_{z_{0}}^{z} \frac{1}{2} \rho(\zeta) C_{i} V^{2} \frac{A}{m} \frac{d \tau}{d \zeta} \frac{d t}{d z} d \zeta d z \\
& \approx \frac{1}{2} C_{i} \frac{A}{m \sin ^{2} \theta} \int_{z_{0}}^{0} \int_{z_{0}}^{z} \rho(\zeta) d \zeta d z,
\end{aligned}
$$

which is independent of the entry velocity $\left(z_{0}\right.$ is the height at which the atmosphere is assumed to begin).

We are thus left with three parameters: the entry angle $\theta$ and the two angles describing the position of the secondary relative to the primary, $\phi$ and $\psi$. For a given value of $\theta$, then, we vary $\phi$ and $\psi$ over the range of values in which shock wave interactions will be important. For each combination of values, the trajectories of the two bodies are integrated from a height of $95 \mathrm{~km}$. Atmospheric information is taken from the Smithsonian reference tables (Forsythe, 1954), with linear interpolation between values. The primary body is assumed to generate a shock shape as given by the blast wave analogy, with the shock stand-off distance assumed to be zero. The aerodynamic force acting on the primary body is simply $\frac{1}{2} \rho(z) C_{D} V^{2} A$ in the direction of travel, where $C_{D}$ is assumed to take the value 0.88 . The aerodynamic forces on the secondary are given by the extended blast wave methodology if part of the secondary is inside the primary shock; otherwise, the force is simply the drag force in the direction of travel. Acceleration due to gravity is included, though it will have a negligible effect on bodies of these sizes. The integration is continued until both bodies have reached 
ground level.

At the point of impact, the bodies are assumed to create craters with sizes given by an energy-diameter scaling law. The law that shall be assumed here was proposed by Gault (1974) and used by Passey and Melosh (1980) to describe impacts producing craters of kilometer dimensions:

$$
D=0.027 \rho_{m}^{1 / 6} \rho_{t}^{-1 / 2} E_{k}^{0.28} \sin ^{1 / 3} \theta_{f}
$$

where $D$ is the crater diameter, $\rho_{m}$ and $\rho_{t}$ are the densities of the meteoroid and target respectively, $E_{k}$ is the kinetic energy of the relevant body and $\theta_{f}$ is the impact angle relative to the horizontal. All variables are in cgs units. For these simulations, we

will assume a target density for Earth of $3 \mathrm{~g} / \mathrm{cm}^{3}$. It is not clear exactly the amount of separation needed between the two craters in order that they may be recognized as a doublet rather than a single crater; we shall use the criterion that if the center of the secondary crater lies outside the primary crater, a doublet crater is recognizable.

\subsubsection{Model Assumptions}

The assumptions of the model during the atmospheric transit are as follows:

1. The rotation rates of the two bodies and the relative velocity are negligible.

2. Both bodies are spherical and remain so, with no mass loss throughout the flight time.

3. The atmosphere behaves as a perfect gas.

4. The curvature of the Earth is negligible.

We have already deduced that the initial relative velocities of the two bodies in such binary systems may be ignored. During the course of the atmospheric transit the bodies will experience different accelerations, but, for bodies the size of those in 
question, the resulting relative velocity will be small compared to the velocity of the system. To show this, for each of the simulations of 2000 DP107, the final velocities of the two bodies were recorded. For an entry angle of $\pi / 8$, the root-mean-square velocity difference over all simulations was around $1 \%$ of the final primary velocity, with the maximum difference being approximately $4 \%$ of this value. For an entry angle of $\pi / 16$, the mean and maximum differences were around $2 \%$ and $9 \%$, respectively. For a $9 \%$ difference this assumption becomes a little questionable, especially considering the $V^{2}$ dependence of the forces, but, given the other assumptions of the model, is not cause for too much concern.

As for rotations, the surface of the 2000 DP107 primary is initially moving at approximately $2.5 \mathrm{~m} / \mathrm{s}$, which is again negligible compared to the entry velocity. During the atmospheric transit, if the bodies are spheres of uniform composition, the moments generated will be negligible. Real bodies will of course deviate from perfect sphericity, but given the size of the bodies (and thus the extremely large rotational inertias), it is unlikely that rotations would become important during the flight time.

Assumption (2) is somewhat questionable, however. While radar images have indicated that the primary body is roughly spherical, it probably owes this shape to its strengthless composition. Thus, on entering the atmosphere it will be subject to deformation - indeed, authors have used hydrodynamic equations of state to describe such an entry. The loading will tend to flatten the primary body, leading to a broadening of the primary bow shock. Given the size of the primary body, however, and thus the length of time necessary for an induced pressure wave to travel throughout the body, we do not expect this deformation to be too significant. Also, the dependence of the shock radius on primary diameter is relatively weak - at a given point downstream, $R_{s} \sim \sqrt{d_{1}}$. Any deformations or other deviations from sphericity of the secondary would be more significant, however, given that our analysis is very much tied to this geometry. As the secondary is smaller, it is more likely to be monolithic 
in nature, but at the same time less likely to be initially spherical. From the point of view of the assumption of sphericity, then, this modeling is of a somewhat idealized situation.

The assumption of constant mass is also open to question, as ablation will occur during the atmospheric transit. This effect could easily be incorporated into the model through the introduction of an ablation equation such as that found in Passey and Melosh (1980), but given the further uncertainty associated with the relevant constants, such as the heat transfer coefficient, it was deemed preferable to neglect the effects of ablation in the current simulations. Also, for bodies of the sizes considered here, the fraction of mass lost through ablation will typically be quite small.

While we may accept Assumption (2) as something of an idealization in the case of Earth, for Venus it will become hopelessly unrealistic. Asteroids hundreds of meters in size typically undergo catastrophic fragmentation in the dense atmosphere of Venus, leaving a characteristic radar-dark pattern on the surface of the planet (Bottke and Melosh, 1996). Obviously, an assumption of no deformations will become quite untenable in this situation, and it is for this reason that we will limit ourselves to an Earth entry in the simulations of this chapter.

Assumption (3) also represents something of an idealization. We observed in Section 5.4.4 that the effect of dissociation on the pressure behind a shock wave in a diatomic gas is quite small, although at the speeds in question, further real-gas effects, such as ionization, will become significant. Flow chemistry is also known to have an effect on shock shape - the shock over a wedge in dissociating flow, for example, is curved inwards from the oblique frozen shock. Thus, we might expect the shock angle to be affected near the primary body, which will modify the shock radius further downstream. If anything, however, the effect of dissociation will be to shift the shock inwards, bringing it closer to the blast wave analogy profile. Finally, Assumption (4) will be reasonable provided the angle of entry is not too shallow. 
In addition to these aerodynamic assumptions, we have also made note of the assumption that the orbital parameters of the system are unchanged during the approach to Earth. In fact, the influence of Earth's gravity could significantly affect the orbital configuration. Note, for example, that the force on the secondary body due to the Earth's gravity becomes larger than that due to the primary's gravity at a distance of approximately $10^{7} \mathrm{~km}$. If the average approach speed is $10 \mathrm{~km} / \mathrm{s}$, this distance will take approximately 6.5 orbital periods to cover. During this time, the orbital configuration could be strongly perturbed by the accumulative effect of tidal forces caused by the slightly different positions of the two bodies in the Earth's gravitational field. Tidal forces will also cause significant deformations to the strengthless bodies. Such effects are, however, outside the scope of this work and will not be considered further.

\subsubsection{Results}

Figures 6.8 through 6.10 show the results of simulations for three entry angles: $\pi / 4$, $\pi / 8$, and $\pi / 16$. In the left plot of each figure, the magnitude of the relative displacement of the two bodies on impact are plotted as a function of the angles $\phi$ and $\psi$, with contours plotted underneath. The entry velocity in each of these cases is 20 $\mathrm{km} / \mathrm{s}$. In the right plot of each figure, the profiles are shown on the slice $\psi=0$ and include entry velocities of $11.2,20$, and $50 \mathrm{~km} / \mathrm{s}$ as well as the profile obtained if no aerodynamic interactions are included. The horizontal lines in these plots indicate the radius of the primary crater produced at the relevant entry velocity. Thus, if the relative displacement curve lies above this line, a doublet crater will be formed according to our criterion; otherwise, the secondary crater will be indistinguishable from the primary crater.

The form of the displacement profiles are generally as one might expect, given the nature of the lift coefficients experienced by the secondary body in the current 


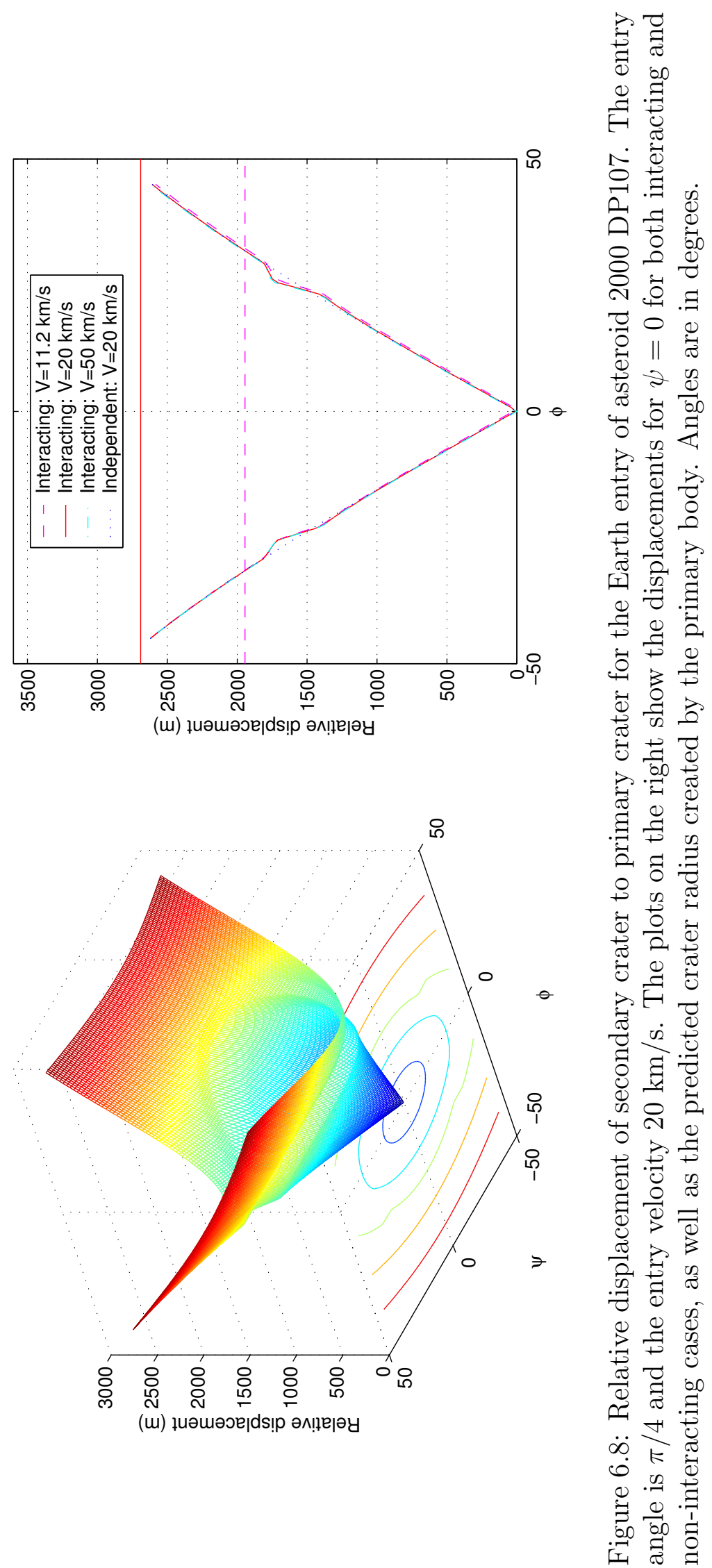




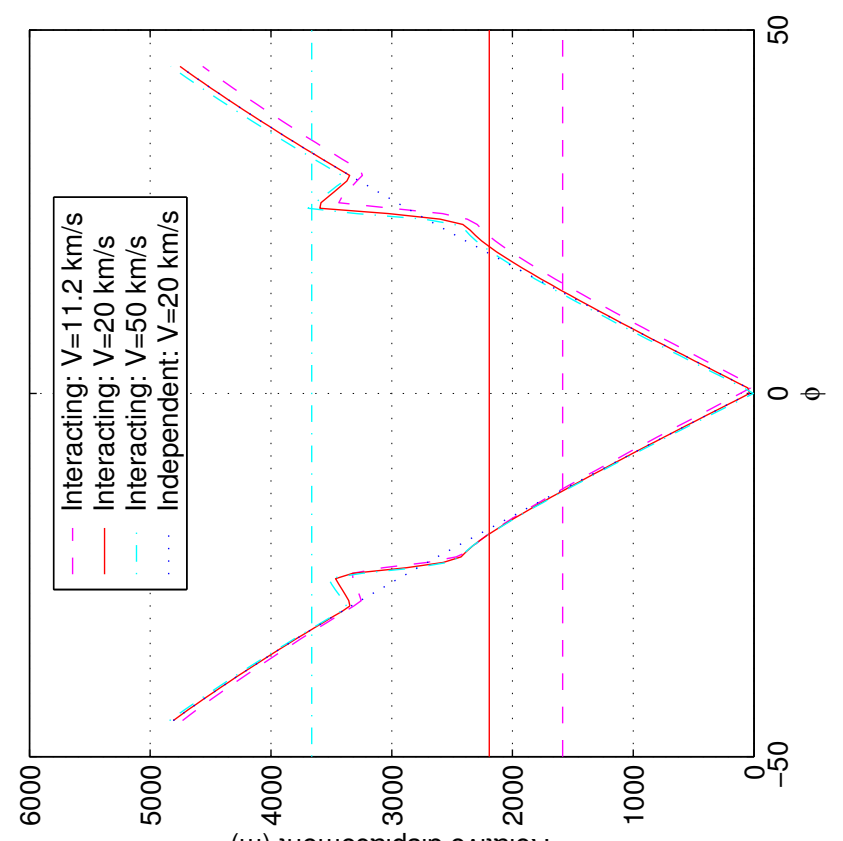

(u) ұиәшәэе|ds!̣ әм!ฺе|әу

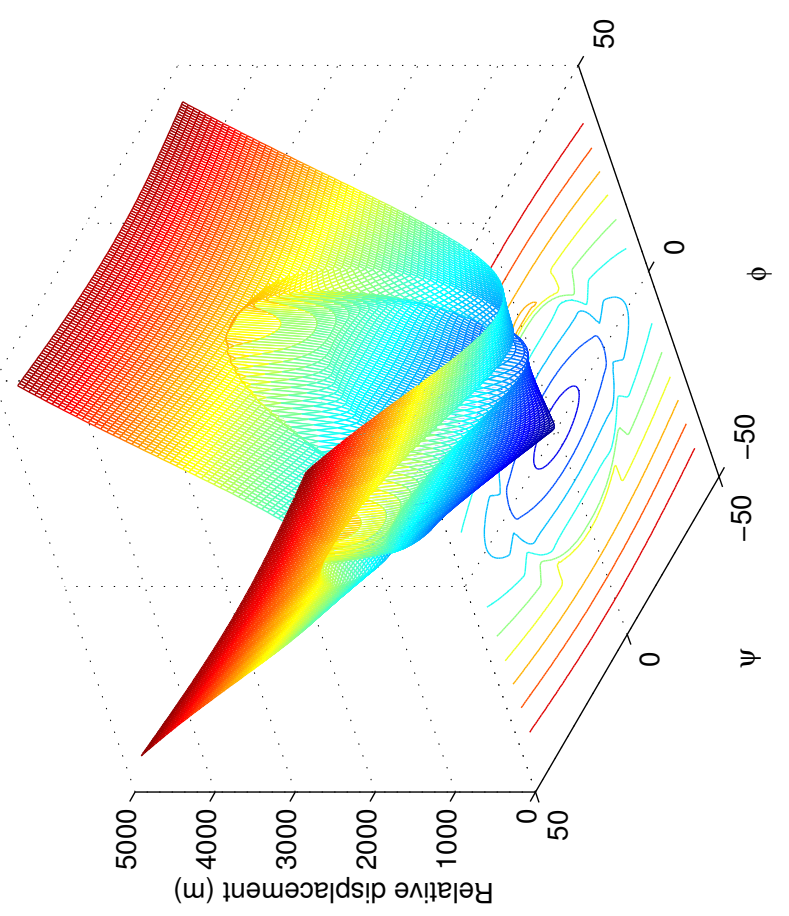

류뮤

幽

E

덩

커

คิ $\frac{1}{0}$

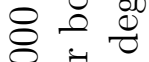

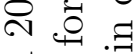

.0.0 0

की

ثै

贻

馬芯

건

तె

[I] $\widetilde{\Omega}$



$\stackrel{్}{\$} 0$

언

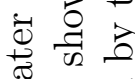

ठ러 엉

30

त्ञ

의 일

至

이 疋

苛

00

同

莺的总

仓 ్ㅓㅇ

फ

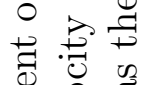

ฮี

ఫ्త

常

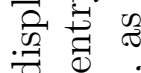

0 过

击

ब

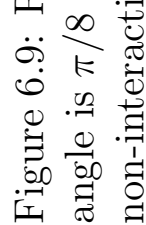




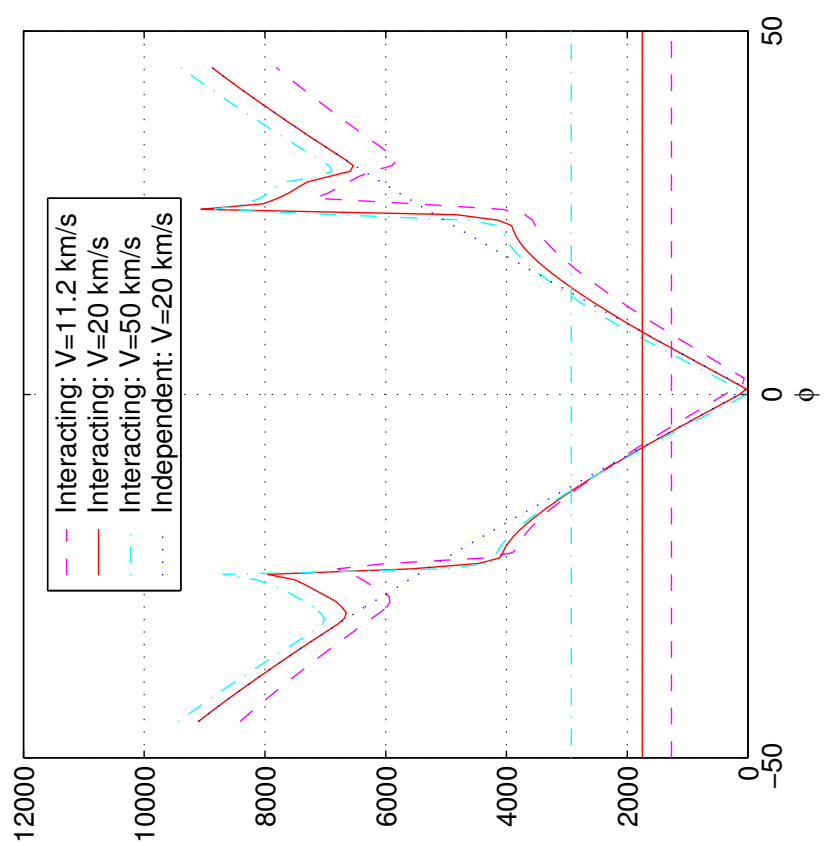

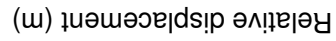

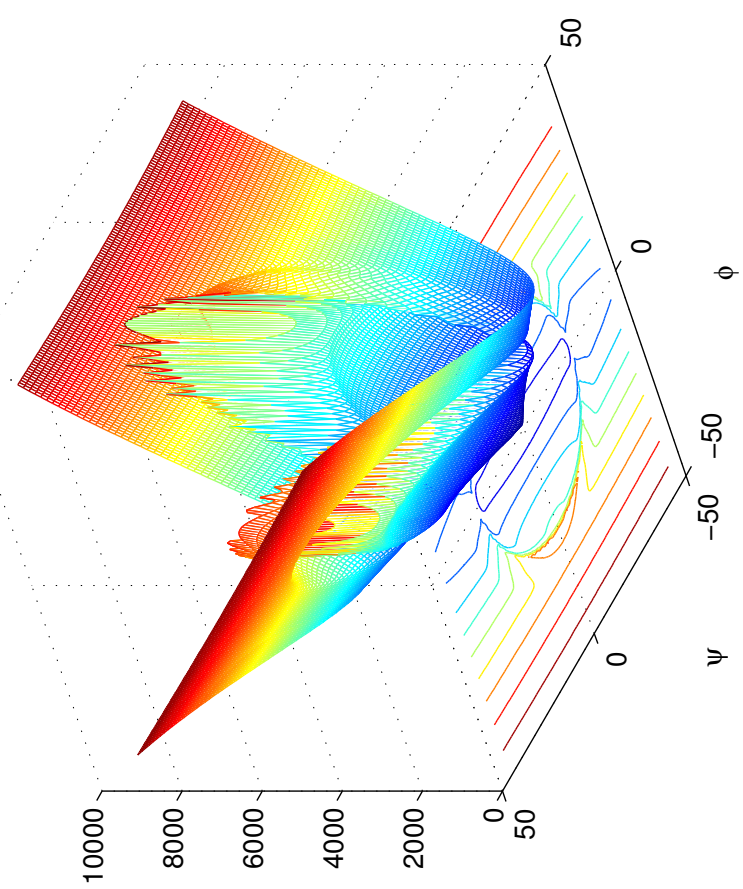

(u) łuәшәәе|ds!̣ әм!̣е|әу

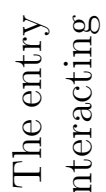

N

ค1 0

○.ี.

尺ำ

.

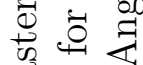

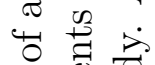

링

븡 仓

궁

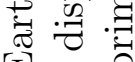

[I] 0 己

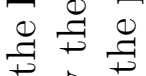

苛

उ) जू

$\underset{0}{0} \stackrel{0}{0}$

U ज

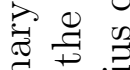

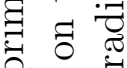

$\stackrel{\underline{0}}{+}$

ذِ

。

ए E ठ

完

్ㅣ일

舟

요 워

पै

류유

¿

过

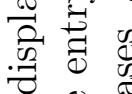

¿

离志

లీ

$\ddot{0} \stackrel{0}{=}$

당

0.

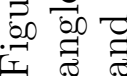


configuration. For small $\phi$ and $\psi$, the secondary body is initially positioned entirely within the primary shock, where entrainment will occur due to the attractive force experienced towards the primary axis of travel. The resulting relative displacement on impact will then be smaller than if no aerodynamic interactions were present. If the primary shock impinges in the initial configuration, however, the force will be repulsive, and this is observed in the sharp jump in relative displacement at certain values of $\phi$ and $\psi$. Note that, from the point of view of aerodynamic interactions, the relative displacement should be symmetric in $\phi$ and $\psi$. At the point of impact, however, this symmetry is lost.

The effect of aerodynamic interactions on the final relative displacement for $\theta=$ $\pi / 4$ is very small as the time available for interaction is relatively brief. As the entry angle becomes shallower the effect of the interactions becomes more noticeable. With bodies of these sizes, however, the entry angle needs to be quite shallow (of the order of $\pi / 16$ ) before the transit time is large enough that the aerodynamic effects are really significant. The effect of the entry velocity is quite small, as predicted, but also becomes more noticeable as the entry angle is decreased.

As the effect of aerodynamic interactions is relatively small for this asteroid system, the effect on the likelihood of a doublet rather than a single crater being formed will also be small. For $\theta=\pi / 4$, we see that there will in fact be no effect as, even at the slowest entry velocity, a doublet crater will be formed only if the bodies are initially configured such that no aerodynamic interactions will occur. For entry angles of $\pi / 8$ and $\pi / 16$, however, aerodynamic interactions will occur in the configurations for which the final relative displacement is near the transition point from a single to a doublet crater. Note that, away from $\psi=0$, these interactions will occur for configurations that result in smaller relative displacements than those at $\psi=0$. Thus, although the effect of interactions appears negligible in the $\psi=0$ slice of Figure 6.10 this will not necessarily be the case for other values of $\psi$. 
To quantify the effects of interactions on this likelihood, further simulations were run in which $\phi$ and $\psi$ were varied from $-\pi / 2$ to $\pi / 2$, corresponding to configurations in which the secondary is located on the downstream hemisphere of possible positions (and thus representing half of all possible configurations). These were carried out for both interacting and non-interacting cases and the numbers of simulations resulting in single rather than doublet craters were compared between the two. Differences were found to be negligible, however. For $\theta=\pi / 8$ and $V=20 \mathrm{~km} / \mathrm{s}$, for example, the fractions of impacts resulting in single craters were 0.1100 in the interacting case and 0.1103 in the non-interacting case, while for $\theta=\pi / 16$ and $V=50 \mathrm{~km} / \mathrm{s}$ the corresponding fractions were 0.109 and 0.108 respectively.

\subsubsection{Further Simulations}

As we have seen, for bodies of the size of those in the binary system 2000 DP107, aerodynamic effects during the atmospheric transit are relatively small. In this section we will simulate the planetary entry of a smaller system for which we would expect the effect of aerodynamic interactions to be more significant. As no binary systems have been observed with a primary diameter smaller than $\sim 200 \mathrm{~m}$, we will choose this as our primary body size. In addition to the entry angle, we will vary the size and orbital radius of the secondary to observe the effect that these parameters have on the final configuration.

In Figure 6.11 are plotted the final relative displacements for the entry of a system one quarter the scale of 2000 DP107 (i.e., secondary radius $37.5 \mathrm{~m}$, orbital radius 655.5 m), with an entry angle of $\theta=\pi / 4$. Comparing to Figure 6.8 , we see that the effect of aerodynamic interactions for these smaller bodies is far more significant. This may also be seen in Figure 6.12, in which this same system is simulated for $\theta=\pi / 8$.

In Figure 6.13 the secondary body radius is $37.5 \mathrm{~m}$, as in the previous two figures, but the initial orbital radius has been doubled to $1311 \mathrm{~m}$. The entry angle is $\pi / 4$. 
The final relative displacement profiles are similar to those in Figure 6.11, but the domain of aerodynamic influence is smaller at this radius as less solid angle is covered by the primary shock. In Figure 6.14 the orbital radius is $655.5 \mathrm{~m}$, but the secondary radius has been halved to $18.75 \mathrm{~m}$. The entry angle is again $\theta=\pi / 4$. In Chapter 2 we saw that the lift coefficient experienced by the secondary body became smaller (less negative) as the body size was decreased. Here we see that this results in relatively small aerodynamic effects in those cases in which the secondary remains entirely inside the primary shock during atmospheric transit. If the primary shock impinges on the secondary, however, large relative displacements still result.

As in the case of the larger system, for the most probable entry angle of $\theta=\pi / 4$ the effect of aerodynamic interactions on the likelihood of a doublet versus a single crater forming is very small. Only at the slowest entry speeds will the interactions produce any sort of effect. For shallower entry angles, however, such as in the configuration shown in Figure 6.12, we see that the interactions could substantially affect this likelihood. To quantify this, simulations were performed for the parameters considered in Figure 6.12 for $\phi$ and $\psi$ in the range $-\pi / 2$ to $\pi / 2$, and the numbers of simulations resulting in single rather than doublet craters were again compared between interacting and non-interacting cases. For an entry velocity of $20 \mathrm{~km} / \mathrm{s}$, the fraction of impacts resulting in a single crater was 0.179 for the interacting case and 0.203 for the non-interacting case. For an entry velocity of $50 \mathrm{~km} / \mathrm{s}$, the corresponding fractions were 0.406 and 0.437 , while for $11.2 \mathrm{~km} / \mathrm{s}$ they were 0.080 and 0.088 respectively. We see then that in all cases the interactions increase the overall probability that a doublet crater will be formed, albeit by a relatively small amount. This increase is a result of configurations in which the secondary body is repelled from the primary shock due to shock impingement. Note also that this increase would be larger were these configurations not compensated for to some degree by those in which entrainment occurs, the effect of which is to make a single crater more likely. 


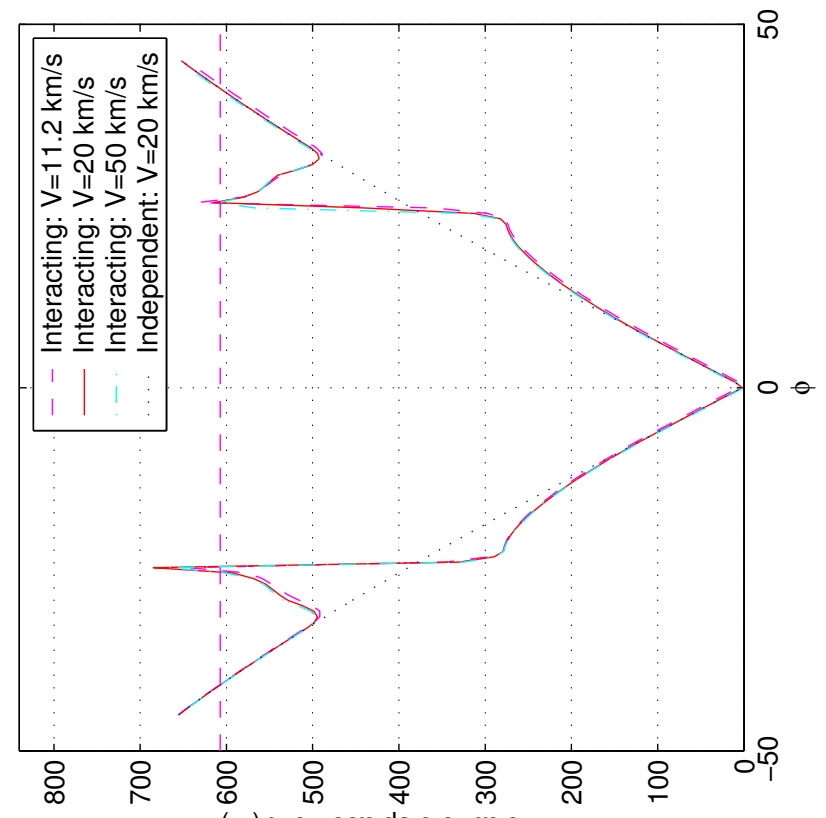

(u) ұuәшәэе|ds!p әм!ฺе|әу

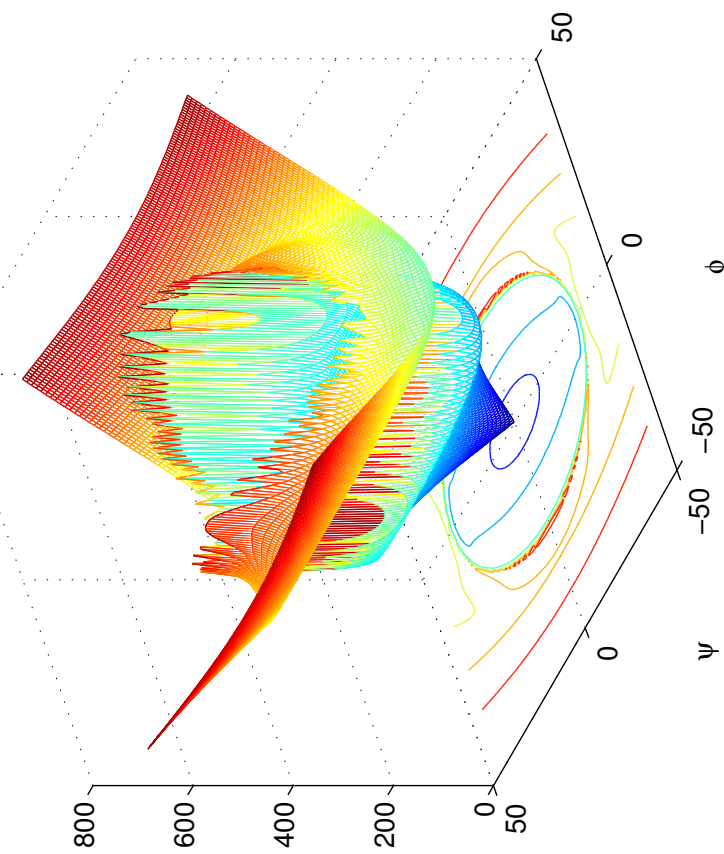

(w) łuәшәэе|ds!̣ әм!̣е|әч $\nsubseteq \nsubseteq$

4 웅

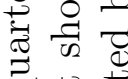

E

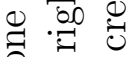

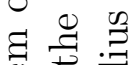

近宁

is 0

$\lambda \stackrel{\frac{\pi}{0}}{\stackrel{\Phi}{\sigma}}$

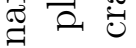

으 엉

ठ $E$

पै $\dot{0}$ :

릴

부의

둰

फै चै

피 굥

¿-

$-\frac{1}{0}$ के

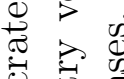

ن 节

芯

శ్ శ్తి

ㄹ. 일

- ฮิ ป

$\forall . \exists$

항

넝

$\rightarrow \frac{0}{\infty}$

芯

过

屯

苨它.

잉ㅇㅇ

胥

氙

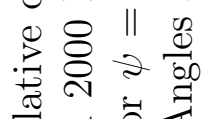

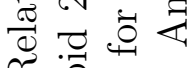

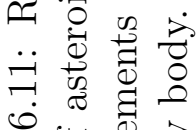

0 แ艹

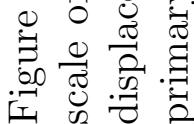




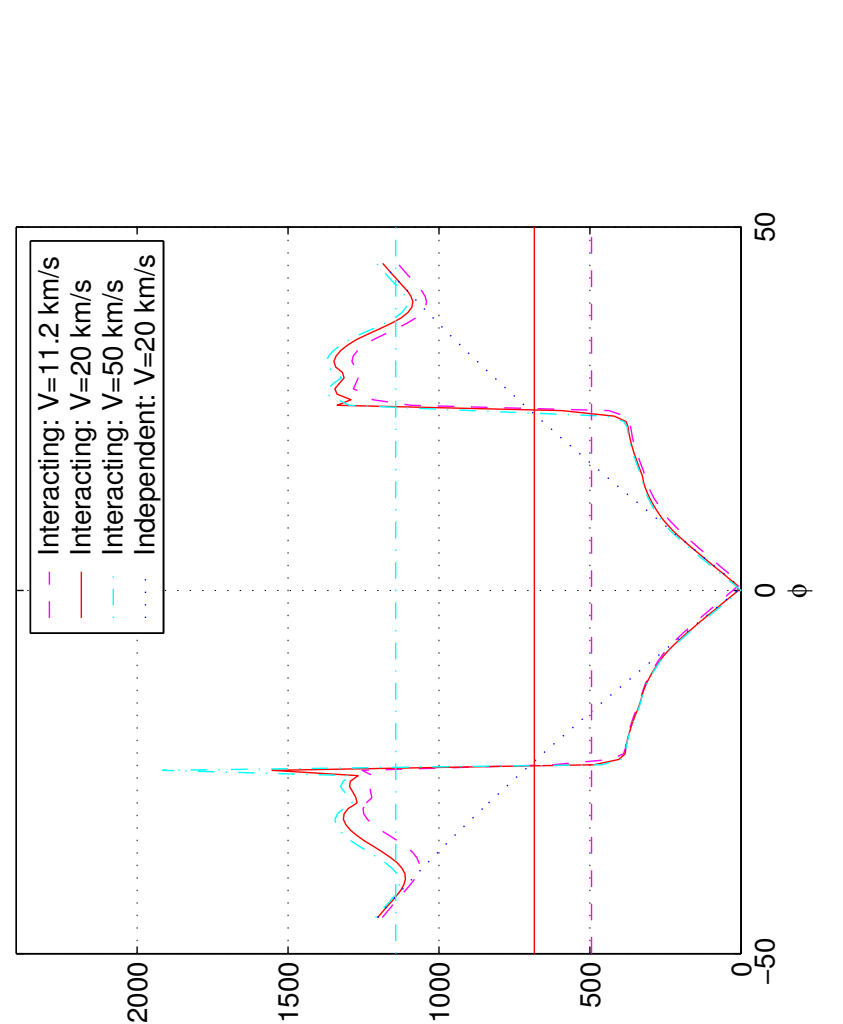

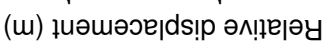

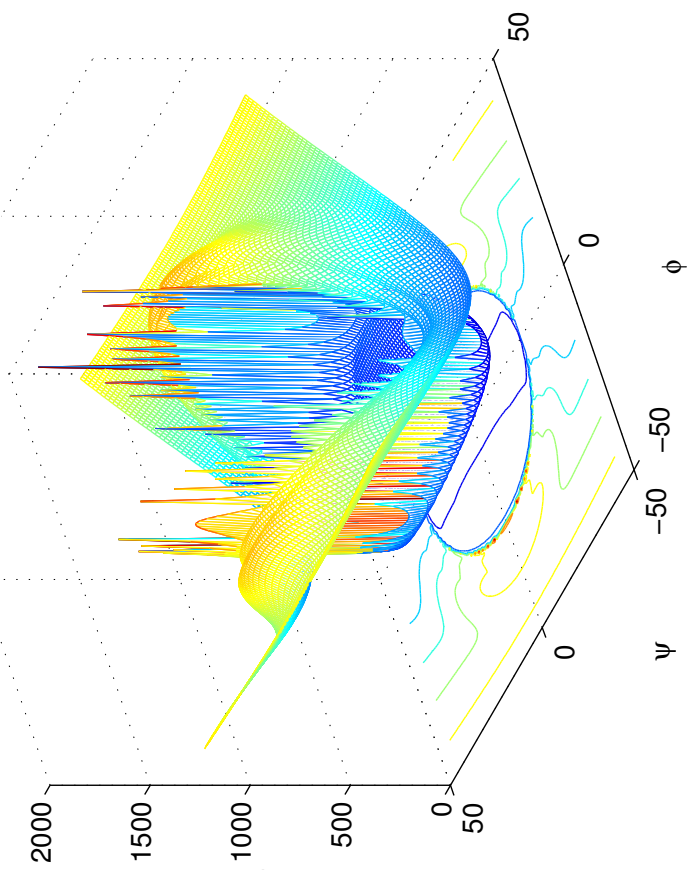

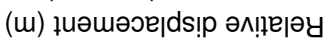

$\nsubseteq \nsubseteq$

훙

च च

\&

. .000

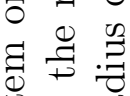

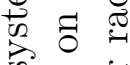

क ज एँ

$\overrightarrow{0}+\frac{1}{\sigma}$

氝

ల్త

ब $\mathrm{E} .0$

पै $\dot{0}$

हु छ

그의

곤

तี 0

피 离

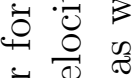

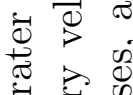

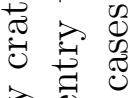

需

㞼

च्च

○

¿ $\frac{\infty}{5} \cdot$

ॠु

$\rightarrow 0$

苂

깅

仓

40

ॠ

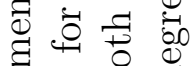

잉 응

完 0.

吾 คि

$.8 \delta \frac{0}{2}$

焉

Ð $\circlearrowright$

둘

0

$\exists 00$

के तु 


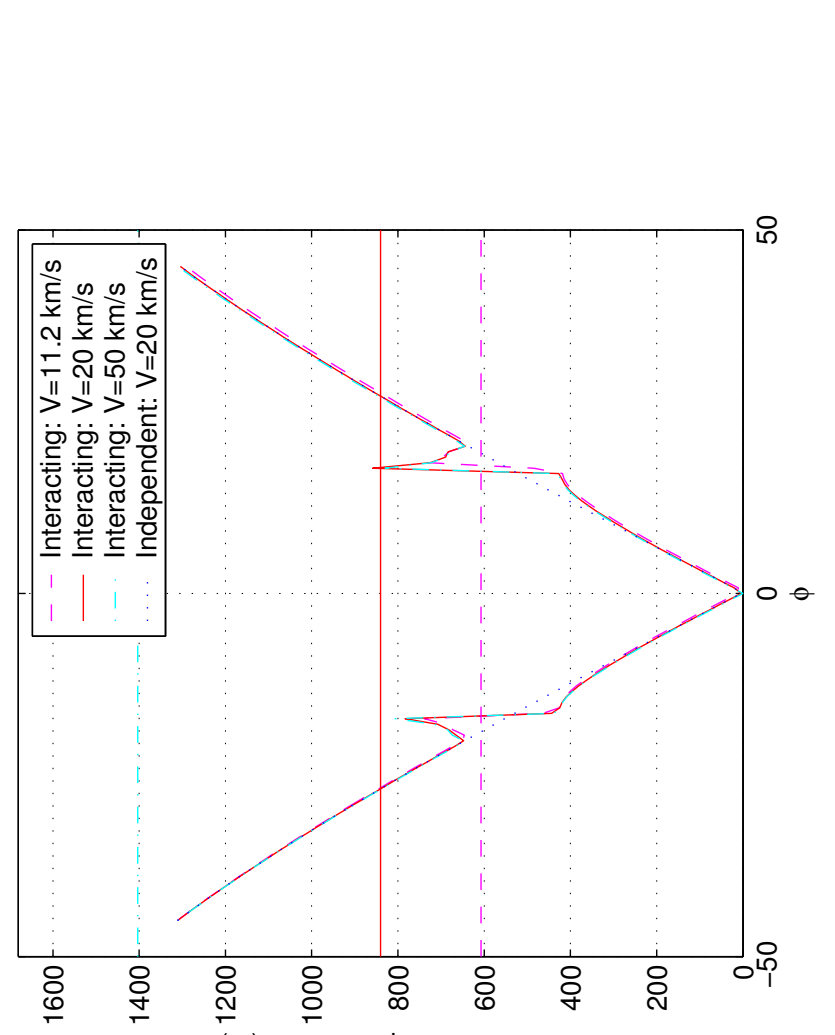

(u) јиәшәэе|ds!़ әм!ฺе|әу

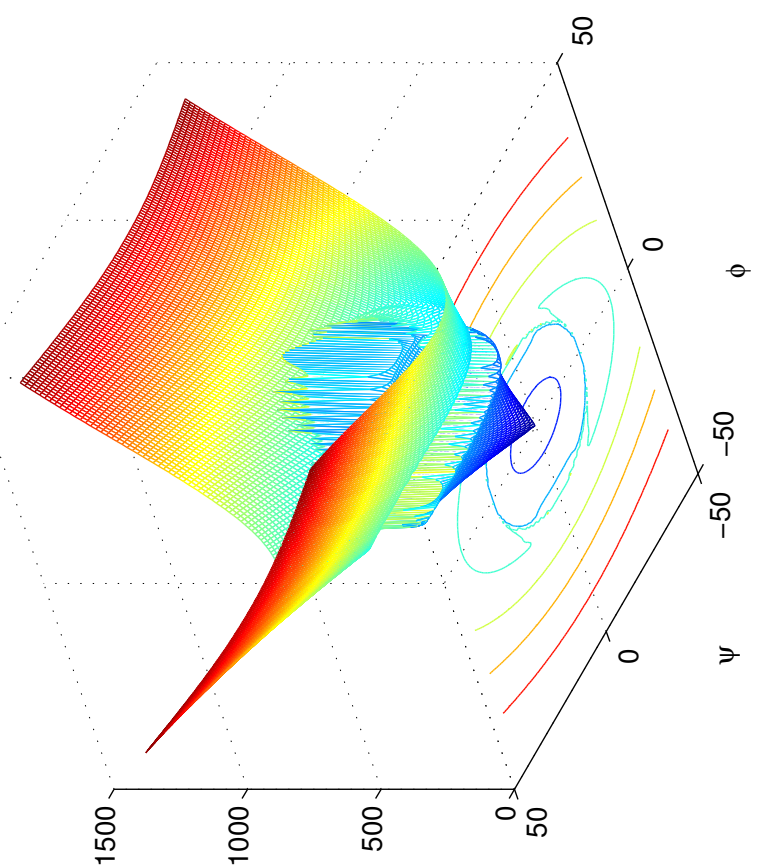

(u) ұиәшәэе|ds!̣p әл!ฺще|әу

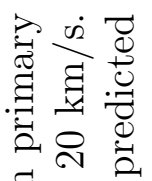

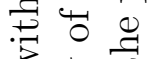

30

घ

क

긍

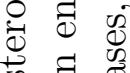

范

శี

嵌

둥

붕

동 $\frac{0}{60} 0$

范

도 $>$

总营

호

娄

要

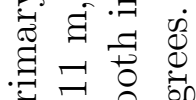

है

ㄱ

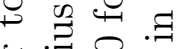

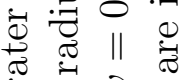

पु 2 क

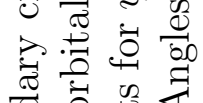

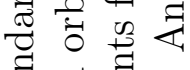

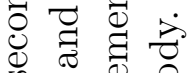

क

의

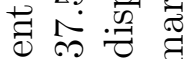

의

进 :

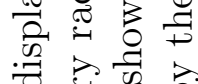

$\because$ 령

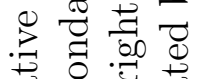

$\frac{\pi}{0} \quad 00 \pi$

क ज

@̈

잉 8

은

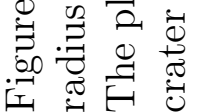



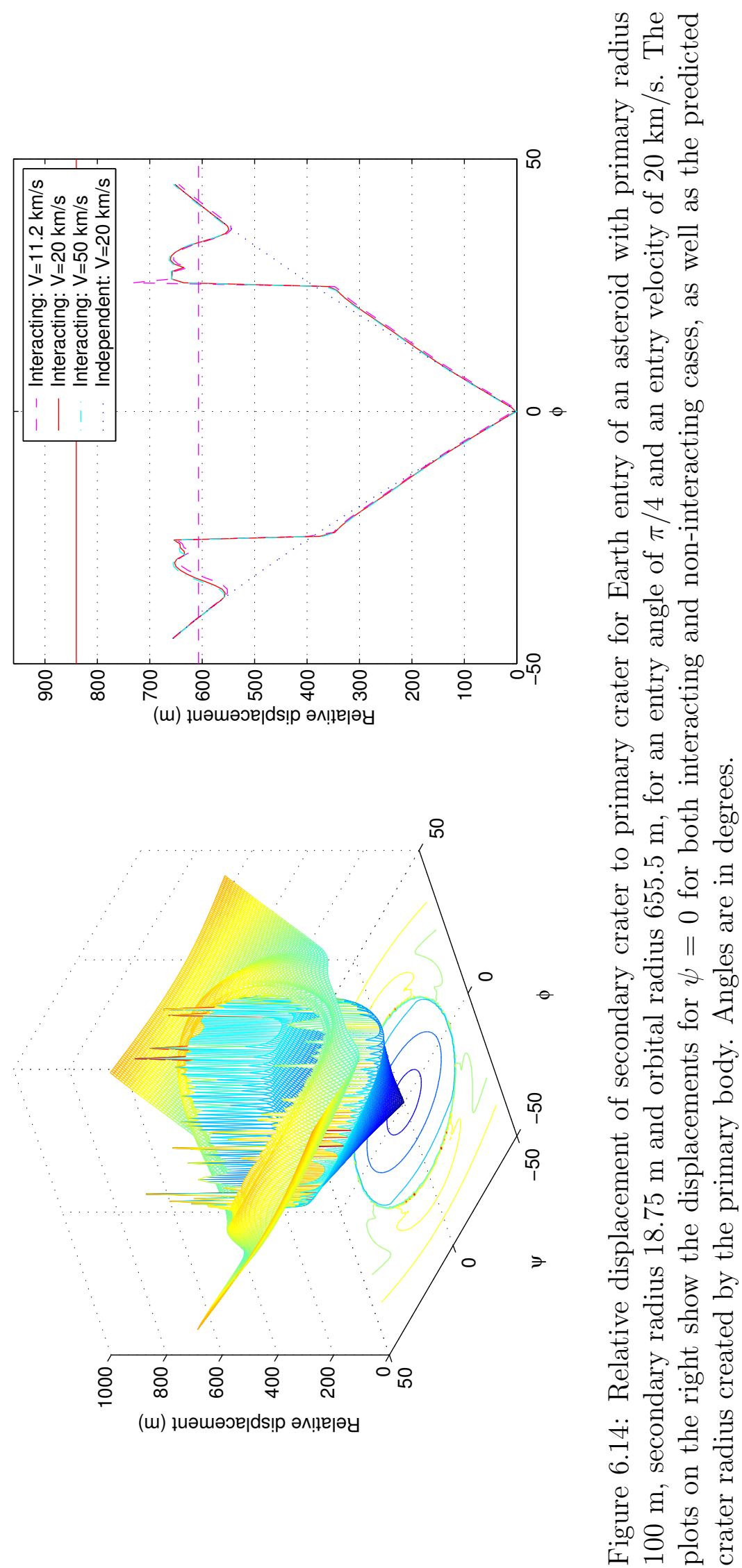


\section{Chapter 7}

\section{Meteoroid Fragmentation in a Planetary Atmosphere}

\subsection{Introduction}

It has been estimated that over 70 million meteoritic bodies enter the Earth's atmosphere every day (Baldwin, 1963). A vast majority of these burn up in the upper atmosphere, but occasionally a larger body will penetrate into the lower atmosphere. For bodies $\sim 1 \mathrm{~km}$ or larger in diameter, the pressure waves induced by the resulting aerodynamic loading do not have time to travel throughout the body during the atmospheric transit. These bodies effectively do not see the atmosphere, and thus arrive at the surface of the Earth relatively intact. For smaller bodies, however, the aerodynamic loading will typically exceed the strength of the body at some point during the flight, and breakup will occur. If the resulting fragments are large enough, they will survive transit through the remaining atmosphere and leave a crater field on the surface of the Earth. A recent example is the Sikhote-Alin meteoroid. The crater field produced by such a fragmentation event is typically elliptical in shape and is a record of the processes undergone by the fragments from the moment of breakup onwards.

Passey and Melosh (1980) have estimated that three main effects are responsible for the separation of fragments in crater fields: gravity/drag, bow shock interactions, 
and separation due to crushing. The first of these is well understood and is primarily responsible for the downrange separation seen in crater fields (i.e., along the semimajor axis of the ellipse). Smaller fragments are decelerated more quickly by aerodynamic drag than larger fragments, allowing gravity a greater time to steepen their trajectories. Larger fragments thus typically impact further downrange, with smaller fragments impacting towards the uprange end of the field. The other two effects are less well understood and are responsible primarily for the cross-range separation.

Within the the main scatter ellipses of more recent falls, however, are sometimes observed secondary patterns that are indicative of further processes at work. Krinov (1974), for example, identifies secondary scatter ellipses in the Sikhote-Alin field and attributes these to further stages of fragmentation. As was noted in Chapter 2, however, the effect of aerodynamic interactions after the main separation could result in a pattern similar to that that would be produced by secondary fragmentation. In particular, if a large fragment entrains several other fragments inside its shock, this could lead to a grouping of craters within the main scatter pattern.

In this chapter, then, we put forward the hypothesis that the secondary scatter ellipses seen in fields such as Sikhote-Alin are produced by later-time aerodynamic interactions rather than secondary fragmentation. To test this, we will carry out simulations of a Sikhote-Alin-like breakup and fall. A single fragmentation event will be assumed in each case, but during the fall the fragments will be allowed to interact aerodynamically. Comparisons will be made with simulations in which no aerodynamic interactions are included, and conclusions will be drawn based on patterns seen in the resulting fields. First, however, we will outline the models used in these simulations to describe the fragmentation event and the aerodynamic processes during the fall. 


\subsection{The Fragmentation Process}

The processes at work during the actual fragmentation of a meteoroid are not particularly well understood, and no attempt to further this understanding will be made here. For the current work, we are more concerned with modeling the aerodynamic interactions after the fragmentation event. However, to carry out such a modeling, we require at least a plausible initial arrangement of fragments with appropriate separation velocities. Producing such an arrangement is in itself not a trivial task and will be the focus of the current section.

\subsubsection{Generation of Fragment Masses}

As in the binary meteoroid case, assumptions will need to be made about the shapes of the parent body and fragments. For simplicity, the parent body is assumed to be spherical and unrotating, although these assumptions are not key to our model. The fragments are also assumed to be spherical, in this case because we wish to use the extended blast wave analogy of Section 6.3 to model the subsequent aerodynamic interactions. There is some justification to this last assumption, however. Fragmentation models, such as the dynamic fragmentation model of Grady (1982), often assume that the generation of fragments is such as to minimize the overall energy density with respect to the fracture surface area. Since, for a given fragment size, the surface energy to volume ratio is minimized in the case of a spherical fragment, we might expect the fragments to be generally spherical in shape.

It is assumed that a particular fragmentation event is to be modeled, and that the fragment number, which we denote by $N$, is known. To generate the fragments, $N-1$ random numbers between 0 and 1 are generated. These numbers are ordered, dividing the interval $[0,1]$ into $N$ fractional lengths, each of which is assigned to a fragment. The mass of each fragment is then taken to be the assigned fraction of the 
initial parent body mass. This process was presented by Grady and Kipp (1985) to describe dynamic fragmentation, but the differences between particle size statistics in static and dynamic fragmentatation are not well understood, and theoretical work in both areas has tended to start from similar statistical assumptions. It may be shown that as the parent body becomes large in relation to the average fragment size, the resulting distribution of masses is described by Poissonian statistics (Grady and Kipp, 1985).

\subsubsection{Initial Fragment Positions}

Having generated the fragment masses, we wish to position them within the parent volume in an appropriate manner. In general, it is impossible to fill a given volume with an arbitrary distribution of spheres without either some empty remaining volume or some overlap between spheres. If the sum of volumes of the spheres is the same as the total volume of the space to be filled, as is the case here, the unoccupied volume will be equal to the overlapping volume, and we seek to minimize this in some way. The approach taken here is to use an iterative routine, as follows.

The fragments are initially assigned positions at random within the volume of the parent body. If two spheres share an overlapping volume, they are each assigned a "force" away from one another, with a magnitude proportional to the volume overlapped. For two spheres of radii $r_{1}$ and $r_{2}$, with centers separated by a distance $d<r_{1}+r_{2}$, it may be shown (see http://mathworld.wolfram.com/ Sphere-SphereIntersection.html) that the overlapping volume is given by

$$
V=\frac{\pi}{12 d}\left(r_{1}+r_{2}-d\right)^{2}\left(d^{2}+2 d\left(r_{1}+r_{2}\right)-3\left(r_{1}-r_{2}\right)^{2}\right)
$$

Once each pair of spheres has been considered, each sphere is displaced by an amount proportional to, and in the direction of, the sum of forces acting upon it. If 
this leads to part of a sphere being outside the primary volume, it is brought back inside. This process is repeated until the sphere positions converge to a somewhat steady arrangement (some small oscillations will be unavoidable).

Figure 7.1 shows one example of this approach. The number of fragments is 200 , and the radius of the parent body is 2 . The fraction of the total volume left unoccupied is estimated with a Monte-Carlo integration scheme. The fraction of the total volume left unoccupied before arrangement in this instance is 0.425 ; this value is reduced to 0.157 by the arrangement.

To investigate the effect that the number of fragments has on the efficacy of this arrangement process, a number of simulations were run for a variety of fragment numbers (100 simulations for each choice of fragment number) and the mean reduction factors calculated. The results from these simulations are shown in Figure 7.2. The upper plot shows the mean of the volumes left unoccupied before and after the arrangement process - in each case this was again estimated using a Monte-Carlo integration scheme. The reduction factor may be seen to be typically of the order of 2.5-3. The lower plot shows the sum of all intersected volumes between pairs of spheres, divided by the sum of fragment volumes, before and after arrangement. As the same physical volume may be occupied by more than two spheres, it is possible for this value to exceed 1, as indeed may be seen to happen. The reduction factor for this value is greater - typically $4-6$. Note that a minimum occurs in both reduction factors at around 500 fragments.

\subsubsection{Fragment Separation Velocities}

We now turn to the velocities the fragments might be expected to have after the fragmentation event and initial separation phase. Let us first consider the source of these velocities.

For a meteoroid of a few to a few tens of meters in diameter, the aerodynamic 

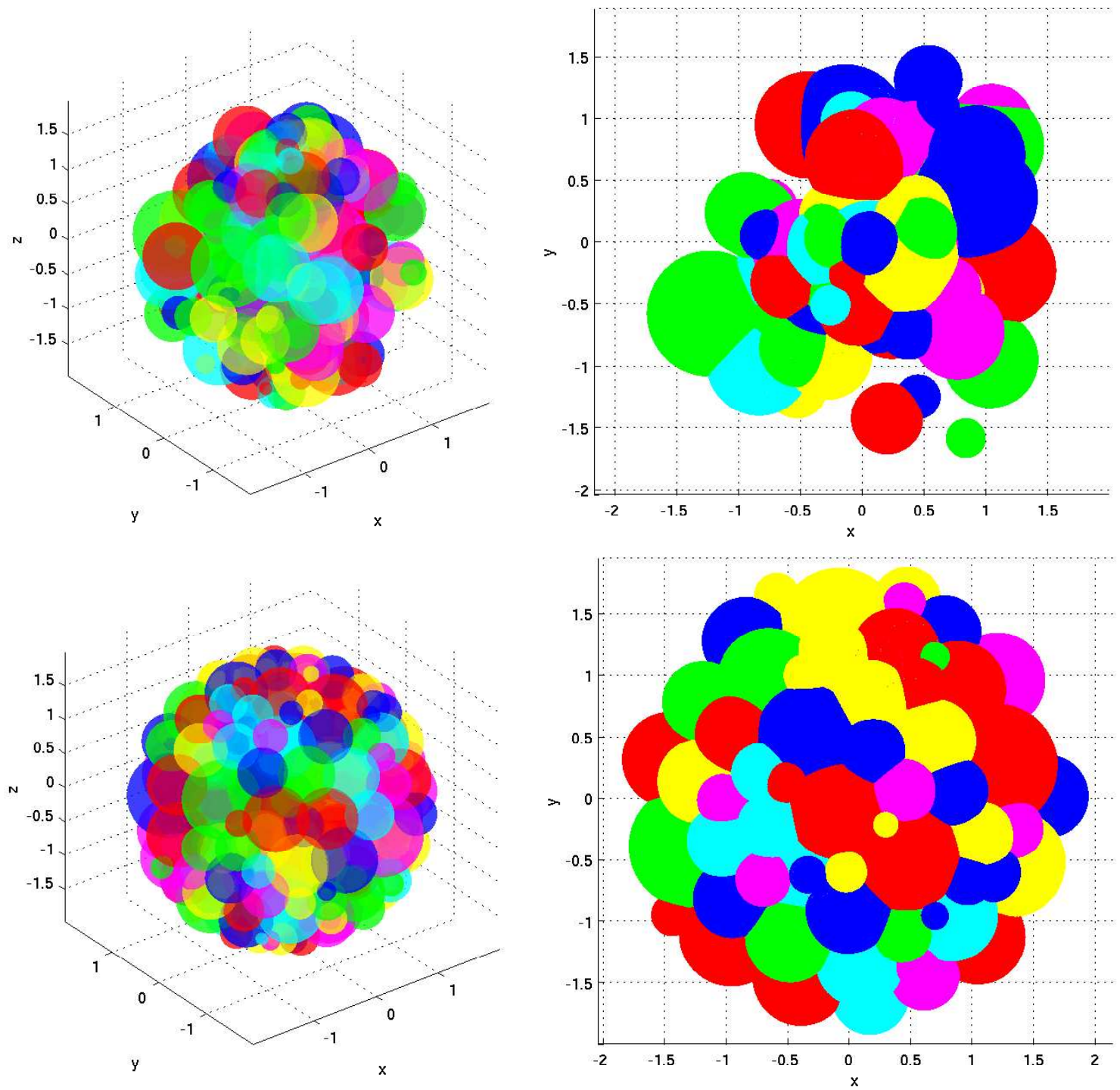

Figure 7.1: Configuration of spherical fragments before (top) and after (below) arrangement according to the procedure described. The left plot in each case shows an exterior view of the fragments, while the right plot shows a cut through the center of the initial volume. The number of fragments is 200 . 

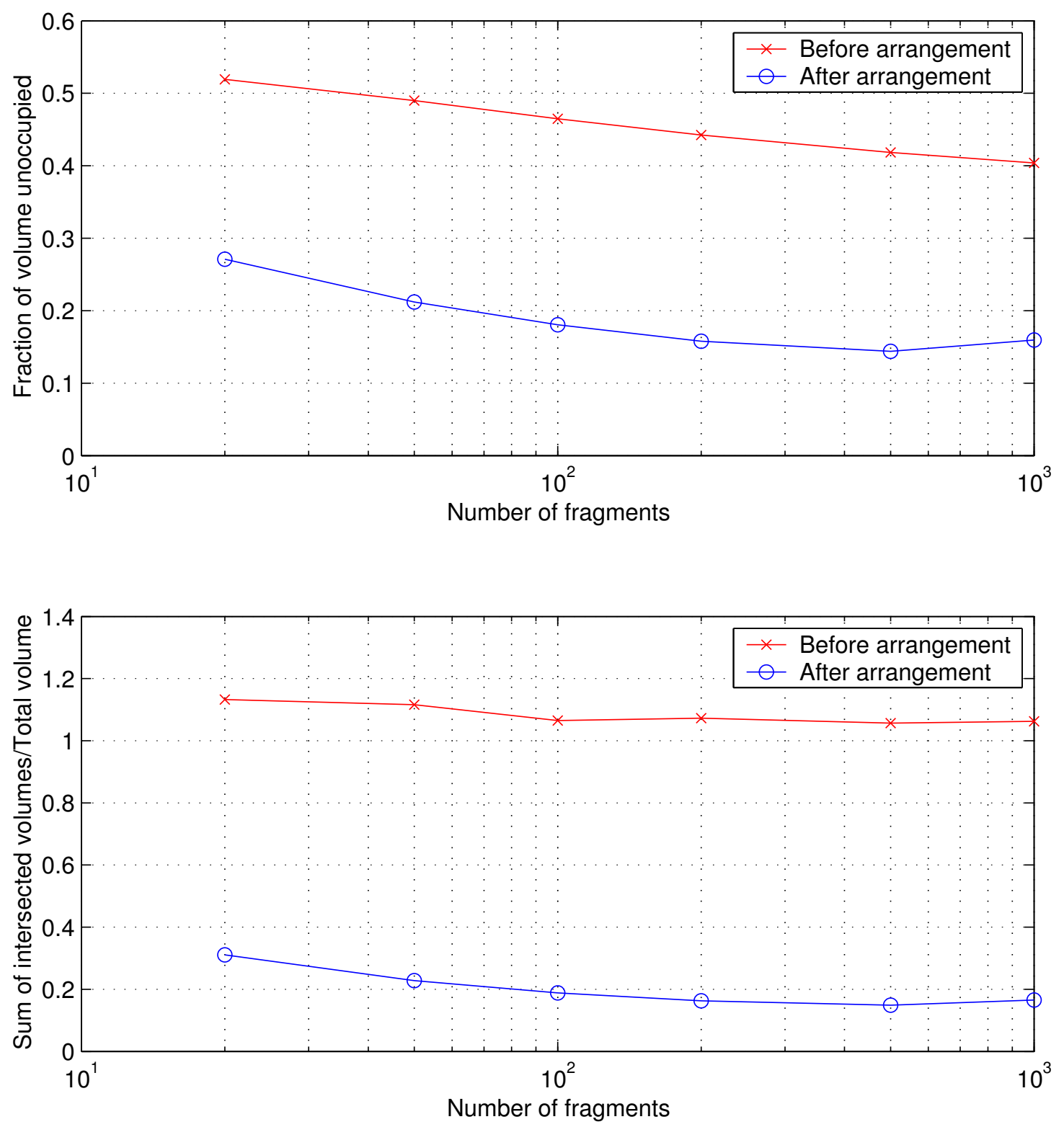

Figure 7.2: Reduction in the unoccupied volume (top) and the sum of intersecting volumes (bottom) divided by total volume for various numbers of fragments. 
loading experienced during the atmospheric transit will be, to a good approximation, quasistatic. Consider, for example, a body $20 \mathrm{~m}$ in diameter entering the atmosphere at a rather high velocity of $50 \mathrm{~km} / \mathrm{s}$ at the most probable entry angle of $\pi / 4$. Assuming a sound speed of $4 \mathrm{~km} / \mathrm{s}$ in the body, a compression wave will take 0.01 seconds to travel across the body and be reflected back again. During this time, the body will have travelled $500 \mathrm{~m}$, or $350 \mathrm{~m}$ in the vertical direction. Assuming an atmospheric scale height of $8.4 \mathrm{~km}$, the atmospheric density will have increased over this time by $4 \%$. As the velocity will change very little during this time, this will also be the increase in dynamic pressure. As this increase is small, the aerodynamic loading may be treated as approximately quasistatic during the transit.

Given that the loading is static, the kinetic energy imparted on the fragments during the fragmentation event can only come from the strain energy within the meteoroid just prior to fragmentation. To estimate the mean fragment velocity resulting from this, we note that the strain energy $\mathcal{E}_{s}$ in the unfragmented meteoroid may be estimated as

$$
\begin{aligned}
\mathcal{E}_{s} & \approx \bar{p} A \Delta l \\
& =\bar{p} A l e \\
& =\bar{p} A l \frac{\sigma}{E} \\
& =\frac{\bar{p}^{2} A l}{E},
\end{aligned}
$$

where $\bar{p}$ is the mean pressure over the front of the sphere, which we may estimate as $\rho_{a} V^{2} / 2, \rho_{a}$ being the atmospheric density; $A$ is the meteoroid cross-sectional area; $l$ the length; $e$ and $\sigma$ the strain and stress, respectively; and $E$ the Young's modulus. For an iron meteoroid, with Young's modulus $\approx 200 \mathrm{GPa}$, of $\sim 2 \mathrm{~m}$ diameter, fragmenting at a height of $15 \mathrm{~km}$ and a velocity of $20 \mathrm{~km} / \mathrm{s}$ (these will be the approximate values of the meteoroid we will consider shortly), the strain energy will be $\sim 20$ MJ. 
Assuming that this is converted entirely into kinetic energy of the fragments, the mean fragment velocity will be given by $v_{f}=\sqrt{2 \mathcal{E}_{s} / m}$, where $m$ is the meteoroid mass. In the current case, $v_{f} \sim 1 \mathrm{~m} / \mathrm{s}$, which is very small.

The fragmentation process will thus impart only a negligible initial velocity on the fragments; the bulk of the separation velocity must come from the repulsive aerodynamic forces developed as the fragments separate. This force is present because in the space between two closely positioned fragments the pressure is close to the dynamic pressure, $\rho V^{2}$, whereas on the outsides of the bodies the pressures are much smaller. Passey and Melosh (1980) deduced that for two fragments, the tranverse velocity developed due to this interaction is

$$
V_{T}=\left(\frac{3}{2} C \frac{R_{i}}{R_{f}} \frac{\rho_{a}}{\rho_{m}}\right)^{1 / 2} V_{i}
$$

where $R_{i}$ and $R_{f}$ are the radii of the initial meteoroid and fragment, respectively, $\rho_{a}$ and $\rho_{m}$ are the atmospheric and meteoroid densities, and $V_{i}$ is the initial meteoroid velocity. The value of the constant $C$ was deduced from the cross-range dispersions in known crater fields to lie between between 0.02 and 1.5 .

For the case of many fragments, however, the situation is somewhat more complicated, and a simple analysis such as the one that resulted in Equation 7.2 is not possible. Note, for example, that the tranverse velocity in this expression is greater for smaller fragments. This effect will tend to be neutralised in the many-fragment case by the fact that the pressure difference from one side of the fragment to the other that is responsible for the repulsive force will be greater in the case of a larger fragment.

Given the large degree of uncertainty involved in determining the fragment velocities resulting from the initial aerodynamic interactions, then, we will assume that initial velocities are imparted on the fragments as follows. Each fragment is assumed 
to develop a radial velocity away from the center of the parent body, with a magnitude proportional to the initial displacement from the center. This is similar to the dynamic fragmentation model of Grady (1982), but here is assumed to result from the aerodynamic interactions. The maximum velocity (i.e., that of the outermost fragments) is assumed to be proportional to the initial meteoroid velocity and the square root of the ratio of atmospheric density at the height of breakup to the meteoroid density, i.e.,

$$
V_{\text {max }}=C \sqrt{\frac{\rho_{a}}{\rho_{m}}} V_{i} .
$$

These dependencies come from Equation 7.2 and are also plausible in the manyfragment case. The constant of proportionality, $C$, is calculated to give the desired cross-range dispersion of the crater field in question.

Starting from their initial positions, the calculation of which was described in the previous section, the fragments are assumed to maintain this initial velocity until they achieve a certain average spacing, at which point aerodynamic modeling is initiated. For the current simulations, this crossover point occurs when the outermost fragments have reached a radius twice the initial radius of the parent body.

\subsection{Modeling of Aerodynamic Interactions}

The basis of the aerodynamic modeling is the procedure developed for the simulation of the atmospheric entry of binary meteoroids, as described in Chapter 6. Considering the more complex nature of the current problem, however, there are further assumptions that will have to be made in this methodology to enable it to be used here.

First, this model was tested only for cases in which the secondary body inside the shocked region was smaller than the primary body producing this region. We do not expect that the model will perform well in the opposite situtation. Thus, in 
our simulations, if a larger body is found to lie within the shock of a smaller body, they shall both be assumed to travel independently of one another. This is not a particularly restrictive assumption, since smaller bodies are more greatly affected by aerodynamic drag, and so the larger body would be expected to soon move ahead of the smaller body anyway.

Further, the methodology was developed to model binary interactions, and cannot be easily extended to the multiple-body case. We shall thus be limited to pair-wise interactions. If a smaller fragment lies within two or more shocked regions created by larger fragments, we consider only the effect from the largest fragment. Such multiple interactions might be expected to occur at very early times, but will be rare once the fragments have separated further.

In Chapter 6, as the two meteoroids remained relatively close throughout the atmospheric transit, we did not have to deal with the problem that, in the farfield, the conditions in the blast wave analogy do not tend towards the physical freestream conditions (as may be observed in Figure 6.2). In the present simulations this problem will become relevant, so, to prevent one fragment significantly affecting another extremely distant fragment, we set a downstream limit to the extent of a primary shock. The specification of the limiting value is somewhat arbitrary: we choose the point at which the Mach number immediately behind the blast wave shock, given by Equation 6.1, is equal to the freestream value.

There is also the possibility that, during a simulation, two fragments approach sufficiently close to one another that a collision would occur in the physical situtation. To avoid the added complication that collisions would introduce, we assume in our modeling that the fragments have zero cross-sectional area with respect to collisions. If the secondary fragment is found to lie within the primary shock, but ahead of the primary body, it is assumed to travel independently of the primary body.

In the case of the binary meteoroids, the velocities changed relatively little over 
the course of the atmospheric transit. For the smaller fragments considered here, however, the aerodynamic forces will greatly reduce the velocities over the course of the flight. Thus, the hypersonic drag coefficient of 0.88 will not be appropriate at all times. As the Mach number is decreased from large values, the drag coefficient of a sphere rises to a value of around 1 at $M \sim 1$ and then decreases to around 0.5 when the flow becomes subsonic. For the purposes of these simulations, we shall simply assume values of 0.88 for $M>1$ and 0.5 for $M<1$.

Finally, an adaptive time-stepping mechanism, based on the maximum relative speed between fragments, is implemented. This is to prevent the incidence of a fragment entirely crossing the shocked region created by another fragment within a single time-step.

A flowchart showing the sequence of events during each fragmentation simulation is shown in Figure 7.3.

\subsection{The Sikhote-Alin Crater Field}

In the present work, we will focus on the Sikhote-Alin crater field, located in Siberia, Russia. The fall that produced this field occurred on February 12, 1947, and because of its recent nature, many smaller craters are present that might have eroded away in the case of an older field. Over 150 craters are present in the field, as well as thousands of meteoritic fragments. The cross-range width is $0.9 \mathrm{~km}$, and the downrange length is $2.0 \mathrm{~km}$. Krinov (1974) noted the presence of secondary scatter ellipses superposed on the main ellipse and attributed these to secondary fragmentation. The impact angle has been estimated to be $30^{\circ}$ (Krinov, 1966), which provides an upper limit for the entry angle, as the combined effects of gravity and drag will steepen the angle of travel with respect to the horizontal.

The density of the meteoroid is taken to be $7.8 \times 10^{3} \mathrm{~kg} / \mathrm{m}^{3}$; a value appropriate 


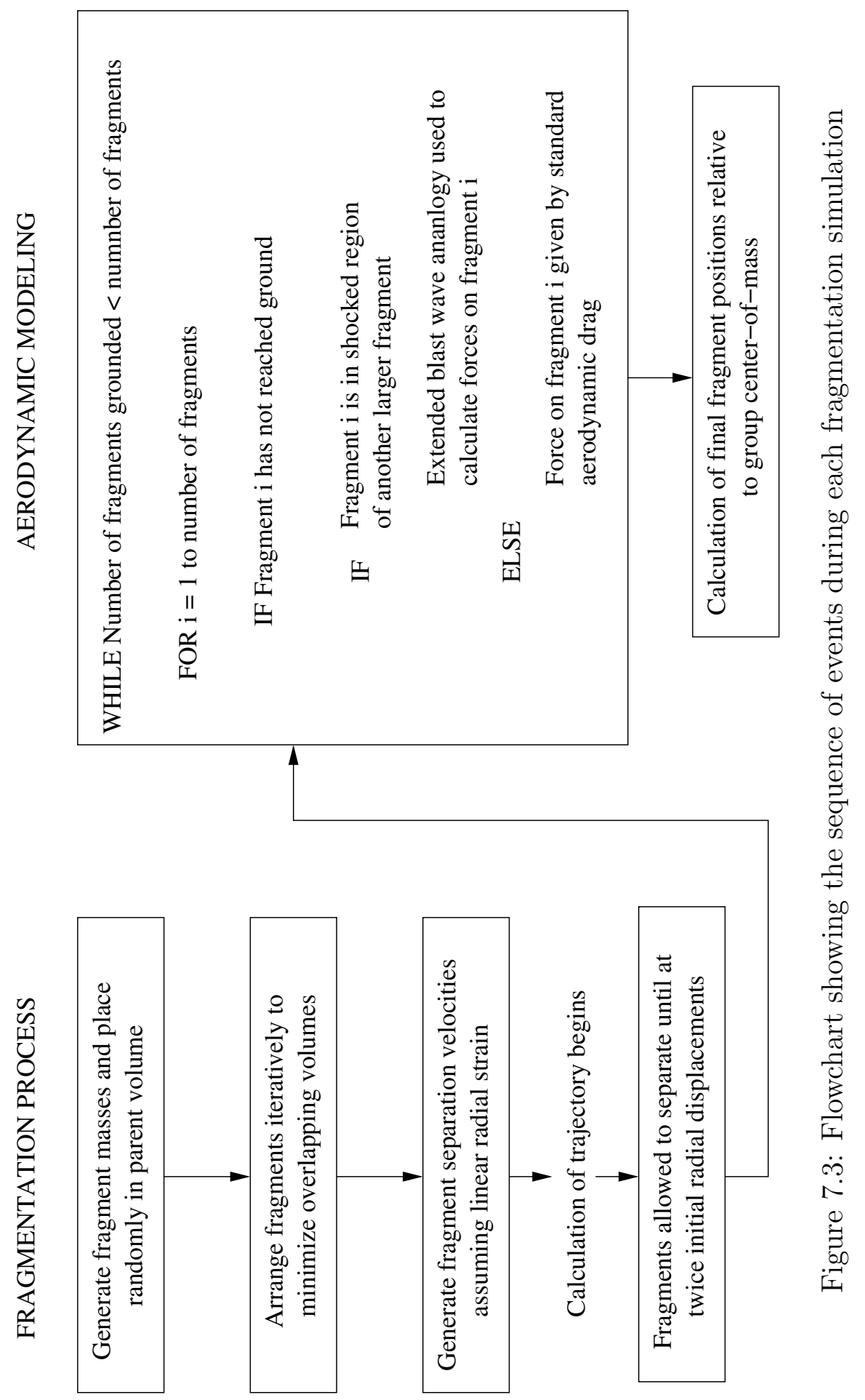


for an iron meteoroid. The total mass of the fall has been estimated at 70 tons (Krinov, 1966), with another 200 tons estimated to be present in the smoke trail (Heide, 1963). As we are not considering the effects of ablation in the current work, we will take the intermediate value of 200 tons as the initial mass, which gives a radius of approximately $1.8 \mathrm{~m}$.

Krinov (1966) estimated that the meteoroid entry velocity was $14-15 \mathrm{~km} / \mathrm{s}$, with the initial breakup occurring at high altitude and the final stage of fragmentation taking place at approximately $6 \mathrm{~km}$. More recently, Passey and Melosh (1980) have suggested that the initial fragmentation took place at $40 \mathrm{~km}$, which would require an entry angle of less than $20^{\circ}$, according to their model. In simulations in the current work, however, it was found that these values led to far greater downrange spread than is observed in the physical field. The problem may well be that Passey and Melosh use a drag coefficient value of 0.5 for the entire flight. This value is inappropriate at high Mach numbers and would lead to smaller fragments being less affected by drag and thus impacting further downrange.

Given the uncertainty in the values cited by the previous authors, a series of simulations was performed in order to determine appropriate values for the entry velocity, the fragmentation height, and the constant $C$ in Equation 7.3. An entry angle of $30^{\circ}$, corresponding to the estimated impact angle, was assumed, as the flight angles of larger fragments change relatively little during the flight. The fragmentation event was assumed to produce 200 fragments according to the procedure described above, but the smallest $10 \%$ of fragments were not considered. The reasoning for this was that these fragments would likely be ablated away during the remaining atmospheric transit, and even if they did survive, would typically be carrying insufficient speeds at impact to create discernible craters. A combination of a velocity and an altitude at fragmentation of $18 \mathrm{~km} / \mathrm{s}$ and $15 \mathrm{~km}$, together with a value for the constant $C$ of $\sim 3$, were found in general to lead to appropriate downrange and cross-range field 
lengths, although other combinations may be possible. These values will be used in the simulations in the following sections of this chapter.

\subsection{Results}

\subsubsection{Conditions During Aerodynamic Interactions}

First, having noted the limitations of the blast wave methodology in Chapter 6, we will investigate whether the aerodynamic interactions in the fragmenting meteoroid simulations are taking place at conditions at which we can expect the methodology to give reasonable results. In Figure 7.4 are histograms showing the relevant conditions for the aerodynamic interactions during a typical simulation. Shown are the Mach number of the primary body, the normalized downstream distance of the secondary, $x / d_{1}$, and the normalized speed difference between the two fragments $\left\|\mathbf{V}_{\mathbf{1}}-\mathbf{V}_{\mathbf{2}}\right\| / V_{1}$. The interactions shown are those from every tenth timestep and can be considered representative of all interactions calculated during the simulation.

The majority of interactions are seen to take place at a primary Mach number greater than 50, with only a small fraction occurring at a Mach number of less than 40. The minimum Mach number is approximately 25. The downstream distance for the interactions is typically at an $x / d_{1}$ value of less than 30 , with a small number at $x / d_{1}>50$. In Chapter 6 we concluded that at a primary Mach number of 50, we could expect the unmodified blast wave methodology to give reasonable results out to a downstream value of $x / d_{1} \sim 25$. Given the modifications that were subsequently made in order to increase this domain of validity, and also considering that the average primary Mach number is greater than 50, we may feel confident in extending this domain somewhat. We also see that the vast majority of interactions take place at a velocity difference of less than $5 \%$, which provides support for our assumption that the bodies are stationary relative to one another during interactions. We conclude 

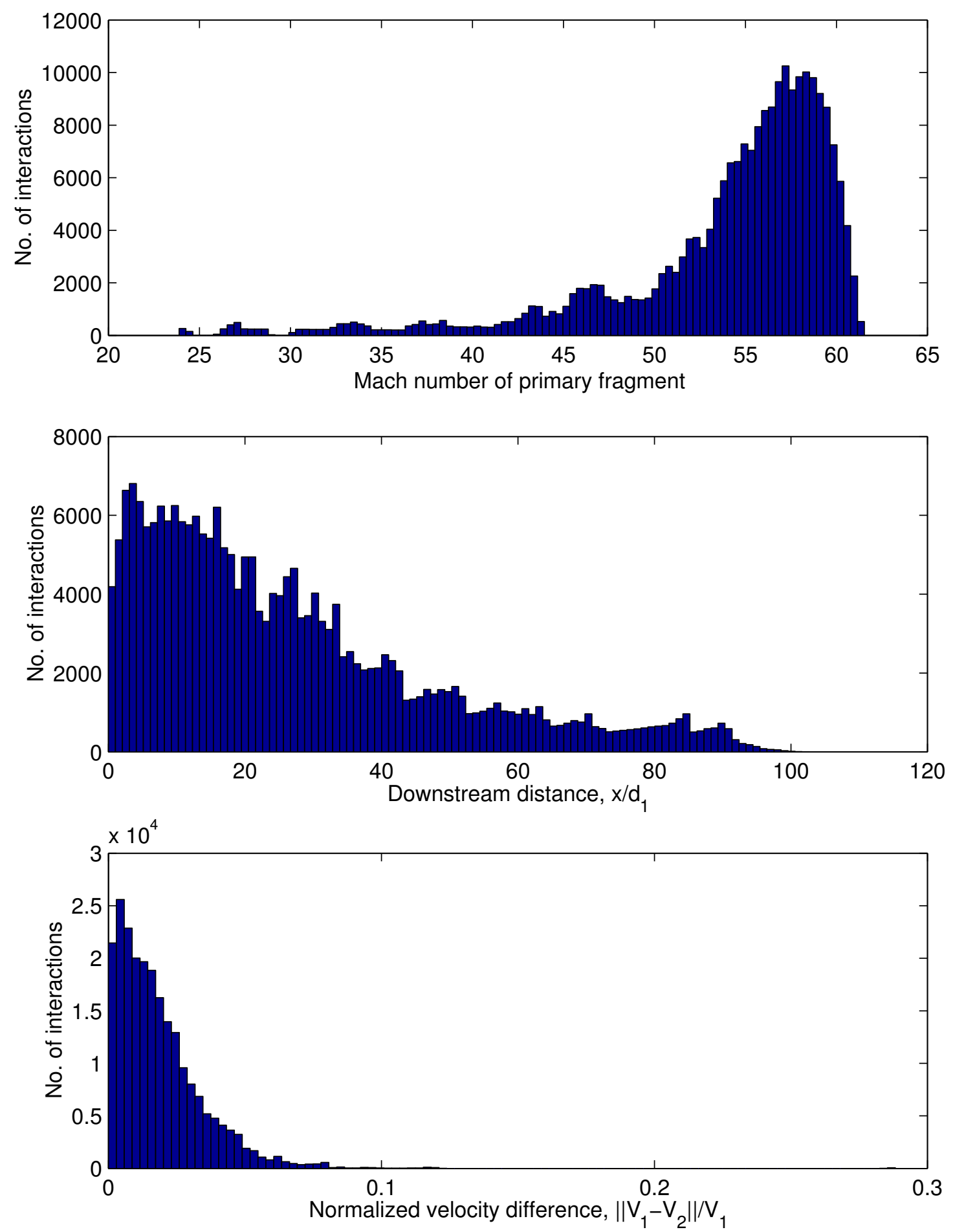

Figure 7.4: Histograms of primary Mach number (top), downstream displacement $x / d_{1}$ (middle), and relative fragment speeds $\left\|\mathbf{V}_{\mathbf{1}}-\mathbf{V}_{\mathbf{2}}\right\| / V_{1}$ (bottom) for aerodynamic interactions during a typical simulation. 
then that a substantial majority of the interactions take place at conditions at which we can be confident of the extended methodology giving reasonable results, although a few, in particular those at large downstream values, will be somewhat questionable.

\subsubsection{The Crater Field}

In Figures 7.5 and 7.6 are plotted the crater fields resulting from several fragmentation simulations. The left side in each case corresponds to the downrange end of the field. In order that the effect of the aerodynamic interactions may be gauged, crater locations are also plotted from simulations with identical initial conditions but in which all fragments are subsequently assumed to travel independently of one another. The crater radius, $R_{c}$, corresponding to each fragment is assumed to be a function of the impact kinetic energy, $E_{k}$, and the impact angle, $\theta_{f}$. The scaling law used here is similar to that considered by Passey and Melosh (1980) for craters up to 10m in diameter:

$$
R_{c} \sim E_{k}^{1 / 3} \sin ^{2 / 3} \theta_{f}
$$

where the constant of proportionality depends on the impactor and target densities. The crater radii are not, however, to the same length scale as the crater locations.

As may be seen, although the overall crater field shapes are not significantly altered by the aerodynamic interactions, the locations of fragments within the fields differ notably between the interacting and non-interacting cases. In particular, secondary groupings of craters appear more likely to occur in the interacting case, as we predicted earlier. In each field, the most notable secondary groupings that result from the interactions are indicated by dashed boundaries, although the selection of these is subjective to a small degree. The amount of secondary grouping does seem to vary somewhat from simulation to simulation, however. Note, for example, that in the top field in Figure 7.6, although there are many small groupings of craters resulting 

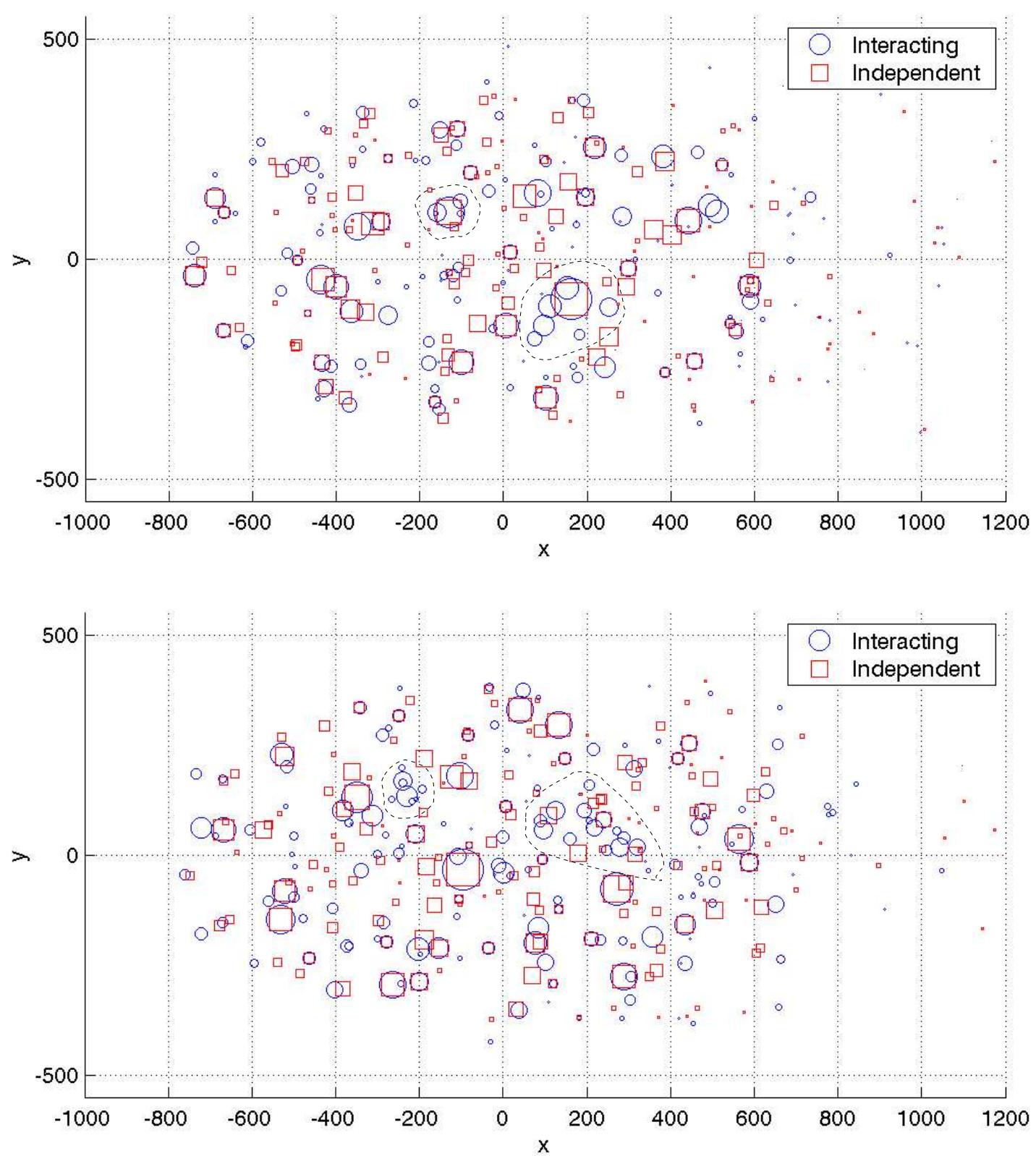

Figure 7.5: Crater locations for two simulated falls with and without aerodynamic interactions after separation. The origin corresponds to the center of mass of the system, with the left side corresponding to the downrange end of the field. Possible secondary groupings of fragments for the interacting cases are indicated by dashed boundaries. 

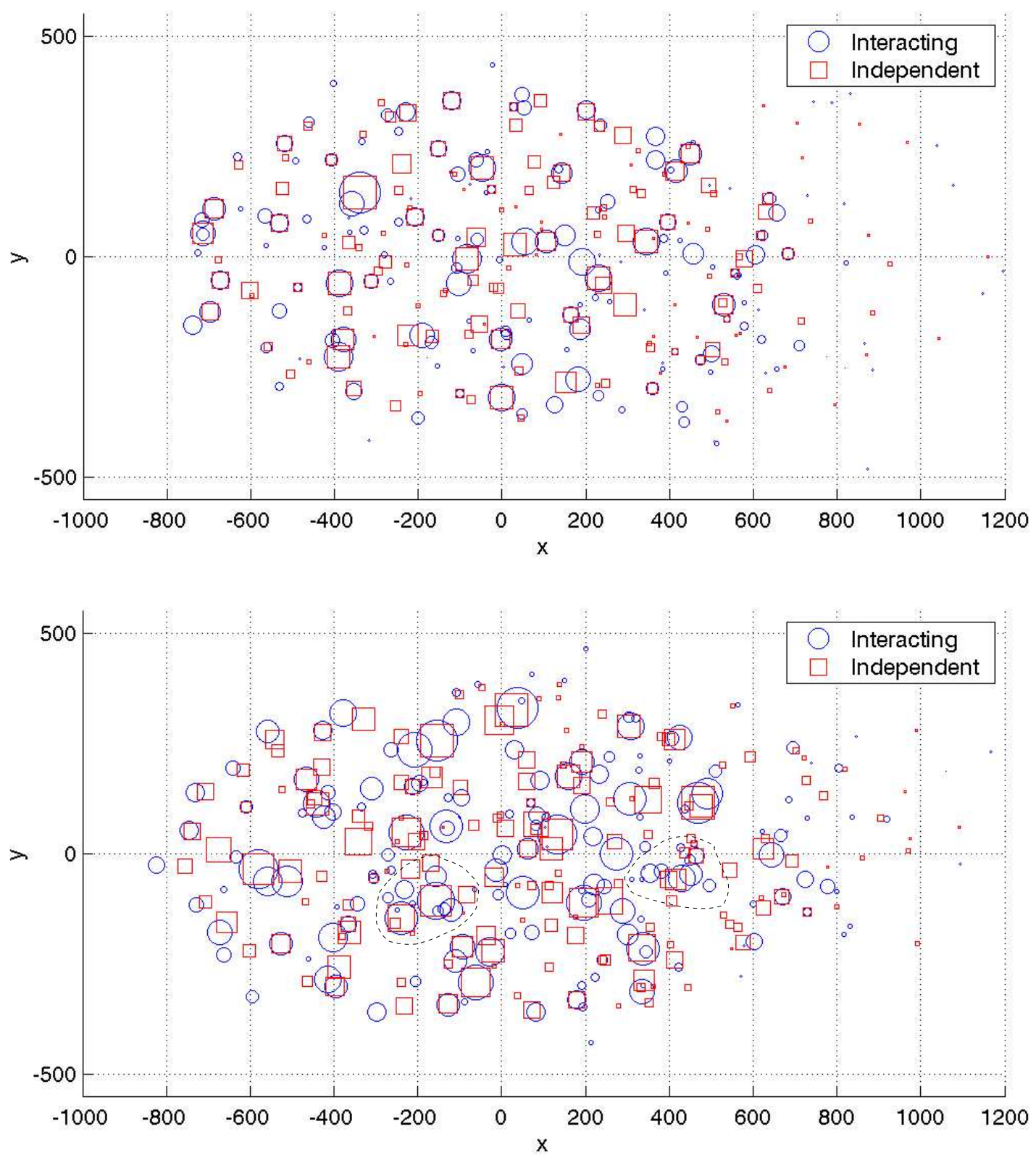

Figure 7.6: Crater locations for two further simulated falls 
from the aerodynamic interactions, there are no obvious larger-scale groupings such as may be seen in the other fields. This contrasts most obviously with the lower field in Figure 7.6 in which much large-scale grouping is seen, although only two of the more notable groups are indicated.

\subsubsection{Statistical Analysis of Crater Distributions}

\subsubsection{Overall Crater Field Shape}

In order to provide more substantive evidence that secondary grouping is more likely to occur in interacting simulations, a statistical analysis has been performed on a sample set of fifty simulations, comparing interacting and non-interacting cases. First, we will try to gain an overall picture of the fragment position distributions in the fields produced by these simulations, focusing in particular on how these positions might vary with fragment radius.

Figure 7.7 shows scatter plots of the downrange and cross-range displacements

of the combined fragments from the fifty simulated falls as functions of the fragment radius. Both interacting and non-interacting cases are shown. The fragment displacements in each case are calculated from the final position of the group centerof-mass for that particular simulation. In the upper plot we see the expected result that smaller fragments typically fall further uprange (indicated by a more positive downrange displacement value) than larger fragments due to the drag/gravity effect described earlier. However, the aerodynamic interactions are seen to result in a number of smaller fragments impacting further downrange than they would otherwise. This must be caused by entrainment of these fragments by larger fragments, which would negate to some degree the drag/gravity effect. Note, however, that this entrainment does not seem to be present in the case of the smallest fragments. A likely explanation for this is the body-size effect noted in Chapter 2 - a smaller fragment 
travelling in the shocked region of a much larger fragment will experience a repulsive lateral force and will thus not be entrained. Supporting this explanation is the fact that the ratio of the fragment radius below which the entrainment effect no longer appears significant $(\sim 0.09 \mathrm{~m})$ to the radius of a typical large fragment is approximately 1:6, which is the critical body-size ratio determined in Section 2.4.2.

In the lower plot of Figure 7.7, the aerodynamic interactions are also seen to result in some smaller fragments falling at more extreme cross-range displacements. This can be explained by a combination of the body-size effect and repulsion due to primary shock impingement, as described in Section 6.3.

In Figure 7.8 the downrange and cross-range displacements are again plotted, but in this case the fragments have been grouped into thirty equally spaced bins according to fragment radius, and the mean displacement and standard deviation for each bin is plotted (note that the absolute value of the cross-range displacement is considered here). Each data point is located on the $x$-axis at the maximum fragment radius for that bin, with the non-interacting cases shifted slightly for clarity. The differences in mean values between the interacting and non-interacting cases are as we may have predicted from Figure 7.7. For smaller fragments (but not the very smallest), the mean downrange displacement is less in the interacting case than in the noninteracting case, while the mean cross-range displacements for smaller fragments is increased by the aerodynamic interactions. Note that these differences all lie within the bin standard deviations, however.

\subsubsection{Crater Groupings and Pair-wise Statistics}

We now wish to consider the fragment distributions within each simulation in order to determine whether statistically significant differences in groupings may be seen between the interacting and non-interacting cases. To this end we will consider pairwise relative displacements of fragments within each fall pattern, as follows. 

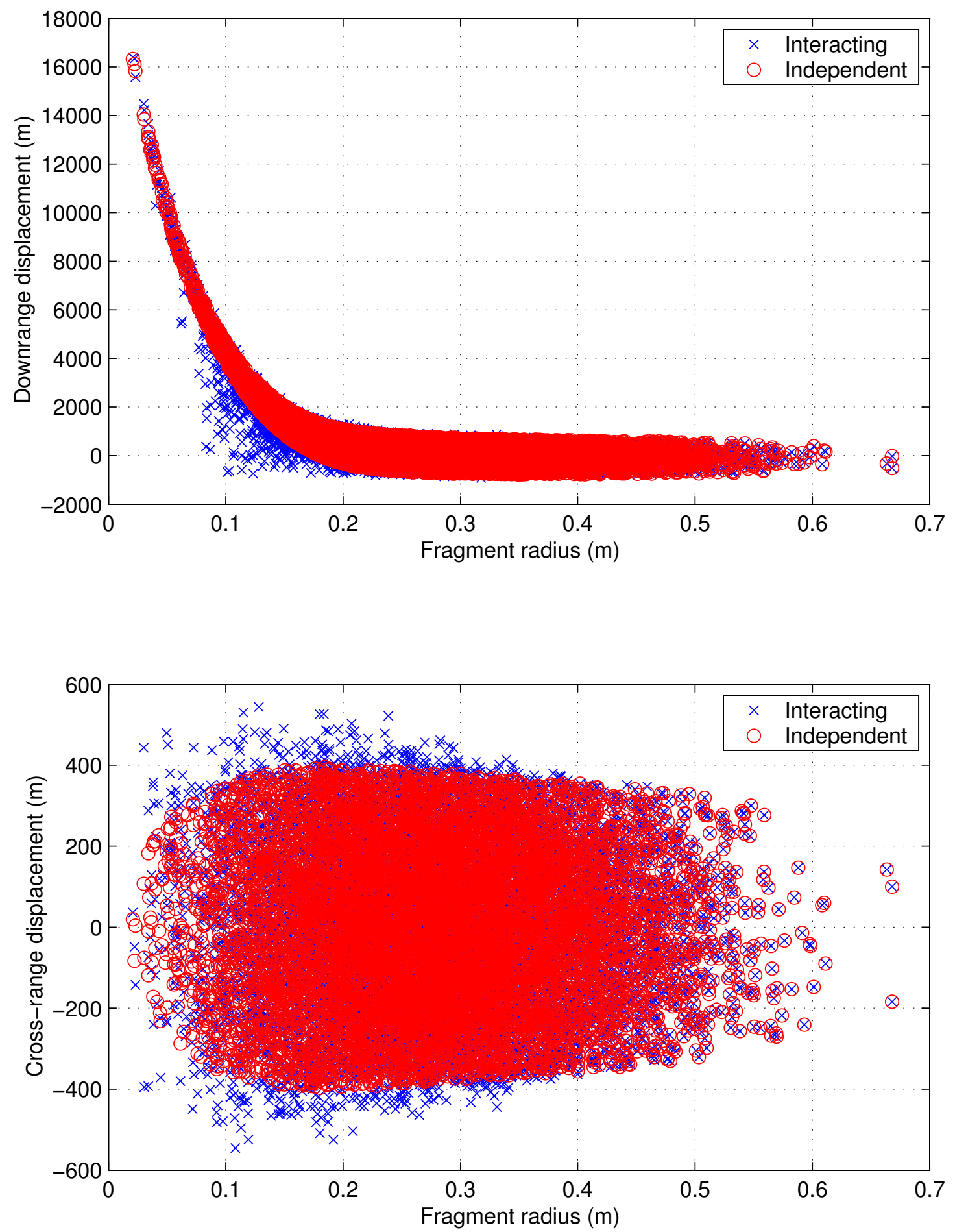

Figure 7.7: Scatter plots of downrange and cross-range fragment displacements as functions of fragment radius, for combined simulations. Both interacting and noninteracting cases are included. 

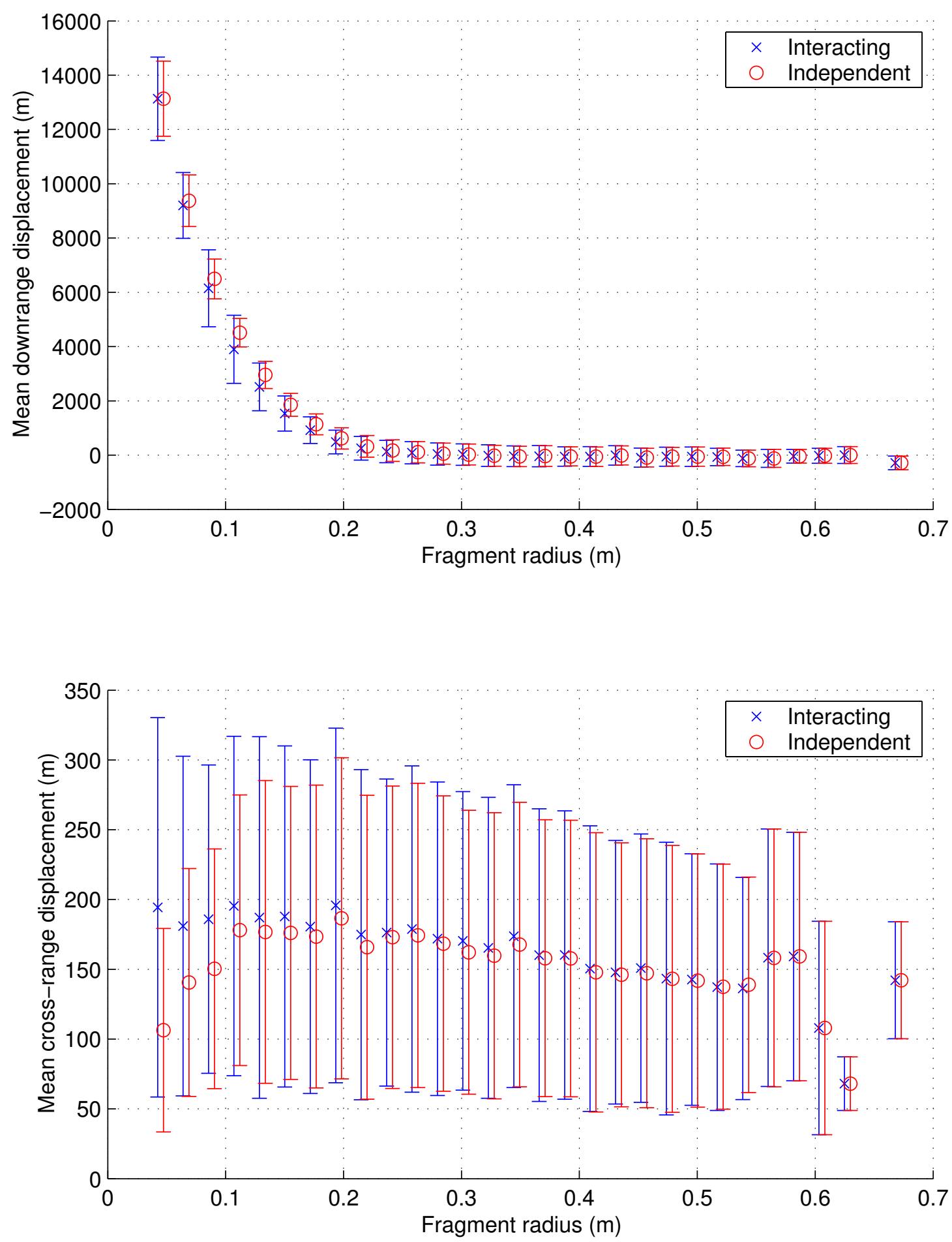

Figure 7.8: Mean downrange and cross-range displacements for fragments grouped by radius. These are the combined fragments from twelve simulations. 
For each fragment in each simulation, the final distances to all other fragments in that simulation were calculated and placed in ascending order. These distances were calculated separately for the interacting and non-interacting cases. In Figure 7.9, the mean values of these distances over all fragments and all simulations are plotted. To obtain the value corresponding to $n=4$, for example, for each fragment in a given simulation the distance to the fourth-closest neighboring fragment was chosen, and this distance was averaged over all fragments in all the simulations (note that this data should strictly be plotted discretely, but differences between the two cases can be seen more clearly when the plotting is continuous). We see that, except at the two extremes, i.e., for the closest and most distant fragments, the mean distances in the interacting cases are slightly less than those in the non-interacting cases. This indicates that aerodynamic interactions lead to slightly more compact overall crater fields. In Figures 7.7 and 7.8 we noted a tendency in the interacting simulations for smaller fragments to land further downrange, closer to the main field, but also at larger cross-range displacements. The effect seen in Figure 7.9 would result if this former tendency were dominating the latter.

Note, however, that for smaller values of $n$ (i.e., $n \lesssim 20$ ), there is very little difference between the interacting and non-interacting cases. If there was a tendency for increased secondary grouping in the interacting cases, we might expect to see a difference here. However, in this plot we have considered all possible pairs of fragments, whereas it is unlikely that all fragments would be affected by such a tendency. Thus, we now consider only the $50 \%$ of fragments in each simulation that have a closest neighbor, as it is reasonable to expect that these would be the fragments most likely to form part of a secondary group. In Figure 7.10, the mean distance to the $n$-th nearest fragment for only these fragments over all simulations is plotted. As may be seen, the mean values are typically smaller for the interacting cases than the non-interacting cases. 


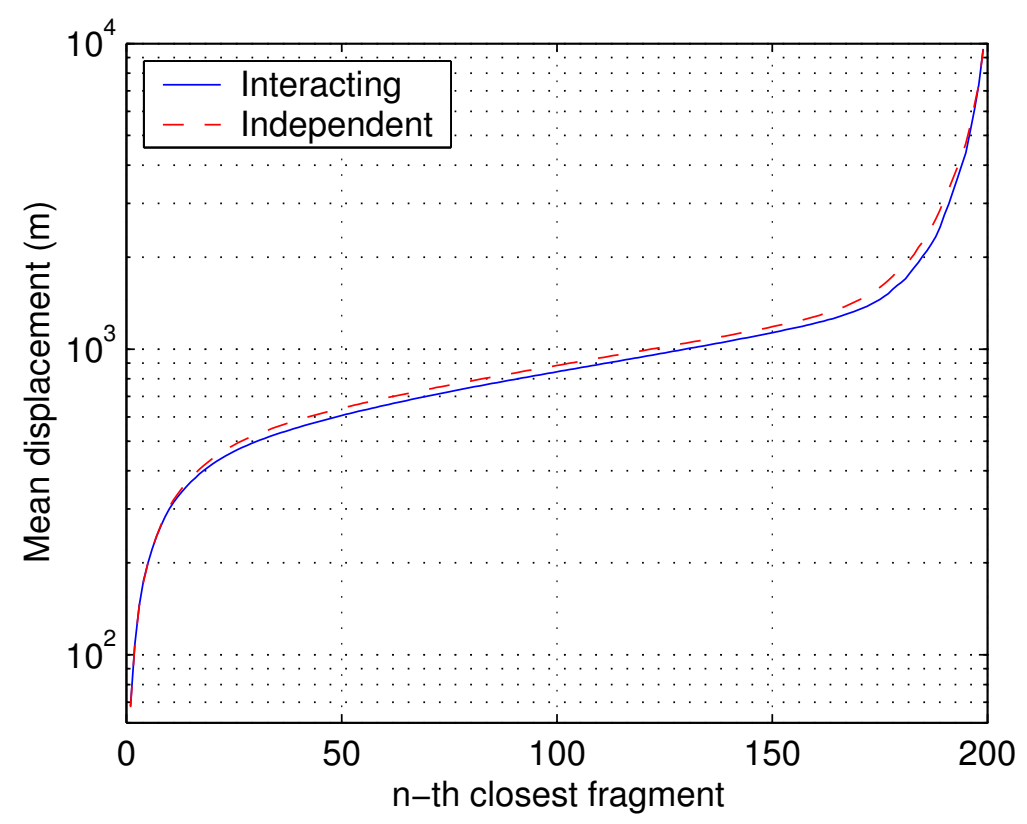

Figure 7.9: Plot of the mean distance from each fragment in a given simulation to the $n$-th closest fragment, averaged over all fragments in all simulations.

To determine whether the differences in mean values between the two cases is statistically significant, paired $t$ tests were performed on the sample groups for each value of $n$. The null hypothesis in each case was that there was no statistically significant difference between the two mean distances. The results from these tests for $n \leq 20$ are tabulated in Table 7.1. As may be seen, the significance level for rejection of the null hypothesis is better than $1 \%$ for up to $n=13$. This indicates that there is very strong evidence that the mean distances are not equal in the interacting and non-interacting cases for the thirteen closest fragments (and there is still strong evidence at the $5 \%$ level up to $n=20)$. We cannot conclude immediately that the aerodynamic interactions are leading to increased grouping, however, as the tendency for interacting simulations to lead to more compact crater fields, as noted previously, could also result in a similar effect. To determine whether this is the case, in Table 7.1 are also tabulated the results from tests that were identical except that the $90 \%$ of fragments with closest neighbors in each simulation were included. If the difference in means were a result of a tendency to more compact overall crater fields, we would 


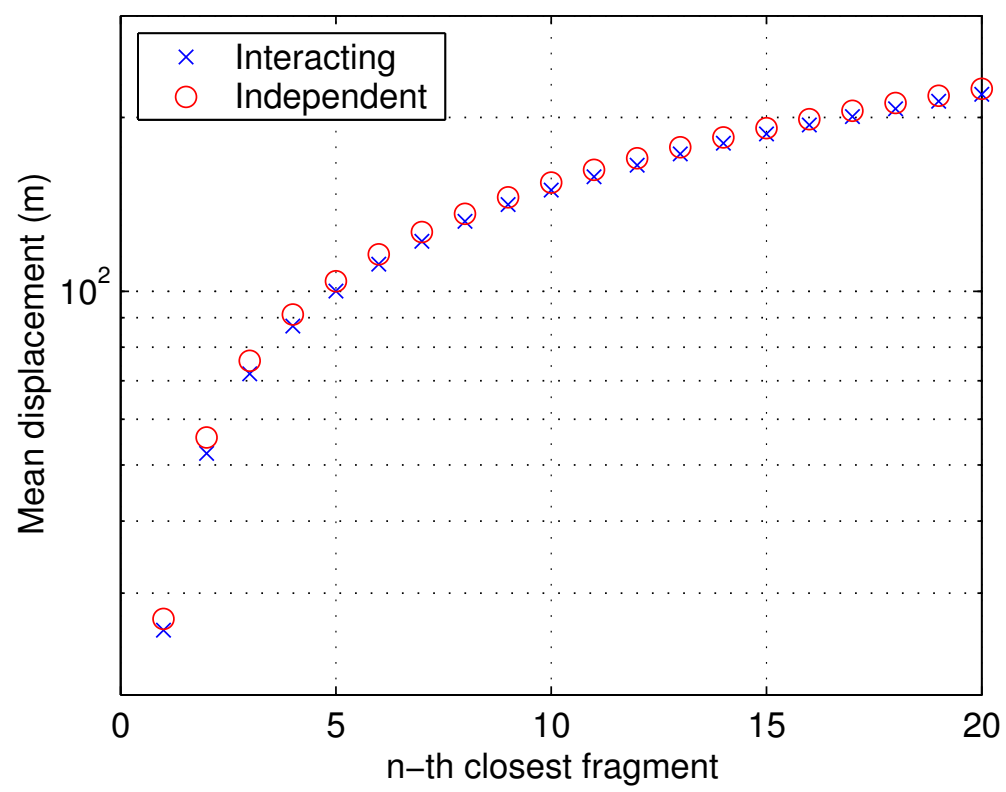

Figure 7.10: Plot of the mean distance from the $50 \%$ of fragments with nearest neighbors in a given simulation to the $n$-th closest fragment, averaged over all simulations.

expect the difference to be present for the larger group of fragments as well (the remaining $10 \%$ of fragments in each was excluded as these are likely to be outliers that have fallen short of the main field, and could thus skew the statistics). The evidence against the null hypothesis of equal means in these tests is much weaker - in fact, it is weaker than the $10 \%$ level for all but the sixth closest fragment for $n \leq 13$. This evidence actually becomes stronger for $n>13$ - this is probably a result of the trend to more compact fields in the interacting case. However, the combination of the two results for $n \leq 13$ gives strong statistical evidence that increased secondary grouping is present in those simulations in which aerodynamic interactions are included.

\subsubsection{Conclusions}

Having concluded that increased secondary grouping is produced by aerodynamic interactions, we wish to determine whether these groupings are consistent with those that would be produced by secondary fragmentation. To this end, we go back to the plots of crater locations in Figures 7.5 and 7.6. First, we may exclude groups such 


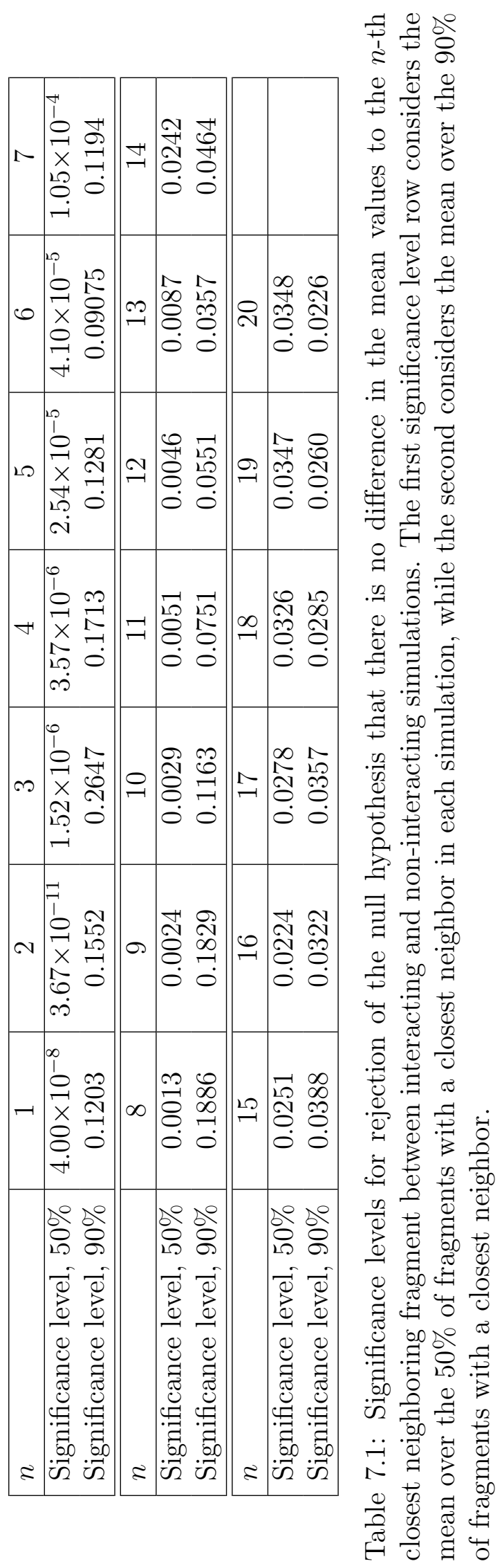


as that seen to the lower right of the origin in the top field of Figure 7.5, as this is centered on a large fragment that we would not expect to be present after secondary fragmentation. Those groups that involve a large number of smaller fragments, however, such as the uprange groups in the two lower fields, would not be excluded on this basis. In the case of secondary fragmentation, however, the resulting secondary crater fields will be generally of the same shape as the primary field, i.e., roughly elliptical with smaller fragments located near the uprange end. This shape is not generally observed in the secondary groupings in the fields of Figures 7.5 and 7.6, nor in the fields produced by other simulations in the sample set described above. This suggests that aerodynamic interactions and secondary fragmentation have similar but not identical effects on the secondary distribution of craters in the field. If a convincing, physically-based fragmentation model were available, simulations including the effects of secondary fragmentation would cast further light on this issue. As we lack such a model, however, we cannot draw truly definitive conclusions here. 


\section{Chapter 8}

\section{Conclusions}

In the current work we have sought to gain a general understanding of the dynamic effects at work in the hypersonic proximal bodies problem. In particular we have investigated the forces acting on a secondary body when some part of it is within the shocked region created by a primary body travelling at hypersonic speeds.

An analytical model has been developed based on the blast wave analogy for cases in which the secondary body is positioned entirely inside the primary shocked region. This allowed us to predict the force coefficients acting on the secondary body for simple body geometries in both two and three dimensions. The methodology predicted the nature of the lateral force coefficient to depend strongly on the relative size of the two bodies. For the case of two spheres it was found that, if the secondary body diameter was larger than one-sixth that of the primary body, the lateral force coefficient would be attractive throughout the shocked region. For smaller secondary bodies a repulsive lateral coefficient was possible. The results obtained using this analytical model have been compared with values obtained from numerical simulations using the AMROC software in both two and three dimensions, and these have generally shown good agreement provided an appropriate normalization is used for the lateral displacement.

To investigate the three-dimensional proximal body problem further, a series of experiments has been carried out in the T5 hypervelocity shock tunnel. This neces- 
sitated the development of a new force-measurement technique, and in a series of validation experiments, it was shown that this technique could successfully measure the drag on a sphere. In simulations of the proximal body problem, good agreement was obtained between measured forces and those predicted by perfect-gas numerical simulations. An error analysis was performed, and several areas in which further work could be warranted were identified.

The blast wave methodology was extended and used to simulate the entry of the binary asteroid system 2000 DP107 into the Earth's atmosphere. It was found that a very shallow entry angle was required for a system of this size for the aerodynamic interactions to become important. The methodology was also used to investigate the passage of a fragmented meteoroid through the Earth's atmosphere. In particular, the question was raised as to whether aerodynamic interactions, rather than secondary fragmentation, could be responsible for the secondary groupings observed in crater fields such as Sikhote-Alin. A statistical analysis provided strong evidence that aerodynamic interactions do lead to increased secondary crater grouping, but it was found that the shape of these groups did not exhibit the typically elliptical shape that we would expect secondary fragmentation to produce.

This thesis has been concerned exclusively with simple body geometries, i.e., cylinders in two dimensions and spheres in three dimensions. The most obvious way in which this work could be extended would be to consider more complex body geometries, particularly for the secondary body. One possibility would be to investigate bodies with intrinsic lift, in particular to determine whether the intrinsic lift contribution could be decoupled from that produced by the shocked flow. The analytical methodology that was developed in the current work relied on the secondary body possessing rotational symmetry, so an extension to more complex bodies would be difficult. The numerical software used, however, is in principle capable of modeling arbitrary geometries, so a computational approach would be feasible. Experimentally, the move 
to more complex geometries would require a refinement of the techniques developed here. The use of multiple accelerometers, located at appropriate positions, would allow multiple-component force-measurements as well as moment-measurement. Also, more sophisticated image-recognition algorithms would be required in order to enable the tracking of more complex body shapes in the high-speed visualisations. 


\section{Bibliography}

A. Ambrosio and A. Wortman. Stagnation point shock detachment distance for flow around spheres and cylinders. American Rocket Society Journal, 32:281, 1962. 75

N.A. Artem'eva and V.V. Shuvalov. Interaction of shock waves during the passage of a disrupted meteoroid through the atmosphere. Shock Waves, 5:359-367, 1996. 5

N.A. Artemieva and V.V. Shuvalov. Motion of a fragmented meteoroid through the planetary atmosphere. Journal of Geophysical Research, 106(E2):3297-3309, 2001. 5

R.B. Baldwin. The Measure of the Moon. University of Chicago Press, Chicago, 1963. 147

F.S. Billig. Shock-wave shapes around spherical- and cylindrical-nosed bodies. Journal of Spacecraft and Rockets, 4(6):822-823, 1967. 76

W.F. Bottke and H.J. Melosh. Binary asteroids and the formation of doublet craters. Icarus, 124:372-391, 1996. 113, 135

R. Deiterding. Construction and application of an AMR algorithm for distributed memory computers. In Adaptive Mesh Refinement - Theory and Applications, Proc. of the Chicago Workshop on Adaptive Mesh Refinement Methods, pages 361-372, 2003. 40 
W.E. Forsythe. Smithsonian Physical Tables (9th Revised Edition). Smithsonian Institute, Washington, 1954. 132

D.E. Gault. Impact cratering. In R. Greely and P. Schlitz, editors, A Primer in Lunar Geology. NASA Ames Research Center, 1974. 133

D.E. Grady. Local inertial effects in dynamic fragmentation. Journal of Applied Physics, 53(1):1210-1222, 1982. 149, 156

D.E. Grady and M.E. Kipp. Geometric statistics and dynamic fragmentation. Journal of Applied Physics, 58(3):1210-1222, 1985. 150

D.H. Hayes and R.R Probstein. Hypersonic Flow Theory. Academic Press, New York, 1966. 14

F. Heide. Meteorites. University of Chicago Press, Chicago, 1963. 160

H. Hornung, B. Sturtevant, J. Bélanger, S. Sanderson, and M. Brouillette. Performance of the new free-piston shock tunnel T5 at GALCIT. In Proceedings of the 18th International Symposium on Shock Waves, Sendai, Japan, July 1991. SpringerVerlag. 79

H.G. Hornung. Hypersonics. Class notes, 2002. 107

R. Joarder and J. Jagadeesh. A new free floating accelerometer balance system for force measurement in shock tunnels. Shock Waves, 13:409-412, 2004. 8

E.L. Krinov. Giant Meteorites, translated by J.S. Romankiewicz. Pergamon Press, New York, 1966. 158, 160

E.L. Krinov. Fragmentation of the sikhote-alin meteoritic body. Meteoritics, 9: 255-262, 1974. 148, 158 
L.D. Landau and E.M. Lifshitz. Fluid mechanics / by L.D. Landau and E.M. Lifshitz ; translated from the Russian by J.B. Sykes and W.H. Reid. Pergamon Press, Oxford, England; New York, 1989. 13, 28

P. Lemieux. The instability of shear layers produced by curved shocks. PhD thesis, California Institute of Technology, Pasadena, California, May 1999. 5

J.A. Lordi, R.E. Mates, and J.R. Moselle. Computer program for the numerical solution of nonequilibrium expansions of reacting gas mixtures. Technical Report NASA CR-472, NASA, 1966. 84, 188

J.L. Margot, M.C. Nolan, L.A.M. Benner, S.J. Ostro, R.F. Jurgens, J.D. Giorgini, M.A. Slade, and D.B. Campbell. Binary asteroids in the near-earth population. Science, 296:1445-1448, 2002. 113, 129

M.K. Mcintosh. A computer program fo the numerical calculation of equilibrium and perfect gas conditions in shock tunnels. Technical Report CPD 169, Australian Defense Scientific Service, 1969. 84, 187

D.J. Mee. Dynamic calibration of force balances for impulse hypersonic facilities. Shock Waves, 12:443-455, 2003. 7

D.J. Mee, W.J.T. Daniel, and J.M. Simmons. Three-component force balance for flows of millisecond duration. AIAA Journal, 34(3):590-595, 1996. 7

H.J. Melosh and J.A. Stansberry. Doublet craters and the tidal disruption of binary asteroids. Icarus, 94(171):171-179, 1991. 113

S.L. Meyer. Data analysis for scientists and engineers. John Wiley \& Sons, Inc., New York, 1975. 87 
K.W. Naumann, H. Ende, G. Mathieu, and A. George. Millisecond aerodynamic force measurement with side-jet model in the ISL shock tunnel. AIAA Journal, 31 (6):590-595, 1993. 8

Q.R. Passey and H.J. Melosh. Effects of atmospheric breakup on crater field formation. Icarus, 42:211-233, 1980. 5, 133, 135, 147, 155, 160, 163

J.J. Quirk. A computational facility (for cfd modeling). In VKI 29th CFD Lecture Series, Brussels, 1998. von Karman Institute for Fluid Dynamics. 1, 41

M.J. Robinson, D.J. Mee, C.Y. Tsai, and R.J. Bakos. Three-component force measurements on a large scramjet in a shock tunnel. Journal of Spacecraft and Rockets, 41(3):416-425, 2004. 7

N. Sahoo, D.R. Mahapatra, G. Jagadesh, S. Gopalakrishnan, and K.P.G. Reddy. An accelerometer balance system for measurement of aerodynamic force coefficients over blunt bodies in a hypersonic shock tunnel. Measurement Science and Technology, 14:260-272, 2003. 8

S.R. Sanderson and J.M. Simmons. Drag balance for hypervelocity impulse facilities. AIAA Journal, 12:2185-2191, 1991. 7

P.H. Schultz and S. Sugita. Penetrating and escaping the atmospheres of venus and earth. In Lunar and Planetary Science Conference XXV, pages 1215-1216, 1994. 5

L.I. Sedov. Similarity and dimensional methods in mechanics. Translation edited by Maurice Holt. Translation by Morris Friedman from the 4 th Russian ed. Academic Press, New York, 1959. 11

R.J. Stalker. Technical Report MT-44, National Research Council of Canada, 1961. 77 
V. Storkmann, H. Olivier, and H. Gronig. Force measurement in hypersonic impulse facilities. AIAA Journal, 36:342-348, 1998. 7

H. Tanno, K. Itoh, K. Saito, A. Abe, and K. Takayama. Shock wave interaction with a sphere in a shock tube. In International Symposium on Interdisciplinary Shock Wave Research, pages 483-497. Interdisciplinary Shock Wave Research Center, 2004. 8

G.I. Taylor. The formation of a blast wave by a very intense explosion. Proceedings of the Royal Society of London, A201(6):159-174, 1950. 10

P.A. Thompson. Compressible-fluid dynamics. Advanced Engineering Series, 1988. 92

M.J. Wright, G.V. Candler, and D. Bose. Data-parallel line relaxation method for the Navier-Stokes equations. AIAA Journal, 36(9):1603-1609, 1998. 107 


\section{Appendix A}

\section{Force Coefficients for Gaussian Pressure Distribution}

In this appendix we give the full expressions for the drag and lift coefficients derived in Chapter 2 for the Gaussian pressure distributions in both two and three dimensions.

In the two-dimensional case, the integrals in Equations 2.27 and 2.28 may be evaluated to give

$$
\begin{aligned}
C_{D}= & \frac{\sqrt{\pi}}{4}\left\{e^{-1 / 4}\left[\operatorname{erf}\left(\pi-\frac{\imath}{2}\right)+\operatorname{erf}\left(\pi+\frac{\imath}{2}\right)\right] p_{\text {stag }}^{\prime} \cos \delta\right. \\
& -\frac{1}{4 e} \frac{d_{2}}{d_{1}}[\operatorname{erf}(\pi-\imath)+\operatorname{erf}(\pi+\imath)] \frac{\partial p_{\text {stag }}^{\prime}}{\partial\left(r / d_{1}\right)} \sin 2 \delta \\
& \left.-\frac{1}{2} \frac{d_{2}}{d_{1}}\left(\operatorname{erf}(\pi)+\frac{1}{2 e}[\operatorname{erf}(\pi-\imath)+\operatorname{erf}(\pi+\imath)] \cos 2 \delta\right) \frac{\partial p_{\text {stag }}^{\prime}}{\partial\left(x / d_{1}\right)}\right\} \\
C_{L}= & \frac{\sqrt{\pi}}{4}\left\{e^{-1 / 4}\left[\operatorname{erf}\left(\pi-\frac{\imath}{2}\right)+\operatorname{erf}\left(\pi+\frac{\imath}{2}\right)\right] p_{\text {stag }}^{\prime} \sin \delta\right. \\
& -\frac{1}{4 e} \frac{d_{2}}{d_{1}}[\operatorname{erf}(\pi-\imath)+\operatorname{erf}(\pi+\imath)] \frac{\partial p_{\text {stag }}^{\prime}}{\partial\left(x / d_{1}\right)} \sin 2 \delta \\
& \left.-\frac{1}{2} \frac{d_{2}}{d_{1}}\left(\operatorname{erf}(\pi)-\frac{1}{2 e}[\operatorname{erf}(\pi-\imath)+\operatorname{erf}(\pi+\imath)] \cos 2 \delta\right) \frac{\partial p_{\text {stag }}^{\prime}}{\partial\left(r / d_{1}\right)}\right\} .
\end{aligned}
$$


In the three-dimensional case, an evaluation of the $\phi$ integrals in Equations 2.53 and 2.54 leads to

$$
\begin{aligned}
C_{D}= & 2 \cos \delta\left[p_{\text {stag }}^{\prime} \int_{0}^{\pi} \exp \left(-k \theta^{2}\right) \sin \theta \cos \theta d \theta\right. \\
& \left.+\frac{1}{2} \frac{d_{2}}{d_{1}}\left(\frac{\partial p_{\text {stag }}^{\prime}}{\partial\left(x / d_{1}\right)} \cos \delta-\frac{\partial p_{\text {stag }}^{\prime}}{\partial\left(r / d_{1}\right)} \sin \delta\right) \int_{0}^{\pi} \exp \left(-k \theta^{2}\right) \sin \theta \cos ^{2} \theta d \theta\right] \\
& +\frac{1}{2} \frac{d_{2}}{d_{1}}\left(\frac{\partial p_{\text {stag }}^{\prime}}{\partial\left(r / d_{1}\right)} \cos \delta-\frac{\partial p_{\text {stag }}^{\prime}}{\partial\left(x / d_{1}\right)} \sin \delta\right) \sin \delta \int_{0}^{\pi} \exp \left(-k \theta^{2}\right) \sin ^{3} \theta d \theta \\
C_{L}= & 2 \sin \delta\left[p_{\text {stag }}^{\prime} \int_{0}^{\pi} \exp \left(-k \theta^{2}\right) \sin \theta \cos \theta d \theta\right. \\
& \left.+\frac{1}{2} \frac{d_{2}}{d_{1}}\left(\frac{\partial p_{\text {stag }}^{\prime}}{\partial\left(x / d_{1}\right)} \cos \delta-\frac{\partial p_{\text {stag }}^{\prime}}{\partial\left(r / d_{1}\right)} \sin \delta\right) \int_{0}^{\pi} \exp \left(-k \theta^{2}\right) \sin \theta \cos ^{2} \theta d \theta\right] \\
& -\frac{1}{2} \frac{d_{2}}{d_{1}}\left(\frac{\partial p_{\text {stag }}^{\prime}}{\partial\left(r / d_{1}\right)} \cos \delta-\frac{\partial p_{\text {stag }}^{\prime}}{\partial\left(x / d_{1}\right)} \sin \delta\right) \cos \delta \int_{0}^{\pi} \exp \left(-k \theta^{2}\right) \sin ^{3} \theta d \theta .
\end{aligned}
$$

The $\theta$ integrals in Equations A.3 and A.4 may be evaluated to give the following unwieldy expressions:

$$
\begin{aligned}
& \int_{0}^{\pi} \exp \left(-k \theta^{2}\right) \sin \theta \cos \theta d \theta= \\
& \frac{1}{8 \imath e^{1 / k}} \sqrt{\frac{\pi}{k}}\left[\operatorname{erf}\left(\frac{k \pi-\imath}{\sqrt{k}}\right)+\operatorname{erfc}\left(\frac{k \pi+\imath}{\sqrt{k}}\right)+2 \imath \operatorname{erfi}\left(\frac{1}{\sqrt{k}}\right)-1\right], \\
& \int_{0}^{\pi} \exp \left(-k \theta^{2}\right) \sin \theta \cos ^{2} \theta d \theta= \\
& \quad \frac{1}{16 e^{9 / 4 k}} \sqrt{\frac{\pi}{k}}\left\{\imath \operatorname{erf}\left(\frac{2 k \pi+3 \imath}{2 \sqrt{k}}\right)-\imath \operatorname{erf}\left(\frac{2 k \pi-3 \imath}{2 \sqrt{k}}\right)+2 \operatorname{erfi}\left(\frac{3}{2 \sqrt{k}}\right)\right. \\
& \left.+e^{2 / k}\left[\imath \operatorname{erf}\left(\frac{2 k \pi+\imath}{2 \sqrt{k}}\right)-\imath \operatorname{erf}\left(\frac{2 k \pi-\imath}{2 \sqrt{k}}\right)+2 \operatorname{erfi}\left(\frac{1}{2 \sqrt{k}}\right)\right]\right\},
\end{aligned}
$$


and

$$
\begin{aligned}
\int_{0}^{\pi} & \exp \left(-k \theta^{2}\right) \sin ^{3} \theta d \theta= \\
& \frac{1}{16 e^{9 / 4 k}} \sqrt{\frac{\pi}{k}}\left\{3 e^{2 / k}\left[\imath \operatorname{erf}\left(\frac{2 k \pi+\imath}{2 \sqrt{k}}\right)-\imath \operatorname{erf}\left(\frac{2 k \pi-\imath}{2 \sqrt{k}}\right)+2 \operatorname{erfi}\left(\frac{1}{2 \sqrt{k}}\right)\right]\right. \\
& \left.+\imath\left[\operatorname{erf}\left(\frac{2 k \pi-3 \imath}{2 \sqrt{k}}\right)+\operatorname{erfc}\left(\frac{2 k \pi+3 \imath}{2 \sqrt{k}}\right)+2 \imath \operatorname{erfi}\left(\frac{3}{2 \sqrt{k}}\right)-1\right]\right\},
\end{aligned}
$$

where erfc is the complimentary error function, and erfi is the imaginary error function $\operatorname{erfi}(z)=\operatorname{erf}(\imath z) / \imath$.

For $k=1.2$, these expressions take values of $0.2469,0.1970$, and 0.1664 respectively. 


\section{Appendix B}

\section{T5 Run Conditions}

Listed here is the run information for all relevant shots in the T5. Included are the operating conditions, stagnation conditions, and freestream conditions at both the secondary and, where relevant, the primary sphere.

\begin{tabular}{cc|cccc|cc}
\hline $\begin{array}{c}\text { Shot } \\
\text { no. }\end{array}$ & $\begin{array}{c}\text { Test } \\
\text { gas }\end{array}$ & $\begin{array}{c}\mathrm{P}_{2 R} \\
(\mathrm{psi})\end{array}$ & $\begin{array}{c}\mathrm{P}_{C T} \\
(\mathrm{kPa})\end{array}$ & $\begin{array}{c}\% \mathrm{He} \\
(\mathrm{MPa})\end{array}$ & $\begin{array}{c}\mathrm{P}_{S T} \\
(\mathrm{MJ} / \mathrm{kg})\end{array}$ & $\mathrm{P}_{0}$ & $\mathrm{~h}_{0}$ \\
\hline 2290 & $\mathrm{CO}_{2}$ & 405 & 50 & 86 & 21 & 16.6 & 8.8 \\
2291 & $\mathrm{CO}_{2}$ & 405 & 50 & 86 & 21 & 16.9 & 9.1 \\
2292 & $\mathrm{CO}_{2}$ & 405 & 50 & 86 & 21 & 17.5 & 9.5 \\
2320 & $\mathrm{CO}_{2}$ & 405 & 50 & 86 & 21 & 19.7 & 9.0 \\
2321 & $\mathrm{CO}_{2}$ & 405 & 50 & 86 & 21 & 18.9 & 9.8 \\
2322 & $\mathrm{CO}_{2}$ & 405 & 50 & 86 & 21 & 20.0 & 9.6 \\
2324 & $\mathrm{~N}_{2}$ & 600 & 82.5 & 78 & 65 & 21.3 & 6.34 \\
2325 & $\mathrm{~N}_{2}$ & 450 & 62 & 90 & 37.2 & 17.0 & 10.7 \\
2326 & $\mathrm{CO}_{2}$ & 405 & 50 & 86 & 21 & 16.4 & 9.55 \\
2327 & $\mathrm{CO}_{2}$ & 405 & 50 & 86 & 21 & 16.8 & 9.58 \\
2328 & $\mathrm{CO}_{2}$ & 405 & 50 & 86 & 21 & 18.1 & 9.40 \\
2329 & $\mathrm{CO}_{2}$ & 405 & 50 & 86 & 21 & 17.4 & 9.44 \\
2330 & $\mathrm{CO}_{2}$ & 405 & 50 & 86 & 21 & 18.2 & 9.48 \\
\hline
\end{tabular}

Table B.1: Operating and stagnation conditions for all T5 shots relevant to this investigation. Stagnation enthalpies are calculated using ESTC (see Mcintosh (1969)). 


\begin{tabular}{c|ccccc|ccccc}
\hline \multirow{2}{*}{$\begin{array}{c}\text { Shot } \\
\text { no. }\end{array}$} & $\mathrm{P}_{\infty}$ & $\mathrm{T}_{\infty}$ & $\rho_{\infty}$ & $\mathrm{u}_{\infty}$ & $\mathrm{M}_{\infty}$ & $\mathrm{P}_{\infty}$ & $\mathrm{T}_{\infty}$ & $\rho_{\infty}$ & $\mathrm{u}_{\infty}$ & $\mathrm{M}_{\infty}$ \\
$(\mathrm{kPa})$ & $(\mathrm{K})$ & $\left(\mathrm{kg} / \mathrm{m}^{3}\right)$ & $(\mathrm{m} / \mathrm{s})$ & & $(\mathrm{kPa})$ & $(\mathrm{K})$ & $\left(\mathrm{kg} / \mathrm{m}^{3}\right)$ & $(\mathrm{m} / \mathrm{s})$ \\
\hline 2290 & - & - & - & - & - & 12.6 & 1888 & 0.0315 & 3044 & 4.47 \\
2291 & - & - & - & - & - & 12.8 & 1920 & 0.0312 & 3082 & 4.48 \\
2292 & - & - & - & - & - & 13.2 & 1973 & 0.0310 & 3148 & 4.48 \\
2320 & 15.7 & 1954 & 0.0381 & 3072 & 4.44 & 14.5 & 1932 & 0.0354 & 3084 & 4.48 \\
2321 & 15.0 & 2029 & 0.0340 & 3185 & 4.45 & 13.9 & 2008 & 0.0318 & 3195 & 4.49 \\
2322 & 16.0 & 2023 & 0.0367 & 3158 & 4.44 & 14.9 & 2004 & 0.0346 & 3168 & 4.48 \\
2324 & 7.43 & 776 & 0.0323 & 3324 & 5.93 & 6.80 & 758 & 0.0302 & 3330 & 6.01 \\
2325 & 7.22 & 1460 & 0.0166 & 4242 & 5.61 & 6.70 & 1434 & 0.0157 & 4250 & 5.67 \\
2326 & 14.3 & 2001 & 0.0331 & 3127 & 4.41 & 12.2 & 1958 & 0.0288 & 3149 & 4.49 \\
2327 & 14.4 & 2001 & 0.0331 & 3127 & 4.41 & 12.0 & 1953 & 0.0283 & 3152 & 4.50 \\
2328 & 16.0 & 2007 & 0.0372 & 3108 & 4.40 & 13.3 & 1959 & 0.0317 & 3133 & 4.49 \\
2329 & 15.3 & 2003 & 0.0355 & 3113 & 4.40 & 12.4 & 1947 & 0.0295 & 3142 & 4.51 \\
2330 & 16.0 & 2016 & 0.0369 & 3121 & 4.40 & 12.9 & 1960 & 0.0307 & 3150 & 4.51 \\
\hline
\end{tabular}

Table B.2: Freestream conditions at distances downstream corresponding to the leading points of the primary and secondary spheres. These and the values in the following table were calculated using NENZF (Lordi et al. (1966)).

\begin{tabular}{c|cccc|cc}
\hline Shot no. & $\mathrm{CO}_{2}$ & $\mathrm{CO}$ & $\mathrm{O}_{2}$ & $\mathrm{O}$ & $\mathrm{N}_{2}$ & $\mathrm{~N}$ \\
\hline 2290 & 0.69 & 0.20 & 0.10 & 0.01 & - & - \\
2291 & 0.67 & 0.22 & 0.10 & 0.01 & - & - \\
2292 & 0.64 & 0.24 & 0.11 & 0.01 & - & - \\
2320 & 0.69 & 0.20 & 0.10 & 0.01 & - & - \\
2321 & 0.62 & 0.25 & 0.12 & 0.01 & - & - \\
2322 & 0.64 & 0.24 & 0.11 & 0.01 & - & - \\
2324 & - & - & - & - & 1.000 & 0.000 \\
2325 & - & - & - & - & 0.996 & 0.004 \\
2326 & 0.63 & 0.24 & 0.12 & 0.01 & - & - \\
2327 & 0.63 & 0.24 & 0.12 & 0.01 & - & - \\
2328 & 0.65 & 0.23 & 0.11 & 0.01 & - & - \\
2329 & 0.65 & 0.23 & 0.11 & 0.01 & - & - \\
2329 & 0.65 & 0.23 & 0.11 & 0.01 & - & - \\
\hline
\end{tabular}

Table B.3: Freestream test gas concentrations in mole fractions. The downstream reference point is the secondary sphere, but changes in composition are small between the primary and the secondary sphere. In all $\mathrm{CO}_{2}$ shots the concentration of atomic carbon was negligible. 


\section{Appendix C}

\section{Calculation of the Drag Coefficient in the Impinging Case}

Here we will evaluate the drag coefficient in the general impinging case discussed in Section 6.3. The more difficult integrals in this section were evaluated using Mathematica. The flow geometry in the present situation is shown in Figure 6.5. The modified Newtonian pressure distribution will be assumed on the upper and lower regions, but the value of $p_{\text {stag }}^{\prime}$ will differ in each case, as explained in Section 6.3.

The primary shock is assumed to cut the sphere as a plane, and thus the contribution to the drag from the upper, singly-shocked region is

$$
\begin{aligned}
C_{D}^{u}= & \frac{1}{\pi}\left(p_{\text {stag }}^{\prime}-p_{\infty}^{\prime}\right) \int_{\alpha}^{\pi / 2} \int_{\arcsin \left(\frac{\sin \alpha}{\sin \theta}\right)}^{\pi-\arcsin \left(\frac{\sin \alpha}{\sin \theta}\right)} \cos ^{2} \theta \sin \theta(\sin \delta \sin \phi \sin \theta+\cos \delta \cos \theta) d \phi d \theta \\
& +\frac{1}{\pi} p_{\infty}^{\prime} \int_{\alpha}^{\pi-\alpha} \int_{\arcsin \left(\frac{\sin \alpha}{\sin \theta}\right)}^{\pi-\arcsin \left(\frac{\sin \alpha}{\sin \theta}\right)} \sin \theta(\sin \delta \sin \phi \sin \theta+\cos \delta \cos \theta) d \phi d \theta \\
= & \frac{1}{\pi}\left(p_{\text {stag }}^{\prime}-p_{\infty}^{\prime}\right)\left[\sin \delta \int_{\alpha}^{\pi / 2} \int_{\arcsin \left(\frac{\sin \alpha}{\sin \theta}\right)}^{\pi-\arcsin \left(\frac{\sin \alpha}{\sin \theta}\right)} \sin \phi \sin ^{2} \theta \cos ^{2} \theta d \phi d \theta\right. \\
& \left.+\cos \delta \int_{\alpha}^{\pi-\alpha} \int_{\arcsin \left(\frac{\sin \alpha}{\sin \phi}\right)}^{\pi / 2} \sin \theta \cos ^{3} \theta d \theta d \phi\right] \\
& +\frac{1}{\pi} p_{\infty}^{\prime}\left[\sin \delta \int_{\alpha}^{\pi-\alpha} \int_{\arcsin \left(\frac{\sin \alpha}{\sin \theta}\right)}^{\pi-\arcsin \left(\frac{\sin \alpha}{\sin \theta}\right)} \sin \phi \sin ^{2} \theta d \phi d \theta\right. \\
& \left.+\cos \delta \int_{\alpha}^{\pi-\alpha} \int_{\arcsin \left(\frac{\sin \alpha}{\sin \theta}\right)}^{\pi-\arcsin \left(\frac{\sin \alpha}{\sin \theta}\right)} \sin \theta \cos \theta d \theta d \phi\right] .
\end{aligned}
$$


Note the reversal of the order of integration in the second and fourth terms in C.1. This was necessary in order to enable these double integrals to be evaluated. We may evaluate the integrals in C.1 as follows:

$$
\begin{aligned}
& \int_{\alpha}^{\pi / 2} \int_{\arcsin \left(\frac{\sin \alpha}{\sin \theta}\right)}^{\pi-\arcsin \left(\frac{\sin \alpha}{\sin \theta}\right)} \sin \phi \sin ^{2} \theta \cos ^{2} \theta d \phi d \theta \\
& =2 \int_{\alpha}^{\pi / 2} \sin \theta \cos ^{2} \theta\left(\sin ^{2} \theta-\sin ^{2} \alpha\right)^{1 / 2} d \theta \\
& =\frac{\pi}{8} \cos ^{4} \alpha \\
& \int_{\alpha}^{\pi-\alpha} \int_{\arcsin \left(\frac{\sin \alpha}{\sin \phi}\right)}^{\pi / 2} \sin \theta \cos ^{3} \theta d \theta d \phi \\
& =\frac{1}{4} \int_{\alpha}^{\pi-\alpha}\left(1-\frac{\sin ^{2} \alpha}{\sin ^{2} \theta}\right)^{2} d \phi \\
& =\frac{1}{4}\left(\pi-2 \alpha-\frac{4}{3} \sin 2 \alpha-\frac{1}{6} \sin 4 \alpha\right) \\
& \int_{\alpha}^{\pi-\alpha} \int_{\arcsin \left(\frac{\sin \alpha}{\sin \theta}\right)}^{\pi-\arcsin \left(\frac{\sin \alpha}{\sin \theta}\right)} \sin \phi \sin ^{2} \theta d \phi d \theta \\
& =2 \int_{\alpha}^{\pi-\alpha} \sin \theta\left(\sin ^{2} \theta-\sin ^{2} \alpha\right)^{1 / 2} d \theta \\
& =\pi \cos ^{2} \alpha \\
& \int_{\alpha}^{\pi-\alpha} \int_{\arcsin \left(\frac{\sin \alpha}{\sin \theta}\right)}^{\pi-\arcsin \left(\frac{\sin \alpha}{\sin \theta}\right)} \sin \theta \cos \theta d \theta d \phi=0
\end{aligned}
$$

Our expression for $C_{D}^{u}$ then becomes

$$
\begin{aligned}
C_{D}^{u}= & \left(p_{\text {stag }}^{\prime}-p_{\infty}^{\prime}\right)\left[\frac{1}{8} \sin \delta \cos ^{4} \alpha+\frac{\cos \delta}{4 \pi}\left(\pi-2 \alpha-\frac{4}{3} \sin 2 \alpha-\frac{1}{6} \sin 4 \alpha\right)\right] \\
& +p_{\infty}^{\prime} \sin \delta \cos ^{2} \alpha
\end{aligned}
$$

On the lower doubly-shocked side, we wish to include the $x$-derivative terms, and 
our expression for this contribution to the drag coefficient is given by

$$
\begin{aligned}
C_{D}^{l}= & \frac{1}{\pi}\left[\int_{0}^{2 \pi} \int_{0}^{\pi / 2}-\int_{\alpha}^{\pi-\alpha} \int_{\arcsin \left(\frac{\sin \alpha}{\sin \phi}\right)}^{\pi / 2}\right. \\
& \left(\left(p_{\text {stag }}^{\prime}-p_{1}^{\prime}\right)-\frac{1}{2} \frac{d_{2}}{d_{1}} \frac{\partial\left(p_{\text {stag }}^{\prime}-p_{1}^{\prime}\right)}{\partial\left(x / d_{1}\right)} \hat{x}\right) \sin \theta \cos ^{2} \theta \hat{x} d \theta d \phi \\
& \left.+\int_{0}^{2 \pi} \int_{0}^{\pi}-\int_{\alpha}^{\pi-\alpha} \int_{\arcsin \left(\frac{\sin \alpha}{\sin \phi}\right)}^{\pi-\arcsin \left(\frac{\sin \alpha}{\sin \phi}\right)}\left(p_{1}^{\prime}-\frac{1}{2} \frac{d_{2}}{d_{1}} \frac{\partial p_{1}^{\prime}}{\partial\left(x / d_{1}\right)} \hat{x}\right) \sin \theta \hat{x} d \theta d \phi\right]
\end{aligned}
$$

where $\hat{x}=\sin \delta \sin \phi \sin \theta+\cos \delta \cos \theta$. After some algebra, we obtain

$$
\begin{aligned}
& C_{D}^{l}=\frac{1}{\pi}\left(p_{\text {stag }}^{\prime}-p_{1}^{\prime}\right)\left[\sin \delta \int_{0}^{\pi / 2} \int_{0}^{2 \pi}-\int_{\alpha}^{\pi / 2} \int_{\arcsin \left(\frac{\sin \alpha}{\sin \theta}\right)}^{\pi-\arcsin \left(\frac{\sin \alpha}{\sin \theta}\right)} \sin \phi \sin ^{2} \theta \cos ^{2} \theta d \phi d \theta\right. \\
& \left.+\cos \delta \int_{0}^{2 \pi} \int_{0}^{\pi / 2}-\int_{\alpha}^{\pi-\alpha} \int_{\arcsin \left(\frac{\sin \alpha}{\sin \phi}\right)}^{\pi / 2} \sin \theta \cos ^{3} \theta d \theta d \phi\right] \\
& -\frac{1}{2 \pi} \frac{d_{2}}{d_{1}} \frac{\partial\left(p_{\text {stag }}^{\prime}-p_{1}^{\prime}\right)}{\partial\left(x / d_{1}\right)}\left[\sin ^{2} \delta \int_{0}^{2 \pi} \int_{0}^{\pi / 2}-\int_{\alpha}^{\pi-\alpha} \int_{\arcsin \left(\frac{\sin \alpha}{\sin \phi}\right)}^{\pi / 2} \sin ^{3} \theta \cos ^{2} \theta \sin ^{2} \phi d \theta d \phi\right. \\
& +2 \sin \delta \cos \delta \int_{0}^{2 \pi} \int_{0}^{\pi / 2}-\int_{\alpha}^{\pi-\alpha} \int_{\arcsin \left(\frac{\sin \alpha}{\sin \phi}\right)}^{\pi / 2} \sin ^{2} \theta \cos ^{3} \theta \sin \phi d \theta d \phi \\
& \left.+\cos ^{2} \delta \int_{0}^{2 \pi} \int_{0}^{\pi / 2}-\int_{\alpha}^{\pi-\alpha} \int_{\arcsin \left(\frac{\sin \alpha}{\sin \phi}\right)}^{\pi / 2} \sin \theta \cos ^{4} \theta d \theta d \phi\right] \\
& +\frac{1}{\pi} p_{1}^{\prime}\left[\sin \delta \int_{0}^{\pi} \int_{0}^{2 \pi}-\int_{\alpha}^{\pi-\alpha} \int_{\arcsin \left(\frac{\sin \alpha}{\sin \theta}\right)}^{\pi-\arcsin \left(\frac{\sin \alpha}{\sin \theta}\right)} \sin \phi \sin ^{2} \theta d \phi d \theta\right. \\
& \left.+\cos \delta \int_{0}^{2 \pi} \int_{0}^{\pi}-\int_{\alpha}^{\pi-\alpha} \int_{\arcsin \left(\frac{\sin \alpha}{\sin \phi}\right)}^{\pi-\arcsin \left(\frac{\sin \alpha}{\sin \phi}\right)} \sin \theta \cos \theta d \theta d \phi\right] \\
& -\frac{1}{2 \pi} \frac{d_{2}}{d_{1}} \frac{\partial p_{1}^{\prime}}{\partial\left(x / d_{1}\right)}\left[\sin ^{2} \delta \int_{0}^{2 \pi} \int_{0}^{\pi}-\int_{\alpha}^{\pi-\alpha} \int_{\arcsin \left(\frac{\sin \alpha}{\sin \phi}\right)}^{\pi-\arcsin \left(\frac{\sin \alpha}{\sin \phi}\right)} \sin ^{3} \theta \sin ^{2} \phi d \theta d \phi\right. \\
& +2 \sin \delta \cos \delta \int_{0}^{2 \pi} \int_{0}^{\pi}-\int_{\alpha}^{\pi-\alpha} \int_{\arcsin \left(\frac{\sin \alpha}{\sin \phi}\right)}^{\pi-\arcsin \left(\frac{\sin \alpha}{\sin \phi}\right)} \sin ^{2} \theta \cos \theta \sin \phi d \theta d \phi \\
& \left.+\cos ^{2} \delta \int_{0}^{2 \pi} \int_{0}^{\pi}-\int_{\alpha}^{\pi-\alpha} \int_{\arcsin \left(\frac{\sin \alpha}{\sin \phi}\right)}^{\pi-\arcsin \left(\frac{\sin \alpha}{\sin \phi}\right)} \sin \theta \cos ^{2} \theta d \theta d \phi\right] .
\end{aligned}
$$

Again the order of some of the integrals has been reversed in order to allow the relevant double integral to be evaluated. The integrals involving $\alpha$ may be evaluated 
as follows:

$$
\begin{aligned}
& \int_{\alpha}^{\pi-\alpha} \int_{\arcsin \left(\frac{\sin \alpha}{\sin \phi}\right)}^{\pi / 2} \sin ^{3} \theta \cos ^{2} \theta \sin ^{2} \phi d \theta d \phi \\
& =\frac{1}{15} \int_{\alpha}^{\pi-\alpha}\left(1-\frac{\sin ^{2} \alpha}{\sin ^{2} \phi}\right)^{3 / 2}\left(2 \sin ^{2} \phi+3 \sin ^{2} \alpha\right) d \phi \\
& =\frac{\pi}{480}(32-30 \sin \alpha+5 \sin 3 \alpha+3 \sin 5 \alpha) \\
& \int_{\alpha}^{\pi-\alpha} \int_{\arcsin \left(\frac{\sin \alpha}{\sin \phi}\right)}^{\pi / 2} \sin ^{2} \theta \cos ^{3} \theta \sin \phi d \theta d \phi \\
& =\frac{1}{15} \int_{\alpha}^{\pi-\alpha}\left(2-5 \frac{\sin ^{3} \alpha}{\sin ^{3} \phi}+3 \frac{\sin ^{5} \alpha}{\sin ^{5} \phi}\right) \sin \phi d \phi \\
& =\frac{4}{15} \cos ^{5} \alpha \int_{\alpha}^{\pi-\alpha} \int_{\arcsin \left(\frac{\sin \alpha}{\sin \phi}\right)}^{\pi / 2} \sin \theta \cos ^{4} \theta d \theta d \phi \\
& =\frac{1}{5} \int_{\alpha}^{\pi-\alpha}\left(1-\frac{\sin ^{2} \alpha}{\sin ^{2} \phi}\right)^{5 / 2} d \phi \\
& =\frac{\pi}{640}(128-150 \sin \alpha-25 \sin 3 \alpha-3 \sin 5 \alpha) \\
& \int_{\alpha}^{\pi-\alpha} \int_{\arcsin \left(\frac{\sin \alpha}{\sin \phi}\right)}^{\pi-\arcsin \left(\frac{\sin \alpha}{\sin \phi}\right)} \sin ^{3} \theta \sin ^{2} \phi d \theta d \phi \\
& =\frac{2}{3} \int_{\alpha}^{\pi-\alpha}\left(1-\frac{\sin ^{2} \alpha}{\sin ^{2} \phi}\right)^{1 / 2}\left(2 \sin ^{2} \phi+\sin ^{2} \alpha\right) d \phi \\
& =\frac{2 \pi}{3}\left(1-\sin ^{3} \alpha\right) \\
& \int_{\alpha}^{\pi-\alpha} \int_{\arcsin \left(\frac{\sin \alpha}{\sin \phi}\right)}^{\pi-\arcsin \left(\frac{\sin \alpha}{\sin \phi}\right)} \sin ^{2} \theta \cos \theta \sin \phi d \theta d \phi=0 \\
& \int_{\alpha}^{\pi-\alpha} \int_{\arcsin \left(\frac{\sin \alpha}{\sin \phi}\right)}^{\pi-\arcsin \left(\frac{\sin \alpha}{\sin \phi}\right)} \sin \theta \cos ^{2} \theta d \theta d \phi \\
& =\frac{2}{3} \int_{\alpha}^{\pi-\alpha}\left(1-\frac{\sin ^{2} \alpha}{\sin ^{2} \phi}\right)^{3 / 2} d \phi \\
& =\frac{\pi}{12}(8-9 \sin \alpha-\sin 3 \alpha) \text {. }
\end{aligned}
$$


Also:

$$
\begin{aligned}
\int_{0}^{2 \pi} \int_{0}^{\pi / 2} \sin ^{2} \theta \cos ^{2} \theta \sin \phi d \theta d \phi & =0 \\
\int_{0}^{2 \pi} \int_{0}^{\pi / 2} \sin \theta \cos ^{3} \theta d \theta d \phi & =0 \\
\int_{0}^{2 \pi} \int_{0}^{\pi / 2} \sin ^{3} \theta \cos ^{2} \theta \sin ^{2} \phi d \theta d \phi & =\frac{2 \pi}{15} \\
\int_{0}^{2 \pi} \int_{0}^{\pi / 2} \sin ^{2} \theta \cos ^{3} \theta \sin \phi d \theta d \phi & =0 \\
\int_{0}^{2 \pi} \int_{0}^{\pi / 2} \sin \theta \cos ^{4} \theta d \theta d \phi & =\frac{2 \pi}{5} \\
\int_{0}^{2 \pi} \int_{0}^{\pi} \sin { }^{2} \theta \sin \phi d \theta d \phi & =0 \\
\int_{0}^{2 \pi} \int_{0}^{\pi} \sin \theta \cos \theta d \theta d \phi & =0 \\
\int_{0}^{2 \pi} \int_{0}^{\pi} \sin { }^{3} \theta \sin { }^{2} \phi d \theta d \phi & =\frac{4 \pi}{3} \\
\int_{0}^{2 \pi} \sin ^{2} \theta \cos \theta \sin \phi d \theta d \phi & =0 \\
\int_{0}^{2 \pi} \int_{0}^{\pi} \sin \theta \cos { }^{2} \theta d \theta d \phi & =\frac{4 \pi}{3} .
\end{aligned}
$$

Combining these with the integrals evaluated for the upper region, we may write the lower contribution as:

$$
\begin{aligned}
C_{D}^{l}= & \frac{1}{\pi}\left(p_{\text {stag }}^{\prime}-p_{1}^{\prime}\right)\left[\cos \delta\left(\frac{\pi}{4}+\frac{\alpha}{2}+\frac{\sin 2 \alpha}{3}+\frac{\sin 4 \alpha}{24}\right)-\frac{\pi}{8} \sin \delta \cos ^{4} \alpha\right] \\
& -p_{1}^{\prime} \sin \delta \cos ^{2} \alpha-\frac{1}{2} \frac{d_{2}}{d_{1}} \frac{\partial\left(p_{\text {stag }}^{\prime}-p_{1}^{\prime}\right)}{\partial\left(x / d_{1}\right)}\left[\sin ^{2} \delta\left(\frac{1}{15}+\frac{\sin \alpha}{16}-\frac{\sin 3 \alpha}{96}-\frac{\sin 5 \alpha}{160}\right)\right. \\
& \left.-\frac{8}{15 \pi} \sin \delta \cos \delta \cos ^{5} \alpha+\cos ^{2} \delta\left(\frac{1}{5}+\frac{15}{64} \sin \alpha+\frac{5}{128} \sin 3 \alpha+\frac{3}{640} \sin 5 \alpha\right)\right] \\
& -\frac{1}{2} \frac{d_{2}}{d_{1}} \frac{\partial p_{1}^{\prime}}{\partial\left(x / d_{1}\right)}\left[\frac{2}{3} \sin ^{2} \delta\left(1+\sin ^{3} \alpha\right)+\cos ^{2} \delta\left(\frac{2}{3}+\frac{3}{4} \sin \alpha-\frac{\sin 3 \alpha}{12}\right)\right]
\end{aligned}
$$

